\title{
A Compact, Discrete CsI(Tl) Scintillator/Si Photodiode Gamma Camera for Breast Cancer Imaging
}

by

\section{Gregory Jerome Gruber}

B.S. (University of Wisconsin at Madison) 1994

M.S. (University of California at Berkeley) 1996

A dissertation submitted in partial satisfaction of the

requirements for the degree of

Joint Doctor of Philosophy

with University of California at San Francisco

in

Bioengineering

in the

GRADUATE DIVISION

of the

\section{UNIVERSITY OF CALIFORNIA AT BERKELEY}

Committee in charge:

Professor Stephen Derenzo, Chair

Professor Bruce Hasegawa

Professor David Attwood

Fall 2000 
The dissertation of Gregory Jerome Gruber is approved:

\begin{tabular}{lr}
\hline Chair & Date \\
\hline & Date \\
\hline University of California at Berkeley & \\
\hline
\end{tabular}

Fall 2000 


\title{
A Compact, Discrete CsI(Tl) Scintillator/Si Photodiode Gamma Camera for Breast Cancer Imaging
}

\author{
Copyright 2000
}

by

Gregory Jerome Gruber 


\begin{abstract}
A Compact, Discrete CsI(Tl) Scintillator/Si Photodiode

Gamma Camera for Breast Cancer Imaging

by

Gregory Jerome Gruber

Joint Doctor of Philosophy with University of California at San Francisco in

Bioengineering

University of California at Berkeley

Professor Stephen Derenzo, Chair
\end{abstract}

Recent clinical evaluations of scintimammography (radionuclide breast imaging) are promising and suggest that this modality may prove a valuable complement to X-ray mammography and traditional breast cancer detection and diagnosis techniques. Scintimammography, however, typically has difficulty revealing tumors that are less than $1 \mathrm{~cm}$ in diameter, are located in the medial part of the breast, or are located in the axillary nodes. These shortcomings may in part be due to the use of large, conventional Anger cameras not optimized for breast imaging. In this thesis I present compact single photon camera technology designed specifically for scintimammography which strives to alleviate some of these limitations by allowing better and closer access to sites of possible breast tumors. Specific applications are outlined.

The design is modular, thus a camera of the desired size and geometry can be constructed from an array (or arrays) of individual modules and a parallel hole lead collimator for directional information. Each module consists of: (1) an array of 64 discrete, optically-isolated $\mathrm{CsI}(\mathrm{Tl})$ scintillator crystals $3 \times 3 \times 5 \mathrm{~mm}^{3}$ in size, (2) an array of 64 low-noise Si PIN photodiodes matched 1-to-1 to the scintillator 
crystals, (3) an application-specific integrated circuit (ASIC) that amplifies the 64 photodiode signals and selects the signal with the largest amplitude, and (4) connectors and hardware for interfacing the module with a motherboard, thereby allowing straightforward computer control of all individual modules within a camera.

The two truly innovative components in this design are the silicon photodiodes and the ASIC readout. The photodiodes have been developed specifically for low room temperature leakage current (they average $26 \mathrm{pA}$ per $3 \times 3 \mathrm{~mm}^{2}$ pixel at $50 \mathrm{~V}$ bias) and high quantum efficiency (>80\% for $540 \mathrm{~nm}$ light). This is critical to achieving low electronic noise and high signal, respectively, thereby yielding good energy resolution and allowing the photodiodes to replace the bulky, expensive, lownoise photomultiplier tubes (PMTs) employed in conventional Anger cameras. My specific contributions include developing design specifications, non-destructive dicing techniques, and assembly and packaging procedures that integrate the delicate arrays into imaging modules. I further made detailed studies of the photodiode leakage current, quantum efficiency, signal levels, and noise performance.

ASIC readout is key to compact design because of the high pixel density. The 64-channel ASIC is fabricated using a standard 3.3 V 0.5 $\mu \mathrm{m}$ CMOS process and is challenging to implement because it is a mixed analog-digital IC with a high-gain front end and strict requirements for minimal electronic noise $\left(\sim 180 \mathrm{e}^{-}\right.$rms at $8 \mu \mathrm{s}$ peaking time with a photodiode load). The ASIC reduces 64 analog inputs to a single analog "winner" (the one with the largest amplitude) and a 6-bit digital address denoting the winning channel (identified within $150 \mathrm{~ns}$ ), contributing to compactness. The specific advances I have made include developing specifications, characterizing and debugging prototype front end designs, characterizing and debugging a complete prototype readout system employing multiple ICs, physically and electrically integrating the ASIC into the compact imaging modules, and characterizing the ASIC performance in these modules.

I developed Monte Carlo simulation code implementing a 3-D phantom patient, Compton scattering, and photoelectric absorption to study and optimize 
collimator geometry and detector pixel size for breast cancer imaging. Other relevant factors, including energy resolution and the level of tracer uptake by the tumor, were also explored. The results demonstrate the importance of a high sensitivity collimator $(>16,000$ counts $/ \mathrm{mCi} / \mathrm{sec})$, the diminishing improvement in image quality achieved by making pixels much smaller than $3 \times 3 \mathrm{~mm}^{2}$, the small advantages of using square collimator holes instead of hexagonal ones, and the unimportance of improving energy resolution much beyond $15 \%$ full-width at half-max (fwhm). The simulated planar images suggest tumors smaller than $1 \mathrm{~cm}$ may be observable but highlight the inherent difficulties, especially when tumor diameter is $\leq 0.75 \mathrm{~cm}$.

Finally, I explore the assembly and performance of 64-pixel detector modules and present a detailed design for a 16-module, $9.6 \times 9.6 \mathrm{~cm}^{2}$ camera appropriate for scintimammography applications. The total depth of the modules depends on the collimator selected, but is quite compact at less than $4 \mathrm{~cm}$. My design for the modules and related camera are based upon both the Monte Carlo simulation studies and on my characterization of prototype 12-pixel modules constructed from preliminary versions of the critical hardware. A prototype 64-pixel module demonstrates average energy resolution of $20.0 \pm 2.6 \%$ fwhm for $122 \mathrm{keV}$ gammas from ${ }^{57} \mathrm{Co}$, or an estimated $17.4 \pm 2.3 \%$ fwhm for $140 \mathrm{keV}$ gammas from ${ }^{99 m} \mathrm{Tc}$. A flood source exciting the detector array without a collimator produces a reasonably uniform image with a standard deviation of $2.7 \%$ of the average count rate. Finally, a point source (in air or with scattering media) exciting a module equipped with a collimator produces images with the anticipated spatial resolution. More thorough testing and development of the 64-pixel module and especially the complete 16-module camera will certainly be needed before advancing to clinical trials, but the prospects for this new technology are very promising.

Professor Stephen Derenzo

Dissertation Committee Chair 


\section{To Jill}

It may have been a long road, but it was worth travelling 


\section{Acknowledgements}

I could not have completed this work without the help of many people, and I am deeply indebted to all of them. I would like to start by acknowledging the tremendous contributions Bill Moses has made to my graduate school experience. Although he never served as my advisor in an official capacity, Bill guided my Ph.D. work day in and day out and has been a mentor to me in everything from how to debug an integrated circuit to how to remain happy while working through the frustrations inherent in research. Bill has been an exemplary role model both as a researcher and as a person, and it is hard to imagine how I could possibly have completed this degree without his supervision, insight, and friendship. I also owe a great deal of thanks to my advisor, Steve Derenzo, for guiding my graduate research for both my M.S. and Ph.D. projects, as well as for encouraging me to pursue nuclear medicine and medical imaging. Throughout the course of my work he has always proven an invaluable source of expertise and support. The leader of our Center for Functional Imaging family, Tom Budinger, is also very deserving of my thanks, for not only has he has shown a great deal of faith and confidence in me, but he has constantly pushed both my project and my research efforts forward. I also thank the Center for Functional Imaging staff as a whole, for certainly everyone who works there has contributed to my wonderful experience as a graduate student researcher.

Bruce Hasegawa not only served on both my qualifying exam committee (as chair, no less) and my thesis committee, he also spearheaded several classes I took and was a constant figure of support and guidance at professional conferences. I still recall visiting with Bruce when I was first considering the joint UC Berkeley-UC San Francisco bioengineering program years ago, and he has never steered me wrong. David Attwood was also part of both my qualifying exam committee and my thesis committee, in addition to which he also served as second reader for my Master's thesis. I offer him my sincere thanks for his careful analysis of all my graduate work. I also wish to thank Gary Caputo, Dan Vigneron, and Marian Diamond for 
serving on my qualifying exam committee and for all the time they spent discussing my project with me both before and after the examination. Likewise I thank Nola Hylton for her numerous insights into the challenges of imaging breast cancer.

Marzio Pedrali-Noy, Emanuele Mandelli, Brad Krieger, Garrit Meddeler, and Eric Beuville deserve thanks for their tireless work in developing the custom integrated circuits that were critical to the success of my project. Learning about integrated circuits with them was often difficult and frustrating for all of us, but it was nonetheless incredibly fruitful. I am also greatly appreciative to Steve Holland and Nadine Wang for all the time and effort they devoted to designing, testing, and fabricating the advanced silicon photodiode arrays upon which much of my research depended, and to Craig Tindall for his assistance with some of the final photodiode processing steps. The advances made with these photodiodes made believers out of skeptics. And how could I have ever have built anything with these components without the help of George Zizka? He wirebonded countless assemblies, then had to wirebond them all over again whenever something failed.

Jenny Huber and Matt Ho were invaluable in the lab itself, for Jenny was always there to help me out (often offering cookies, too!) and Matt complained only slightly about all the times he had to design and build some piece of apparatus or another for me. Patrick Virador was also a common fixture around the lab, always willing to answer my programming questions. Together Jenny, Matt, and Patrick provided the friendship that made my work fun rather than just an obstacle. WoonSeng Choong proved a great addition to the lab toward the end of my project, and I thank him for carrying on a lot of work where I left off.

Eric Gawiser, Geoff and Susan Pilling, Andrew and Lisa Harris, Kenneth Wong and Andrea Kramer, Karen Calhoun, Julia Heinzerling, Tony Moy, and Roger Rabehl have long been good friends, and I thank them for everything they have done to make my life outside of work fun and enjoyable. Sometimes that is more important than anything else.

Most of all, I am indebted to my family members for their constant and 
unfaltering support, and especially to my parents for the guidance and encouragement they have provided throughout my life. I may not have followed the exact path they would have laid out for me, but very early on they let me forge my own way and have always stood lovingly beside me. Special thanks to my brother Garry for being a good friend and for reminding me how to approach life from perspectives other than my own, and to my Aunt Jeanne and my grandmothers Dawnine and Verona for all the love they have shown me over the years. It saddens me deeply that Verona was not able to see me complete this journey, for she surely had a role in my success. Finally, I want to thank my fiancée Jill, who has endured countless trips to the lab for one reason or another and has patiently listened to my many complaints about everything that has ever gone wrong during the course of this work. Her smile and laughter always made everything better, and her support, friendship, and love helped me maintain my faith in myself. I cannot thank her enough.

I am extremely grateful to the Fannie and John Hertz Foundation for the fellowship support that made this research possible. My work was also supported in part by a University of California at Berkeley Graduate Fellowship, in part by the Director, Office of Science, Office of Biological and Environmental Research, Medical Science Division of the U.S. Department of Energy under contract No. DE-AC0376SF00098, in part by the Laboratory Technology Research Division SC-32, (formerly called ER LTT), Office of Science, U.S. Department of Energy under a CRADA (Cooperative Research and Development Agreement) between Lawrence Berkeley National Laboratory and Capintec, Inc., Ramsey, NJ under U.S. DOE Contract DE-AC03-76SF00098, in part by the National Institutes of Health, National Cancer Institute under grant No. R01-CA67911, and in part by the National Institutes of Health, National Heart, Lung, and Blood Institute under grant No. P01-HL25840, and in part by the Breast Cancer Research Program of the University of California Grant No. 1RB-0068. 


\section{Contents}

List of Figures $\quad$ xi

List of Tables $\quad$ xiv

1 Introduction $\quad 1$

1.1 The Problem of Breast Cancer . . . . . . . . . . . . . . . 1

1.2 Single Photon Imaging . . . . . . . . . . . . . . . . . . 3

1.2.1 Basic Mechanisms . . . . . . . . . . . . 3

1.2 .2 The Collimator . . . . . . . . . . . . . . 7

1.2 .3 Statistical Noise . . . . . . . . . . . . . . . . . 10

1.2.4 Attenuation . . . . . . . . . . . . . . . . . 11

1.2.5 Scatter and Energy Resolution . . . . . . . . . . . . . . 12

1.3 Prospects for the Proposed Gamma Camera . . . . . . . . . . . . . . 13

1.3.1 Advantages . . . . . . . . . . . . . . . . 15

1.3.2 Specific Applications . . . . . . . . . . . . . . 15

1.4 Research Overview . . . . . . . . . . . . . . . . 17

2 Breast Cancer Morphology, Diagnosis, and Treatment 19

2.1 Pathology of Breast Cancer . . . . . . . . . . . . . . . . . 19

2.1.1 The Normal Breast . . . . . . . . . . . . . . . . . 20

2.1 .2 Carcinoma in Situ . . . . . . . . . . . . . . . . . . 23

2.1.3 Invasive Carcinoma . . . . . . . . . . . . . . . . . 24

2.1 .4 Cysts . . . . . . . . . . . . . . . . 25

2.1.5 Other Benign Disorders . . . . . . . . . . . . . 25

2.2 Detection and Diagnosis of Breast Cancer . . . . . . . . . . . . . 27

2.2.1 Physical Exam . . . . . . . . . . . . . . . . 27

2.2.2 X-ray Mammography and Screening . . . . . . . . . . . . 27

2.2.3 Strengths and Limitations of X-ray Mammography . . . . . . . 31 
2.2 .4 Biopsy . . . . . . . . . . . . . . . . . . . . 32

2.2.5 Staging . . . . . . . . . . . . . . 32

2.3 Treatment of Breast Cancer . . . . . . . . . . . . . . . . 33

2.3.1 Surgical Therapy . . . . . . . . . . . . . . . 34

2.3.2 Axillary Node Dissection . . . . . . . . . . . . . . 35

2.3 .3 Radiation Therapy . . . . . . . . . . . . . . . 36

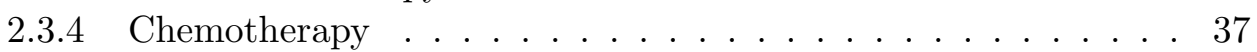

2.3.5 Hormone Therapy . . . . . . . . . . . . . . . 38

2.4 Other Imaging Modalities . . . . . . . . . . . . . . . . . . . 38

2.4.1 Scintimammography . . . . . . . . . . . . . . . 38

2.4.2 Positron Emission Mammography . . . . . . . . . . . . . . . . . . 42

2.4 .3 Ultrasound . . . . . . . . . . . . . . . . . . . . 43

2.4.4 Contrast-Enhanced MRI . . . . . . . . . . . . . . . . . 44

2.4.5 Summary of the Roles of Different Imaging Modalities . . . . . 45

3 Fundamentals of Single Photon Gamma Cameras 48

3.1 Anger Scintillation Cameras . . . . . . . . . . . . . . . . 48

3.1.1 Basic Principles . . . . . . . . . . . . . . . . . . . 49

3.1.2 NEMA Performance Characteristics . . . . . . . . . . . 56

3.2 Discrete Scintillator/Photodiode Cameras . . . . . . . . . . . . . . 61

3.2.1 Motivation for Compact Designs . . . . . . . . . . . . 61

3.2.2 CsI(Tl) Scintillator Coupled to Si PIN Photodiodes . . . . . . 62

3.2.3 NEMA Performance Characteristics . . . . . . . . . . . . 67

3.3 Solid-State CdZnTe Cameras . . . . . . . . . . . . . . . . . 68

3.4 Position Sensitive Photomultiplier Tube Cameras . . . . . . . . . . . . 70

4 Prototype CsI(Tl)/Si PIN Photodiode Detectors $\quad 72$

4.1 Electronic Readout and Pulse Shaping . . . . . . . . . . . . . . . 74

4.1.1 Basic Pulse Counting Systems . . . . . . . . . . . . . . 75

4.1 .2 Electronic Noise . . . . . . . . . . . . . . . . . 78

4.2 Prototype Detector System _ . . . . . . . . . . . . . . 83

4.2 .1 Module Design . . . . . . . . . . . . . . . . . 83

4.2.2 Prototype Low-noise Photodiodes . . . . . . . . . . . . . 85

4.2 .3 Prototype Readout ICs . . . . . . . . . . . . . . . . . . 86

4.2.4 Readout and Computer Acquisition . . . . . . . . . . . . . 88

4.2.5 Collimator Design . . . . . . . . . . . . . . 88

4.3 Detector Imaging Characteristics . . . . . . . . . . . . . . . . 92

4.3.1 Background Spectrum . . . . . . . . . . . . . . . 92

4.3.2 Energy Resolution . . . . . . . . . . . . . . . . 93 
4.3.3 Intrinsic Spatial Resolution . . . . . . . . . . . . . . . 95

4.3.4 System Spatial Resolution with a High Resolution Collimator . 96

4.3.5 System Spatial Resolution with a High Sensitivity Collimator . 102

4.4 Summary and Analysis . . . . . . . . . . . . . . . . . . . . . 104

5 Monte Carlo Simulations of Breast Tumor Imaging Characteristics 106

5.1 Simulation Methods . . . . . . . . . . . . . . . . . . 107

5.1.1 Verification of Simulation Code . . . . . . . . . . . . . . . 109

5.1 .2 Background Activity and Imaging Time . . . . . . . . . . . . 114

5.1.3 Generation of Background Events . . . . . . . . . . . . 115

5.1.4 The Rose Equation and Tumor Detectability . . . . . . . . . 116

5.1.5 Characterizing Tumor Images . . . . . . . . . . . . . . . . . 117

5.1.6 Expected Tumor Event Rate . . . . . . . . . . . . . . . . . . 119

5.2 Simulation Results . . . . . . . . . . . . . . . . . . . . 120

5.2.1 Typical Tumor Images and Background Patterns . . . . . . . . . 120

5.2.2 Collimator Sensitivity and Hole Shape . . . . . . . . . . . . 121

5.2 .3 Pixel Size . . . . . . . . . . . . . . . . . 126

5.2.4 Tumor-to-Background Tissue Uptake Ratio . . . . . . . . . . . 127

5.2.5 Detector Energy Resolution . . . . . . . . . . . . . . . . 129

5.3 Summary and Analysis . . . . . . . . . . . . . . . . 130

6 Custom Integrated Circuit Readout 134

6.1 Basic PETRIC Design . . . . . . . . . . . . . . . . . . . . . 134

6.2 Analog Front End . . . . . . . . . . . . . . . . . 137

6.2.1 Charge-Sensitive Preamplifier . . . . . . . . . . . . . . 139

6.2 .2 Shaper Amplifier . . . . . . . . . . . . . . . . . . . . 141

6.2.3 Prototype Performance . . . . . . . . . . . . . . . . 143

6.3 "Winner Take All" Mixed Analog/Digital Back End . . . . . . . . . . 147

6.3.1 Circuit Design . . . . . . . . . . . . . . . . . 148

6.3 .2 Prototype Performance . . . . . . . . . . . . 150

6.4 Complete PETRIC Chip . . . . . . . . . . . . . . . . . . . . . . 152

6.4.1 Design Changes Suggested by Prototype ICs . . . . . . . . . 154

6.4.2 Characterization of the PETRIC . . . . . . . . . . . 158

7 Complete 64-Pixel Imaging Modules $\quad \mathbf{1 6 4}$

7.1 Gamma Camera Design Parameters . . . . . . . . . . . . . . . 167

7.2 64-Pixel Low-noise Photodiode Arrays . . . . . . . . . . . . . . . 170

7.3 64-Pixel Module Design . . . . . . . . . . . . . . . . . . . . . 173

7.3.1 Printed Circuit Boards . . . . . . . . . . . . . . . . 174

7.3.2 Assembly Procedures . . . . . . . . . . . . . . . . 176 
7.3.3 Interface and Control . . . . . . . . . . . . . . . 178

7.4 Complete 16-Module Camera Design . . . . . . . . . . . . . . . . . 179

7.4.1 Camera Hierarchy and Motherboard Design . . . . . . . . . . 179

7.4 .2 Collimator Design . . . . . . . . . . . . . . 186

7.5 Single 64-Pixel Module Performance . . . . . . . . . . . . . . . . 188

7.5.1 Intrinsic Energy Resolution . . . . . . . . . . . . . . . 191

7.5.2 Uniformity of Response to a Flood Source . . . . . . . . . . . . 191

7.5 .3 Point Source Images . . . . . . . . . . . . . . . . . . 193

7.5.4 Module Performance Summary . . . . . . . . . . . . . 196

8 Conclusion $\quad 198$

8.1 Summary of Results . . . . . . . . . . . . . . . . . . . . 199

8.2 Future Work . . . . . . . . . . . . . . . . . . . 202

$\begin{array}{ll}\text { A Publications } & 206\end{array}$

$\begin{array}{ll}\text { Bibliography } & 208\end{array}$ 


\section{List of Figures}

1.1 Basic single photon imaging . . . . . . . . . . . . . . . . 4

1.2 Localization of radionuclide distribution with a collimator . . . . . 8

1.3 Photopeak and energy resolution for a monochromatic source . . . . . 14

1.4 Basic components of a compact, discrete gamma camera . . . . . . . . 14

2.1 Structure of the normal female breast . . . . . . . . . . . . . 21

2.2 X-ray mammography . . . . . . . . . . . . . . . . . . . . . . 29

2.3 Scintimammography basics . . . . . . . . . . . . . . . . . . 39

2.4 Chemical structure of ${ }^{99 m} \mathrm{Tc}$-sestamibi . . . . . . . . . . . . . . . . 41

2.5 Positron emission mammography $(\mathrm{PEM})$ camera . . . . . . . . . . . . . 44

2.6 Breast cancer detection and therapy decision tree . . . . . . . . . . . 47

3.1 Basic components of the Anger scintillation camera . . . . . . . . . . 51

3.2 Array of PMTs in an Anger scintillation camera . . . . . . . . . . . 53

3.3 Anger scintillation camera applied to scintimammography . . . . . . . 55

3.4 Anger camera dead space . . . . . . . . . . . . . . . 56

3.5 Dead time limits on camera count rate . . . . . . . . . . . . . . 60

3.6 Compact gamma camera applied to scintimammography . . . . . . . . 63

3.7 Scintillator crystal coupled to a photodiode . . . . . . . . . . . . 65

4.1 Basic components in a counting system . . . . . . . . . . . . 75

4.2 Ideal charge-sensitive preamp . . . . . . . . . . . . . . 76

4.3 Shaper using CR differentiation and RC integration . . . . . . . . . . 78

4.4 Unit step and delta noise sources . . . . . . . . . . . . . . . . . . 80

4.5 Charge-sensitive preamp with noise sources . . . . . . . . . . . . . . 81

4.6 Electronic noise versus shaper peaking time . . . . . . . . . . . . 84

4.7 Prototype discrete scintillation camera module with $3 \times 4$ pixels . . . 84

4.8 ASIC readout and computer acquisition for a prototype module . . . . 89 
4.9 Background spectrum for prototype module . . . . . . . . . . . . 92

4.10 Energy resolution for prototype module . . . . . . . . . . . . . . . . 94

4.11 Intrinsic spatial resolution for prototype module . . . . . . . . . . 97

4.12 System spatial resolution for prototype module . . . . . . . . . . . . . 97

4.13 Average spatial resolution with high resolution collimators . . . . . . . 98

4.14 Average spatial resolution with high sensitivity collimators . . . . . 103

5.1 Monte Carlo 3-D phantom patient . . . . . . . . . . . . . . . . 109

5.2 Compton scattering angles . . . . . . . . . . . . . . . . 111

5.3 Typical Monte Carlo tumor images . . . . . . . . . . . . . . . . . . 122

5.4 Typical Monte Carlo background pattern . . . . . . . . . . . . . 123

5.5 Mismatch between hexagonal collimator holes and square pixels . . . . 123

5.6 Dependence of tumor fwhm on choice of collimator . . . . . . . . . . 125

5.7 Dependence of tumor S/N on choice of collimator . . . . . . . . . . 125

5.8 Tumor size dominates tumor fwhm at short imaging distances . . . . . 127

5.9 Dependence of tumor fwhm on tumor diameter and pixel size . . . . . 128

5.10 Dependence of tumor $\mathrm{S} / \mathrm{N}$ on tumor diameter and pixel size . . . . . 128

5.11 Dependence of tumor $\mathrm{S} / \mathrm{N}$ on $\mathrm{T} / \mathrm{B}$ uptake ratio . . . . . . . . . . 130

5.12 Dependence of tumor fwhm on energy resolution . . . . . . . . . . 131

5.13 Dependence of tumor $\mathrm{S} / \mathrm{N}$ on energy resolution . . . . . . . . . . . 131

6.1 PETRIC block diagram . . . . . . . . . . . . . . . . . . . . . 138

6.2 Preamplifier circuit diagram . . . . . . . . . . . . . . . . . . . . 140

6.3 Shaper amplifier circuit diagram . . . . . . . . . . . . . . . . . . . 142

6.4 Equivalent noise charge versus shaper peaking time . . . . . . . . . . 146

6.5 Preamplifier reset time versus input load current . . . . . . . . . . . 148

6.6 Simplified WTA circuit diagram . . . . . . . . . . . . . . 150

6.7 Timing as the WTA output address switches . . . . . . . . . . . . 151

6.8 Overhead view of PETRIC layout . . . . . . . . . . . . . 153

6.9 PETRIC front end waveforms demonstrating gain . . . . . . . . 159

6.10 PETRIC front end waveforms with a range of peaking times . . . . . . 160

6.11 PETRIC electronic noise with capacitive loads . . . . . . . . . . . 161

6.12 PETRIC WTA performance . . . . . . . . . . . . . . . . 162

7.1 System level diagram for the 16 -module camera . . . . . . . . . . . 166

7.264 -Pixel low-noise photodiode array . . . . . . . . . . . . . 171

7.3 Histogram of photodiode leakage currents . . . . . . . . . . . . . 172

7.4 A complete 64-pixel module . . . . . . . . . . . . . . . . . . . . . . . . 174

7.5 Key components used in the 64 -pixel modules . . . . . . . . . . . . . 175

7.6 Conceptual design of a 16-module compact gamma camera . . . . . 180 
7.7 Peak detect circuitry on the 16-module motherboard . . . . . . . . . 184

7.8 Timing diagram for data acquisition signals in the 16-module camera . 185

7.9 Block diagram of the 16 -module motherboard . . . . . . . . . . . . 187

7.10 Intrinsic energy resolution of a 64 -pixel module . . . . . . . . . . . . . 192

7.11 Flood source response of a 64 -pixel module . . . . . . . . . . . . . . . 193

7.12 Point sources imaged by a 64 -pixel module . . . . . . . . . . . . 195 
xiv

\section{List of Tables}

3.1 Comparison of $\mathrm{NaI}(\mathrm{Tl})$ and $\mathrm{CsI}(\mathrm{Tl}) \ldots \ldots \ldots$. . . . . . . . . 64

5.1 Probability that an identified feature is a false alarm . . . . . . . . . 117

5.2 Expected number of detected tumor events . . . . . . . . . . . . 120

6.1 Summary of PETRIC characteristics . . . . . . . . . . . . . . 139

7.1 Three collimator designs for the 16 -module camera . . . . . . . . . . 189 


\section{Chapter 1}

\section{Introduction}

The stumbling way in which even the ablest of the scientists in every generation have had to fight through thickets of erroneous observations, misleading generalizations, inadequate formulations, and unconscious prejudice is rarely appreciated by those who obtain their scientific knowledge from textbooks.

—James B. Conant, Science and Common Sense, 1951

\subsection{The Problem of Breast Cancer}

Among women in the United States breast cancer is the second leading cause of cancer-related death, trailing only lung cancer. Every year approximately 180,000 new cases of breast cancer are reported and about 44,000 related deaths occur $[1,2]$. Further, the incidence of breast cancer has increased in recent decades $[3,4]$ and it is currently estimated that about one out of every eight women in the United States will at some point be diagnosed with the disease $[1,5]$. While various studies have demonstrated that mass screening with X-ray mammography leads to early detection and can thereby greatly improve prognosis and survival rate $[6,7,8]$, the mortality rate still remains disturbingly high - about $30 \%$ of those women who do develop the disease will die because of it $[1,9]$. 
Clearly millions of women in the United States alone are at risk for breast cancer, resulting in large medical expenditures and an unfortunately high level of patient suffering and morbidity. Some of these problems result directly from the limitations of current screening and diagnosis procedures. For example, every year some 700,000 mammography-positive women undergo biopsy (which is often uncomfortable, stressful, and expensive in time and money for the patient, can increase the patient's radiation exposure, and is time consuming for the physician) to determine whether a suspicious structure is malignant. However, only about $25 \%$ of the biopsied structures prove to be cancerous, leading many breast cancer experts to conclude that better diagnostic imaging techniques are needed. Factors like this coupled with the pervasive anxiety that surrounds potential victims and their families have generated a massive public awareness of breast cancer, its clinical management, and relevant medical research. Recent years have seen extensive media coverage of all facets of the disorder and a substantial increase in funding for research designed to better understand, detect, and treat breast tumors. Such research has ranged from studies of genetic predisposition to the disease (e.g., the BRCA1 and BRCA2 genes) to the development of breast-conserving lumpectomy surgeries effective in treating malignant lesions to the implementation of new medical imaging methods that aid in early detection and diagnosis.

Without question the most important breast cancer imaging modality is planar X-ray mammography, as its low cost (less than $\$ 100$ per exam), high spatial resolution $(\sim 100 \mu \mathrm{m})$, and very high sensitivity for the detection of breast lesions make it an excellent tool for screening the general population. However, X-ray mammography demonstrates several key limitations that motivate the development of other imaging methods that can play a complementary role in breast cancer detection. Among these other modalities is scintimammography, or the use of both a radionuclide tracer that accumulates preferentially in tumor tissue and a single photon gamma camera that generates an image of the resulting radionuclide distribution. Work in the field of scintimammography includes research into radionuclide 
and tumor biochemistry, the development of improved radionuclide tracers, clinical trials to refine imaging protocols and diagnostic decision-making, and the development of advanced imaging instrumentation designed specifically for this application. This last research and development area is the focus of this thesis.

\subsection{Single Photon Imaging}

\subsubsection{Basic Mechanisms}

The basic concept behind single photon imaging is the use of a radiationdetecting camera to visualize the distribution of a radionuclide tracer that has been administered to the patient, typically by injection. This radiopharmaceutical is chosen for its biochemical properties and ideally accumulates only in a specific type of tissue. A radioactive isotope is chemically included in the tracer molecule, thus as the tracer accumulates in the target tissue the radioactive isotope decays and emits a gamma ray with a particular characteristic energy. In general single photon imaging uses gamma emitters in the $80-500 \mathrm{keV}$ range because at these energies the gamma rays are sufficiently penetrating to emerge from the patient even if generated deep within the body, yet they are not so penetrating that they cannot be stopped by a reasonable thickness of lead (which is key to both collimator and shielding design). The most commonly used radioactive isotope in the field of single photon imaging is ${ }^{99 m} \mathrm{Tc}$, which has a half life of about 6 hours and emits a $140 \mathrm{keV}$ gamma ray.

Upon the decay of the radioactive isotope, the emitted gamma ray traverses the body - possibly scattering along the way - and if not absorbed by body tissue, exits the patient. At this point the single photon gamma camera potentially detects the radiation event and, if certain conditions are met, adds that information to the image data that are being collected. A collimator is employed in front of the actual radiation detection hardware in order to provide directional information on the trajectory of detected photons and their likely region of origin. This is accom- 


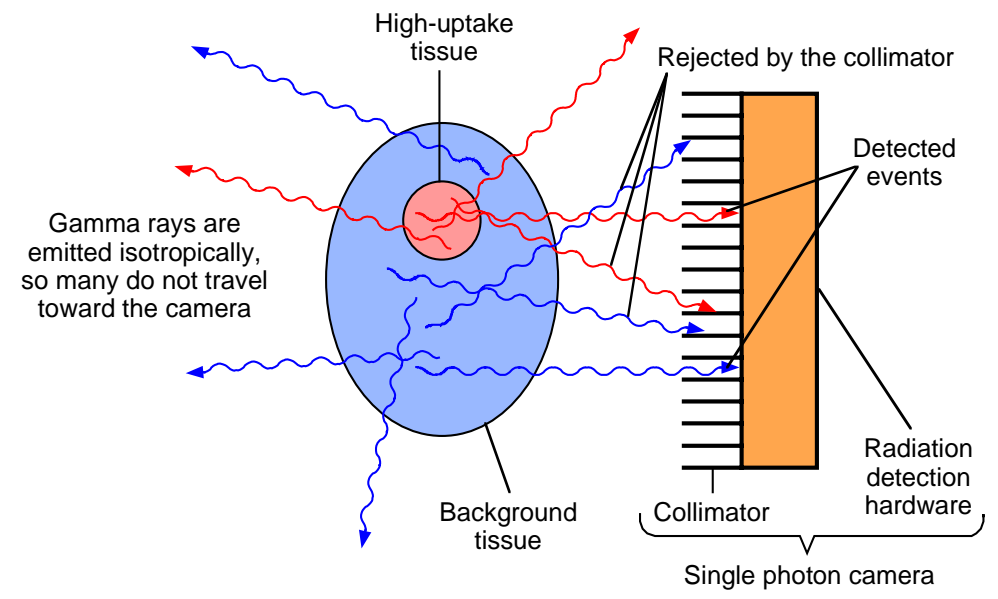

Figure 1.1: The basics of single photon imaging. A radionuclide tracer administered to the patient accumulates preferentially in a specific tissue type (e.g., tumors), and gamma rays emitted from the tracer are detected by a single photon camera equipped with a collimator. Red gammas illustrate desirable photons from high-uptake tissue, while blue gammas represent background photons.

plished through simple geometrical effects and is known as absorptive collimationonly photons travelling in a narrow range of trajectories are likely to pass through the collimator without being absorbed, thereby preventing undesirable photons from reaching the detector system and contaminating the image data. Such a collimated single photon detector system is depicted in Figure 1.1.

The radiation detection hardware itself operates by converting the energy of incident gamma rays into electrical signals that can be processed by downstream electronics. The conventional means of doing this is to employ scintillator material that converts individual gamma rays into many visible light photons, then directs those photons to a photodetector that converts their energy into an electric signal. Some camera technology instead employs solid-state detectors that absorb the gamma rays directly and convert their energy into an electrical signal without the intermediate conversion to visible light photons. 
Single photon imaging can be divided into two categories, namely planar imaging and single photon emission computed tomography (SPECT). Planar imaging is the simpler and less expensive of the two, and involves taking a two-dimensional projection image of the patient by placing the camera in a single location and acquiring data. The resulting image is a projection through the patient in that the camera "looks through" the patient's body in one particular direction. This naturally creates a superposition of some tissues within the image, much like viewing multiple transparencies stacked on top of each other. Frequently this superposition is undesirable, which motivates the use of SPECT. In SPECT multiple planar images are taken of the patient from a variety of angles, and the data from this set of two-dimensional images are used to reconstruct a three-dimensional image tomographically. This tomographic reconstruction can be done either analytically by means of filtered back projection (FBP) or with iterative techniques that progressively improve an estimate for the three-dimensional distribution of the radionuclide tracer. Analytic FBP techniques are relatively simple and computationally swift, but in most cases iterative techniques are more accurate (but are more computationally intensive). The threedimensional data set produced by tomographic reconstruction has the advantages of spatial localization in all three dimensions, superior contrast between high-uptake tissue and background tissue, and the potential to allow quantitation studies. Simple planar imaging is performed far more often, however, and is obviously the preferred choice in cases where rotating the gamma camera around the target tissue (as is required for tomographic reconstruction) is challenging.

Single photon imaging is a useful diagnostic and research tool for a number of reasons. Perhaps most important is the nature of the information it provides. Because single photon imaging visualizes the distribution of a radionuclide tracer within a patient, its primary function is to study biochemical behavior and reveal the patient's functional physiology. This is in stark contrast to X-ray imaging, for instance, which instead reveals structural physiology. These two imaging modalities are thus very different in nature and are used to provide very different image information. 
Another strength of single photon imaging is the high contrast that can be achieved between high-uptake tissue and background tissue as a result of the impressive tissue specificity demonstrated by some radionuclide tracers. Single photon imaging is also very sensitive to small amounts of tracer material, which is key to both to not harming the patient and to not disturbing the tissue that is under observation. Scintimammography, for instance, is typically performed after an injection of $20 \mathrm{mCi}$ of ${ }^{99 m}$ Tc-sestamibi (Miraluma, Dupont Pharmaceutical Company, North Billerica, MA) followed by about $10 \mathrm{cc}$ of saline to clear the tracer from the vessel [10]. This also, however, indicates one of the significant drawbacks of this imaging modalitynot only is it necessary to inject the patient with a pharmaceutical, but because the tracer is radioactive the patient is exposed to a noteworthy internal radiation dose. Exposure levels are, of course, kept low enough to be entirely justified by the value of the information provided by the imaging study, but they are certainly worthy of consideration.

Finally, single photon imaging is but one of two techniques that fall under the umbrella of nuclear medicine imaging. The second is positron emission tomography $(\mathrm{PET})$, wherein the radionuclide tracer is a positron emitter. The resulting positron-electron annihilation produces two $511 \mathrm{keV}$ gamma rays that travel in opposite directions, forming a nearly straight line. As a result, PET detector systems use electronic collimation wherein only coincidence events (two events that are detected within an extremely short time of each other) are accepted, and each such pair of events forms a line of response along which the positron annihilation must have occurred. The lack of a physical collimator is a major advantage of PET-no events are lost due to absorption within the collimator and PET therefore enjoys much greater sensitivity than does single photon imaging. PET also has access to a different and sometimes more biologically interesting set of radionuclide tracers since isotopes that emit positrons differ from those that emit single photons. Single photon imaging instrumentation, however, tends to be significantly less expensive than that for PET, making it more readily available in the hospital setting. Further, single 
photon emitters are inexpensive and simple to produce in comparison to positron emitters.

\subsubsection{The Collimator}

The performance of single photon cameras of virtually every variety is heavily dependent on the collimator and its geometry. By its nature the collimator tends to be the limiting factor for both system spatial resolution and sensitivity. For instance, while the intrinsic spatial resolution of a traditional Anger gamma camera is 3-4 mm full width at half maximum (fwhm) and even less for some newer technologies, the spatial resolution of a standard collimator at an imaging distance of $50 \mathrm{~mm}$ is about $5.1 \mathrm{~mm}$ fwhm for a low-energy, high-resolution (LEHR) design, and about $5.6 \mathrm{~mm}$ fwhm for a low-energy, all-purpose (LEAP) design [11]. Further, because the system response of the camera is essentially that of the collimator convolved with that of the detector hardware, the collimator spatial resolution and the intrinsic spatial resolution add in quadrature to yield the system spatial resolution:

$$
R_{\text {system }} \approx \sqrt{\left(R_{\text {collimator }}\right)^{2}+\left(R_{\text {intrinsic }}\right)^{2}}
$$

where $R$ indicates the fwhm spatial resolution of the relevant stage. This squaring

effect naturally leads to the dominance of the collimator spatial resolution even when it is only slightly larger than the intrinsic resolution. Sensitivity is also dominated by the collimator: whereas most single photon detector systems have a detection efficiency above $90 \%$ (that is, less than $20 \%$ of the gammas can pass through the detector hardware unobserved), it is not uncommon for collimators to reject all but $0.1-0.01 \%$ of incoming gamma rays before they ever reach the detection hardware. Careful collimator design is thus critical because it is the weakest link for both of these key performance metrics.

Selecting a collimator geometry is largely a compromise between spatial resolution and detection efficiency. Figure 1.2 depicts the basic method by which a traditional parallel hole collimator localizes the tracer activity, providing spatial 


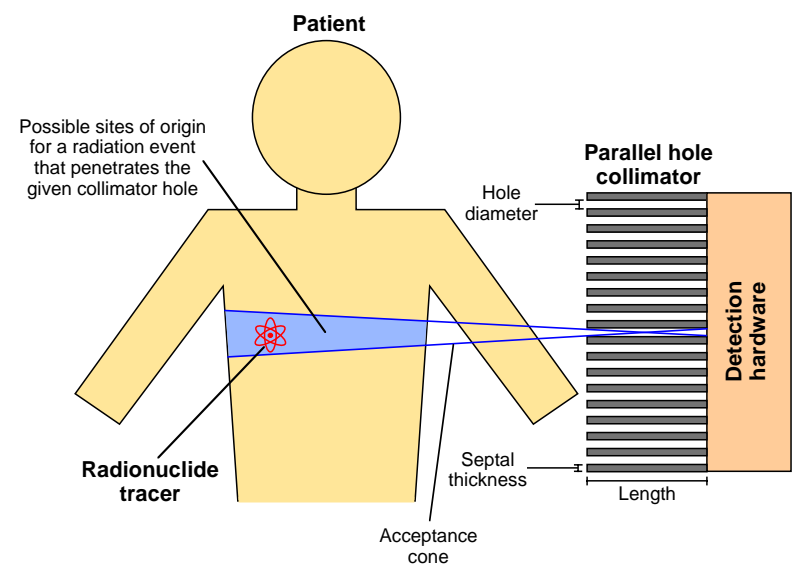

Figure 1.2: A collimator is used to localize the distribution of radionuclide activity. By limiting the possible site of origin for each detected event to a narrow cone, a parallel hole collimator provides information on the location of the radionuclide tracer within the patient. Increasingly narrow collimator acceptance cones improve spatial resolution at the expense of sensitivity. This presents the localization process from the perspective of the detector (once an event is detected, where did it come from?), but it is also possible to view localization from the perspective of the source (given an event originating at a specific point, what part of the detector is likely to observe it?).

information regarding its distribution. By altering the geometry such that the collimator acceptance cones are narrower, it is possible to improve collimator spatial resolution. However, narrower acceptance cones also mean that fewer events pass through the collimator and reach the detection hardware, thereby decreasing sensitivity. The geometric efficiency of a parallel hole collimator is described mathematically in Equation $1.2[12,13]$ :

$$
g \approx K\left(\frac{d}{l_{e}}\right)^{2}\left(\frac{d}{d+t}\right)^{2}
$$

where $g$ is the collimator geometric efficiency (expressed as the fraction of gamma rays emitted by the source that successfully pass through the collimator), $d$ is the hole diameter, $l_{e}$ is the effective length of the collimator (which is shorter than the physical 
length due to septal penetration), $t$ is the septal thickness, and $K$ is a constant that depends on the hole shape $(\sim 0.24$ for circular holes, $\sim 0.26$ for hexagonal holes, and $\sim 0.28$ for square holes). The effective collimator channel length is approximately given by [12]:

$$
l_{e} \approx l-2 \mu^{-1}
$$

where $l$ is the true physical length of the collimator and $\mu$ is the attenuation coefficient for gamma rays of the characteristic energy in the collimator material (e.g., lead).

The collimator spatial resolution for the same parameters is given by $[12$, $13]:$

$$
R_{\text {collimator }} \approx d\left(\frac{l_{e}+b}{l_{e}}\right)
$$

where $b$ is the distance between the collimator and the object being imaged. Combining Equations 1.2 and 1.4 produces the following key trade-off:

$$
g \propto\left(R_{\text {collimator }}\right)^{2} .
$$

Thus, in general collimator spatial resolution can only be improved by decreasing collimator efficiency, and efficiency can only be improved by worsening spatial resolution. Because this trade-off significantly limits system performance, it must be carefully considered in the design of any single photon device employing a collimator.

Equation 1.4 also brings forth another key point regarding collimatorsspatial resolution worsens with imaging distance in an approximately linear fashion. For planar imaging this means that objects closer to the collimator will be more clearly defined than will those at greater imaging distances, motivating the placement of the camera as close to the tissue of interest as possible. For SPECT imaging this characteristic of the collimator is yet another phenomenon that must be accounted for in the reconstruction algorithms to achieve the highest quality images possible.

Finally, while these considerations have focused exclusively on parallel hole collimators, single photon devices can also can use converging, diverging, fanbeam, and pinhole collimators. The basic efficiency versus spatial resolution trade-off illustrated by Equation 1.5 still holds true, however, as does the general worsening of 
spatial resolution with increased imaging distance. The motivation for using such collimator geometries, however, is to trade off spatial resolution and field of view.

\subsubsection{Statistical Noise}

The number of detected radiation events used to generate images in single photon imaging is relatively small, primarily because the extremely low geometric efficiency afforded by an absorption collimator. Given this limitation, the two most obvious means of increasing the number of detected events would be to increase the activity in the field of view (FOV) or to image the patient for a longer period of time (or both). However, the activity that can be safely administered to the patient is limited by concerns over the radiation dose that will be delivered by the radionuclide tracer, and the time that can be allotted for an imaging study is in practice limited by the patient's growing discomfort and by his or her ability to remain near-motionless for extended periods of time. The end result is that the density of events recorded in single photon studies is typically in the range of $100-3000$ counts $/ \mathrm{cm}^{2}$, drastically less than the $\sim 10^{8}$ counts $/ \mathrm{cm}^{2}$ achieved in photography or X-ray radiography [13]. Single photon studies - with a total number of detected events typically in the 100,000s for planar imaging or perhaps 1,000,000s for SPECT - are thus photon-starved and suffer reduced image quality as a result.

The statistical noise experienced by individual pixels (or photodetectors) in a single photon camera is best expressed using the Poisson distribution. The standard deviation in the number of counts detected by a given pixel is thus given by:

$$
\sigma \approx \sqrt{N}(\text { for Poisson distribution })
$$

where $N$ is the number of events detected in that pixel. For values of $N$ near 100 , which is typical of many single photon studies, this statistical uncertainty is a significant percentage of the measured number of counts and can obscure small or difficult-to-see features. This problem is further exacerbated in SPECT, where the 
reconstruction algorithms generally amplify this noise in the final image [14].

\subsubsection{Attenuation}

Attenuation refers to the loss of gamma rays as they travel from their sites of origin within the patient to the camera's collimator and detection hardware. This loss of photons occurs as a result of photon-matter interactions in the patient, namely photoelectric absorption and Compton scattering. Attenuation can be described mathematically in very simple terms:

$$
I(x)=I(0) e^{-\mu x}
$$

where $I(x)$ is the intensity of the unattenuated gamma photons after travelling a distance $x$ through the absorbing matter (thus $I(0)$ is the original intensity of the gamma rays) and $\mu$ is the attenuation coefficient. The value of $\mu$ accounts for both photoelectric and Compton effects and is a function of both gamma ray energy and the host material. Expressed a different way, $e^{-\mu x}$ is the probability that a photon will not have scattered or been absorbed after travelling a distance $x$ in the given material. For the $140 \mathrm{keV}$ emissions of ${ }^{99 m}$ Tc travelling through water (an excellent approximation for soft tissue), the value of $\mu$ is about $0.15 \mathrm{~cm}^{-1}$, meaning that $50 \%$ of the gamma rays will be either absorbed or scattered after travelling $4.6 \mathrm{~cm}$.

In planar imaging attenuation is deleterious because it makes features deeper within the patient more difficult to see. Hence, a tumor that would be detected near the surface of the skin may go undetected if it is shielded from the camera by several centimeters of tissue. For SPECT imaging the situation is even worse in that attenuation causes significant distortion of final, reconstructed image. More centrally-located tissue is underrepresented because for the same activity density, fewer photons will reach the detector from those tissues than from surface tissues. This effect must be corrected for, often by using an attenuation map derived from radioisotope transmission sources, a transmission X-ray computed tomography (CT) scan, or calculations using geometrical shape and assuming uniform attenuation. 


\subsubsection{Scatter and Energy Resolution}

Compton scattering, one form of attenuation mentioned in the previous section, occurs when a gamma ray collides with a loosely-bound outer shell electron, transferring some of its energy to the electron and thereby ejecting it from its parent atom. The end result is that the incident photon is deflected by a certain angle and continues on its way with decreased energy, while the ejected Compton recoil electron is now free and has been imparted with a certain kinetic energy. The amount of energy transferred depends on the angle, $\theta$, through which the incident photon is deflected:

$$
E_{\text {scattered }}=\frac{E_{\text {incident }}}{1+\left(E_{\text {incident }} / 0.511\right)(1-\cos \theta)}
$$

where $E$ represents the incident and scattered photon energies in $\mathrm{MeV}$.

Scattered photons are problematic because after changing directions they can still be detected by the camera and incorrectly incorporated into the image data. Collimators provide directional information by assuming a straight-line flight path from the point of origin, an assumption that is obviously violated by Comptonscattered photons that happen to be detected. The end result is that Compton scattering causes incorrect information to be incorporated into the image, thereby blurring the image and potentially decreasing the contrast of critical structures.

One means of dealing with scattered photons is energy discrimination. As evidenced by Equation 1.8, photons lose energy when they are deflected, making it possible to remove scattered photons from the image data if their measured energies are sufficiently less than the characteristic energy of the radionuclide in use. The ease with which scattered photons can be rejected obviously increases the greater the difference between the energy of the scattered photons and the characteristic emission energy of the radionuclide. Further, the more accurately the detection hardware can measure the energy of gamma rays (i.e., the better the energy resolution of the camera), the easier it is to distinguish scattered from unscattered gammas.

The detector system's energy resolution is usually expressed as a fwhm 
percentage of the mean photon energy, as shown in Figure 1.3. The energy acceptance window used for energy discrimination is defined based on the characteristic energy of the radionuclide, the energy resolution of the detector, and the needs of the specific application. Photons with measured energies outside of this acceptance window are assumed to be either background radiation or Compton-scattered photons and are rejected. A narrower acceptance window obviously allows a greater number of these undesirable gamma rays to be rejected, but also causes the rejection of a greater number of unscattered radionuclide gammas as well (note the Gaussian tails in the photopeak in Figure 1.3 which result from unscattered gammas). The better the energy resolution of the camera, however, the narrower the energy discrimination acceptance window can be (thus allowing rejection of additional Compton-scattered and background photons) without removing an excessive number of the desirable gamma rays from the image data.

\subsection{Prospects for the Proposed Gamma Camera}

The compact, discrete gamma camera technology described in this thesis is designed to provide small, lightweight, inexpensive devices that efficiently detect and image tumor-avid radiopharmaceuticals. A hexagonal hole lead collimator provides directional information, discrete $\mathrm{CsI}(\mathrm{Tl})$ crystals convert incident gamma rays to visible scintillation light, and a custom, low-noise photodiode array coupled to the $\mathrm{CsI}(\mathrm{Tl})$ crystals detects these scintillation photons and produces electric signals that are subsequently read out by a custom integrated circuit (IC). Finally, a computer collects and processes the data produced by the ICs and generates an image of the tracer distribution. This design is summarized in Figure 1.4.

Cameras constructed using this technology and designed for breast imaging are intended to replace the traditional Anger cameras currently used in scintimammography studies. Anger cameras are not optimized for imaging the breast and are suboptimal for this application primarily because their large and bulky size prevents 


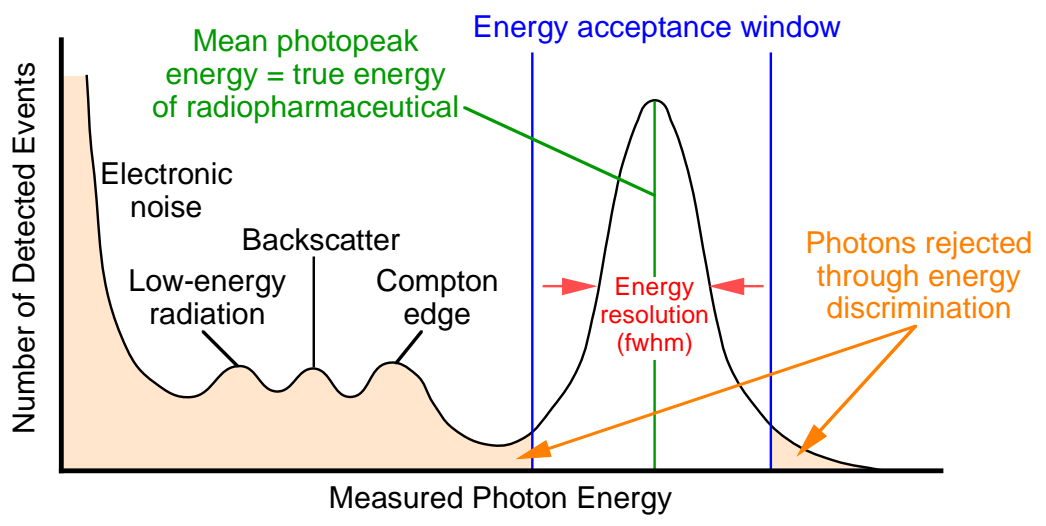

Figure 1.3: Photopeak and corresponding energy resolution for a monochromatic source. This histogram shows the typical response of a single photon camera to gamma rays of a specific energy (e.g., the $140 \mathrm{keV}$ emissions of ${ }^{99 m} \mathrm{Tc}$ ). Photons with measured energies outside of the energy acceptance window are assumed to be either background radiation or Compton-scattered photons and are not incorporated into the image data.

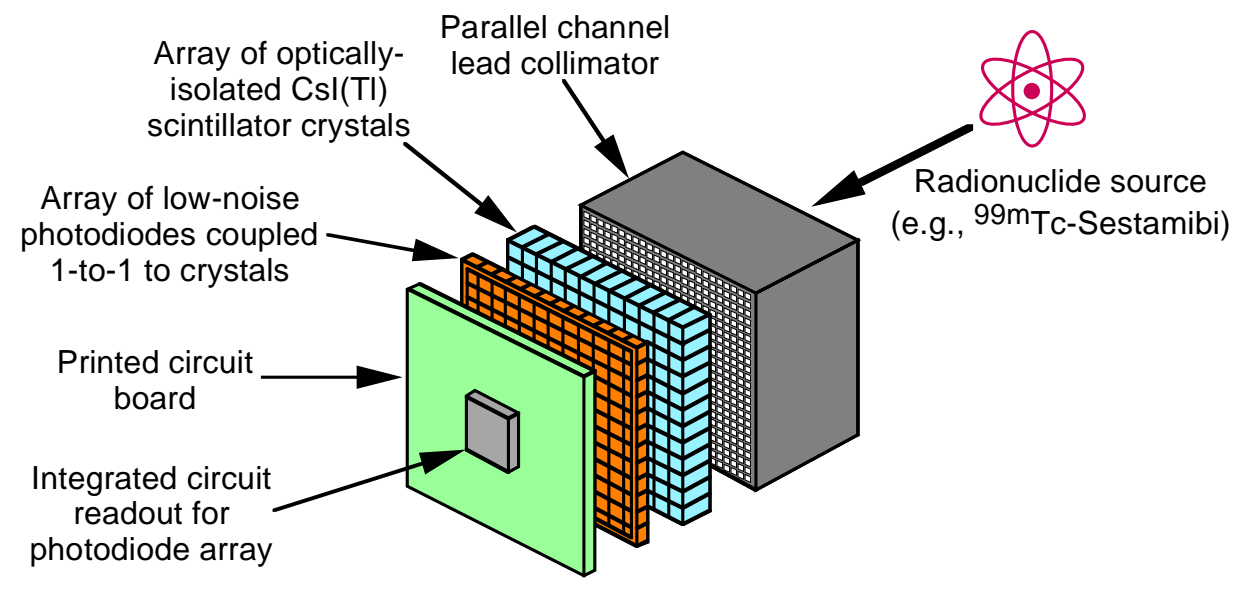

Figure 1.4: Basic components of a compact, discrete $\mathrm{CsI}(\mathrm{Tl})$ scintillator/Si photodiode gamma camera with custom IC readout. 
easy access to desired imaging areas. The significant size of Anger cameras results in part from the use of large photomultiplier tubes (PMTs) as well as the need to thoroughly encase the $\mathrm{NaI}(\mathrm{Tl})$ scintillator block to protect it from moisture. With the proposed technology the PMTs are replaced by extremely compact photodiode arrays and the $\mathrm{NaI}(\mathrm{Tl})$ is replaced by much less hygroscopic $\mathrm{CsI}(\mathrm{Tl})$, thereby eliminating both of these problems. The details of both these and other single photon camera technologies are explored more thoroughly in Chapter 3.

\subsubsection{Advantages}

The major advantages provided by the compact, discrete gamma camera relative to traditional Anger cameras include:

- The small camera size allows shorter imaging distances, improving collimator spatial resolution.

- The compact design permits a greater variety of viewing angles and allows multiple cameras to take different views simultaneously.

- Arrays of small photodiodes provide improved intrinsic spatial resolution.

- The multiple scintillator-photodiode channels yield a higher overall maximum event rate.

- The camera should ultimately prove inexpensive relative to traditional Anger cameras.

\subsubsection{Specific Applications}

Scintimammography with the new camera is intended to complement Xray mammography as a means of detecting breast cancer and verifying suspicious lesions, improving diagnostic accuracy and allowing a reduction in the present false positive rate of about $75 \%$ [15]. Because of its high sensitivity $(\sim 90 \%)$ and low cost, 
X-ray mammography will remain the mass screening tool of choice for some time, but X-ray mammography does have some important limitations that make other imaging modalities valuable adjuncts. Scintimammography with the new compact gamma camera has the potential to be particularly useful for tumor detection and verification in the following cases:

- women with dense and glandular breasts (often younger patients) where the sensitivity of X-ray mammography is impaired

- post-surgery discrimination between tumor recurrence and scar tissue, because scarred breasts are poorly imaged with X-ray mammography

- evaluation of tumor response to chemotherapy as such treatment progresses

- noninvasive evaluation of axillary lymph node involvement

- detection of the sentinel node associated with small breast tumors

- pre-surgical evaluation of multicentric disease

- evaluation of the efficacy of new radiopharmaceutical agents (e.g., labeled growth factors).

Breast cancer evaluation and diagnosis, including the use of various imaging modalities under different circumstances, is covered in detail in Chapter 2.

Finally, this compact gamma camera technology is highly flexible and with straightforward modifications could be used to construct medical imaging devices of almost any size. The compact nature of the imaging system make it particularly well suited for organ-specific imaging applications including, for example, devices designed to detect prostate cancer. 


\subsection{Research Overview}

As described in Section 1.3, the purpose of this research is to develop compact, discrete $\mathrm{CsI}(\mathrm{Tl})$ scintillator/Si photodiode camera technology for use in breast cancer imaging. Chapter 2 describes breast cancer morphology, diagnosis, and treatment in detail, beginning with the pathology of the most important benign and malignant disorders. The strengths, limitations, and uses of both X-ray mammography and biopsy procedures are explored, leading into discussion of the complementary roles that other imaging modalities - ultrasound, contrast-enhanced magnetic resonance imaging (MRI), positron emission mammography (PEM), and scintimammography do and in the future potentially will play in breast cancer screening and diagnosis. Based on the available literature I have generated a decision-making tree indicating when different techniques, including scintimammography with compact gamma cameras, are likely to be performed.

The fundamentals of single photon gamma cameras are described in Chapter 3, beginning with the traditional Anger camera that was developed decades ago but which continues to be a powerful force in medical imaging. The discussion that follows includes the advantages and challenges of discrete scintillator/photodiode cameras, motivating this thesis and laying out the specific technical hurdles that must be addressed with such a design. Other new competing technologies, namely solid-state CdZnTe cameras and position sensitive photomultiplier tube (PSPMT) cameras, are presented as well.

Chapter 4 presents some background on electronic noise sources in chargesensitive preamplifiers and then focuses on the performance of a 12-pixel prototype $\mathrm{CsI}(\mathrm{Tl})$ scintillator/Si PIN photodiode imaging system that I constructed and characterized. The results are some of the first to demonstrate that this technology is viable and have helped pave the way to the more advanced imaging systems discussed later in the thesis.

Chapter 5 summarizes the results of Monte Carlo simulations of breast 
tumors imaged with a compact, discrete gamma camera. I designed and developed these simulations to assist in making intelligent design decisions regarding pixel size and collimator configuration, as well as to explore the influence that factors such as energy resolution, tumor size, and tumor-to-background uptake ratio have on the resulting image characteristics.

The custom IC readout that is critical to achieving a compact, modular camera design is presented in Chapter 6. Details include the basic design and function of the IC as well as its capabilities and limitations. In addition to designing and assembling the testing set-up so that I could measure and report on the final IC performance, I also specified IC characteristics and tested and debugged early prototype ICs. The latter effort is highlighted through discussion of the features that were changed in the final version of the IC.

In Chapter 7 I characterize the final low-noise 64-pixel photodiode arrays, then present my final design and summarized assembly procedures for complete 64pixel imaging modules. These are the critical building blocks for cameras utilizing an array of such modules, and the goals of compact design and of attaining performance characteristics take center stage. I further present the design of a camera currently under construction which consists of an array of 16 modules and which provides sufficient imaging area for scintimammography studies. Finally, I present some characterization measurements made on a single 64-pixel module.

Finally, all of this work and conclusions related to it are summarized in Chapter 8. I recommend future research directions for this gamma camera technology, including both improvements to the individual module components as well as possible courses of development for cameras as a whole. The ultimate goal, of course, is using this technology in clinical scintimammography trials. 


\section{Chapter 2}

\section{Breast Cancer Morphology, Diagnosis, and Treatment}

Death rates from breast cancer declined significantly during 1992 to 1996...This decline is probably the result of better detection and improved treatment.

-American Cancer Society

\subsection{Pathology of Breast Cancer}

Cancer is a group of diseases wherein some of the body's cells no longer demonstrate normal restraints on growth, allowing the diseased cells to expand out of control and incidentally threaten healthy tissues. Although there are a few exceptions, most cancers generate a solid mass of rapidly-growing cells known as a tumor. The patient's life is generally most threatened when and if the cancer spreads - a process known as metastasis - because malignant tumors may then recklessly grow in multiple and varied locations throughout the body. Many patients suffering from advanced (and metastasized) breast cancer, for example, face the greatest threat from tumors growing in the lungs or bones. Different types of cancer are named for 
the organ which originally spawned the diseased cells because the progenitor cells largely determine the nature of the cancer, including key characteristics such as how aggressively it grows and how it responds to treatment.

Section 1.1 presented some striking statistics on the severity of breast can-

cer. Both its imposing prevalence among the general female population and its lethality when left untreated through preliminary growth stages have earned this disease a great deal of attention. Breast cancer, however, is treatable with an extremely high survival rate if it is detected in the early stages of development prior to metastasis (and hence the growth of satellite tumors outside the breast). Thus there is strong motivation for the implementation of comprehensive screening tests and the development of improved methods of detection and disease staging. While these efforts are rightly focused almost entirely on female patients, breast cancer can occur in men as well. Male breast cancer, in fact, is almost always fatal, but fortunately is extremely rare.

This chapter describes the basic structure of the healthy female breast, the nature of the various diseases - both benign and malignant - that frequently develop, and the nature of breast cancer detection and treatment. Screening and examination techniques from conventional X-ray mammography and biopsy to the more experimental scintimammography and magnetic resonance imaging (MRI) of the breast are discussed. The chapter concludes by summarizing the complementary roles that individual imaging, detection, and staging methods currently do and, with further development (e.g., scintimammography using new compact, discrete scintillation camera technology), will play in identifying breast cancer.

\subsubsection{The Normal Breast}

One of the most important structures in the normal female breast is the mammary gland, which produces milk during lactation to nourish an infant. This gland consists of approximately 15 to 25 lobes, each of which is in turn comprised of a 


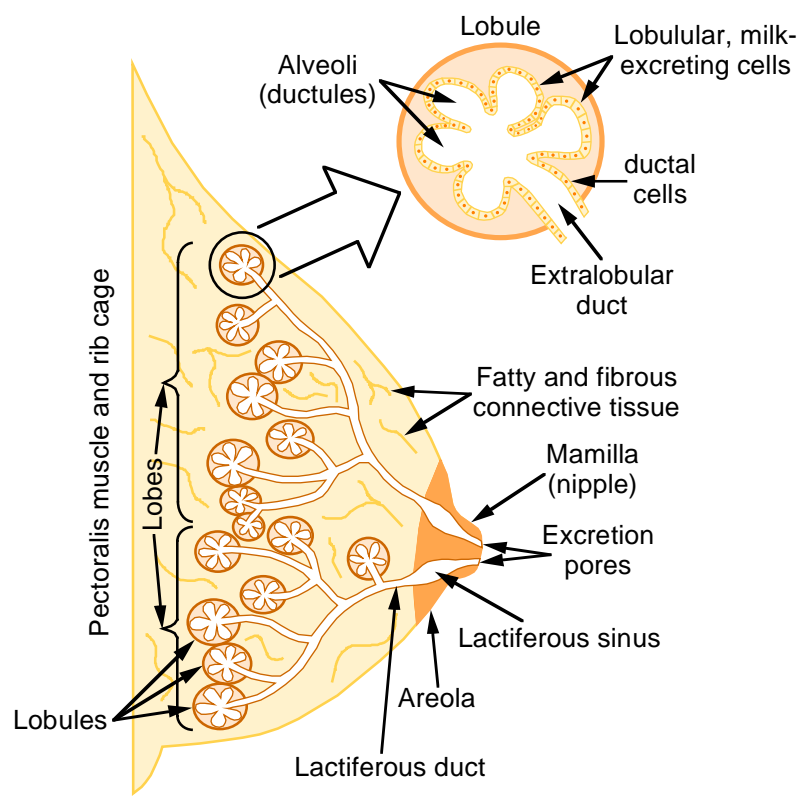

Figure 2.1: Structure of the normal female breast. During lactation the lobular cells in the mammary glands produce milk, which is carried by the ductal system to the mammilla (nipple). Figure adapted from [16].

ductal system and numerous lobules. The lobules form the termination of the mammary gland's ductal system - they are relatively spherical structures formed from the cells that actually produce the milk. These secretion cells are organized into rounded alveoli which open into the smallest branches of the lactiferous ducts, allowing milk to flow freely from the lobules into the ductal system and then ultimately to the nipple where it can be excreted. Mammary glands vary widely in size and shape, but they tend to be symmetrical across the two breasts and typically demonstrate the greatest presence in the upper, lateral quadrants of the breasts. The breast tissue surrounding the mammary gland consists of fatty tissue, networks of blood and lymph vessels that sustain the glandular cells, and fibrous ligaments that provide physical support by connecting the gland to both the skin and to the fascia covering the pectoralis muscles. The structure of the breast is summarized in Figure 2.1. 
The structure of the mammary gland varies significantly with age and during pregnancy and lactation. At birth the gland is primarily a network of lactiferous ducts devoid of any alveoli, but during puberty the gland responds to ovarian oestrogenic hormones, developing significant branching of the ducts and forming precursor-alveoli structures. True milk-secreting alveoli do not appear until pregnancy, however, when oestrogen and progesterone levels rise. During pregnancy this growth of the glandular tissue, coupled with an increase in adipose tissue and the development of a more extensive blood vessel network, causes temporary enlargement of the breasts. A number of months after pregnancy when lactation ceases, the glandular tissue returns to a more "relaxed" state and the alveoli shrink. After menopause, the mammary gland atrophies and the alveoli and ducts begin to degenerate and disappear, though some of the former structure remains. In general aging also tends to replace the breast's fibrous connective tissue with adipose tissue, a process that not only changes the appearance of the breasts but which, as described in Sections 2.2.3 and 2.4.5, can also influence the detection of breast cancer.

A final structure of importance in the breast is the lymphatic system, which collects tissue fluid (eventually known as lymph) and returns it to the venous part of the cardiovascular system. Lymph nodes are small organs that are located along the lymphatic ducts and which serve to destroy microorganisms and metastasized cancer cells in the lymph, preventing them from using the lymphatic ducts to spread throughout the body. Sometimes, however, lymph nodes will be overwhelmed by cancer cells trapped in the nodes which continue to grow and multiply out of control. The axillary lymph nodes, located near the armpit, lie along the lymphatic vessels that drain fluid from most of the breast and are therefore often the first place where metastasized breast cancer cells strike. As will be discussed later in this chapter, the status of the axillary lymph nodes (healthy versus invaded by metastasized cancer cells) is a powerful prognostic factor in determining how advanced the breast cancer in a particular patient is. Malignant tumors originating in the medial part of the breast, however, also have the possibility of metastasizing to the internal mammary 
lymph nodes.

\subsubsection{Carcinoma in Situ}

Carcinomas in situ are lesions that display cancerous characteristics but that have not penetrated the membrane surrounding their site of origin (known as the basement membrane). As such, they cannot metastasize and in and of themselves do not pose an immediate risk to the patient's health. A significant percentage of the much more dangerous invasive carcinomas (see Section 2.1.3), however, are believed to develop from carcinomas in situ. Carcinoma in situ, while not a necessary precursor to the development of invasive carcinoma, is thus an indicator of increased risk. About $90 \%$ of all carcinomas in situ contain microcalcifications and are therefore good candidates for detection with X-ray mammography. This disease is commonly classified into lobular carcinoma in situ (LCIS) and ductal carcinoma in situ (DCIS), depending on the type of cells that gave rise to the lesion.

LCIS tends to grow slowly within a lobule and can remain unchanged for many years, leading some to regard "carcinoma" as a misnomer and instead classify this condition as pre-malignant rather than as a true cancer. Typically LCIS is very difficult to diagnose either clinically or mammographically, hence it is most commonly identified during biopsies performed because of questionable palpation or X-ray mammography results [9]. LCIS is multicentric (demonstrates lesions in more than one duct system) nearly $50 \%$ of the time, is bilateral (present in both breasts) about $30 \%$ of the time, and has been shown to represent a ten-fold increase in the patient's risk for developing invasive carcinoma [9].

DCIS grows within the ductal system of the mammary gland and, while generally not palpable, typically produces microcalcifications which allow mammo-

graphic detection. Noncalcified DCIS lesions, however, are sometimes identified as a result of biopsies performed for other reasons. About $30 \%$ of DCIS cases are multicentric [9]. DCIS has historically been treated with mastectomy (removal of the 
entire breast), so the available data are not conclusive as to the risk for developing invasive carcinoma if breast-conserving treatment is employed. One study suggests, however, that $30 \%$ of untreated DCIS will progress into invasive carcinoma within 10 years [17].

\subsubsection{Invasive Carcinoma}

Invasive carcinoma is the most common malignancy in women and is the primary cause of the many deaths wrought by breast cancer. Unlike carcinoma in situ, invasive carcinoma does break through the basement membrane, allowing the cancer to spread into surrounding tissue and giving rise to the possibility of metastasis. Because of this possibility, it is highly desirable to identify and treat invasive carcinoma before the lesions have much time to grow and metastasize. It has been shown, for instance, that prognosis significantly worsens once tumors reach $15 \mathrm{~mm}$ in size [18]. Invasive carcinomas demonstrate a great deal of variability in their presentation and growth patterns, making it challenging to identify the lesions in early stages of growth.

Like carcinomas in situ, invasive carcinomas are categorized into lobular and ductal lesions. Invasive ductal carcinoma is the more frequent, accounting for 75-80\% of invasive breast carcinomas. Lesions of this type often exhibit a spiculated growth pattern (i.e., multiple spiked appendages growing outward from a central location) and contain microcalcifications about $30-40 \%$ of the time [9]. While this is indeed a very serious type of cancer, it fortunately tends not to spread from the original breast to the second, making it easier to successfully treat the patient if the cancer is caught early enough.

Lobular carcinoma accounts for only about 5-15\% of invasive carcinomas, but it is more deadly than invasive ductal carcinoma. Invasive lobular tumors are often discovered late (leading to worsened prognosis) because they form no microcalcifications and tend to have a diffuse growth pattern. Tumors of this type are often 
multicentric or bilateral and have a propensity for recurring in the opposite breast after treatment [17].

Other invasive carcinomas that clinicians must be aware of when screening and diagnosing patients include: medullary carcinoma, mucinous carcinoma, papillary carcinoma, cribriform carcinoma, tubular carcinoma, Paget's carcinoma, and inflammatory carcinoma. These disorders are quite rare and vary substantially in presentation and prognosis. Medullary, mucinous, and papillary carcinomas are, in fact, special types of invasive ductal carcinoma that demonstrate unique characteristics.

\subsubsection{Cysts}

Cysts are hollow, fluid-filled sacs that account for the majority of masses found in the female breast. About half of women between 30 and 40 years of age develop cysts, which can range in size from non-palpable to several inches across [9]. Cysts are very rarely associated with breast cancer and are considered to be extremely benign, though they can become painful just prior to menstruation. Breast cancer screening, detection, and diagnosis must take cysts into account because palpation and X-ray mammography of cystic structures often proves unable to distinguish cysts from more serious conditions. Under these conditions ultrasonography is typically employed in order to determine whether the suspicious structure is indeed cystic (see Section 2.4.3). Cysts themselves require none of the treatment procedures associated with breast cancer but are occasionally aspirated, in which case a needle is used to remove the liquid and thereby allow the lesion to collapse.

\subsubsection{Other Benign Disorders}

While most cancers generate tumors, not all tumors are cancerous. There are a number of benign diseases triggered by hormone imbalances that generate tumors but that do not appreciably threaten the patient's health. These conditions 
often require no treatment themselves, but about 30\% of all benign breast disorders do indicate an increased risk for developing breast cancer in the future [9]. Like cysts, they play an important role in breast cancer detection and diagnosis schemes because they can be confused with malignant tumors. It is undesirable to unnecessarily biopsy every patient with a benign tumor, but obviously it is critical not to misdiagnose an invasive carcinoma as a benign disorder and thereby let it grow unchecked.

Fibroadenomas are by far the most common breast tumors, occurring in all age groups but found predominantly in young women in their teens, twenties, and early thirties. These benign tumors are composed of both fibrous (hence fibro) and glandular (hence adenoma) tissue, forming rounded solid masses about $1-3 \mathrm{~cm}$ in size that are often discovered by palpation [9]. Only about $0.1-0.3 \%$ of fibroadenomas prove cancerous, and in those cases the threat is usually posed by a carcinoma in situ located within the fibroadenoma [19]. Fibroadenomas are sometimes removed if they become painful or cosmetically unappealing.

Adenomas are excessive growth of the glandular tissue, specifically in either the lobules or adjacent ducts. While this condition is further categorized into a number of disorders, the most notable is sclerosing adenoma. This disease causes lobular tissue to grow and expand in a generally painful and non-palpable fashion. Unfortunately sclerosing adenoma is difficult to differentiate from cancer, so biopsy is usually required.

Papillomas are small growths in the lactiferous ducts near the nipple that account for slightly over $1 \%$ of all breast tumors. Because of their location within the ductal system, these tumors often cause watery or bloody discharge from the nipple. Certain types of papilloma are associated with an increased risk of developing malignancies, so diagnosis should consider both the number of tumors as well as their localization within the ducts.

Trauma and injury to the breast can also produce benign breast disorders. The most common are fat necrosis (death of fatty tissue) and hematoma (accumulation of blood), both of which appear as lumps. Fat necrosis in particular forms a hard 
mass, making it easy to confuse with malignancy and therefore necessitating biopsy. Ironically, the breast injury caused by biopsy or surgical intervention can produce these conditions, but there is no evidence that they increase the risk of cancer.

There are many other benign breast disorders with specific characteristics that the clinician must be aware of, but this sampling covers some of the most important. Certainly it indicates the wide variety of disorders encountered and the challenge facing doctors who must differentiate between benign and malignant tumors in determining an appropriate course of treatment.

\subsection{Detection and Diagnosis of Breast Cancer}

\subsubsection{Physical Exam}

Physical exam of the breasts includes visual inspection and palpation, and should be performed monthly by the patient at home and by a doctor as part of any breast exam (e.g., yearly screening). Breast size and symmetry, contour, skin changes, presentation of the nipples, and the presence of any palpable masses all can point toward a malignant tumor. Some $10 \%$ of breast cancers are detectable only by physical examination, most notably those in the periphery of the mammary gland or the axillary tail where they may not show up on an X-ray mammogram because they lie outside the field of view [9].

\subsubsection{X-ray Mammography and Screening}

Planar X-ray mammography is the single most important imaging modality for detecting and diagnosing breast cancer. Its relatively low cost, high spatial resolution $(\sim 100 \mu \mathrm{m})$, and high sensitivity for detecting tumors in early growth stages make it useful both for diagnosing symptomatic patients as well as for screening the population at large who do not demonstrate any suspicious symptoms. In fact, X-ray mammography is the only imaging technique that has proven appropriate for screen- 
ing, and while there has been some contemporary controversy over the appropriate age to begin screening, the general consensus is that women over 40 should have an annual mammogram. Yearly screening with X-ray mammography has been shown to reduce breast cancer mortality by $30-70 \%$ [9].

X-ray mammography involves positioning the breast between two plates and gently compressing, followed by the emission of an X-ray burst that penetrates the breast and is measured on the far side by an X-ray film-screen system. Generally two views of the breast are taken - the craniocaudal view (from the top looking down) and the mediolateral oblique view (looking from the center of the body outward at a downward angle). Finally, a lead grid is often employed just in front of the X-ray film-screen in order to reduce the number of photons scattered within the breast that reach the film. The X-ray mammography imaging process is summarized in Figure 2.2.

The X-ray energy spectrum used in mammography must be chosen with care because it affects both the patient radiation dose and the image contrast. In fact, the energy spectrum used is adjusted on a patient by patient basis in order to maximize contrast, as breast size and density affects the imaging problem. Generally low-energy X-rays allow small density differences in soft tissue to be imaged with higher contrast, but if the photon energy is too low the $\mathrm{X}$-rays will not penetrate the breast enough to form a useful image (especially true in large or dense breasts). Additionally, because the X-ray source is not monoenergetic but rather presents a range of energies generated by both characteristic and bremsstrahlung radiation, it is important to filter out the lowest X-ray energies because they contribute to patient dose without improving the image. Both the target/filter combination and the peak voltage of the vacuum tube used to generate the $\mathrm{X}$-rays can be adjusted to set the desired energy spectrum, with the peak X-ray intensity usually occurring between 15 and $20 \mathrm{keV}$. The most common target/filter combinations are molybdenum/molybdenum or tungsten/molybdenum, but molybdenum/rhodium, tungsten/rhodium, and rhodium/rhodium are also used. 


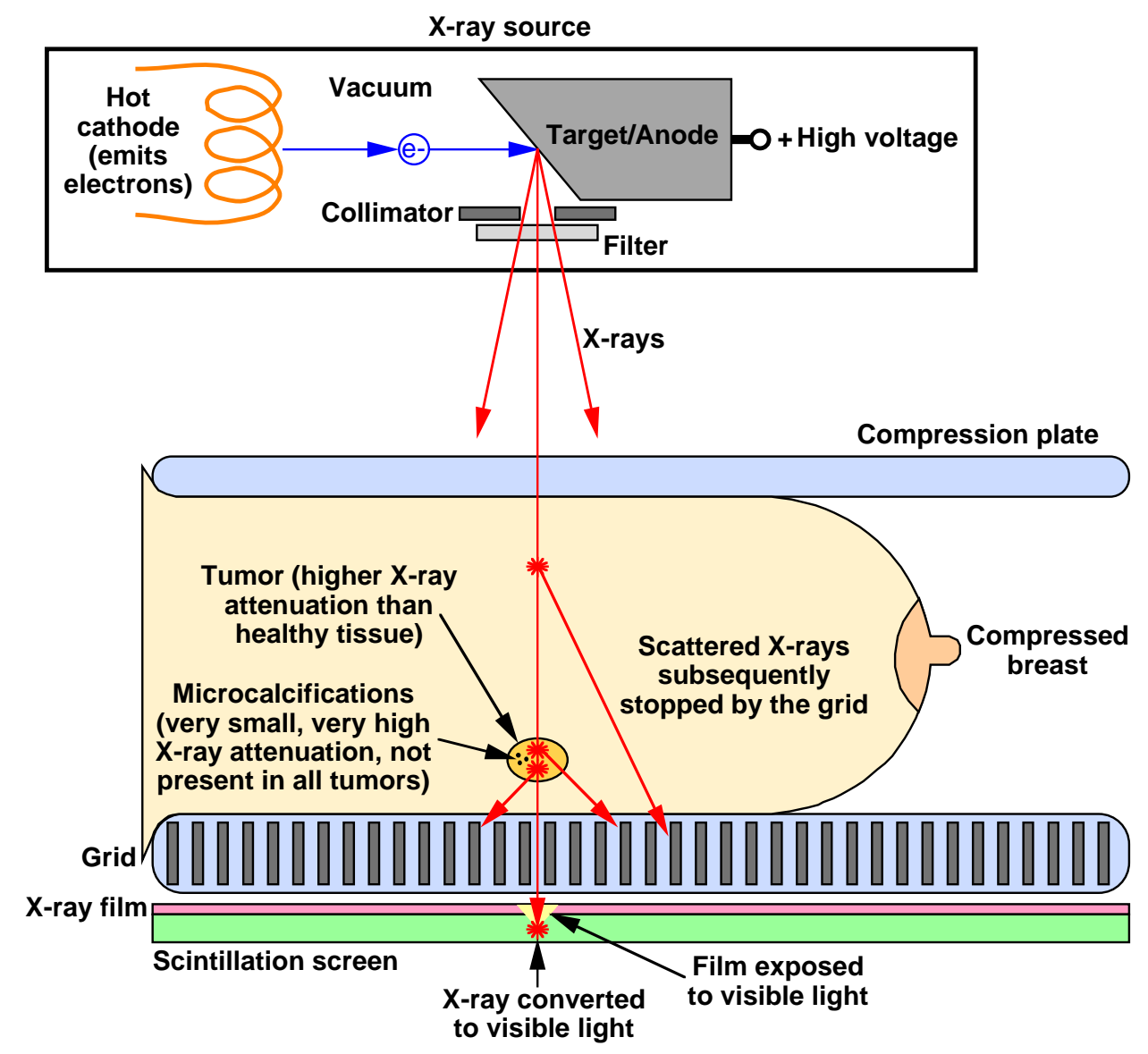

Figure 2.2: X-ray mammography involves compressing the breast and exposing it to $\mathrm{X}$-rays which penetrate the soft tissue and are detected on the far side of the breast by a film-screen system. A vacuum tube wherein electrons are accelerated into a target material is used to generate the X-rays. Figure adapted from [9]. 
One of the aspects of X-ray mammography of particular note to patients in general is breast compression, for often it is uncomfortable and attaches a negative stigma to the entire process. However, breast compression contributes significantly to the quality of the resulting image in multiple ways:

- Compression immobilizes the breast and thereby reduces motion blurring.

- It moves structures within the breast that will be imaged closer to the filmscreen system, reducing geometric blurring.

- The thickness of the breast is reduced, which increases the image contrast both by decreasing scattered radiation and by allowing less energetic radiation to be used.

- Decreased breast thickness also reduces the patient dose because a lesser depth of tissue must be penetrated.

- Compression helps distinguish malignant lesions from dense glandular tissue because healthy tissues tend to spread out whereas malignant lesions tend to persist as solid lumps.

- It equalizes the thickness of the breast across the image, reducing the necessary sensitivity range of the film.

Another patient concern is the radiation dose delivered to the breasts, which is in seeming contradiction with the goal of eliminating breast cancer! Glandular breast tissue is sensitive to radiation, and while this is certainly noteworthy, women who undergo X-ray mammography tend to be older and therefore tend to have more fatty and less glandular tissue in their breasts. The dose per breast for standard 2plane X-ray mammography is about 100-200 mrem, and it has been estimated that annual exposures at this level over a period of 20 years would, at worst, increase the risk of breast cancer from $10 \%$ to $10.06 \%$ [20]. This minor risk is far outweighed 
by the reduction in breast cancer mortality that is afforded by screening with X-ray mammography.

\subsubsection{Strengths and Limitations of X-ray Mammography}

X-ray mammography is the only imaging modality suitable for screening because it offers the following advantages:

- It has high sensitivity for small malignant lesions.

- Mammography is inexpensive and cost-effective, both because the instrumentation is relatively inexpensive and because it requires little physician time.

- Studies are reproducible and easy to document, making patient histories valuable and simple to produce.

- It alone images microcalcifications, which occur in $30-40 \%$ of invasive carcinomas and about $90 \%$ of carcinomas in situ.

As stated in Section 2.2.2, these strengths allow screening with X-ray mammography to achieve a 30-70\% reduction in breast cancer mortality.

While this modality is about $90 \%$ sensitive for the detection of breast cancer $[9,21,22,23]$, its specificity for distinguishing malignant from benign lesions is only $20-50 \%$ (i.e., there are many false positives) $[24,25,26]$. Thus a large fraction (well over $50 \%$ in most centers) of the suspicious structures identified in mammograms are non-cancerous and further diagnosis - usually by biopsy - is necessary before deciding whether to actually treat the patient for cancer [15, 23]. X-ray mammography has the additional drawback that its sensitivity for cancer is significantly decreased in patients with radiographically dense breasts (which can occur because of scarring or in patients under 50 who retain substantial glandular tissue) [9, 21, 27, 28, 29, 30]. Finally, the field of view afforded by X-ray mammography is limited to the tissue that can be positioned between the compression plates, making it difficult to detect lesions in the chest wall or in the axillary chain. 


\subsubsection{Biopsy}

Further diagnosis for mammography-positive women is very often obtained by biopsy. Histologic examination of the tissue harvested by biopsy is the gold standard for determining whether a suspicious structure is cancerous, and if so, what type of cancer it is. Two types of biopsy are commonly performed-fine-needle aspiration cytology and core-needle biopsy. The former is less invasive but because the latter removes small cores of tissue rather than simply aspirating cells, it provides more cells for examination and is less likely to outright miss the tumor in question. Side effects of biopsy include pain, stress, and minor bleeding and physical trauma. Further, biopsies tend to be time-consuming for the physician and are therefore associated with significant expense. This expense and patient discomfort are particularly noteworthy since only around $25 \%$ of the some 700,000 biopsies performed each year (most because of suspicious mammograms) reveal malignancies. This has led many breast cancer experts to conclude that better diagnostic imaging techniques are required in order to mitigate the specificity limitations of X-ray mammography and decrease the number of "unnecessary" biopsies.

A physician can target palpable masses near the surface of the breast with the biopsy needle "by hand," but in many cases biopsy must be guided by an imaging method. The two most common techniques are stereotaxic X-ray, wherein the needle is guided with the help of two simultaneous planar X-ray views that give a rudimentary 3-D perspective of the breast, and ultrasound. Research is also being performed on the possibility of using contrast-enhanced MRI, X-ray CT, and nuclear medicine imaging modalities to guide biopsy.

\subsubsection{Staging}

Pathologic staging is a critical part of breast cancer diagnosis because it is a strong indicator of which treatments are most appropriate and what the patient's chances for survival are. The most common staging system is known as the TNM 
scale because it is based largely on the Tumor size and histology, the status of the lymph Nodes, and whether the cancer has Metastasized outside of the breast and nodes.

The TNM scale ranges from 0 to IV, with higher numbers indicating more advanced cancers. Stage 0 diseases are either DCIS or LCIS and are very treatable with a 5-year survival rate approaching $100 \%$. Stage I cancers are small tumors less than $2 \mathrm{~cm}$ in diameter that demonstrate no nodal involvement, indicating a 5 -year survival rate of over $90 \%$. Once a tumor is larger than $2 \mathrm{~cm}$ the malignancy is usually classified as stage II and the 5-year survival rate drops to $65-85 \%$. Stage III lesions are larger than $5 \mathrm{~cm}$ and/or have begun to infiltrate the axillary nodes on the same side of the body, a relatively advanced disease state with a 5-year survival rate of only $45-55 \%$. Finally, the most advanced cancers are classified as stage IV and are characterized by frighteningly low 5 -year survival rates of $10-15 \%$. These malignancies are so deadly because they have metastasized to other parts of the body and have begun to grow in other organs. Clearly the best treatment options (see Section 2.3) vary widely with the stage of the disease.

\subsection{Treatment of Breast Cancer}

Once one or more malignant tumors are identified within the breast as outlined in Sections 2.2 and 2.4.5, a course of therapy must be implemented to combat the disease. Treatment for breast cancer is quite aggressive because the disease itself is so tenacious - any tumor cells left unchecked are likely to generate tumor recurrence and threaten the life of the patient. Breast cancer therapy can thus cause patients a great deal of pain and discomfort, and while the cure is undoubtedly better than the disease, courses of treatment must consider not only the likelihood of success but also the distress of the patient. The primary goals of treatment success and preservation of life depend upon a range of factors that include the type of cancer, stage of the disease, and patient history. 


\subsubsection{Surgical Therapy}

Most patients suffering from breast cancer will undergo some form of surgery in order to remove as much of the cancer as possible. If the lesion has yet to metastasize, for instance, it may be possible to completely halt the disease by simply removing the primary tumor. Since it is impossible to know if all malignant cells have been surgically removed, however, surgery is usually coupled with other techniques that increase the treatment's odds of success. The most common supplementary procedures are radiation therapy, chemotherapy, and hormone therapy, all of which will be discussed shortly.

Modified radical mastectomy (MRM) has been the most common surgical therapy for breast cancer for several decades. It involves the removal of the entire infected breast as well as many of the axillary lymph nodes associated with that breast. The advantage of this technique is that the removal of such a large volume of tissue increases the odds that all of the malignant cells are removed, making this procedure effective in treating a wide range of breast cancers in many stages of development. Additionally, the removal of the axillary lymph nodes allows for accurate staging of the disease. Clearly, however, it is traumatic and distressing to have an entire breast removed, and MRM is often followed by breast reconstruction surgery. Removing the axillary lymph nodes also has some morbidity associated with it, including the possibility of chronic weakness, edema, and pain.

Other forms of mastectomy include partial mastectomy, total mastectomy, and radical mastectomy. Partial mastectomy is the least invasive, removing only a portion of the breast (about 25\%). Total mastectomy involves the removal of the entire breast, but unlike MRM leaves the axillary lymph nodes in place. Radical mastectomy is the most serious surgery and entails the removal of everything in the vicinity of the tumor - breast, axillary lymph nodes, and chest wall muscles beneath the breast. MRM has essentially replaced radical mastectomy, however, because it has been shown to be just as effective in treating breast cancer but with less 
disfigurement and fewer complications.

While partial mastectomy does serve to preserve a large portion of the breast, the most breast-conserving surgery is the lumpectomy. This surgery involves the removal of the tumor as well as a layer of surrounding normal tissue, but leaves the rest of the breast intact. Although lumpectomy is desirable from a disfigurement standpoint, it is not always a valid option. It is contraindicated by multicentricity, for example. Breast-conserving surgery, be it partial mastectomy or lumpectomy, must be followed by radiation therapy if it is to be as successful in treating cancer as is MRM. Even with radiation therapy, however, cancer recurrence after lumpectomy can be as frequent as $25 \%$ if too little tissue is removed, as opposed to about $2-10 \%$ if sufficient tissue around the lesion is excised [17]. The percentage of breast cancer patients that undergo lumpectomy (vs. mastectomy) varies substantially between hospitals and appears to depend largely on physician preference and comfort with particular procedures [17].

\subsubsection{Axillary Node Dissection}

Axillary node dissection is a surgical procedure in which some of the nodes are removed from under the arm and histologically analyzed to determine the presence or absence of cancerous cells. This is critical to staging the development of the cancer and guiding treatment decisions because axillary lymph node status is the single most important prognostic indicator [31,32]. One study demonstrated that the five-year breast cancer survival rate dropped dramatically from $82 \%$ for patients with no infected nodes, to $47 \%$ for patients with five or six cancerous nodes, all the way down to $8 \%$ for patients with over twenty invaded nodes [33]. Dissection of the axillary nodes generally occurs at the same time as MRM or immediately following breast-conserving therapy. Removal of these nodes not only causes some additional surgical trauma, but is also associated with long-term lymphedema (swelling caused by excess fluid not removed by the lymphatic system) of the arm in as many as $60 \%$ 
of patients [33]. At the present time there is no accepted non-surgical method for evaluating axillary lymph node status, but advances in nuclear medicine and MRI imaging techniques may potentially change that in the future (see Sections 2.4.1, 2.4.2, and 2.4.4). This would be highly advantageous because it would aid therapy selection prior to surgical intervention.

A recent advance in the area of lymph node evaluation is sentinel lymph node biopsy. A radioactive material (usually $450 \mu \mathrm{Ci}$ of ${ }^{99 m} \mathrm{Tc}$ in a sulfur colloid) is injected into the area around the primary tumor along with blue dye. The radioactive material and dye are carried by the lymphatic system and accumulate in the first (sentinel) node they encounter in the axillary chain. A surgeon may thus identify this node by its radioactive signature and/or the blue coloration afforded by the dye. If the primary tumor has indeed begun to spread, the sentinel node is the most likely to contain metastatic cancer cells. Evaluation of the overall node status may therefore be performed by removing and analyzing only the sentinel node and leaving the others untouched, minimizing the possibility of complications such as lymphedema. If the sentinel node has been invaded by malignant cells, more nodes will likely be removed, whereas if the node is free of cancer further axillary surgery may be unnecessary. Detection of the radioactive material in the sentinel node is typically performed using a small radiation probe, but compact scintimammography cameras have begun to see use in the role at some medical centers [34].

\subsubsection{Radiation Therapy}

Radiation therapy is a localized treatment wherein a high dose (about 6000 rads) of radiation is delivered to the breast over a six to seven week period of time. This radiation bombardment is intended to kill malignant cancer cells in the breast and can be used either prior to surgery in order to shrink a tumor, or post-operatively in order to lower the likelihood of tumor recurrence. The exposure targets the greatest radiation intensity at the primary tumor or, if performed following lumpectomy 
or partial mastectomy, at the former site of the primary tumor. Radiation therapy has been shown to reduce the local occurrence of breast cancer after lumpectomy by 69-89\% [17]. In some cases radiation treatment may be directed at the axillary nodes as well. In addition to the obviously large radiation dose delivered to the patient's breast, side effects include swelling of the breast, erythema (sunburn-like changes in the skin), and fatigue.

\subsubsection{Chemotherapy}

Total body therapy comes in two forms - chemotherapy and hormone treatment - and is used to treat cancer cells that have spread anywhere throughout the body. These therapies can be used prior to surgery in order to shrink the primary tumor and make it easier to remove (neoadjuvant therapy), after surgery in order to reduce the likelihood of recurrence even if there is no direct evidence of metastasis (adjuvant therapy), or as the principal means of treatment if the cancer is known to have metastasized outside of the breast (primary therapy). Because these total body treatments operate somewhat indiscriminately, the toxic effects intended to hinder or kill malignant cancer cells can also prove harmful to the patient and provoke a variety of side effects.

Chemotherapy involves the administration of drugs that are especially toxic to dividing cells. These drugs are delivered by the bloodstream throughout the body, killing rapidly-dividing tumor cells and healthy cells alike (hence side effects such as hair loss). Therapy is given in cycles over a period of about three to six months and typically involves several drugs. The standard chemotherapy for adjuvant breast cancer treatment is cyclophosphamide, methotrexate, and fluorouracil (CMF), which results in an increase in survival rate of $8-12 \%$ compared to patients receiving no chemotherapy [17]. Side effects are usually temporary and include nausea and vomiting, higher risk of infection, loss of appetite, bladder irritation, fatigue, and the aforementioned hair loss. 


\subsubsection{Hormone Therapy}

Hormone therapy entails using a drug, commonly tamoxifen, that blocks the estrogen receptors on breast cells. This is advantageous because estrogen in the blood normally stimulates breast cancer cell growth, but when the estrogen receptors are blocked the cancer cells tend to remain dormant and do not multiply. Hence this treatment differs significantly from chemotherapy in that it does not actually kill malignant cells, but rather prevents them from growing by depriving them of hormonal stimulation. Tamoxifen is taken orally over a period of about five years and is used frequently in women with invasive carcinoma. It has been shown to increase the ten-year survival rate by over $6 \%$, with the greatest benefits observed in node-positive women [17]. Common side effects mimic menopause and include irregular menstrual periods, vaginal irritation, and hot flashes.

\subsection{Other Imaging Modalities}

\subsubsection{Scintimammography}

Recent research has demonstrated that scintimammography with tumoravid tracers (most commonly ${ }^{99 m}$ Tc-methoxyisobutylisonitrile, also known as sestamibi) and standard Anger gamma cameras can accurately diagnose primary breast cancer. Figure 2.3 depicts the basic scintimammography imaging situation. The patient is injected in with a quantity of radioactive tracer (typically $20 \mathrm{mCi}$ of the aforementioned ${ }^{99 m}$ Tc-sestamibi) that will be returned to the heart through the venous system and then distributed throughout the body. The injection is typically made in the contralateral arm or leg to prevent radionuclide tracer near the injection site from appearing in the breast image. Because of their high metabolic activity and well-developed vascularization, tumors will tend to collect more of the tracer per volume than will healthy tissue. Thus when the radioactive tracer decays and emits gamma rays, a larger number of these gammas will originate from tumor sites 


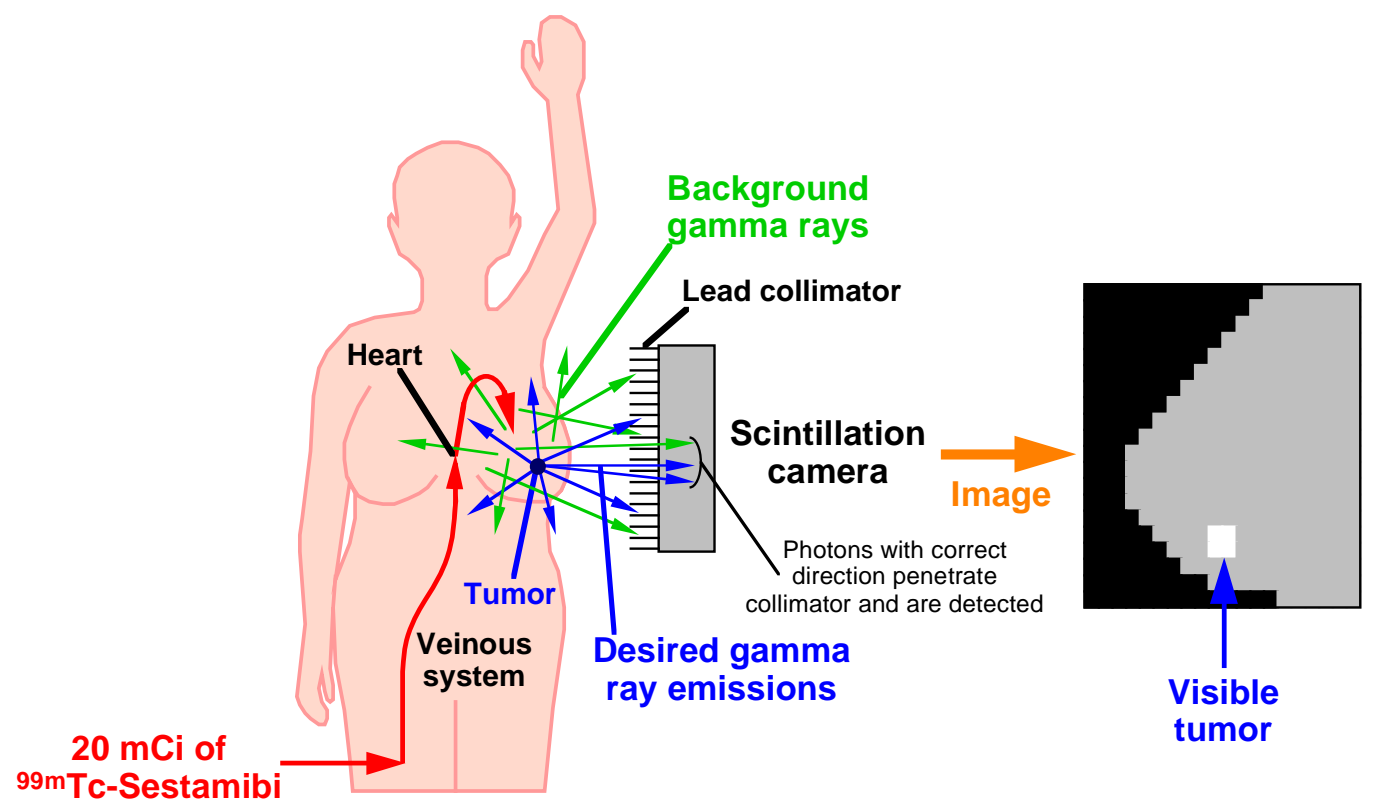

Figure 2.3: Scintimammography involves injecting the patient with a radioactive tracer (e.g., ${ }^{99 m} \mathrm{Tc}$-sestamibi) that will be distributed throughout the body by the heart. A scintillation camera equipped with a collimator observes the resulting gamma rays and generates a two-dimensional image of the tracer distribution in the breast, allowing visualization of any tumors present as "hot spots."

than from equal volumes of healthy tissue. A scintillation camera equipped with a collimator is used to observe the gamma rays and generates a two-dimensional image of the tracer distribution in the breast, allowing visualization of any tumors present as "hot spots." The total body dose for a $20 \mathrm{mCi}$ injection of ${ }^{99 m}$ Tc-sestamibi is about 330 mrem, but the effective dose calculated by using weighting factors for sensitive internal organs is closer to 500-600 mrem. Scintimammography obtained FDA approval in 1997.

Early studies of ${ }^{201} \mathrm{Tl}$ [35, 36] and ${ }^{99 m}$ Tc-sestamibi [22, 37, 38, 39, 40, 41] have shown that these agents are frequently taken up by breast cancers, and to a lesser extent, by other mammographically detected abnormalities. ${ }^{99 m}$ Tc-sestamibi 
(Miraluma $^{T M}$, DuPont Merck Pharmaceutical Company, North Billerica, MA), however, is currently the most promising tumor-avid tracer for scintimammography. It is a lipophilic cation originally developed as a cardiac perfusion agent and approved in the United States in December, 1990 for clinical use in the detection of coronary artery disease. Its chemical structure is given in Figure 2.4. The exact mechanism for cellular uptake of sestamibi by cancer cells is still uncertain, but sestamibi is known to be a P-glycoprotein transport substrate and this could be the tracer's means of uptake [44]. Sestamibi appears to accumulate in cells in relation both to perfusion and to the negative plasma and mitochondrial membrane potentials (which exert an electrostatic force on the positively-charged sestamibi) [42, 43]. The accumulation of sestamibi in chick myocardial cells has been shown to depend heavily on the retention of the radionuclide by mitochondria $[45,46]$. Further, the mitochondrial retention of sestamibi does not appear to be organ-specific - in human carcinoma cell cultures over $90 \%$ of the activity is concentrated in the mitochondria [47]. Studies suggest that the tumor-to-breast tissue uptake ratio in patient studies varies significantly but can be expected to be approximately $4[48,49,50]$. This contrast is substantially higher than that of X-ray mammography (where tumor tissue may only be $5 \%$ denser than normal tissue) and represents an important strength of scintimammography. Although ${ }^{99 m}$ Tc-sestamibi is presently the ligand most frequently used for scintimammography, other tracers such as ${ }^{201} \mathrm{Tl},{ }^{99 m} \mathrm{Tc}-\mathrm{MDP},{ }^{99 m} \mathrm{Tc}$-tetrofosmin, radiolabeled antibodies, radiolabeled estrogen receptor ligands, radiolabeled somatostatin compounds, and radiolabeled chemotherapeutic agents are currently under active investigation $[10,35,51,52,53]$.

A number of investigators have demonstrated sensitivities of $83-94 \%$ and specificities of $88-93 \%$ for scintimammography imaging of suspected breast lesions using sestamibi and conventional Anger cameras [54, 55, 56, 57]. Evidence further suggests that this modality performs equally as well when imaging radiographically dense and/or glandular breasts [58]. While all of this is indeed cause for optimism, caution must be exercised with scintimammography because the sensitivity can drop 


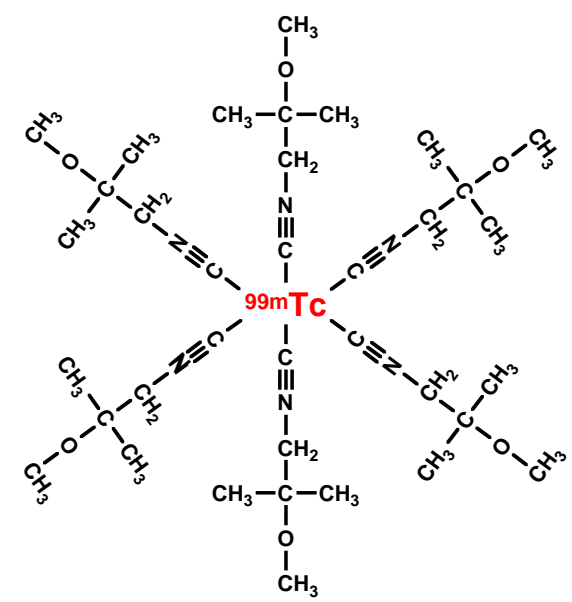

Figure 2.4: Chemical structure of ${ }^{99 m}$ Tc-sestamibi, the most commonly used scintimammography radionuclide tracer. It has an overall charge of +1 , making it susceptible to electrostatic forces.

to as low as $62 \%$ in patient populations with predominantly non-palpable lesions $[42,59]$ and sensitivity for tumors less than $1 \mathrm{~cm}$ in diameter is often poor [60,61]. Finally, scintimammography has also shown some promise in evaluating the axillary lymph nodes, demonstrating sensitivities of $64-82 \%$ and specificities of $78-90 \%$ [33, $54,60,62]$.

It is believed that the number of false negatives (i.e., missed tumors) could be reduced if the limitations of contemporary gamma cameras were overcome. For example, reference [55] discovered that three of the four false negatives which occurred during the study involved tumors in the medial part of the breast in a volume that was out of close camera contact. Detection of breast lesions in the internal quadrants is especially important because these tumors may disseminate towards the internal mammary chain even when no axillary node is invaded [63]. This problem could be solved by compact, thin gamma cameras that eliminate the dead space and large size which are typical of traditional gamma cameras and which limit access to the breasts and axillary nodes. Easy access to all nodes and potential breast lesion sites 
will improve image quality and can be expected to improve the diagnostic accuracy of scintimammography.

While researchers agree that scintimammography is unlikely to replace Xray mammography as a mass screening tool, most believe that many patients can benefit from it. Specific applications are presented in Sections 1.3.2 and 2.4.5, and more may potentially be added as this modality continues to develop and evolve. In brief, scintimammography is attractive when X-ray mammography is impaired, namely in women with dense or scarred breasts. Scintimammography may also be used to check for multicentric disease, to evaluate the effectiveness of chemotherapy as treatment progresses, and, if its sensitivity can be improved, to noninvasively evaluate axillary lymph node involvement. Finally, any tumors visible only to scintimammography scans will require scintimammography-guided biopsy techniques.

\subsubsection{Positron Emission Mammography}

Preliminary studies suggest that positron emission tomography (PET)or positron emission mammography (PEM) as it is known when applied specifically to the breast - using the tracer ${ }^{18} \mathrm{~F}$-fluorodeoxyglucose (FDG) can potentially provide excellent sensitivity $(\sim 90 \%)$ and specificity ( $\sim 90 \%)$ for both malignant breast tumors and axillary node involvement [64, 65, 66, 67]. Like scintimammography, PEM is an adjunct to X-ray mammography and potentially fulfills many of the same roles as scintimammography (see Section 2.4.5). PEM is attractive in comparison to scintimammography because it allows quantitation studies, provides superior spatial resolution, and can make use of more biologically interesting tracers by exploiting the positron emitters ${ }^{11} \mathrm{C},{ }^{13} \mathrm{~N}$, and ${ }^{15} \mathrm{O}$. While PEM typically focuses on producing high quality images of only a small section of the body (usually the breast or axillary nodes), full body PET can be used to identify the presence or absence of distant metastatic tumors in a patient who is already known to have a primary lesion in the breast, thereby aiding in diagnosis and treatment selection. 
Despite these strengths, however, the great expense of both full body PET imaging systems and tracers labeled with a positron emitter (which require a cyclotron to manufacture) have prevented PEM imaging from establishing a significant clinical presence. Recent PET research, however, has focused on developing smaller, breast-specific PET cameras that will require substantially less hardware and therefore be much less expensive $[68,69]$. One such device is depicted in Figure 2.5. Comparing this modality to scintimammography, single photon cameras are substantially less expensive than their PET counterparts - traditional Anger cameras are less expensive than full body PET scanners and compact CsI(Tl)/Si photodiode cameras should prove less expensive than advanced PEM systems like the one shown in Figure 2.5. Further, single photon tracer compounds using ${ }^{99 m}$ Tc can easily be supplied with a conventional ${ }^{99} \mathrm{Mo}-{ }^{99 m} \mathrm{Tc}$ generator and a synthesis kit, making them substantially less expensive than their cyclotron-dependent PEM equivalents. Whether the advantages of PEM imaging will outweigh the additional expense compared to scintimammography remains to be seen.

\subsubsection{Ultrasound}

Ultrasound is based on the reflection of acoustic energy at boundaries between tissues with different acoustic impedances. This allows for the localization of certain suspicious structures within the breast as well as the ability to differentiate between certain types of tissue. In the present clinical setting sonography is the most important imaging modality next to X-ray mammography because it can distinguish between cysts and solid masses with accuracy approaching 100\%, thereby reducing the number of unnecessary biopsies $[9,70,71]$. Sonography, however, has limited ability to differentiate small lesions from fat lobules, demonstrates examiner-dependent accuracy, and demands substantial physician time [9]. This modality is thus generally limited to the roles of evaluating suspected cysts and guiding biopsy. The role of ultrasound may potentially expand, however, with such developments as color 


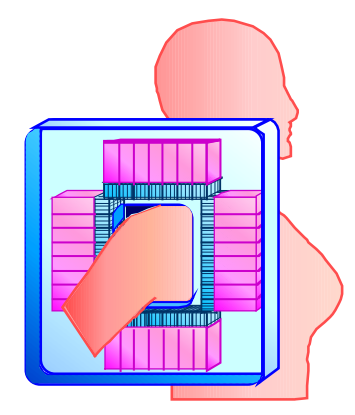

Figure 2.5: Positron Emission Mammography (PEM) camera currently under development using some of the same technology as the compact, discrete scintillation camera described in this thesis [68]. Breast imaging following injection of a positron tracer (most commonly FDG) is accomplished by placing the breast within the rectangular arrangement of detector modules and compressing gently. As is shown above, axillary node imaging is possible if the patient's arm is pushed far enough through the camera that the shoulder and armpit come into the camera's field of view.

Doppler, wherein color is used to encode blood velocity or volume [72, 73, 74, 75]. Malignant growths tend to develop more vascularization than either healthy tissue or benign tumors, hence cancer may be identifiable with color Doppler ultrasound by detecting abnormally high blood vessel density or peak blood flow velocity.

\subsubsection{Contrast-Enhanced MRI}

MRI operates by measuring the response of anatomical structures (and, if it is used, contrast agent) to applied magnetic fields. By injecting a paramagnetic contrast agent such as gadolinium (Gd) into the vascular system, the details of blood flow and vascularization can be better observed. Contrast-enhanced MRI has demonstrated a high sensitivity to small (less than $1 \mathrm{~cm}$ ) lesions in the breast, a situation in which the sensitivity of conventional scintimammography imaging is low. However, the specificity of MRI scans of suspicious structures is as low as 30- 
$70 \%$ because fibroadenomas, proliferative benign disorders, areas of inflammation, and active glandular tissue can all show enhancement $[9,76,77,78,79,80]$. The specificity improves slightly when dynamic techniques are used to measure the rate of contrast agent uptake during the initial minutes after contrast injection [81], but this specificity increase comes at the expense of spatial resolution, coverage of the breast, and/or longer study times [82]. The axillary nodes tend to be poorly evaluated with MRI mainly because contrast enhancement occurs for both normal and pathological nodes [81], although recent work with dynamic techniques does show progress in this area $[83]$.

In general MRI is currently restricted to cases in which X-ray mammography and biopsy do not provide adequate information-much as are scintimammography and PEM — and, in some cases, to guiding biopsy. While MRI does potentially fill similar niche roles as scintimammography and PEM, there are some notable differences. MRI, for instance, has been shown to be quite effective in imaging breasts with silicone implants [81] but is contraindicated when the patient is less than 35 or shows signs of breast inflammation [9].

\subsubsection{Summary of the Roles of Different Imaging Modalities}

The appropriate procedures for detecting, diagnosing, and treating breast cancer are quite complicated and depend heavily on patient history, the physician's preferences, the available imaging resources, and patient decision-making. While there may be significant variability from patient to patient, Figure 2.6 displays the fundamental progression of procedures that are usually implemented. I have generated this diagram based on my study of the available literature (beginning with reference [9] and including many of the other sources cited in this chapter) both on breast cancer screening and on clinical trials examining a wide range of imaging and diagnostic techniques. Emphasized in Figure 2.6 are the roles that X-ray mammography, ultrasound, scintimammography, PEM, and contrast-enhanced MRI 
currently do, and in the future potentially will, play. Of prime concern to the work presented in this thesis, obviously, are the possible uses of scintimammography, for these are the procedures wherein compact, discrete gamma cameras may improve breast cancer detection and diagnosis. Compact gamma cameras may contribute to scintimammography not only by offering superior imaging capabilities in these roles, but also in a research capacity by allowing for better evaluation of the efficacy of new radiopharmaceutical tracer agents. 


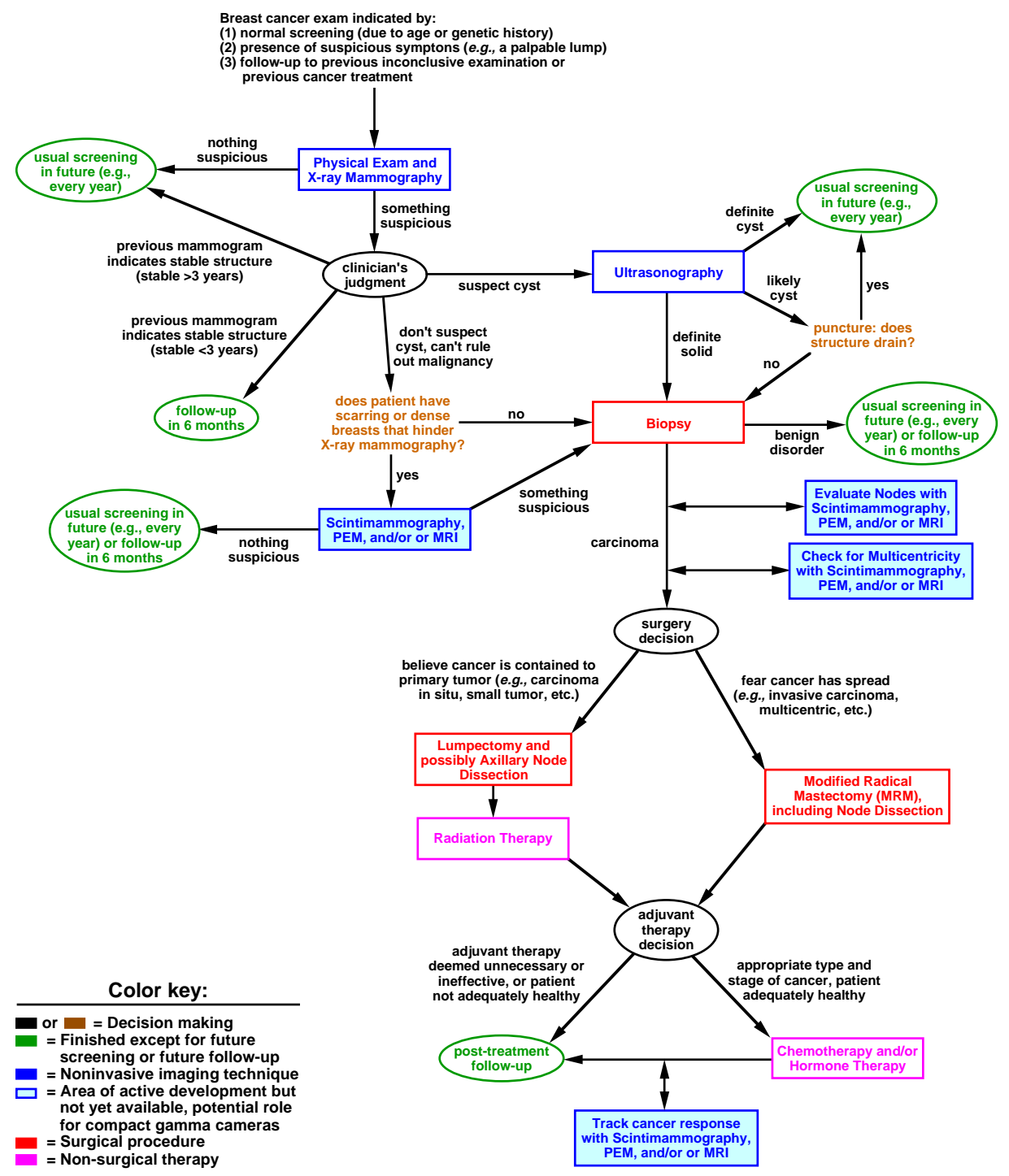

Figure 2.6: Basic decision-making tree summarizing the procedures used to detect, diagnose, and treat breast cancer. The boxes denoting scintimammography, PEM, and contrast-enhanced MRI procedures are not yet well established but may see increased use in those roles as relevant techniques and instrumentation are further improved. Not shown in the tree are the possible use of neoadjuvant treatment, the possible use of chemotherapy as the primary treatment if the cancer is known to have metastasized, and the possible use of other forms of mastectomy. 


\section{Chapter 3}

\section{Fundamentals of Single Photon Gamma Cameras}

Radioisotope cameras are in a state of development, and improvements can be expected...The uses of radioisotope cameras will undoubtedly become more important in medical diagnosis and research in the near future.

—Hal O. Anger, Survey of Radioisotope Cameras, 1966

\subsection{Anger Scintillation Cameras}

The first instrumentation that could generate images of radionuclide distributions appeared in the late 1940s, but it was simplistic and of limited use. The 1950s saw the advent of Benedict Cassen's rectilinear scanner, wherein a single radiation detector element could be mechanically moved ("scanned") across the area of interest and, given enough time, generate an image from the data accumulated at different locations. Rectilinear scanners have been greatly improved over the years and still find some use in nuclear medicine today, but fill relatively small roles. A more decisive advance in nuclear medicine occurred in 1953 when Hal Anger first described 
a system capable of observing radioactivity at all points in an image simultaneously. This first configuration used a relatively thin block of $\mathrm{NaI}(\mathrm{Tl})$ scintillator to cover the target area and a sheet of X-ray film to record the resulting scintillation events. Unfortunately, this detector was so inefficient that prohibitively long imaging times and/or high levels of administered radionuclide were required.

The real breakthrough came in 1958 when Hal Anger invented his scintillation camera (also known as a gamma camera or Anger camera), a device that has dominated radionuclide imaging ever since. This camera design uses a single block of $\mathrm{NaI}(\mathrm{Tl})$ scintillator large enough to cover the desired imaging area and thick enough to efficiently stop gamma rays. The major improvement over the previous incarnation, however, is the use of an array of photomultiplier tubes (PMTs) to detect the scintillation light produced by the $\mathrm{NaI}(\mathrm{Tl})$. This array of photosensitive devices provides reasonably efficient detection of the scintillation light, good spatial localization by means of Anger logic, and excellent pulse height (i.e., energy) resolution. Over the years the Anger scintillation camera has evolved and undergone significant improvements, but the basic concept has largely remained the same. No other nuclear medicine imaging system has yet to truly challenge the Anger camera for its combination of image quality, affordability, and ease of use in a hospital setting, but recent technological advances may change that in the future.

\subsubsection{Basic Principles}

A scintillation camera functions by converting individual gamma rays into thousands of visible light photons, detecting those photons with photosensitive elements that generate electric signals, and finally integrating the information carried by the electric signals into a coherent image. The basic components of an Anger scintillation camera include a collimator, a large $\mathrm{NaI}(\mathrm{Tl})$ scintillator crystal block, an array of PMTs (optically coupled to the scintillator), electronics which read out the electrical pulses generated by the PMTs, and a computer/image display system 
for presenting the data. Additionally, the $\mathrm{NaI}(\mathrm{Tl})$ is shielded from stray radiation with lead and hermetically encased to protect it from moisture in the air (because $\mathrm{NaI}(\mathrm{Tl})$ is very hygroscopic). The $\mathrm{NaI}(\mathrm{Tl})$ crystal block is typically cylindrical with a diameter of about 30-50 cm (though rectangular designs covering comparable area also exist) and a depth of about $1.25 \mathrm{~cm}$. For cameras dedicated to imaging lowenergy sources such as ${ }^{201} \mathrm{Tl}$ or ${ }^{99 m} \mathrm{Tc}$, a lesser $\mathrm{NaI}(\mathrm{Tl})$ thickness of only $6-8 \mathrm{~mm}$ is employed. The slight decrease in radiation detection efficiency is justified by the improved energy and spatial resolutions resulting from better light collection by the PMTs [13]. Finally, the readout electronics consist of two subsystems. The first is the Anger logic circuitry, which estimates the 2-D location of the observed radiation event. The second is the pulse height discriminator, which estimates the energy of the gamma ray based on the PMT pulse amplitudes and allows only events within the appropriate energy range to be incorporated into the image (energy discrimination). This rejects scattered gamma rays containing little useful information and improves the noise quality of the resulting images. The basic components of an Anger scintillation camera are summarized in Figure 3.1.

PMTs are critical to the operation and performance of Anger scintillation cameras but present very significant requirements in terms of volume, component cost, and voltage supply. These devices are typically about $5-8 \mathrm{~cm}$ in diameter by about $12 \mathrm{~cm}$ in length, cost around $\$ 150$ to $\$ 200$ each, and operate off of a $>1 \mathrm{kV}$ supply. Higher quality cameras tend to use smaller PMTs, increasing accuracy but necessitating a greater number of devices and therefore increasing cost. PMT quantum efficiency for the $415 \mathrm{~nm}$ scintillation photons produced by $\mathrm{NaI}(\mathrm{Tl})$ is only about $20-25 \%$ (i.e., only about 1 in 4 or 5 photons striking the PMT face is actually detected), contributing to the counting statistic limitations discussed below.

The readout electronics (both the Anger logic and the pulse height analyzer) rely upon the fact that the height of an output pulse from a given PMT is linearly proportional to the number of scintillation photons striking its face. As a result, a reasonable measure for the total number of scintillation photons produced by a 


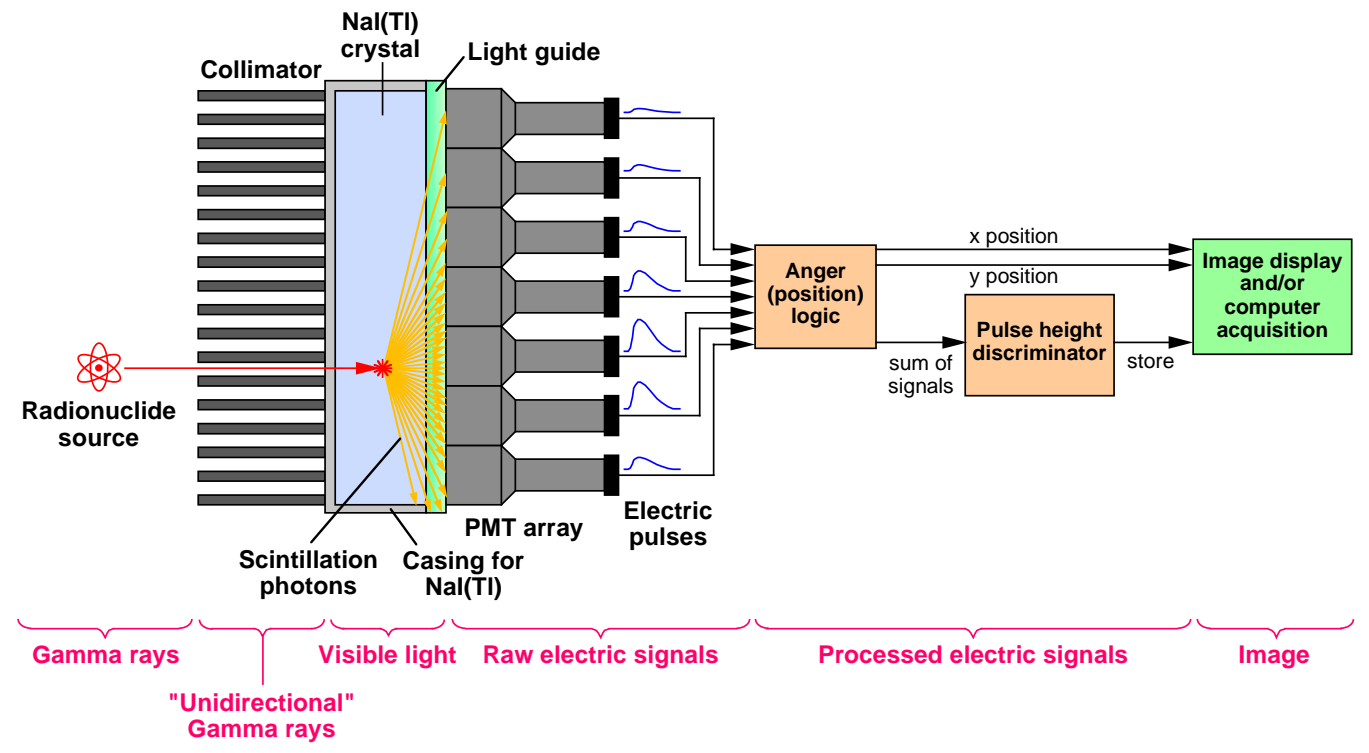

Figure 3.1: Basic components of the Anger scintillation camera. A parallel hole collimator is depicted, but a pinhole, converging, or diverging collimator could also be used. Not shown are scintillation photons originally travelling away from the PMTs (the emission of scintillation photons is isotropic), which may reflect off a crystal wall back toward the PMTs and be detected. 
gamma interaction is given by the sum of the pulse heights of all the PMT signals. Further, because the number of scintillation photons generated by the crystal is proportional to the energy of the incident gamma ray, the energy of observed gamma rays can be estimated as:

$$
\text { gamma energy } \left.\propto \sum_{i=1}^{n} \text { (pulse height from } P M T_{i}\right)
$$

where $n$ is the total number of PMTs in the array. Calibration can easily be performed using a source of known energy in the absence of scatter. The calibrated output from Equation 3.1 can thus be used for purposes of energy discrimination, as discussed in Section 1.2.5.

The number of photons impinging upon each PMT depends heavily upon the location of the gamma ray interaction, and Anger logic exploits this dependency by weighting each PMT based upon the height of its output pulse. Figure 3.2 shows an array of 37 PMTs used to read out the scintillation light from a large $\mathrm{NaI}(\mathrm{Tl})$ crystal. It is straightforward to assign the center of each PMT an $(x, y)$ coordinate and then compute the location of interaction as the centroid of the PMT signals:

$$
x_{\text {interaction }}=\frac{\sum_{i=1}^{n} x_{i}\left(\text { pulse height from } P M T_{i}\right)}{\sum_{i=1}^{n}\left(\text { pulse height from } P M T_{i}\right)}
$$

where $x_{i}$ is the $x$ coordinate of a given PMT and $n$ is the total number of PMTs. The $y$ coordinate of the interaction site is also computed using Equation 3.2, but naturally with the appropriate substitutions of $y$ for $x$.

Measurement error in scintillation cameras has three predominant sources: (1) light collection inhomogeneity, (2) electronic noise, and (3) limited counting statistics. Light collection inhomogeneity refers to the fact that the total number of visible light photons observed by the photodetector system (in the case of an Anger camera, the array of PMTs) varies depending on the site of gamma ray interaction. The farther the interaction is away from the face of the PMTs, for instance, the 


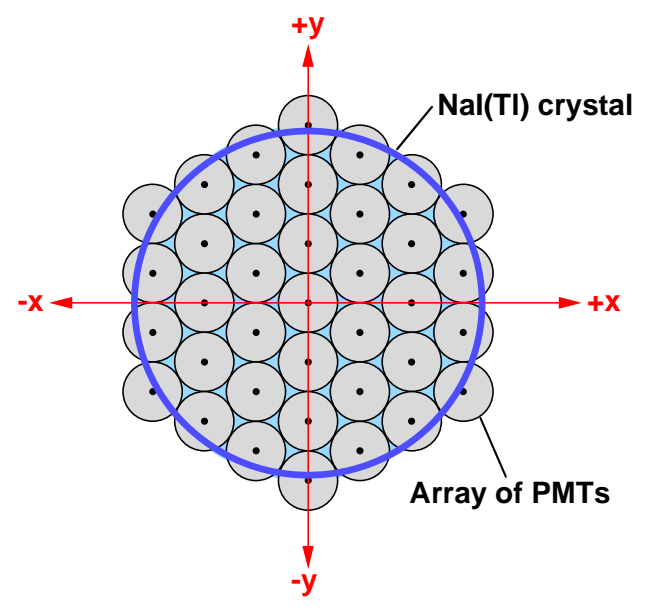

Figure 3.2: An example array of 37 PMTs used in an Anger scintillation camera. Circular PMTs are shown, but square or hexagonal PMTs can be used to improve packing. The output pulse height from each PMT is used in conjunction with the corresponding $(x, y)$ position of the device to determine the location of the gamma ray interaction (see Equation 3.2).

fewer scintillation photons tend to be detected. Location-dependent differences in light collection can also occur because of crystal defects, light guide aberrations, or variations in photodetector quantum efficiency. All of this contributes to error in energy resolution (as per Equation 3.1) because the total number of observed scintillation photons varies independently of gamma ray energy. Inhomogeneity also introduces variations into the terms in Equation 3.2, resulting in increased spatial localization error. Finally, near the edge of the scintillator crystal in an Anger camera, the reflection of photons off the side of the crystal is a significant source of error, worsening energy resolution and greatly skewing spatial localization.

Electronic noise increases the error in both the energy and location measurements by adding random fluctuations to the information-carrying electric signals. In the case of Anger cameras, however, this noise component is negligible because the PMT gain is large enough (around $10^{6}$ ) that the resulting signals are immune to 
small noise variations.

Finally, limited counting statistics represent a significant source of error, especially in Anger logic. All of the pulse height terms in Equation 3.2 suffer Poisson fluctuations as per Equation 1.6, error which propagates into the final $x$ and $y$ coordinates. Counting statistics also contribute to error in energy resolution, but the effect is less pronounced because all of the PMT signals are summed together in Equation 3.1, resulting in a larger signal, $N$, and hence smaller relative noise, $\sigma / N$.

Another potential source of error in scintillation cameras is Compton scattering within the detector crystal. Such interactions allow the incident gamma ray to deposit energy (and generate scintillation photons) at multiple locations, possibly producing an inaccurate location measurement. However, for single photon energies of interest (e.g., the $140 \mathrm{keV}$ emissions from ${ }^{99 m} \mathrm{Tc}$ ) the photofraction is large enough that the Compton error is negligibly small [13]. For perspective, fewer than $10 \%$ of highly energetic $662 \mathrm{keV}$ gammas (which therefore have a lower photofraction) are misplaced by more than $2.5 \mathrm{~mm}$ due to Compton scattering within a $\mathrm{NaI}(\mathrm{Tl})$ crystal $1 / 8$ inch thick [84].

All told, large Anger scintillation cameras typically achieve intrinsic spatial resolution of about $3.5 \mathrm{~mm}$ fwhm and energy resolution of about $9 \%$ fwhm, proving themselves extremely valuable for both static and dynamic single photon studies as well as for both planar and SPECT imaging. These cameras have been critical to recent scintimammography clinical trials $[54,55,56,57]$. However, Anger cameras are not optimized for the task of breast or axillary node imaging because of their significant size and problematic dead space near the periphery of the camera. The size stems from the bulky nature of the $\mathrm{NaI}(\mathrm{Tl})$ hermetic assembly, the lead shielding, and the cumbersome PMT array, generally resulting in a camera about the size of a car tire and making access to the breast and axillae somewhat difficult as shown in Figure 3.3. The camera cannot be pressed tightly against the tissue to be imaged, hence the extra imaging distance worsens collimator spatial resolution and thereby reduces image quality. At an imaging distance of $10 \mathrm{~cm}$ (which is very possible in 

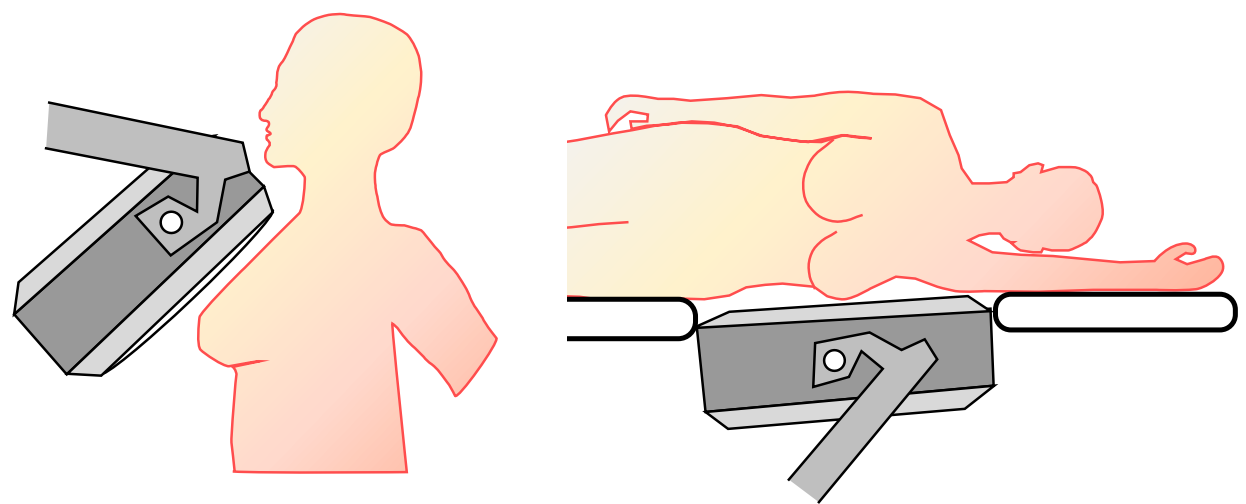

Figure 3.3: An Anger scintillation camera applied to scintimammography. The large size of the device makes access to the breasts and axillae difficult, hence the camera is not optimal for the task of scintimammography.

this scenario), the spatial resolution of an Anger camera employing a general purpose collimator (a fairly high resolution selection for this application) is about $1 \mathrm{~cm} \mathrm{fwhm}$. If greater sensitivity is required, the spatial resolution obviously suffers even further. Detecting small tumors on the order of $1 \mathrm{~cm}$ or smaller in size is therefore quite challenging with this non-optimal imaging system.

Dead space (where image data are not collected) exists around the periphery of an Anger camera because: (1) both the radiation shielding and the hermetically sealed assembly for the $\mathrm{NaI}(\mathrm{Tl})$ take up space, and (2) toward the edges of the $\mathrm{NaI}(\mathrm{Tl})$ crystal the Anger logic for position localization begins to fail. Equation 3.2 depends on having PMTs on all sides of the site of interaction, something that is obviously not true near the edge of the $\mathrm{NaI}(\mathrm{Tl})$ crystal. Worse, because PMTs packed for maximum compactness (and hence maximum coverage of the $\mathrm{NaI}(\mathrm{Tl})$ crystal) do not form a perfect circle, some of the PMTs will extend beyond the border of the crystal, as shown in Figure 3.2. The end result is that extra physical space (usually several 


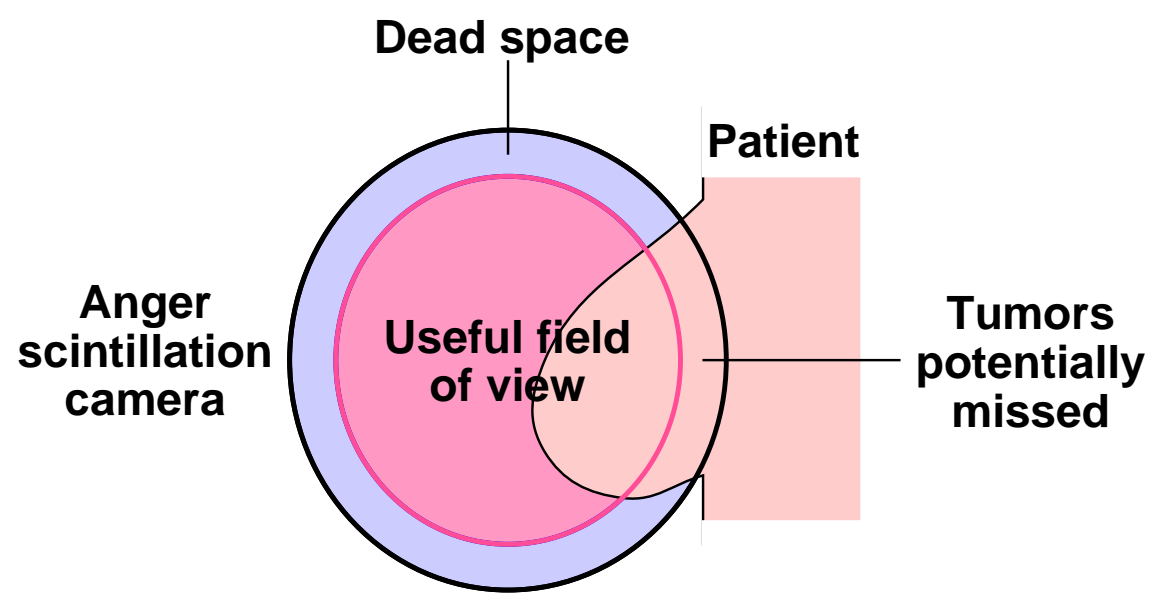

Figure 3.4: The dead space at the periphery of an Anger camera impedes scintimammography imaging of lesions. When taking a medial view of the breast (center of the body looking outward), tumors near the chest wall may not be in the active field of view of the camera and may therefore go undetected.

inches) exists where no useful imaging occurs. Figure 3.4 shows the deleterious effect this dead space can have on scintimammography. In the case of a medial view of the breast, the dead space prevents tissue near the chest wall from being imaged and may potentially allow tumors there to go undetected. For lateral views of the breast, on the other hand, the FOV may be moved to give better coverage, but only at the expense of greater imaging distance and reduced image quality.

\subsubsection{NEMA Performance Characteristics}

Obviously the imaging characteristics of Anger cameras have certain inherent limitations. It is important to understand and quantify these characteristics in order to evaluate their impact on image quality, allow meaningful comparisons between different single photon imaging systems, and generally aid in the development of more advanced imaging instrumentation. For these purposes the National Electrical Manufacturer's Association (NEMA) has developed a uniform set of criteria 
for the measurement and reporting of scintillation camera performance [85]. As was discussed in Section 1.2.2, the collimator is almost always the limiting factor for both system spatial resolution and system sensitivity. It is not the only limiting factor, however, nor is it relevant to other performance metrics such as energy resolution. The NEMA standards, outlined below, are concerned not only with system performance but also with intrinsic (i.e., without a collimator) camera characteristics. Except where mentioned otherwise, the preferred radiation source is ${ }^{99 m} \mathrm{Tc}$.

Intrinsic spatial resolution is a measure of the width of the detector response to an ideal line source (approximated using a lead mask). This is reported as both fwhm and fwtm (full width at tenth max) values for count rates of both $\leq 20 \mathrm{kHz}$ and $75 \mathrm{kHz}$ in the $\pm 10 \%$ energy window (126 to $154 \mathrm{keV}$ for ${ }^{99 m} \mathrm{Tc}$ ). The former demonstrates the spatial resolution under relatively ideal conditions, while the latter reveals any performance degradation that may occur at higher count rates. Intrinsic spatial resolution is affected by the thickness of the $\mathrm{NaI}(\mathrm{Tl})$ crystal, the size of the PMTs, and, as discussed in Section 3.1.1, counting statistics.

Intrinsic energy resolution is a straightforward measure of the camera's ability to distinguish between gamma rays of different energies, as discussed in Section 1.2.5. It is expressed as a percentage and is equal to the photopeak fwhm divided by the photopeak center energy. The primary reasons for limitations in Anger camera energy resolution are addressed in Section 3.1.1.

Intrinsic spatial linearity refers to the differences in the measured positions of incident gamma rays and the true locations of these interaction sites. Essentially it is spatial distortion in the resulting image. The linearity of a camera is measured using a lead mask to mimic line sources and is reported as the displacement of measured positions from a best fit line. Because arrays of smaller (and more numerous) PMTs tend to provide more accurate spatial localization than arrays of larger PMTs, smaller PMTs generally improve linearity. Linearity errors also result from light collection inhomogeneities, specifically variations in PMT sensitivity and defects in the $\mathrm{NaI}(\mathrm{Tl})$ or light guide. 
Intrinsic flood field uniformity is the regularity in the response of the camera, without a collimator, to a uniform, planar radiation source (approximated using a point source at a significant imaging distance). This study should reveal any undesirable "hot" or "cold" spots in images that occur because of instrumentation imperfections. Results are reported for both the useful field of view (UFOV) of the camera, as well as for the central field of view (CFOV) wherein the dimensions of the UFOV have all been scaled down by a factor of 0.75 . Values measured include the maximum pixel count deviation across the UFOV, CFOV, and a small range intended to approximate the size of a PMT. Studies are performed at both $\leq 20 \mathrm{kHz}$ and $75 \mathrm{kHz}$ in the $\pm 10 \%$ energy window. As with linearity errors, uniformity distortions typically result from variations in PMT sensitivity or defects in the scintillator crystal or light guide. The uniformity of the entire imaging system can also be affected by collimator irregularities.

Intrinsic count rate performance is a measure of how quickly the camera system can process event data, which is critical to determining how many gamma interactions will be entirely missed because the device was busy with a previous event. The dead time of an imaging system, $\tau$, is the time required to process a single gamma interaction and is due to the fact that the emission of scintillation light and the subsequent production of electronic pulses have finite durations. Should a second interaction occur during that time, $\tau$, the scintillation photons and electric signals from the new event will be superimposed with those from the previous one, creating distorted waveforms and missed information. There are two types of dead time pileup - paralyzable and non-paralyzable. In the non-paralyzable case, if a second event occurs during the dead time, $\tau$, that event is simply ignored and camera operation proceeds as normal after the original dead time has expired. The resulting detection event rate, $R_{\text {observed }}$ is given by:

$$
R_{\text {observed }}=\frac{R_{\text {true }}}{1+R_{\text {true }} \tau}
$$

where $R_{\text {true }}$ is the rate at which gamma rays interact in the detector system. 
Even worse than the situation expressed in Equation 3.3 is a paralyzable system wherein each interaction creates a dead time, $\tau$, even if that particular event occurred during another gamma ray's dead time (and was therefore not counted). As a result, in a long train of closely-spaced events only the first will be counted. In the paralyzable scenario the detected event rate, $R_{\text {observed }}$, is calculated as:

$$
R_{\text {observed }}=R_{\text {true }} e^{-R_{\text {true }} \tau}
$$

The impact of Equations 3.3 and 3.4 are depicted graphically in Figure 3.5. Anger scintillation cameras have a mixture of paralyzable and non-paralyzable dead times. The former is due to the finite fluorescence decay time of $\mathrm{NaI}(\mathrm{Tl})$ - the decay time for the emission of scintillation photons is about $0.23 \mu \mathrm{s}$. Multiple gamma rays interacting in quick succession can produce erroneous PMT signals because scintillation photons from both events are detected simultaneously. The non-paralyzable part of the camera system generally includes a lot of the signal processing electronics, including the pulse height analyzer.

NEMA standards require that two count rate values be measured - the observed count rate that results in a $20 \%$ count loss and the overall maximum count rate. These measurements are made both in air and with induced scatter. Typical maximum count rates for Anger cameras vary in the $100-500 \mathrm{kHz}$ range.

System spatial resolution is reported as fwhm and fwtm values - much like intrinsic spatial resolution - and is measured with a collimator located in front of the scintillator detector system. Capillary tubes less than $1 \mathrm{~mm}$ in diameter filled with a radionuclide represent ideal line sources and are imaged at distances of 50 and $100 \mathrm{~mm}$. The count rate is kept less than $20 \mathrm{kHz}$ in the $\pm 10 \%$ energy window and the measurements are made both in air and with induced scatter. Both the collimator and the intrinsic spatial resolution influence the result as per Equation 1.1, but the collimator is almost always the limiting factor.

System planar sensitivity is also measured with a collimator in place and at a count rate less than $20 \mathrm{kHz}$. It is reported as counts $/ \mathrm{min} / \mathrm{MBq}$ or counts $/ \mathrm{min} / \mu \mathrm{Ci}$ 


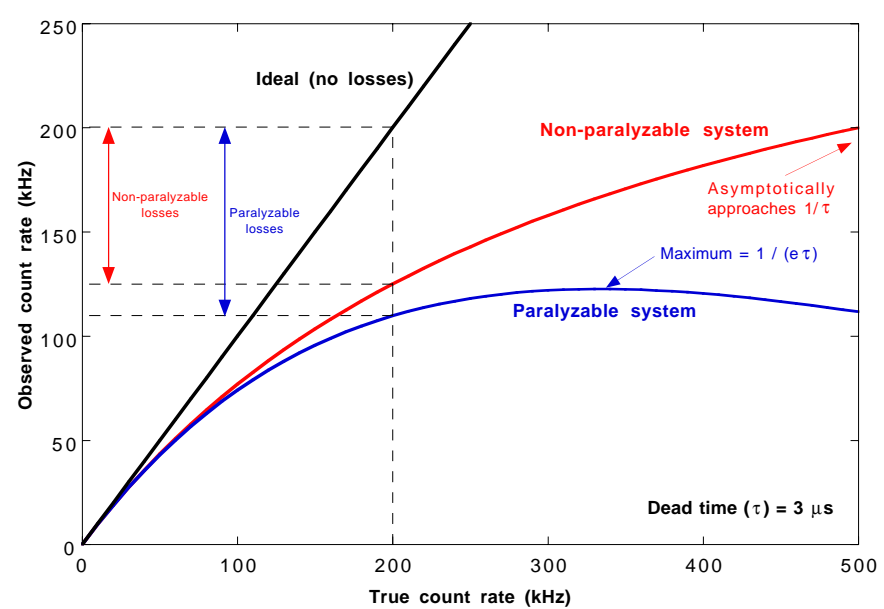

Figure 3.5: Dead time (the time required to process a detected radiation event) in a camera system limits the observed count rate. Count rate losses (i.e., missed events) increase with longer dead times, higher true count rates, and in systems that are paralyzable rather than non-paralyzable.

detected by the camera for a $100 \mathrm{~mm}$ diameter flat dish containing a radionuclide and placed in the camera CFOV at a distance of $100 \mathrm{~mm}$. Obviously the most important factor is the collimator design, as discussed in Section 1.2.2, but the detection efficiency of the scintillator crystal (determined primarily by its depth, as per Equation 1.7) also plays an integral role.

Multiple window spatial registration is a measure of the camera's ability to accurately position gamma rays of different energies using multiple energy acceptance windows. The source used for this measurement is ${ }^{67} \mathrm{Ga}$ and the photons to be imaged are either 93 and $300 \mathrm{keV}$ for two window systems, or 93, 184, and $300 \mathrm{keV}$ for three window systems. Count rates are kept below $10 \mathrm{kHz}$ in each $\pm 10 \%$ window, and results are reported as the $(x, y)$ displacement of the centroids of the images formed by gammas of different energies.

Detector shielding is important in preventing stray gamma rays from being detected by the camera and incorrectly incorporated into the image data. The 
effectiveness of the shielding is reported as a leakage value in counts/min/MBq or counts $/ \mathrm{min} / \mu \mathrm{Ci}$ and is measured by placing a source $100 \mathrm{~mm}$ from the detector housing. The source is to be moved around the sides and back of the camera to find the location of greatest leakage.

Modern scintillation cameras employ corrective circuitry to minimize many of the distortions discussed above, including spatial linearity, planar uniformity, and energy resolution. All three types of errors occur because of spatial variations in sensitivity to scintillation light which result from aberrations in the $\mathrm{NaI}(\mathrm{Tl})$ crystal, the light guide, or the PMT array. By calibrating the camera it is possible to correct the signals for these distortions and produce more accurate localization and energy pulse height values.

\subsection{Discrete Scintillator/Photodiode Cameras}

\subsubsection{Motivation for Compact Designs}

In recent years there has been growing interest in developing compact gamma cameras for certain applications - including scintimammography - where Anger scintillation cameras are suboptimal. As is addressed in Section 3.1.1, Anger cameras tend to be relatively large and bulky in size and demonstrate significant dead space around the periphery. Combined, these effects can make small organs relatively difficult to image with this technology - the large size necessitates greater imaging distances and therefore worse collimator resolution, while the dead space can result in the loss of critical image data. Figure 3.6, for instance, depicts scintimammography imaging with a compact gamma camera. Compared to the traditional Anger camera shown in Figure 3.3, a compact design allows shorter imaging distances, provides better access to the medial quadrants of the breasts, and permits configurations wherein multiple cameras take images from different views simultaneously. One final consideration is cost - some compact gamma camera technologies 
are likely to produce imaging systems that are significantly less expensive than Anger cameras and may therefore be more readily available to the general public. For the time being these new technologies tend to be targeted toward specific small-organ applications, as it is difficult to challenge traditional Anger scintillation cameras for generalized single photon imaging.

There are three major design approaches to the development of new compact gamma cameras:

- Discrete scintillator/photodiode cameras wherein the gamma rays interact in an array of optically-isolated scintillation crystals that are coupled 1-to-1 to an array of solid-state photodetectors;

- Solid-state cameras where the gamma rays interact directly with a pixellated solid-state detector;

- Position-sensitive photomultiplier tube (PSPMT) cameras where the gamma rays interact in one or more scintillation crystals which are subsequently read out by a single PSPMT.

Obviously each approach presents its own unique advantages and challenges. The discrete scintillator/photodiode design is the focus of this thesis and is discussed immediately below. The other two camera types are addressed in Sections 3.3 and 3.4 , respectively.

\subsubsection{CsI(Tl) Scintillator Coupled to Si PIN Photodiodes}

Perhaps the most straightforward way to modify Anger technology to a compact design is to replace the PMT array with an array of solid-state photodiodes. This not only greatly reduces the size of the photodetectors and potentially eliminates the dead space around the periphery, it reduces the voltage supply requirements from $>1 \mathrm{kV}$ to about $50 \mathrm{~V}$, permits operation in strong magnetic fields (critical for 


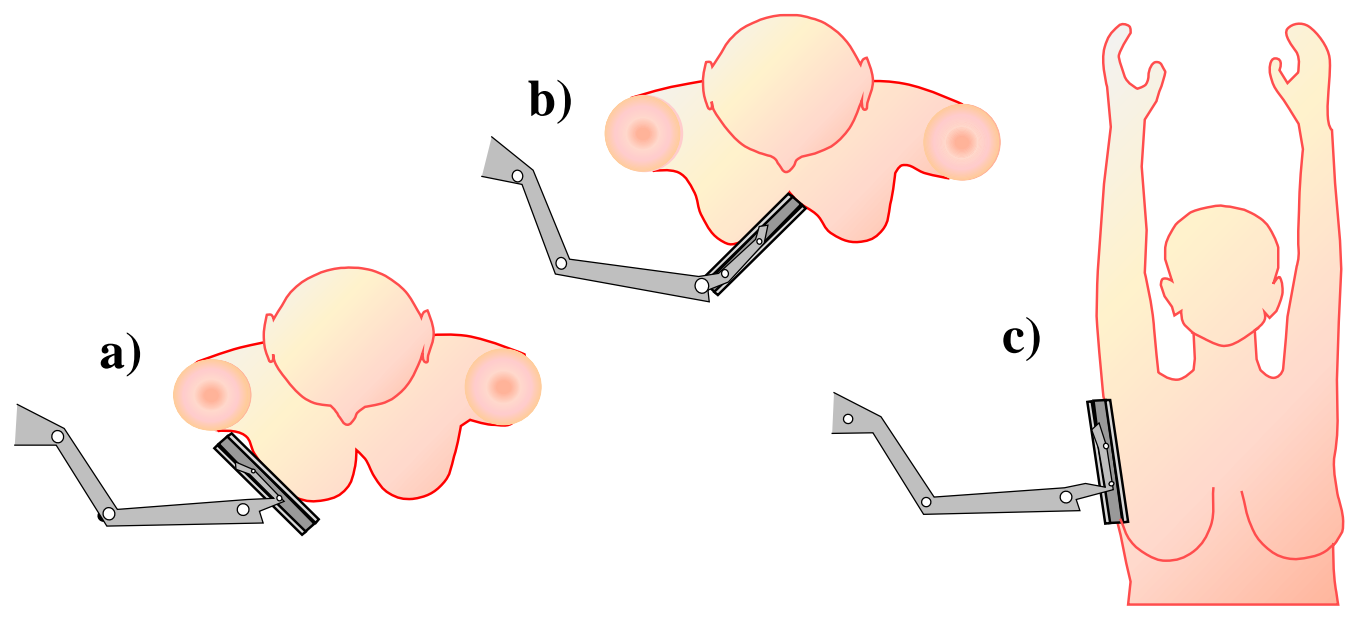

d)
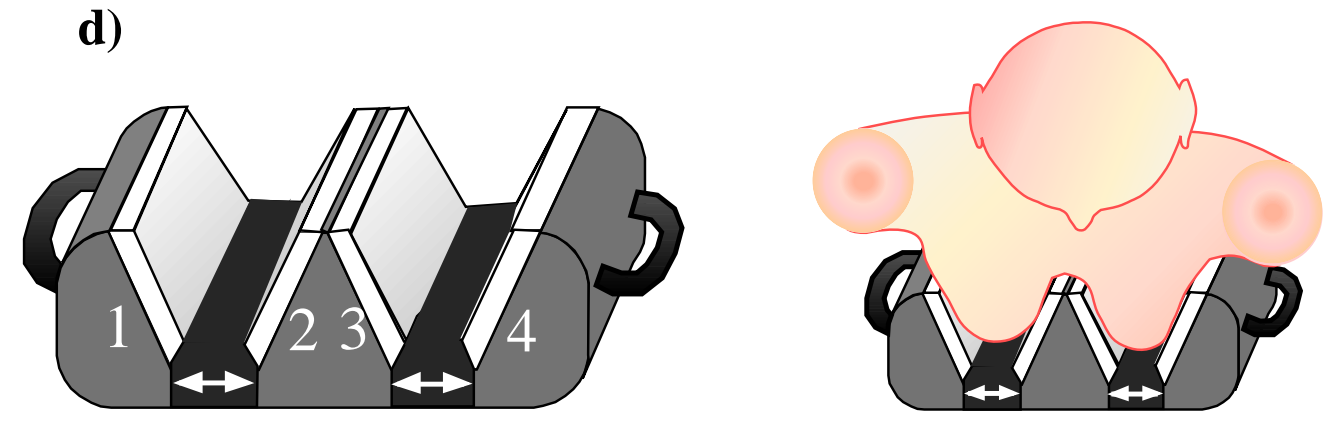

Figure 3.6: A compact gamma camera applied to scintimammography. The compact size allows the camera to: (a) get very close to the breast, (b) access the medial portion of the breast, (c) get very close to the axillary lymph nodes, and (d) be used in configurations that take multiple breast images simultaneously in order to maximize information collection and estimate tumor location in 3-D. By design this type of camera has very little dead space around the periphery. 


\begin{tabular}{|c|c|c|}
\hline Property & $\mathrm{NaI}(\mathrm{Tl})$ & $\mathrm{CsI}(\mathrm{Tl})$ \\
\hline Density $\left(\mathrm{g} / \mathrm{cm}^{3}\right)$ & 3.67 & 4.51 \\
$\lambda \max (\mathrm{nm})$ & 415 & 540 \\
Photons $/ 140 \mathrm{keV}$ & 5300 & 7000 \\
Decay time $(\mathrm{ns})$ & 230 & $800(67 \%), 3000(33 \%)$ \\
Attenuation length at $140 \mathrm{keV}(\mathrm{mm})$ & 4.01 & 2.76 \\
Hygroscopic? & Yes & Only slightly \\
\hline
\end{tabular}

Table 3.1: Comparison of $\mathrm{NaI}(\mathrm{Tl})$ and $\mathrm{CsI}(\mathrm{Tl})$ scintillator properties.

any applications that also use MRI), and potentially reduces the cost since solidstate photodetectors can be inexpensive. Additionally, the $\mathrm{NaI}(\mathrm{Tl})$ scintillator can be replaced with $\mathrm{CsI}(\mathrm{Tl})$, which is advantageous because $\mathrm{CsI}(\mathrm{Tl})$ has greater light output and is much less hygroscopic. The only significant drawback of $\mathrm{CsI}(\mathrm{Tl})$ is that its fluorescence decay time is substantially longer and results in longer dead times than $\mathrm{NaI}(\mathrm{Tl})$. This is usually not a serious problem, however, because of the low event rates that typically result in single photon studies (because of the inherently low geometric efficiency of the collimator). $\mathrm{NaI}(\mathrm{Tl})$, not $\mathrm{CsI}(\mathrm{Tl})$, is the scintillator of choice for PMT-based cameras only because PMT photocathodes generally have about 20-25\% quantum efficiency for $415 \mathrm{~nm} \mathrm{NaI(Tl)} \mathrm{scintillation} \mathrm{light,} \mathrm{but} \mathrm{an}$ unacceptably low quantum efficiency near $5 \%$ for $540 \mathrm{~nm} \mathrm{CsI(Tl)} \mathrm{scintillation} \mathrm{light.}$ Silicon photodiodes, on the other hand, have good quantum efficiency from 400 to $900 \mathrm{~nm}$. These two scintillators are summarized in Table 3.1.

Several readout schemes are possible using photodiodes, including simply using a solid block of $\mathrm{CsI}(\mathrm{Tl})$ and Anger logic with the array elements. This approach, however, is generally inferior to using optically-isolated $\mathrm{CsI}(\mathrm{Tl})$ crystals that are matched 1-to-1 to photodiode elements. The intrinsic spatial resolution of such a camera design is defined almost entirely by the pixel size, hence the use of small elements can provide much better than $3.5 \mathrm{~mm}$ fwhm intrinsic spatial resolution. 


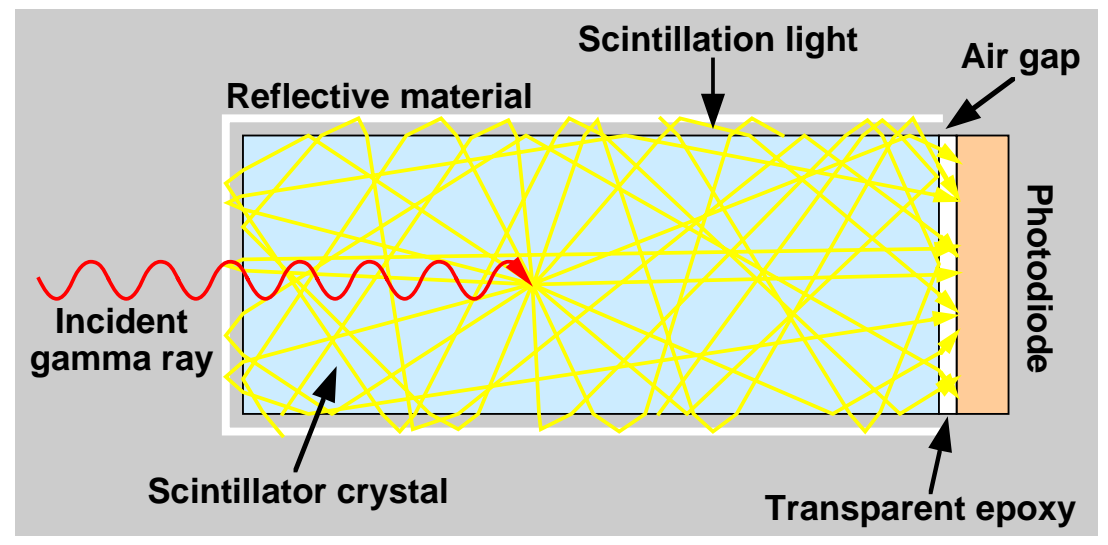

Figure 3.7: A single scintillator crystal coupled to a photodiode via transparent epoxy. As many scintillation photons as possible should be directed toward the photodiode in order to achieve the maximum signal for each interaction. Crystal surface treatment, the characteristics of the reflective surface covering five of the crystal's six sides, and the possible use of an air gap to achieve total internal reflection all affect how many photons reach the photodiode.

Further, because all scintillation photons from a given gamma interaction are directed toward a single photodiode, signal levels in that channel are larger in comparison to the size of the electronic noise. Individual crystals are longer than they are wide (e.g., $3 \times 3 \times 5 \mathrm{~mm}^{3}$ ), however, and successfully directing all scintillation photons to the photodiode requires careful treatment of the crystal sides and use of a high-quality reflective coating, as shown in Figure 3.7.

Despite the many advantages of a compact design using discrete $\mathrm{CsI}(\mathrm{Tl})$ crystals and solid-state photodiodes, it is only in the past several years that such cameras have begun to see clinical use. The primary reason for this is the large electronic noise that is associated with using photodiodes. Whereas PMTs have a large gain (typically $10^{6}$ ) and negligible electronic noise (providing a large signal-tonoise ratio), solid-state photodiodes have unity gain and significant noise in the form of leakage current, making them susceptible to electronic fluctuations. This obviously 
worsens energy resolution, making scatter rejection less effective and lowering image quality. One potential solution is cooling the photodiode arrays since it is possible to achieve an order of magnitude drop in leakage current per $20^{\circ} \mathrm{C}$ reduction in temperature. However, this requires a cooling system and introduces the additional complications of preventing problematic condensation and/or freezing of airborne moisture, as well as ensuring patient comfort with the camera temperature.

Technological advances have recently produced photodiodes with acceptably high signal-to-noise characteristics even at room temperature. The most promising of these are low noise, back-side illuminated silicon PIN (p-layer, intrinsic layer, nlayer) photodiodes [86], which are integral both to this thesis and to the first commercial discrete $\mathrm{CsI}(\mathrm{Tl}) /$ photodiode gamma camera (Model 2020tc Imager $^{T M}$, Digirad Corporation, San Diego, CA). These photodiodes have unity gain but demonstrate extremely low leakage current $\left(<5 \mathrm{pA} / \mathrm{mm}^{2}\right.$ at $50 \mathrm{~V}$ bias, compared to $\sim 30 \mathrm{pA} / \mathrm{mm}^{2}$ for typical silicon photodiodes) and therefore high signal-to-noise. Other photodiode technologies that have been under development and could potentially fill this same role include silicon drift photodiodes [87], avalanche photodiodes [88], and photodetectors made out of high band-gap materials such as $\mathrm{HgI}_{2}$ [89], $\mathrm{TlBr}(\mathrm{I})$ [90], or InI [91]. Of these competing technologies, the silicon PIN photodiode is the only photodetector that is presently well enough developed to be included in a reliable, cost effective, commercial camera.

The combination of CsI(Tl) scintillation crystals and silicon PIN photodiodes thus provides a favorable approach to low-cost compact gamma camera design (see Figure 1.4). Making the detector array large enough to view a significant section of the body is straightforward, $\mathrm{CsI}(\mathrm{Tl})$ proves a very effective scintillator at energies of interest (i.e., near $140 \mathrm{keV}$ ), low-noise silicon PIN photodiodes are available and are a good spectral match for $\mathrm{CsI}(\mathrm{Tl})$, both the scintillator and the photodiodes are inexpensive compared to pure solid-state detector materials like CdZnTe (see Section 3.3), and the entire camera is impressively compact. Prototype detector modules based on this approach are described in multiple references [92, 93, 94] and discussed 
further in Chapter 4.

Obviously CsI(Tl)/Si PIN photodiode detection technology does not change the fact that the collimator is a critical limiting factor in both spatial resolution and sensitivity. An added complication, however, is that for this type of camera the discrete pixels are typically square, yet the parallel holes of microcast lead collimators are most frequently hexagonal. This leads to a mismatch between the two and produces certain aliasing effects that can worsen image quality. Some recent research has simulated the performance of such configurations [93, 95, 96], while other work has examined the mathematical ramifications of this mismatch [97]. Generally hexagonal holes are acceptable, but new collimator fabrication technology can manufacture tungsten collimators with square holes which could be used to eliminate the mismatch altogether (Tecomet Collimation Technologies, Thermo Electron Tecomet, Wobrun, MA) [98]. Such collimators, however, are currently about an order of magnitude more expensive than microcast hexagonal hole lead collimators and have a $\sim 6 \mathrm{~mm}$ dead area around the periphery. Since these are not fundamental limitations of square hole collimators, the future may well these drawbacks alleviated. Collimator issues are treated extensively in Chapter 5.

\subsubsection{NEMA Performance Characteristics}

Multiple crystal scintillation cameras (e.g., CsI(Tl)/Si PIN photodiode cameras) are also covered by the NEMA standards. There are some small differences from the Anger camera measurement procedures discussed in Section 3.1.2, but by and large the standards are very similar. One difference is that intrinsic flood field uniformity is replaced by system flood field uniformity (i.e., the collimator is in place), since imperfect matching between collimator holes and detector pixels can easily cause pixel-to-pixel variations in sensitivity. 


\subsection{Solid-State CdZnTe Cameras}

The goal of any gamma camera is to convert incident gammas into electrical signals carrying image information. Scintillation cameras (Anger or otherwise) accomplish this by employing an intermediate step: gamma rays are converted to scintillation photons which are then converted into electric pulses. Each conversion step, however, results in a loss of energy and therefore lower signal levels. For instance, $\mathrm{NaI}(\mathrm{Tl})$ converts less than $15 \%$ of incident gamma ray energy into scintillation photons. The readout photodetectors experience losses as well because some of the scintillation photons are lost to absorption within the crystal or its reflective coating, and those that do reach the detectors are subject to the $<100 \%$ quantum efficiency of the element ( $\sim 20 \%$ for PMTs and up to $90 \%$ for photodiodes). Solid-state gamma cameras circumvent some of these losses because the gamma rays interact directly within semiconductor material. Instead of producing scintillation light the conversion process generates electron-hole pairs that can directly be detected as current. The primary advantage of this design is obvious: greater signal levels and therefore excellent energy resolution. Solid-state gamma cameras are pixellated and are therefore similar in form to discrete scintillator/photodiode cameras (Figure 1.4), but with those two detector materials replaced with a single semiconductor array with potentially sub-millimeter pixel size (providing excellent intrinsic spatial resolution). The advantages of compactness demonstrated in Figure 3.6 fully apply to solid-state cameras, making them strong contenders for scintimammography applications.

Solid-state materials must demonstrate a number of key characteristics in order to successfully perform as a gamma ray detector. They must have a large bandgap and therefore low leakage current in order to minimize electronic noise, they must have a high effective atomic number in order to provide adequate stopping power in a reasonable thickness of material, and they must allow efficient collection of the electron-hole pairs generated by a direct interaction. In addition to this, it must be possible to fabricate arrays of useful imaging size and thickness for a reasonable 
cost.

Germanium solid-state detectors cooled to liquid nitrogen temperatures have been under development for over 20 years [99, 100, 101] and have been used as one element of a Compton scatter coincidence camera [102]. Recent research into compound semiconductor materials, however, has introduced the possibility of room temperature operation with cadmium zinc telluride (also known as $\mathrm{Cd}_{1-x} \mathrm{Zn}_{x} \mathrm{Te}$, CdZnTe, or CZT), a high effective atomic number (about 50), large bandgap solidstate crystal $[103,104]$. A number of material related difficulties and limitations remain to be overcome, however. CdZnTe is expensive and difficult to fabricate in large arrays. Its behavior is temperature dependent, it exhibits substantial leakage current (limiting intrinsic energy resolution), and it suffers from incomplete charge collection due to carrier recombination and trapping (producing a low energy tail in the photopeak and therefore a loss of counts in the energy discrimination electronics). Semiconductor detector advances in "single-carrier charge collection," however, have allowed elimination of low energy tails and made CdZnTe cameras viable [105], resulting in several designs compatible with scintimammography [105, 106, 107].

The 2020tc Imager ${ }^{T M}$ (Digirad Corporation, San Diego, CA) described in references $[105,107]$ was the first commercial sold-state camera approved by the U.S. Food and Drug Administration for marketing as a general-purpose planar gamma camera, providing a pixel size of $3.1 \times 3.1 \mathrm{~mm}^{2}$, energy resolution of about $8 \%$ fwhm, a UFOV of $21.6 \times 21.6 \mathrm{~cm}^{2}$, compact design, and minimal dead area around the periphery. Despite this success, however, the costs associated with making large arrays of high-quality CdZnTe have encouraged Digirad Corporation to implement a new design for the 2020tc Imager $^{T M}$ based on the CsI(Tl)/Si PIN photodiode approach described in Section 3.2.2.

CdZnTe and other solid-state gamma cameras are not specifically covered by the NEMA standards described in [85], but the standards set forth for multicrystal scintillation cameras can generally be directly applied. 


\subsection{Position Sensitive Photomultiplier Tube Cameras}

A final approach to the design compact gamma cameras is to read out scintillator crystal(s) with a position sensitive photomultiplier tube (PSPMT) [108, 109]. PSPMTs are similar to PMTs in that they use a photocathode to convert incident visible light photons to electrons, then employ a high-voltage dynode structure to "multiply" those electrons into a large electric signal. As with PMTs, this potentially results in very high gain and minimal electronic noise. The position sensitivity of a PSPMT results from the use of an anode grid whose output signals not only indicate the intensity of the input light photons, but their position as well. A commonly used PSPMT in the medical imaging arena is the Hamamatsu R3292 (Hamamatsu Photonics K.K., Hamamatsu City, Japan), which is a $132 \mathrm{~mm}$ diameter device with a >100 mm active area and a total depth of $162 \mathrm{~mm}$. Scintillators used in conjunction with this and similar PSPMTs have included $\mathrm{NaI}(\mathrm{Tl}), \mathrm{CsI}(\mathrm{Tl}), \mathrm{CsI}(\mathrm{Na})$, and YAP. Designs have utilized one continuous crystal (much like in an Anger camera) or a discrete array of optically isolated crystals (much like discrete CsI(Tl)/Si PIN photodiode scintillator arrays). Various camera systems based on these approaches are described in references $[110,111,112,113,114,115]$, while devices designed specifically for scintimammography applications are addressed in [116, 117].

While PSPMT cameras have demonstrated certain success in medical imaging, the use of a PSPMT scintillator readout system presents a number of difficulties. First, while PSPMT cameras are definitely more compact than Anger cameras, the tube itself still retains a substantial depth of about $13 \mathrm{~cm}$. Further, while PSPMT cameras also undoubtedly have less dead area than Anger cameras, they still exhibit a dead zone at least $1.5 \mathrm{~cm}$ thick around the periphery of the PSPMT. The camera FOV is entirely dependent on the PSPMT dimensions, for unlike with discrete $\mathrm{CsI}(\mathrm{Tl}) / \mathrm{Si}$ PIN photodiode or CdZnTe cameras, there is no modular approach that makes construction of a camera of almost any size straightforward. As to performance issues, while PSPMTs can provide good spatial resolution, they are difficult 
to calibrate because they demonstrate geometric distortion and non-uniform gain that can vary with both time and count rate. Add to all of this the significant cost of PSPMTs, and PSPMT cameras do not presently appear to be an optimal design choice for scintimammography applications (though that does not preclude their use in countless other small organ or small animal imaging applications). For comparison with other camera technologies, the PSPMT scintimammography camera described in [116] provides $<2 \mathrm{~mm}$ intrinsic spatial resolution and $17 \%$ energy resolution.

PSPMT gamma cameras are not specifically covered by the NEMA standards described in [85], but it is generally reasonable to apply the standards set forth for the appropriate scintillator crystal configuration (i.e., those for either single crystal or multicrystal scintillation cameras). 


\section{Chapter 4}

\section{Prototype CsI(Tl)/Si PIN Photodiode Detectors}

It's all about signal and noise. Usually too much noise and not enough signal.

-Bill Moses

Naturally the design of any imaging device requires mastery of a substantial number of details and the proper management of engineering trade-offs. In this chapter I describe the construction and testing of a small prototype $\mathrm{CsI}(\mathrm{Tl}) / \mathrm{Si} \mathrm{PIN}$ photodiode imaging system [93] I used to gain insight into detector performance. The design of this small prototype was motivated by the need to characterize our camera technology — specifically a complete system including CsI(Tl) crystals, custom Si PIN photodiodes, and ASIC readout - and thereby contribute to final design decisions for the complete 64-pixel imaging modules which will form the building blocks of actual scintimammography cameras. With this ultimate goal of building an optimized scintimammography camera firmly in mind, it was therefore key to explore single pixel behavior, multiple pixel behavior, and means of maintaining compact design.

The prototype detector is a $3 \times 4$ element imaging array consisting of 
$3 \times 3 \times 5 \mathrm{~mm}^{3} \mathrm{CsI}(\mathrm{Tl})$ scintillator crystals coupled 1 -to-1 to $3 \times 3 \mathrm{~mm}^{2} \mathrm{PIN}$ silicon photodiodes designed for low leakage current ( $\sim 50 \mathrm{pA} /$ pixel at $50 \mathrm{~V}$ bias $)$ [86]. Photodiode output signals are amplified with a 16-channel custom IC $(<3 \mathrm{~mm}$ on a side) [119], after which a "Winner Take All" (WTA) custom IC (also $<3 \mathrm{~mm}$ on a side) [120] identifies the crystal of interaction based on relative signal amplitudes. The compact nature of this custom IC readout system simplifies the construction of larger imaging arrays, which is key to scaling up to the sizes need to build actual scintimammography devices. The target specifications include not only compact design, but also room temperature energy resolution of $10-15 \%$ fwhm and minimal pixel-to-pixel crosstalk.

The $3 \times 3 \mathrm{~mm}^{2}$ pixel size was chosen as a compromise. Smaller pixels provide improved intrinsic spatial resolution and present a smaller load, both in terms of capacitance and leakage current, to the readout ICs, which improves electronic noise and therefore intrinsic energy resolution. At the same time, however, smaller pixels increase the pixel density and therefore the number of readout channels, greatly complicating the implementation of the readout system. A pixel size of $3 \times 3 \mathrm{~mm}^{2}$ provides an intrinsic spatial resolution that is still less than that of most any collimator likely to be used in scintimammography (e.g., Table 7.1), a capacitive $(\sim 3 \mathrm{pF})$ and current ( $\sim 50 \mathrm{pA})$ load that the ASIC engineers felt would not overwhelm electronic noise performance, and a readout density that allows enough space for the required readout electronics.

Prototype measurements I have made and analyzed include signal levels, noise levels, optimal electronic shaping times, energy resolution, intrinsic spatial resolution, and system spatial resolution. Collimator design and custom IC readout design are addressed as pertains specifically to this prototype detector, but for complete coverage of these topics, see Chapters 5 and 6 , respectively. The final 64-pixel imaging modules which are the culmination of this work are described and characterized in Chapter 7. 


\subsection{Electronic Readout and Pulse Shaping}

Many of the fundamental design challenges inherent in a discrete $\mathrm{CsI}(\mathrm{Tl}) / \mathrm{Si}$ PIN photodiode configuration are mentioned in Section 3.2, but one that has yet to be addressed is the electronic readout hardware employed for each pixel. Proper design of the readout system is difficult because the electronics must meet two critical requirements: (1) low electronic noise in order to achieve good energy discrimination and (2) compactness in order to preserve many of the principal advantages of this camera configuration. The goal of low electronic noise motivates the use of a readout system composed of discrete components and amplifiers, but the space this would require when using 100s or potentially 1000s of pixels is completely prohibitive. Not only is it essentially impossible to achieve a compact design, but it becomes difficult to even route all of the individual photodiode signals to 100 s or 1000 s of discrete amplifiers without using problematically long leads that serve as antennas for transmitting and receiving noise. Probably the best answer to this dilemma is to use application specific integrated circuit (ASIC) readout of the photodiode signals, since this very effectively provides both short leads and compact design. Aside from the substantial expense of designing the ASIC, the major drawback of this approach is the increased electronic noise that often results from having to fabricate almost all the circuitry on a silicon wafer. With proper design, however, the noise can be limited to acceptable levels and a very effective readout scheme achieved. In fact, it is actually possible to obtain lower noise with an ASIC than with discrete components if the ability to custom tailor transistor sizes (e.g., match them to the detector) is used to greatest advantage. Before proceeding directly to the readout ASICs used in the prototype detector system, it is worthwhile to address some of the fundamental signal-to-noise concerns that govern their design and operation. 


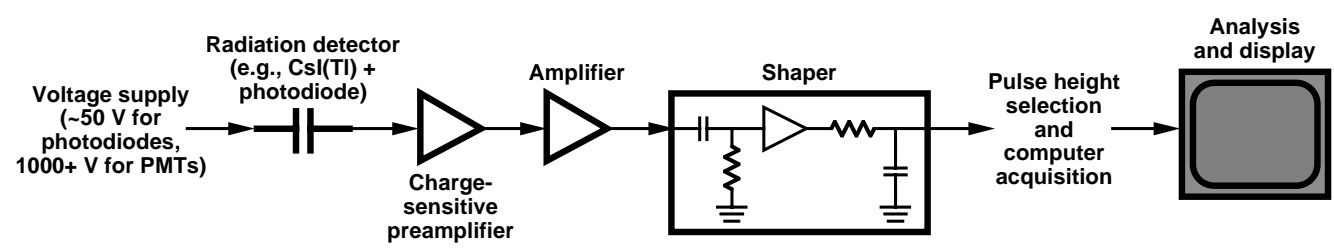

Figure 4.1: Basic components of a counting system used in nuclear medicine imaging. Oftentimes the amplifier and shaper stages can be combined. The CR differentiation stage in the shaper determines the fall time of the output signal, while the RC integration stage determines the rise time.

\subsubsection{Basic Pulse Counting Systems}

Radiation detectors used in nuclear medicine to count individual events (i.e., virtually all single photon and PET cameras) are operated in "pulse mode," wherein they produce electrical pulses which are subsequently counted and analyzed. The amplitudes of these pulses are used to measure gamma ray energy and thereby allow energy discrimination, so it is imperative that the readout system produces pulse amplitudes that suffer minimal fluctuations due to electronic noise. Figure 4.1 displays the basic components in most counting systems.

The charge-sensitive preamplifier, or preamp, is used to convert the very small current signals produced by the radiation detector $\left(<10,000 \mathrm{e}^{-}\right.$for most scintillator systems) into a voltage waveform, typically amplifying the signal in the process. The preamp must match both its input impedance with the typically large detector impedance and its output impedance with the electronics that follow immediately after the preamp. The preamp also performs a small amount of pulse shaping as it converts the input charge to an output voltage, and this must be compatible with all subsequent stages. An ideal charge-sensitive preamp is depicted in Figure 4.2, including the amplifier component, the detector capacitor $C_{d}$, and the feedback capacitor $C_{f}$. When a radiation event interacts in the detector, a charge $Q_{t o t a l}$ results from the formation of electron-hole pairs and is swept toward ground by the bias 


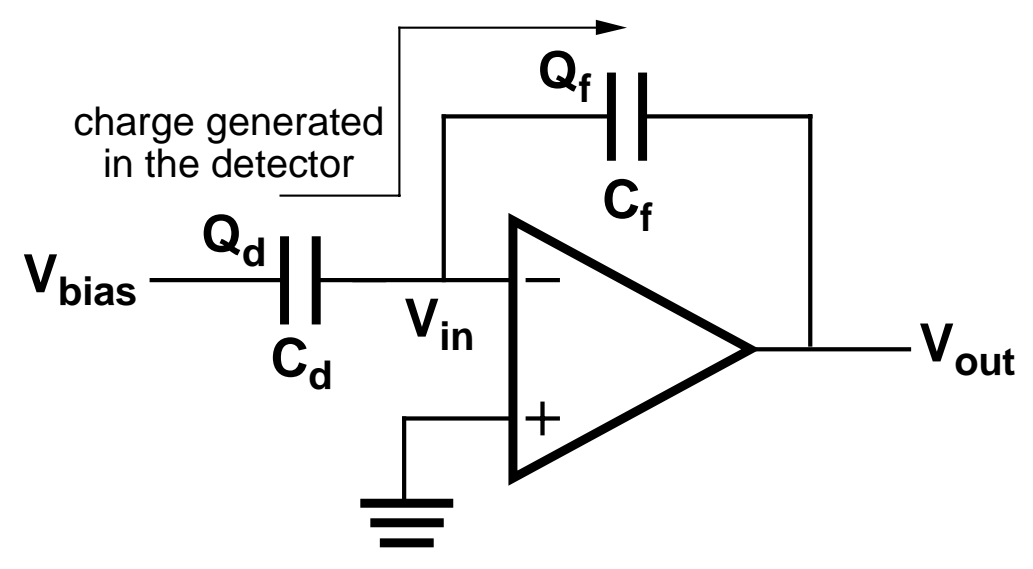

Figure 4.2: An ideal charge-sensitive preamp. Its amplifier component has infinite gain, infinite input impedance, zero output impedance, and does not require a feedback resistor to avoid saturation.

voltage. Assuming infinite amplifier input impedance, this charge distributes itself across the two capacitors according to the following equation:

$$
\frac{Q_{d}}{Q_{f}}=\frac{C_{d}}{(1+g) C_{f}}
$$

where $Q_{d}$ is the charge on $C_{d}, Q_{f}$ is the charge on $C_{f}$, and $g$ is the gain of the amplifier element. In the ideal case the gain, $g$, is infinite and forces all of the input charge from the photodetector, $Q_{\text {total }}$, onto the feedback capacitor. This then produces the following relationship for the output voltage:

$$
V_{\text {out }}=\frac{Q_{\text {total }}}{C_{f}}
$$

The output voltage is thus linearly related to the input charge, potentially with a large gain factor if $C_{f}$ is made small enough. Reality quite obviously differs substantially from this ideal analysis, however, and non-ideal complications are explored in Section 4.1.2.

The shaper stage is designed primarily to reshape the output of the preamplifier so that a good balance is achieved between: 
- dead time

- maintaining linear dependence of pulse amplitude on gamma ray energy

- maximizing signal

- minimizing noise (i.e., noise filtering)

- timing accuracy of pulse arrival.

The output of the preamplifier is typically a pulse with a $\sim 1 \mu$ s rise time and an exponential decay that can take 100 s of $\mu$ s to return to baseline. The shaper, however, typically shortens the pulse before it is sent to the PHA, usually to a total duration of only about 5-50 $\mu \mathrm{s}$, depending on the hardware and the application.

The second concern, linearity, can be met by employing a relatively simple system composed of a CR differentiation stage followed by an RC integration stage, as shown in Figure 4.3. The output pulse height for such a configuration is linearly dependent on the height of the input step signal. Additionally, by setting the differentiation and integration time constants appropriately, it is possible to alter the pulse duration (dead time), the pulse amplitude, and the noise. Pulse amplitude in the ideal case is independent of the time constants if the rise time to fall time ratio is kept constant, but in reality the amplitude is subject to losses due to ballistic deficit. This occurs when the rise time of the waveform coming out of the preamp is of comparable magnitude as the pulse duration, which is very possible when dealing with slow detectors like CsI(Tl) with its 0.8 and $3 \mu$ s scintillation light decay components. In this scenario the effective preamp rise time is slowed and only part of the preamp signal contributes to the shaper output pulse, resulting in a lower pulse amplitude. Applications that require excellent energy resolution and/or make use of slow detectors (e.g., $\mathrm{CsI}(\mathrm{Tl}))$ therefore use long rise and fall times. Noise is influenced by the shaper because the CR stage acts as a high-pass filter and the RC stage serves as a low-pass filter, therefore changing the time constants manipulates the passband. 


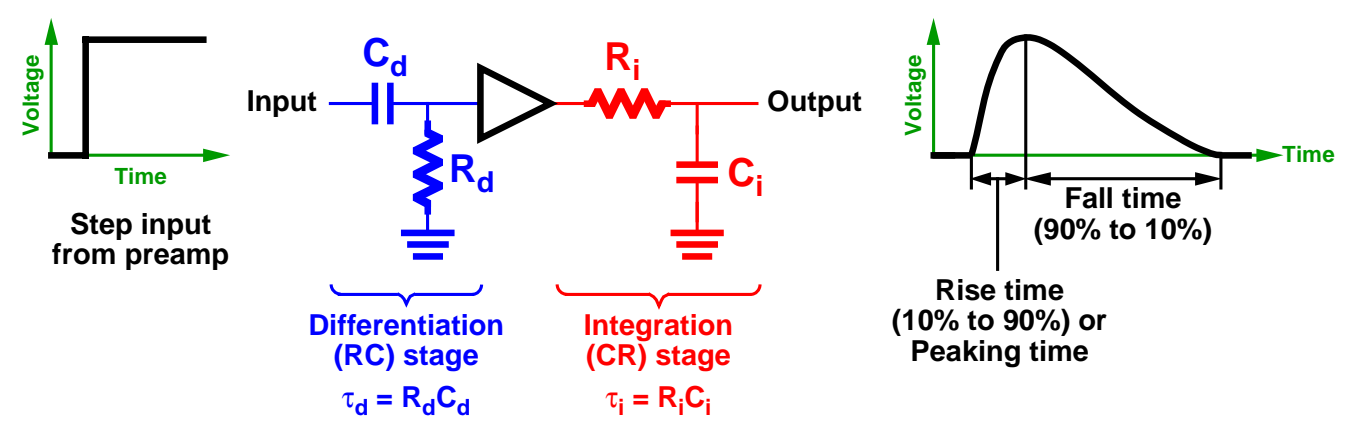

Figure 4.3: Shaper using a CR differentiation stage followed by an RC integration stage. The buffer may include some amplification, if desired. The two time constants can be manipulated to adjust the signal shape (i.e., rise time and fall time) and the noise filtering. Generally it is preferable to set $\tau_{d} \approx \tau_{i}$, in which case the peaking time is equal to about $1.2 \tau$ and the total pulse duration is about $7 \tau$. In reality the input step voltage from the preamp has an exponential decay to it, but this time constant is typically much greater than both $\tau_{d}$ and $\tau_{i}$ (the shaper output is supposed to be much shorter in duration than the preamp pulse) and can be safely ignored. Figure adapted from [13].

The final shaper concern, timing accuracy, is critical to PET instrumentation but is of little concern for single photon imaging systems. Applications like PET that require excellent timing resolution use short rise and fall times to achieve the best results (and therefore require fast scintillators).

\subsubsection{Electronic Noise}

Electronic noise must be carefully managed and minimized in order to ensure optimal performance in a pulse counting system, particularly where pulse height and energy resolution are concerned. Because PMTs have such large internal gain, their output signals are large enough to be immune to electronic noise in most circumstances. With photodiodes, however, the small signals are susceptible to electronic noise before being amplified by the preamp because any superimposed noise will be amplified along with the signal. After the preamp amplification, however, the signals 
gain a measure of security against the addition of further noise.

Reference [118] describes two distinct sources of noise in low-noise nuclear pulse amplifiers with a high input impedance (to match high impedance gamma ray detectors): step noise and delta noise. Both result from the discrete nature of electrical current - since current is the flow of individual electrons it is therefore subject to statistical behavior and fluctuations (shot noise). A third noise source, flicker or $1 / \mathrm{f}$ noise, is the result of material properties and is difficult to minimize beyond careful selection of substrate materials (e.g., ceramic printed circuit boards demonstrate less flicker noise than do FR4 boards).

Step noise is due to the current flowing in the input of the preamplifier, most commonly leakage current in the detector element and any current used to sink that leakage current in order to prevent preamplifier saturation. The name step noise derives from the fact that the fluctuations due to the discrete nature of the current are integrated by the input capacitance (capacitance of the detector element + input capacitance of the preamp) and appear as very small voltage steps at the preamp input. Because of the integration effect, the net injected charge is non-zero. Obviously these small, random voltage steps are amplified and appear at the output as noise.

Delta noise is due either to electron thermal agitation in a resistance near the preamp input or to current flowing within the preamp circuitry itself (most notably the bias current of the input transistor). These fluctuations at the preamp input are amplified and appear at the shaper output as unwanted noise, but unlike with step noise the duration of each individual fluctuation is relatively short and the signal quickly returns to baseline - there is no capacitive integration and the net injected charge is zero. A random series of brief delta-like noise pulses are observed in the output signal, hence the name delta noise. Unit noise sources for both step and delta noise are depicted in Figure 4.4.

A rudimentary analysis of the impact of step noise follows. Each step noise fluctuation occurring at time $t$ generates a shaper output signal $R_{\text {step }}(t)$ with a 


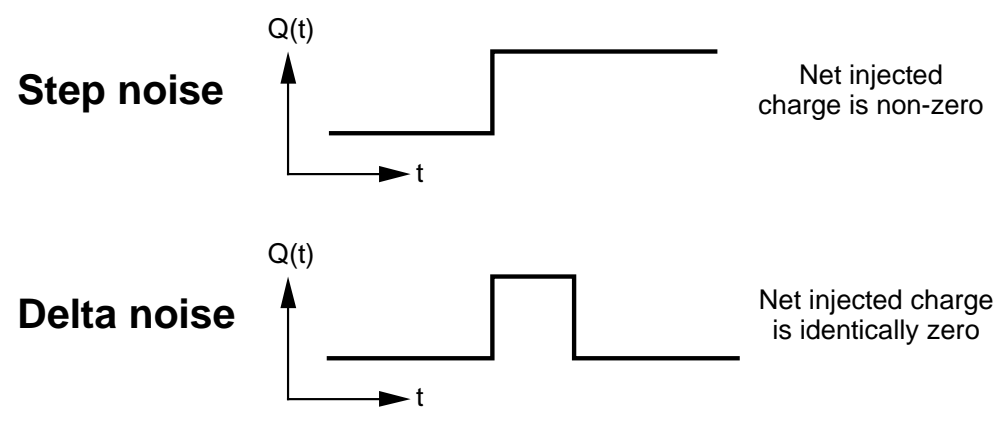

Figure 4.4: Unit step and delta noise sources.

duration of $\tau$ determined by the rise and fall times of the shaper. In incremental time $d t$ there are $N_{\text {step }}$ noise steps, which produce an incremental noise of $\sqrt{N_{\text {step }}} R_{\text {step }}(t)$. Adding all of these fluctuations in quadrature provides an equation for the noise:

$$
\left(\text { noise }_{\text {step }}\right)^{2}=\int_{0}^{\infty}\left(\sqrt{N_{\text {step }}} R_{\text {step }}(t)\right)^{2} d t .
$$

Making the reasonable assumption that the area beneath the curve $R_{\text {step }}(t)^{2}$ is proportional to $\tau$ (this should be true if the ratio of rise time to fall time is constant for all values of $\tau$ ), Equation 4.3 reduces to:

$$
\text { noise }_{\text {step }} \propto \sqrt{N_{\text {step }} \tau} \text {. }
$$

A similar analysis for delta noise assumes that each noise fluctuation is actually a step noise fluctuation followed by another step fluctuation of the opposite polarity at incremental time $d t$ later (resulting in zero net injected charge). The output of the shaper is therefore given by:

$$
R_{\text {delta }}(t)=R_{\text {step }}(t)-R_{\text {step }}(t+d t) .
$$

As with step noise, in incremental time $d t$ there are $N_{\text {delta }}$ delta noise pulses producing a noise of $\sqrt{N_{\text {delta }}} R_{\text {delta }}(t)$. Adding the fluctuations in quadrature yields:

$$
\left(\text { noise }_{\text {delta }}\right)^{2}=\int_{0}^{\infty}\left(\sqrt{N_{\text {delta }}} R_{\text {delta }}(t)\right)^{2} d t .
$$




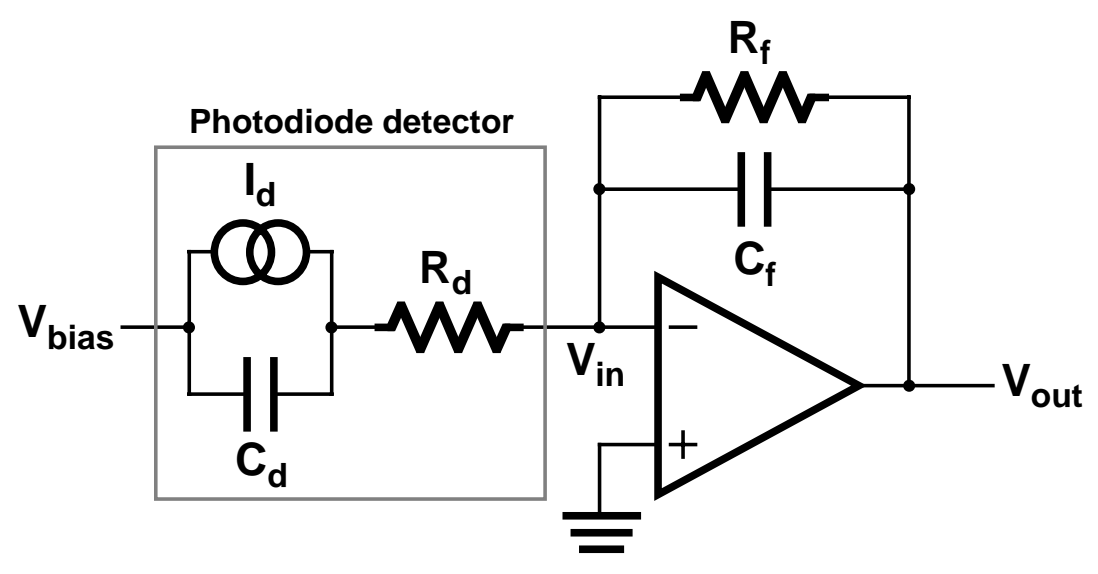

Figure 4.5: A charge-sensitive preamp and photodiode detector including noise sources. The detector leakage current, $I_{d}$, detector capacitance, $C_{d}$, detector series resistance, $R_{s}$, and preamplifier feedback resistor, $R_{f}$, all contribute unwanted noise. The first three are unavoidable material properties of photodiodes - they should be minimized but cannot be eliminated. The feedback resistor, $R_{f}$, is required in order to sink the detector leakage current and prevent amplifier saturation.

Making the reasonable assumption that the area beneath the curve $R_{\text {delta }}(t)^{2}$ is proportional to $1 / \tau$ (again, this should be true if the ratio of rise time to fall time is constant for all values of $\tau$ ), Equation 4.6 reduces to:

$$
\text { noise }_{\text {delta }} \propto \sqrt{\frac{N_{\text {delta }}}{\tau}}
$$

Figure 4.2 presented an ideal preamp, and now Figure 4.5 expands upon that by including non-ideal noise sources, namely detector leakage current, detector capacitance, detector series resistance, and the preamp feedback resistor used to sink detector leakage current and prevent amplifier saturation. The flow of leakage current in the detector, $I_{d}$, contributes step noise with $N_{\text {step }} \propto I_{d}$, hence:

$$
\text { noise }_{\text {leakage }} \propto \sqrt{I_{d} \tau} \text {. }
$$

The thermal noise voltage across a resistor is equal to $\sqrt{4 k T R \Delta f}$, where $k$ is Boltzmann's constant, $T$ is the temperature, and $\Delta f$ is the bandwidth. Thermal 
agitation in the detector series resistance, $R_{d}$, therefore generates delta noise with an input charge equal to the thermal voltage noise times the detector capacitance, $C_{d}$ :

$$
\text { noise }_{\text {series }} \propto C_{d} \sqrt{\frac{k T R_{d}}{\tau}} .
$$

The feedback resistor, $R_{f}$, used to sink the detector current is a source of additional current whose shot behavior is integrated by the input capacitance, resulting in extra step noise. The resistor generates thermal current equal to the thermal agitation voltage divided by the resistance, $R_{f}$. The resulting step noise is:

$$
\text { noise }_{\text {feedback }} \propto \sqrt{\frac{k T \tau}{R_{f}}} .
$$

Another means of sinking the detector leakage current would be to eliminate the feedback resistor and employ an opposite polarity current source adjusted to magnitude $I_{d}$ at the preamp input node. This eliminates the noise from Equation 4.10 but generates new step noise because of the shot noise in the sinking current. Because this current has magnitude $I_{d}$, the noise introduced is identical to that in Equation 4.8:

$$
\text { noise }_{\text {sink }} \propto \sqrt{I_{d} \tau}
$$

Thus both means of sinking the detector leakage current produce noise with magnitude proportion to $\sqrt{\tau}$.

The noise sources in Equations 4.8, 4.9, and 4.10 plus flicker noise (which is independent of the shaper pulse length, $\tau$ ) add in quadrature to produce the overall electronic noise:

$$
\text { noise }_{\text {total }}=\sqrt{A_{\text {series }} \frac{k T R_{d} C_{d}^{2}}{\tau}+A_{\text {flicker }}+A_{\text {parallel }}\left(I_{d}+\frac{k T}{R_{f}}\right) \tau}
$$

or, if the feedback resistor is eliminated in favor of a sinking current source as in Equation 4.11:

$$
\text { noise }_{\text {total }}=\sqrt{A_{\text {series }} \frac{k T R_{d} C_{d}^{2}}{\tau}+A_{\text {flicker }}+A_{\text {parallel }}\left(2 I_{d} \tau\right)}
$$


where $A_{\text {series }}, A_{\text {flicker }}$, and $A_{\text {parallel }}$ are constants. In either of these two cases there are three distinct terms within the square root. The first, commonly called series noise, is proportional to $R_{d} C_{d}^{2}$ and decreases as $\tau$ increases. The second, flicker noise, is independent of $\tau$ and sets a lower bound for the noise. The third is often referred to as parallel noise and increases as $\tau$ increases. It can be minimized by reducing $I_{d}$, which is the primary motivation for using low-leakage current photodiodes in a $\operatorname{CsI}(\mathrm{Tl})$ detector system. Finally, note that while Equations 4.12 and 4.13 were derived with $\tau$ equal to the total pulse duration of the shaper output, the shaper peaking time is proportional to $\tau$ and therefore these equations are equally valid with peaking time substituted for $\tau$ as long as the constant values $A_{\text {series }}, A_{\text {flicker }}$, and $A_{\text {parallel }}$ are changed appropriately. Figure 4.6 plots the electronic noise of Equations 4.12 and 4.13 versus shaper peaking time using constant values consistent with the behavior of the $\mathrm{CsI}(\mathrm{Tl}) / \mathrm{Si}$ PIN photodiode/prototype ICs detector system described in this chapter.

\subsection{Prototype Detector System}

\subsubsection{Module Design}

The $3 \times 4$ pixel prototype imaging module I constructed and characterized is depicted in Figure 4.7. A hexagonal hole lead collimator provides directional information, discrete $\mathrm{CsI}(\mathrm{Tl})$ crystals convert incident gamma rays to scintillation light, a photodiode array detects these scintillation photons, and two ASICs read out the photodiode signals in preparation for computer acquisition. The photodiode arrays were designed for low leakage current, which is critical to minimizing electronic noise (as per Equations 4.12 and 4.13$)$ at the long peaking times $(\sim 8 \mu \mathrm{s})$ that are necessary when using $\mathrm{CsI}(\mathrm{Tl})$ crystals.

A prototype pixel size of $3 \times 3 \mathrm{~mm}^{2}$ was chosen as a compromise between several factors. A smaller pixel size both provides slightly better intrinsic spatial 


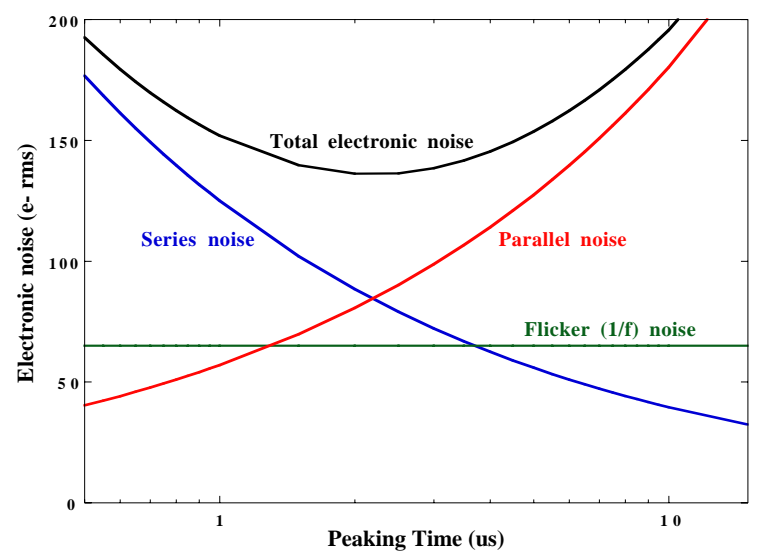

Figure 4.6: Electronic noise versus shaper peaking time. The dependence of the total electronic noise and the three individual noise components on the peaking time is obvious. The noise minimum for this particular graph occurs at about $2 \mu \mathrm{s}$. While this would seem to be an attractive operating point, the long scintillation decay times of $\mathrm{CsI}(\mathrm{Tl})$ generally mean that better signal-to-noise can be achieved at greater peaking times because short shaping times cause ballistic deficit signal losses. Further, the noise penalty incurred at slightly longer shaping times is typically mild.

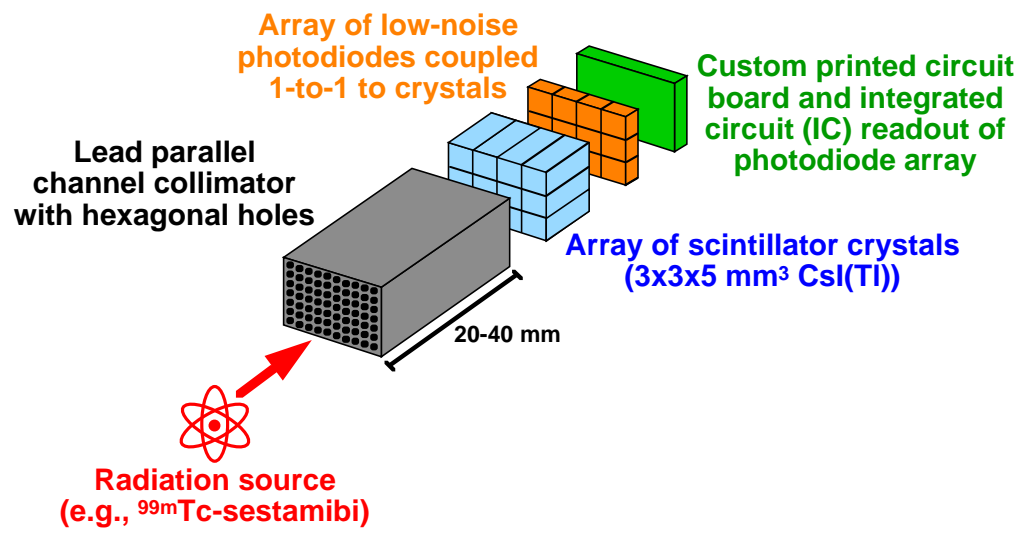

Figure 4.7: Prototype discrete scintillation camera module with $3 \times 4$ pixels. Each pixel element is comprised of a $3 \times 3 \times 5 \mathrm{~mm}^{3} \mathrm{CsI}(\mathrm{Tl})$ crystal coupled via transparent epoxy to a $3 \times 3 \mathrm{~mm}^{2}$ Si PIN photodiode. The readout circuitry consists of the two $3 \times 3 \mathrm{~mm}^{2}$ ASICs described in Section 4.2.3. 
resolution (though this benefit is limited by the fact that the collimator, not pixel size, tends to be the limiting component) and yields less leakage current and capacitance per pixel, which lowers electronic noise (again, see Equations 4.12 and 4.13). However, using smaller pixels also increases the pixel density and hence the density of electronics required to read out the entire array, which becomes a very significant problem in larger arrays of useful imaging size. Studies on the optimal pixel size from a breast imaging perspective are presented in Chapter 5.

\subsubsection{Prototype Low-noise Photodiodes}

The custom low-noise photodiodes were made on $300 \mu \mathrm{m}$ thick, high resistivity (>10 k $\Omega$-cm), n-type silicon. When fully depleted they have a quantum efficiency of about $90 \%$ for the $540 \mathrm{~nm}$ scintillation light produced by $\mathrm{CsI}(\mathrm{Tl})$ and demonstrate room temperature leakage currents of $0.2-0.6 \mathrm{nA} / \mathrm{cm}^{2}$. These leakage currents are significantly lower than anything currently available from commercial photodiodes and are achieved by depositing a thick layer of phosphorus-doped polysilicon on the silicon wafer in the early processing stages to getter impurity atoms, removing them from the substrate silicon and thereby decreasing the production of electron-hole pairs [121]. The $\mathrm{p}+$ junctions are fabricated by ion-implanting boron, while the $\mathrm{n}+$ back contact consists of a second, thin layer of polysilicon deposited after the gettering polysilicon layer is etched away. Finally, a layer of indium tin oxide (ITO) is deposited on the polysilicon backside to reduce the back contact resistance and to serve as an antireflective (AR) coating. The AR coating is optimized for $540 \mathrm{~nm}$ light - the peak output of $\mathrm{CsI}(\mathrm{Tl})$ — and is therefore $679 \AA$ thick. This is $1 / 4$ the wavelength of $540 \mathrm{~nm}$ light after it has entered ITO, which has an index of refraction of nearly 2 , and therefore produces constructive interference of the reflected light. I have verified that after dicing the individual photodiode arrays to remove them from the fabrication wafers and then mounting the arrays on custom printed circuit boards, $3 \times 3 \mathrm{~mm}^{2}$ pixels demonstrate a capacitance of about $3 \mathrm{pF}$ and leak- 
age current of about $50 \mathrm{pA}$ at room temperature and $50 \mathrm{~V}$ bias. The arrays used in the prototype detector module are $3 \times 4$ pixels in size, but for the final imaging modules these arrays have been successfully scaled up to 64 pixels and demonstrate similar electrical characteristics.

\subsubsection{Prototype Readout ICs}

The two ASICs used with the 12-pixel detector element are prototype ICs for the final 64-pixel readout chip. A great deal of my work with the prototype module involved debugging, characterizing, and optimizing these ICs and their communication with each other. Both are CMOS designs fabricated in the HP $1.2 \mu \mathrm{m}$ process and are about $3 \times 3 \mathrm{~mm}^{2}$ in area. The final 64 -channel version incorporates the major elements from both prototype ICs plus various improvements, is fabricated in the HP $0.5 \mu \mathrm{m}$ process, and is about $4.5 \times 5 \mathrm{~mm}^{2}$ in size. Brief descriptions of the two prototype ICs are given below, but for more detailed information and circuit diagrams regarding this IC readout scheme, refer to the discussion of the final 64-channel IC in Chapter 6.

The first prototype IC is a 16-channel preamplifier and shaper amplifier front end. External current controls allow adjustment of a preamp compensation current (to sink the detector leakage current and prevent preamp saturation), the shaper rise time, and the shaper fall time. Lower noise could potentially be achieved using a feedback resistor rather than an additional current source to sink the detector current, but only if the value of $R_{f}$ were made very large (see Equation 4.12 ), typically on the order of $1 \mathrm{G} \Omega$. Unfortunately, resistors this large are extremely difficult to fabricate in a CMOS integrated circuit, so the compensation current source proves the better choice. The preamps in the final 64-pixel IC actually do eliminate the extra current source by using a transistor as a voltage-controlled resistance in place of a true resistor, $R_{f}$. This provides for a potentially large and therefore low-noise resistance, but it also requires careful and somewhat complicated control of the 
transistor. Even with this aggressive scheme, however, the noise gains are small.

My observations with the front end IC have shown that a rise time to fall time ratio of about 1:3 provides the greatest signal-to-noise, though ratios ranging from as low as 1:2 to as high as 1:5 yield fairly similar results. These measurements were made by injecting a known charge into the front end IC with a step voltage pulse and a capacitor, collecting a histogram of the output pulse peak values, determining the signal level (in $\mathrm{e}^{-}$) as the center of the histogram peak, calculating the electronic noise (in $\mathrm{e}^{-}$) from the histogram peak fwhm, and then simply computing the signalto-noise ratio. The rise time to fall time ratio of $1: 3$ is slightly different than the $\sim 1: 5$ rise time to fall time ratio that results if the differentiation time constant and integration time constant are made equal, as suggested in Figure 4.3. The preamps in this IC can successfully achieve the 1:3 rise to fall ratio for a range of peaking times from $0.5 \mu \mathrm{s}$ to $15 \mu \mathrm{s}$. Further, the preamps can handle input loads in excess of $5 \mathrm{pF}$ and $300 \mathrm{pA}$, which is more than adequate to handle the custom low-noise photodiodes and their loads of about $3 \mathrm{pF}$ and $50 \mathrm{pA}$. Care must be taken with large input currents, however, because the preamp reset time begins to shorten. This can become problematic if it drops much below $50 \mu \mathrm{s}$ because then the input to the shaper can no longer be assumed to be a step voltage and the shape of the output waveform becomes distorted. Finally, this front end IC offers a range of overall gains which can be set externally with jumpers. The maximum gain is approximately $100 \mathrm{mV} / 1000 \mathrm{e}^{-}$.

The 16-channel "Winner Take All," or WTA, IC is essentially a multiplexer that selects the input with the greatest amplitude. It accomplishes this by having each input voltage control the gate voltage of a field effect transistor (FET). All 16 of these transistors "fight" each other for a common, limited current supply of about $30 \mu \mathrm{A}$, and because of the square-law dependency of drain current on gate voltage in a FET, the transistor with the greatest gate voltage will take the majority of this current. This then identifies the winning channel, whose voltage waveform the IC places on an analog output line. A 6-bit digital address indicates which channel 
is the present winner. The WTA IC correctly distinguishes between signals with amplitude differences as small as $20 \mathrm{mV}$ and, when the winning signal changes, it correctly switches the output address within 50 ns.

\subsubsection{Readout and Computer Acquisition}

The basics of the computer acquisition scheme I implemented are shown in Figure 4.8 (minus most of the level shifting and peak detect details). The photodiode signals are amplified and shaped by the 16-channel prototype front end IC, then processed by the 16-channel prototype WTA IC. The WTA selects the signal with the greatest amplitude and places it on the analog output line, thereby identifying the crystal of interaction (pixel address) and the incident photon energy (signal amplitude). The analog "winner" signal selected by the WTA is sent to a threshold discriminator, and the pulse height is read out with a peak detecting CAMAC ADC. At the same time, the 6-bit digital address computed by the WTA to identify the "winner" channel is read out with a CAMAC I/O board, and both the digitized signal amplitude and the corresponding address are collected by an acquisition computer.

\subsubsection{Collimator Design}

Since the collimator is the limiting factor for both spatial resolution and sensitivity in single photon imaging devices, careful collimator design is crucial. Traditional scintillation cameras use hexagonal hole collimators, but in a discrete scintillator camera it is also possible to use square holes matched 1-to-1 (or 4-to-1, etc.) to the square detector pixels. Early simulations suggested that for square pixel detector arrays, matched square holes provide a superior spatial resolution/sensitivity tradeoff compared to hexagonal holes [96]. Additionally, matching square collimator holes to the detector pixels provides a point spread function with minimal dependence on source position by eliminating the aliasing due to geometric mismatch between hexagonal collimator holes and square detector pixels. Hexagonal hole collimators, 


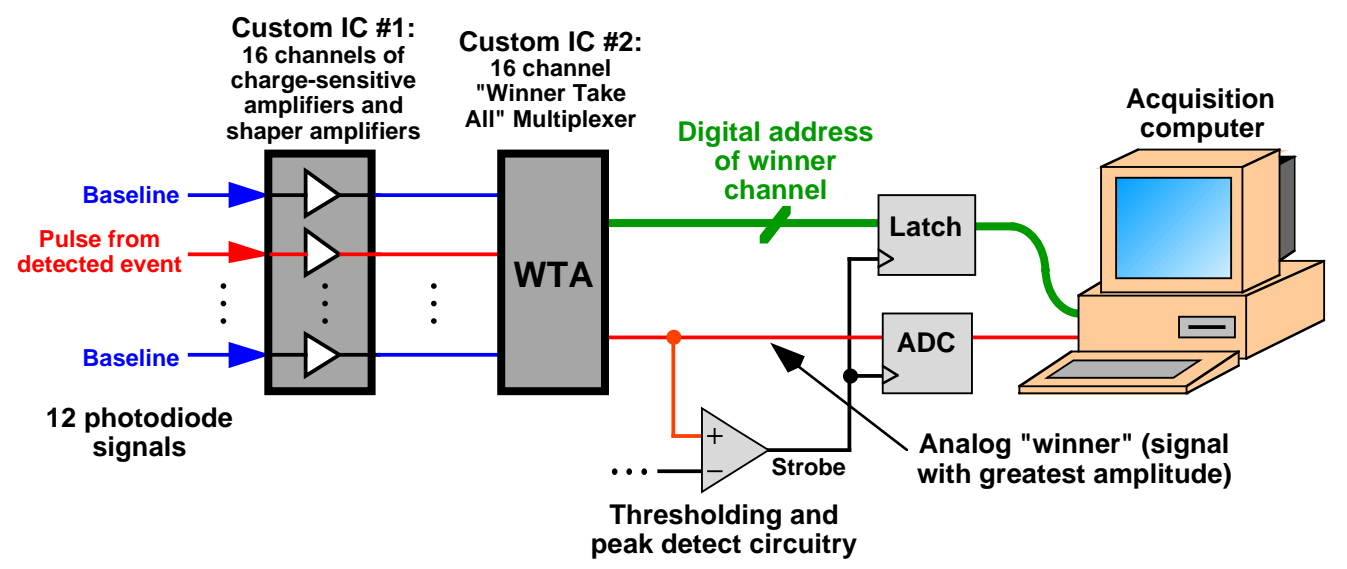

Figure 4.8: ASIC readout of the photodiode array and computer acquisition for the prototype detector module. The first IC amplifies and shapes the photodiode signals, while the WTA IC selects the signal with the greatest amplitude and passes it plus a 6-bit digital address denoting the "winner" channel on to an acquisition computer. The computer performs energy discrimination and integrates the data points into a grayscale image. 
however, have a more symmetric septal penetration pattern and can generally result in shorter collimators for the same sensitivity because hexagonal holes can usually be made smaller than matched square holes - the former do not share the latter's constraint of matching to the size (or $1 / 4$ the size) of the detector pixels).

Recall from Equation 1.4 that to first order the spatial resolution of either a hexagonal or square hole collimator is:

$$
R_{\text {collimator }} \approx d\left(\frac{l+b}{l}\right)=d+\frac{d b}{l}
$$

where $d$ is the hole diameter, $l$ is the hole length, and $b$ is the collimator-to-object imaging distance. This equation thus loosely defines two imaging ranges: the near field $(b<l)$, where the first term is dominant and resolution is equal to the collimator hole diameter, $d$, and the far field $(b>l)$, where the second term is dominant and the resolution is equal to $d b / l$. An imaging distance on the order of $50-100 \%$ of the collimator height - or in range of 5-30 mm for many of the compact collimators that might be considered for compact gamma cameras - is the approximate crossover point between the two.

In the far field the resolution is limited by the collimator aspect ratio, assuming the intrinsic resolution is sufficiently fine. In the near field gamma rays from a point source centered over a collimator hole tend to penetrate exactly one hole, so if the collimator is matched to the detector pixel, the spatial resolution is determined by the pixel size and not the collimator aspect ratio. Since the collimator sensitivity is approximately proportional to the square of the collimator aspect ratio for both the near and far field, the classical collimator resolution/sensitivity trade-off (Equation 1.5) does not hold in the near field and finer resolution can be achieved without compromising sensitivity by reducing $d$ and $l$ proportionally. Given discrete, square detector pixel elements, this advantage can be fully realized with a matched square hole collimator, but only partially realized with a hexagonal hole collimator because geometric mismatch can allow two or more detector pixels to be exposed to gamma rays even if only one collimator hole is penetrated. 
Potential for improving the resolution/sensitivity trade-off thus exists when the object is in the near field of the collimator, but the depth of the near field is less than the length of the collimator, $l$. Finer collimator spatial resolution in this region can be achieved by reducing the hole diameter $d$, but maintaining high sensitivity requires a corresponding reduction in $l$, which then reduces the extent of the near field. As was mentioned above, with the relatively short collimator geometries employed for most compact gamma camera systems, the collimator near field cannot be assumed to extend further than $30 \mathrm{~mm}$, with depths of 5-15 mm being much more common. For scintimammography it is necessary to image breast lesions at distances as great as 5-6 $\mathrm{cm}$ (the depth of the breast under mild compression), so it is impossible to rely upon the near field benefits to provide superior performance across the entire imaging range. Hence both near and far field performance must be considered when designing a collimator geometry, and any device that claims to have drastically improved upon traditional collimator performance deserves a critical eye.

I performed the measurements presented in this thesis using hexagonal hole collimators primarily because of their present commercial availability. As was previously mentioned, tungsten laminate collimators are now being fabricated with a wide variety of hole shapes, including square, but for the time being are about an order of magnitude more expensive.

My Monte Carlo simulations (precursors to those in Chapter 5) indicate that if the hexagonal collimator holes are small relative to pixel size, namely:

$$
\text { hole diameter }<0.5 \times \text { pixel size, }
$$

then the spatial resolution degradation due to the aliasing error between hexagonal collimator holes and square detector pixels is small. This issue will be further addressed and quantified in Sections 4.3.4 and 4.3.5. Even so, there remains the complication that the septal pattern on top of the pixels (which decreases sensitivity) differs from pixel to pixel, and therefore calibration with a flood source should be used to account for pixel-to-pixel variations in sensitivity. 


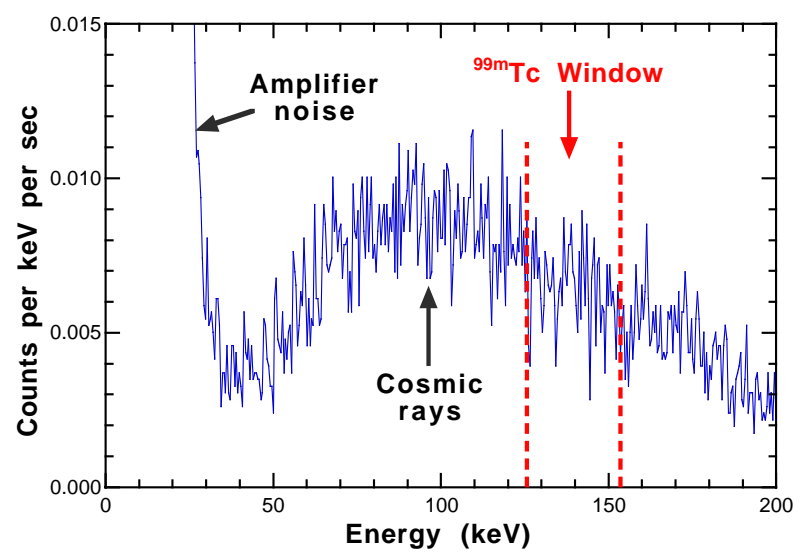

Figure 4.9: Background spectrum for all 12 pixels in the prototype detector array. There are 0.24 counts/channel/sec above $50 \mathrm{keV}$, and 0.015 counts/channel/sec in the $126-154 \mathrm{keV}^{99 m} \mathrm{Tc}$ window.

\subsection{Detector Imaging Characteristics}

\subsubsection{Background Spectrum}

The $3 \times 4$ prototype array demonstrates low count rates when there is no radiation source present. The detector (without a collimator) was carefully wrapped in grounded aluminum foil to shield it from electromagnetic fields (EMF), a $50 \mathrm{~V}$ bias was connected to the photodiode array, and the entire system was placed in darkness under a thick dark cloth. The average count rate across all 12 pixels is 0.24 events/pixel/sec above a $50 \mathrm{keV}$ threshold, and 0.015 counts/pixels/sec within the $126-154 \mathrm{keV}$ energy window commonly used for ${ }^{99 m} \mathrm{Tc}$. This rate of background activity is consistent with cosmic ray flux. The summed spectrum for the entire array is shown in Figure 4.9. 


\subsubsection{Energy Resolution}

To allow observation of energy resolution, the prototype detector array (without a collimator) was again carefully wrapped in grounded aluminum foil to EMF shield it, $50 \mathrm{~V}$ bias was applied to the photodiodes, and the entire system was placed in darkness under a thick black cloth. A $2 \mathrm{~mm}{ }^{99 m} \mathrm{Tc}$ source was placed about $5 \mathrm{~cm}$ from the detector array to excite it, producing a count rate of about $10 \mathrm{~Hz}$ per pixel. The 12 prototype detector pixels demonstrate an average room temperature energy resolution of $11.7 \pm 0.9 \%$ fwhm for the $140 \mathrm{keV}$ gammas when using amplifier shaping times of $8 \mu$ s rise and $24 \mu$ s fall. However, the $530 \AA$ thickness of the anti-reflective coating on the photodiode array used in this module was optimized for $410 \mathrm{~nm}$ light (the peak intensity produced by lutetium oxyorthosilicate scintillator, also known as LSO, which is commonly used in PET detectors) and not for the $540 \mathrm{~nm}$ emissions of $\mathrm{CsI}(\mathrm{Tl})$, preventing complete collection of the scintillation photons. Calibration using the direct interaction of $5.9 \mathrm{keV}{ }^{55} \mathrm{Fe}$ gammas in silicon photodiodes (and assuming $3.6 \mathrm{eV}$ per electron-hole pair generation) showed that the average signal amplitude in these measurements was $5400 \mathrm{e}^{-}$. This value is very similar to the results reported in [122], wherein various cylindrical $\mathrm{CsI}(\mathrm{Tl})$ crystals (9 mm diameter, 1-9 mm height) were read out with Hamamatsu S3590-3 and S2744-04 photodiodes (Hamamatsu Photonics K.K., Hamamatsu City, Japan) demonstrate an average signal level of $5387 \mathrm{e}^{-}$.

A second $3 \times 4$ detector array using an identical $\mathrm{CsI}(\mathrm{Tl})$ array coupled to a photodiode array with an AR coating optimized for $\mathrm{CsI}(\mathrm{Tl})$ (679 $\AA$ thickness) was also tested. The average signal amplitude increased to $6600 \mathrm{e}^{-}$, and the average room temperature energy resolution at $140 \mathrm{keV}$ dropped to $10.7 \pm 0.6 \%$ fwhm at $140 \mathrm{keV}$. Various photopeaks for this array are shown in Figure 4.10. The measurements of spatial resolution reported in the remainder of this chapter, however, were made on the first detector array with the slightly lower signal levels.

I determined that the choice of $\sim 8 \mu$ s peaking time ( $8 \mu$ s rise, $24 \mu$ s fall $)$ 


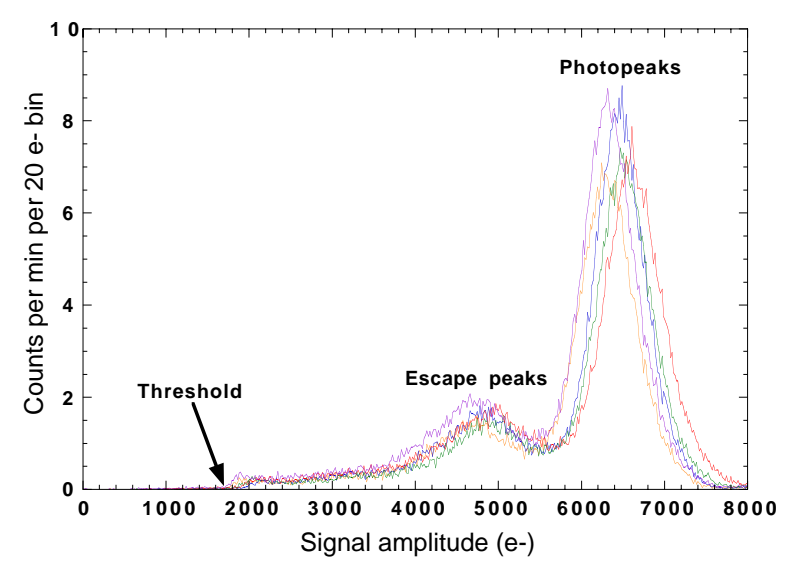

Figure 4.10: Room temperature ${ }^{99 m}$ Tc photopeaks for pixels in the prototype module. Amplifier shaping times were $8 \mu$ s rise and $24 \mu$ fall. The average signal amplitude is $6600 \mathrm{e}^{-}$and the average photopeak width $700 \mathrm{e}^{-}$, yielding average energy resolution for all arrays pixels of $10.7 \pm 0.6 \%$ fwhm.

provides the most accurate energy resolution, despite the fact that the noise minimum for the amplifier/photodiode electronics occurs in the $2-4 \mu$ s range, by observing the energy resolution for $140 \mathrm{keV}$ photons as described above for a range of peaking times from 1 to $12 \mu$. The minimum electronic noise in the peaking time range of 2-4 $\mu$ s is approximately $345 \mathrm{e}^{-}$fwhm, compared to $390 \mathrm{e}^{-}$fwhm at $8 \mu$ s (these noise values were measured by injecting a known charge into the detector array channels using a step voltage pulse and a capacitor connected to the IC inputs, histogramming the peak values of the resulting output waveforms, and calculating the fwhm in $\mathrm{e}^{-}$ of the resulting histogram peak). However, the slow decay components of $\mathrm{CsI}(\mathrm{Tl})$ scintillation light $(0.8$ and $3 \mu \mathrm{s})$ mean that more scintillation photons are collected at $8 \mu \mathrm{s}$ than are collected at $2-4 \mu \mathrm{s}$, and this increase in signal is larger than the associated increase in noise. At a peaking time of $8 \mu \mathrm{s}$, the electronic noise is $5.9 \%$ fwhm of the $6600 \mathrm{e}^{-}$signal. The statistical noise is only $2.8 \%$ fwhm, leaving an additional $8.5 \%$ fwhm contribution added in quadrature in order to account for the average photopeak width of $10.7 \%$ fwhm. Because the three major noise sources in 
scintillator/photodiode detectors are electronic, statistical, and inhomogeneities in light collection [123], this remaining $8.5 \%$ fwhm error is assumed to be due to light collection inhomogeneity in the $\mathrm{CsI}(\mathrm{Tl})$ crystals.

There is potential to further improve the energy resolution and match or even surpass the $9 \%$ fwhm achieved in traditional Anger scintillation cameras. The primary source of noise $(8.5 \% \mathrm{fwhm})$ appears to be due to inhomogeneity in light collection in the $\mathrm{Cs}(\mathrm{Tl})$, so reducing this would be the best starting point. With crystals only $5 \mathrm{~mm}$ in depth, it may be possible to reduce this source below the $5.9 \%$ fwhm contributed by the electronic noise (light collection inhomogeneity as low as 4.4\% fwhm has been reported with $\mathrm{CsI}(\mathrm{Tl})$ [124]). Higher quality $\mathrm{CsI}(\mathrm{Tl})$ arrays would be worth testing, as the crystals used in the prototype may suffer from depth of interaction effects (i.e., scintillation photons attenuate as they traverse the crystal) and suboptimal surface quality (preventing some scintillation photons from reaching the photodiode). If electronic noise at some point becomes the dominant source, it may be possible to reduce it by improving the charge amplifier IC design as well as its fabrication technology. Efforts along these lines are presented in Chapter 7 with the discussion of the final 64-pixel imaging modules. Finally, there remains the possibility of cooling the instrumentation to perhaps $5^{\circ} \mathrm{C}$ in order to lower leakage currents and reduce the parallel noise in the amplifier system. Similar CsI(Tl)/photodiode technology in a cooled environment has demonstrated an energy resolution of $7.5 \%$ fwhm for the $122 \mathrm{keV}$ emissions of ${ }^{57} \mathrm{Co}$ [94]. However, it is certainly preferable to avoid the need to cool the imaging device (which would add additional hardware and design challenges), if possible.

\subsubsection{Intrinsic Spatial Resolution}

I scanned a ${ }^{57} \mathrm{Co}$ beam across the central row of four crystals in the $\mathrm{CsI}(\mathrm{Tl})$ array (without a collimator) to observe intrinsic spatial resolution. The beam was formed by placing the ${ }^{57} \mathrm{Co}$ source behind a lead shield with a cylindrical hole that 
allowed some of the $122 \mathrm{keV}$ gammas to escape and excite the array. This cylindrical hole was about $1 \mathrm{~mm}$ in diameter and $10 \mathrm{~mm}$ in height, so when the source was scanned across the prototype detector at a distance of approximately $7.5 \mathrm{~mm}$, the cone of gamma rays emerging from the lead assembly had a diameter of about $2.5 \mathrm{~mm}$ on the face of the $\mathrm{CsI}(\mathrm{Tl})$ crystals. The responses of the four individual pixels are displayed in Figure 4.11 and demonstrate an average spatial resolution of $3.3 \mathrm{~mm}$ fwhm. To first order approximation, the spatial response is the pixel response (a square function) convolved with the source intensity (also a square function), resulting in a triangular function much like is evident in Figure 4.11. Thus, the intrinsic spatial fwhm should be the pixel pitch $(3 \mathrm{~mm})$ and the source diameter $(2.5 \mathrm{~mm}$ on the face of the detector) added in quadrature, yielding an estimate of $3.9 \mathrm{~mm}$ fwhm. The observed results are therefore slightly better than expected-possibly because the source/cylindrical hole geometry in actuality strayed from the values described above or because the source intensity is not well-modeled by a square function-and certainly imply that electronic and Compton crosstalk in the 12-pixel prototype are minimal.

\subsubsection{System Spatial Resolution with a High Resolution Collimator}

I evaluated the spatial resolution of the complete prototype module (including a high resolution hexagonal hole collimator) in air by scanning a $2 \mathrm{~mm}$ diameter uncollimated ${ }^{99 m}$ Tc source across the middle row of four pixels at imaging distances of $0.0,2.5$, and $5.0 \mathrm{~cm}$ from the front face of the collimator. This is the imaging range of interest for clinical applications because with mild breast compression, most tumors to be imaged will be within $5 \mathrm{~cm}$ of the collimator surface. The collimator has $1.5 \mathrm{~mm}$ diameter hexagonal holes and a length of $32 \mathrm{~mm}$, yielding a sensitivity of about 4300 events $/ \mathrm{mCi} / \mathrm{sec}$. The average spatial resolution of the four pixels is $4.1 \mathrm{~mm}$ fwhm at a distance of $0.0 \mathrm{~cm}, 4.8 \mathrm{~mm}$ fwhm at $2.5 \mathrm{~cm}$, and $5.9 \mathrm{~mm}$ fwhm at $5.0 \mathrm{~cm}$. Individual pixel responses are displayed in Figure 4.12. 


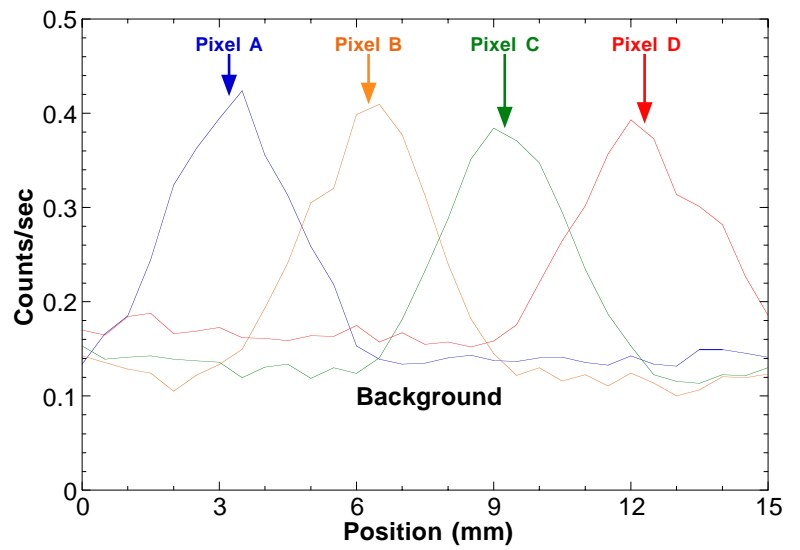

Figure 4.11: Response of the central row of four pixels to a $2.5 \mathrm{~mm}$ diameter ${ }^{57} \mathrm{Co}$ collimated beam scanned across the face of the $\mathrm{CsI}(\mathrm{Tl})$ array in the prototype module. The average spatial resolution is $3.3 \mathrm{~mm}$ fwhm. Significant background activity is apparent because the source activity was very weak at less than $20 \mathrm{nCi}$.

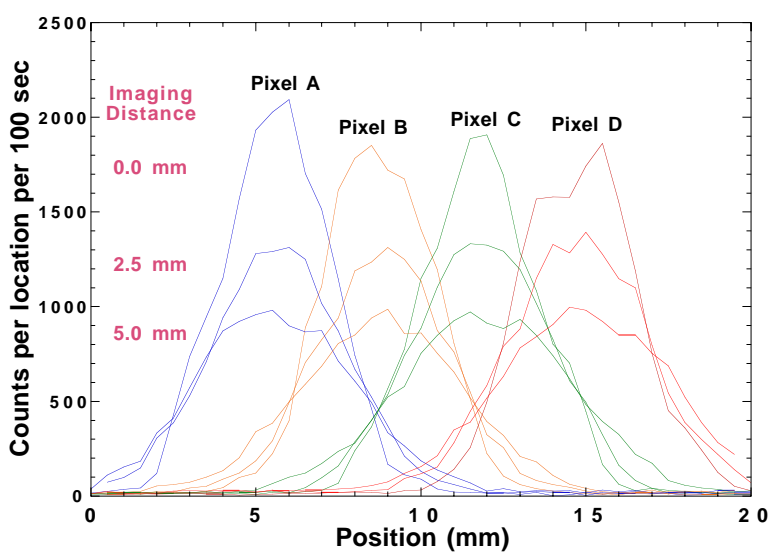

Figure 4.12: Response of the central row of four pixels behind a high resolution hexagonal hole collimator to a $2.0 \mathrm{~mm}$ diameter uncollimated ${ }^{99 \mathrm{~m}} \mathrm{Tc}$ source scanned across at imaging distances of $0.0,2.5$, and $5.0 \mathrm{~cm}$. The average spatial resolution at these distances is $4.1 \mathrm{~mm}, 4.8 \mathrm{~mm}$, and $5.9 \mathrm{~mm}$ fwhm, respectively. Measurements were performed in air. The collimator has a sensitivity of 4300 events $/ \mathrm{mCi} / \mathrm{sec}$. 


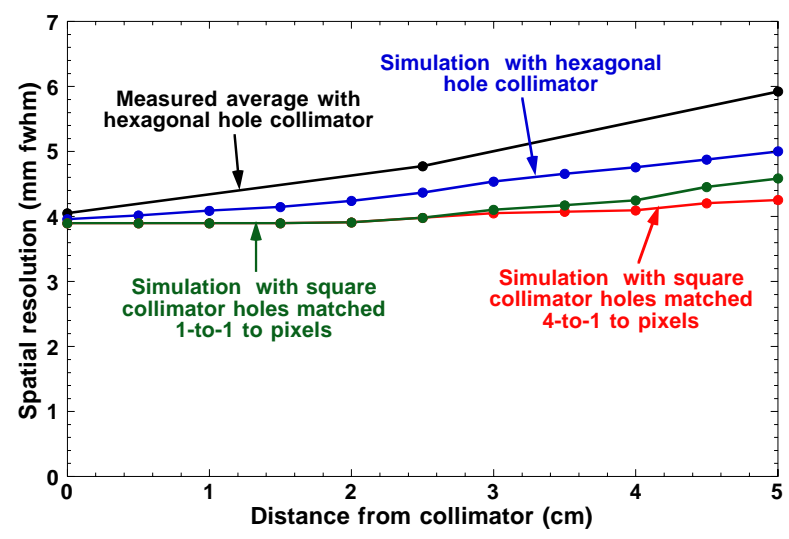

Figure 4.13: Average spatial resolution versus imaging distance for measured and simulated results with high resolution collimators. The simulated hexagonal hole collimator has an identical geometry to the experimental collimator, the 1-to-1 matched square hole collimator is $66 \mathrm{~mm}$ long with $2.8 \times 2.8 \mathrm{~mm}^{2}$ holes $(0.2 \mathrm{~mm}$ septal thickness makes unit cells $\left.3.0 \times 3.0 \mathrm{~mm}^{2}\right)$, and the 4 -to- 1 matched square hole collimator is $30 \mathrm{~mm}$ long with $1.3 \times 1.3 \mathrm{~mm}^{2}$ holes $(0.2 \mathrm{~mm}$ septal thickness makes unit cells $\left.1.5 \times 1.5 \mathrm{~mm}^{2}\right)$. All collimators have sensitivities of about 4300 events $/ \mathrm{mCi} / \mathrm{sec}$.

I performed Monte Carlo simulations of spatial resolution to compare measured results with theoretical predictions, as well as to compare a standard hexagonal hole collimator to a collimator with square holes matched either 1-to-1 or 4-to-1 to the square $\mathrm{CsI}(\mathrm{Tl})$ crystals. More advanced simulations built upon the framework of these Monte Carlo runs are described in Chapter 5. Figure 4.13 presents the spatial resolution averages measured with the 12-pixel array when equipped with a high resolution hexagonal hole collimator, as well as analogous simulated results for detectors equipped with both square and hexagonal hole collimators with approximately the same sensitivity.

The preliminary simulations used in this chapter compute the spatial resolution (with the results in the $\mathrm{x}$ and $\mathrm{y}$ directions weighted equally) averaged across 25 different point source locations in a $5 \times 5$ square grid with a pitch of $1 / 10$ the pixel width. This provides coverage of one quadrant of one pixel and was implemented 
in order to prevent advantageous or disadvantageous positions from distorting the results, as the width of the spatial response exhibits some dependence on the exact location of the point source (e.g., centered above a pixel versus above the border between two pixels). The magnitude of these variations are quantified near the end of this section. Coverage of one quadrant of one pixel was deemed sufficient for this task because of the symmetry that often exists with the other three quadrants.

The Monte Carlo simulations implement an infinitesimally small point source in air, fully realize finite septal thickness in the simulated collimator geometries, and assume that the septal lead in the collimator is $100 \%$ efficient at stopping gammas (because complicated septal penetration calculations were not yet implemented). Since positioning of the collimator holes relative to the square detector pixels affects the resulting spatial resolution, the code allows easy modification of this positioning. For square collimator holes the holes were, or course, matched either 1-to-1 or 4-to-1 to the detector pixels such that edges of the pixels were completely obscured by collimator septal walls. For hexagonal hole collimators, however, there are many possibilities for the relative positioning of the holes and the pixels. For the data presented in this chapter a hexagonal collimator hole was centered on the pixel that was directly under the point source. This configuration was chosen because it tends to give intermediate spatial resolution values. For the hexagonal hole geometry described in 4.13 , for example, centering the collimator hole in question directly over a pixel versus centering the hole over the corner of the pixel (where it butts up against three other pixels) gives smaller spatial resolution values over the 0 to $25 \mathrm{~mm}$ imaging distance, and larger spatial resolutions from 30 to $50 \mathrm{~mm}$. In any case, the differences in the spatial resolutions for these two alignments differ by an average of only $3 \%$.

There is some discrepancy between the measured and simulated results for the hexagonal hole collimator. This can be partially accounted for by the fact that the experimental point source was $2 \mathrm{~mm}$ in diameter compared to an infinitesimally small simulated one, and by the existence of a $\sim 1 \mathrm{~cm}$ air gap between the scintillator array and the collimator in the experimental setup but not in simulation (this gap was 
present due to bulky EMF shielding that is miniaturized in the 64-pixel modules). However, simulation of these effects indicates that they account for only $0.3 \mathrm{~mm}$ of the $0.9 \mathrm{~mm}$ spatial resolution difference between experiment and simulation at a $5 \mathrm{~cm}$ imaging distance. The remaining difference may in part be the result of septal penetration and the penetration of gammas through part of one crystal before being absorbed in another crystal, neither of which were included in simulation.

The simulation results of Figure 4.13 suggest that a 1-to-1 matched square hole collimator provides slightly better spatial resolution than a hexagonal one. Comparable sensitivity collimator designs with different hole shapes were chosen by simulating different collimator lengths and counting the percentage of gammas from point sources located at 25 different $\mathrm{x}$, y coordinates that penetrate the collimator holes. This approach accounts for septal absorption in the sensitivity comparison, which is critical for collimators with small hole sizes. The average improvement for the 1-to-1 matched square hole collimator over the hexagonal design across the $0-5 \mathrm{~cm}$ imaging range is $0.32 \mathrm{~mm}$ fwhm. This comes at the expense of a collimator that needs to be quite long $(66 \mathrm{~mm})$ in order to offer the same sensitivity, which is contrary to the goal of compact design. Simulations suggest that using a comparable sensitivity 4-to-1 matched square hole collimator improves the average spatial resolution by an additional $0.08 \mathrm{~mm}$ fwhm and requires a collimator length of only $30 \mathrm{~mm}$. A hexagonal hole collimator with the same sensitivity but a more preferable geometry than the one actually used $(1.0 \mathrm{~mm}$ holes instead of $1.5 \mathrm{~mm}$ holes) would still be shorter at $20 \mathrm{~mm}$. In order to compete with this length, a square hole device would need to implement a 9 -to- 1 matched design with $0.8 \mathrm{~mm}$ holes ( $0.2 \mathrm{~mm}$ septal thickness). This becomes challenging to fabricate with lead microcasting techniques and begins to present sensitivity problems because when looking at the collimator head-on, a large percentage (36\% in this case) of the area is septal wall lead and is therefore "dead" (versus mere air which passes gammas freely).

In simulations the hexagonal hole collimator exhibits more dependence on source location than do either of the square hole collimators. The spatial resolution 
of the hexagonal hole collimator over the 25 source locations demonstrates a standard deviation of $0.47 \mathrm{~mm}$ (this is the average of the individual standard deviations at different imaging distances in the $0-5 \mathrm{~cm}$ range). The standard deviation for both the 1-to-1 and 4-to-1 square hole collimators is $0.45 \mathrm{~mm}$. Despite this very minor difference, there are extreme cases when the spatial resolution of the hexagonal hole collimator is as high as $7.0 \mathrm{~mm}$ fwhm (2.1 mm above the average), while for the square hole collimators the worst resolution was $4.9 \mathrm{~mm}$ fwhm $(0.5 \mathrm{~mm}$ above the average). These deviations from the average spatial resolution result almost entirely from geometric dependencies of the detector array response to point sources located at different $\mathrm{x}, \mathrm{y}$ coordinates. A point source positioned at the border of two pixels, for instance, will tend to split its gammas between those pixels and will demonstrate a wider spatial response than a point source located directly above a single pixel, which will tend to excite that pixel substantially more than any other. There is slightly more deviation in the case of hexagonal hole collimators because the square-hexagon mismatch makes the detector response even more sensitive to source location.

My simulations suggest that the aliasing artifacts which result from shape mismatch between hexagonal collimator holes and square scintillation crystals decrease with decreasing collimator hole diameter. When imaging a point source, these artifacts take the form of select pixels - usually the ones adjacent to the pixel which is directly underneath the point source - collecting an abnormally large number of events and thereby worsening spatial resolution. This occurs because of simple geometric effects involving collimator holes that cover both the central pixel and at least one of the adjacent pixels. An easy way to note the presence of this phenomenon is to observe the dependence of spatial resolution on imaging distance, which under ideal circumstances is a monotonic (and nearly linear) dependency. In the case of this type of aliasing artifact, however, there exists a kink in the dependency where over a short range (approximately $25-35 \mathrm{~mm}$ for the configurations described in this chapter), increasing the imaging distance actually improves the spatial resolution. This again is the result of straightforward geometrical considerations. Over this small range of 
imaging distances the point source is just barely high enough above the collimator that more of the central pixel is exposed to the source than is exposed at shorter imaging distances. The edges of the central pixel which had been obscured by the septal walls of the multiple-pixel collimator holes are just coming out of the septal wall "shadow" as the source rises. As a result, the central pixel collects a larger percentage of the observed events and spatial resolution decreases by as much as $10-15 \%$. For pixel widths in the range of $2-4 \mathrm{~mm}$, this spatial resolution abnormality is no longer readily observable once the hexagonal collimator hole size is less than half the pixel size (Equation 4.15).

Lower limits on the hole diameter are set, however, by manufacturing limitations and by the fact that septal thickness cannot be scaled down along with hole diameter. One major collimator manufacturer (Nuclear Fields, Inc., Des Plaines, IL) offers collimators with hexagonal hole as small as $1.0 \mathrm{~mm}$ in diameter, but only as a custom order which requires advanced tooling and therefore increased expense compared to their standard line of $1.5 \mathrm{~mm}$ hole collimators. The $1.0 \mathrm{~mm}$ designs, which may well become a more standard product line as manufacturing techniques advance, demonstrate promising simulated spatial resolution and sensitivity characteristics with a discrete detector array and are covered thoroughly in Chapter 5.

\subsubsection{System Spatial Resolution with a High Sensitivity Collimator}

An extremely important consideration in collimator design is the classic trade-off between spatial resolution and sensitivity. When imaging in the far field, the sensitivity is roughly proportional to the spatial resolution squared (Equation 1.5), hence a small degradation in the collimator resolution can yield a significant sensitivity improvement. I repeated the measurements described in Section 4.3.4 using a hexagonal hole collimator with nearly twice the sensitivity of the high resolution collimator. This high sensitivity collimator has $2.0 \mathrm{~mm}$ diameter holes, a length of $32 \mathrm{~mm}$, and a sensitivity of 8200 events/mCi/sec. The average observed spatial res- 


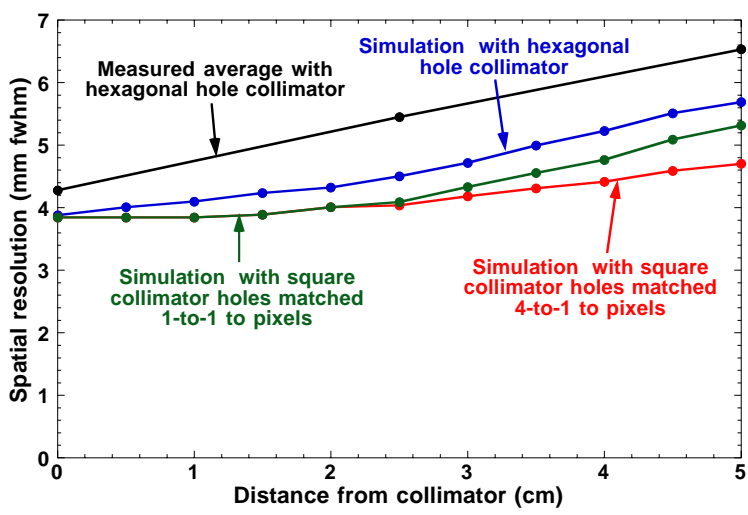

Figure 4.14: Average spatial resolution versus imaging distance for measured and simulated results with high sensitivity collimators. The simulated hexagonal hole collimator has an identical geometry to the experimental collimator, the 1-to-1 matched square hole collimator is $48 \mathrm{~mm}$ long with $2.8 \times 2.8 \mathrm{~mm}^{2}$ holes $(0.2 \mathrm{~mm}$ septal thickness makes unit cells $3.0 \times 3.0 \mathrm{~mm}^{2}$ ), and the 4 -to- 1 matched square hole collimator is $21 \mathrm{~mm}$ long with $1.3 \times 1.3 \mathrm{~mm}^{2}$ holes $(0.2 \mathrm{~mm}$ septal thickness makes unit cells $\left.1.5 \times 1.5 \mathrm{~mm}^{2}\right)$. All collimators have sensitivities of about 8200 events $/ \mathrm{mCi} / \mathrm{sec}$.

olution is $4.3 \mathrm{~mm}$ fwhm at a $0.0 \mathrm{~cm}$ imaging distance, $5.5 \mathrm{~mm}$ fwhm at $2.5 \mathrm{~cm}$, and $6.5 \mathrm{~mm}$ fwhm at $5.0 \mathrm{~cm}$. Observed and simulated spatial resolutions for this collimator, as well as simulations for 1-to-1 and 4-to-1 matched square hole collimators of comparable sensitivity, are shown in Figure 4.14.

When using the high sensitivity hexagonal hole collimator, the measured spatial resolution degrades an average of $0.50 \mathrm{~mm}$ fwhm compared to the high resolution collimator. The associated doubling in sensitivity, however, would decrease patient dose, decrease imaging time, and/or improve counting statistics. The high sensitivity hexagonal hole collimator demonstrates a worse spatial resolution in measurements than in simulation for the same reasons discussed in Section 4.3.4.

The simulated 1-to-1 matched square hole collimator (48 mm long) demonstrates an average spatial resolution improvement of $0.31 \mathrm{~mm}$ fwhm compared to the simulated hexagonal hole collimator, while the 4-to-1 square hole collimator $(21 \mathrm{~mm}$ 
long) exhibits a further improvement of $0.17 \mathrm{~mm}$ fwhm. As with the high resolution collimators, a high sensitivity hexagonal hole collimator with $1.0 \mathrm{~mm}$ holes would be shortest at $15 \mathrm{~mm}$.

The standard deviation of the spatial resolution demonstrated by the simulated hexagonal hole collimator averages $0.49 \mathrm{~mm}$, compared to $0.37 \mathrm{~mm}$ and $0.38 \mathrm{~mm}$ for the 1-to-1 and 4-to-1 square hole collimators, respectively. A few extreme cases are evident - the maximum spatial resolution of the hexagonal hole collimator is $8.5 \mathrm{~mm}$ fwhm (2.8 $\mathrm{mm}$ above average), whereas the worst resolution exhibited by either square hole collimator is $5.9 \mathrm{~mm}$ fwhm (0.6 $\mathrm{mm}$ above average).

\subsection{Summary and Analysis}

The prototype $3 \times 4$ pixel discrete scintillation camera module I constructed demonstrates good energy and spatial resolution characteristics that suggest a full camera consisting of an array of modules would prove a successful scintimammography imaging device. An average energy resolution of $10.7 \%$ fwhm was demonstrated for ${ }^{99 m} \mathrm{Tc}$, and observations by other researchers indicate that better $\mathrm{CsI}(\mathrm{Tl})$ crystals and cooled electronics can yield an energy resolution as low as $7.5 \% \mathrm{fwhm}$. There is some hope that this discrete scintillation camera technology can meet or even surpass the $\sim 9 \%$ fwhm room temperature energy resolution typically demonstrated by conventional scintillation cameras. Certainly the prototype module can be considered a successful proof-of-concept for the use of low-noise Si PIN photodiode arrays and compact readout ASICs in discrete $\mathrm{CsI}(\mathrm{Tl})$ scintillation cameras.

The spatial resolution I observed when using a high resolution hexagonal hole collimator (4300 events $/ \mathrm{mCi} / \mathrm{sec}$ ) is $5.9 \mathrm{~mm}$ fwhm at $5 \mathrm{~cm}$ (near the anticipated maximum tumor-to-camera imaging distance), and simulations suggest the possibility of improving this performance. This good spatial resolution is notable because scintimammography with conventional scintillation cameras is poor at detecting tumors less than $1 \mathrm{~cm}$ in diameter. This advanced compact gamma camera technology 
may prove capable of seeing significantly smaller lesions.

Collimator design has a crucial impact on camera performance. With hexagonal hole collimators, increasing the sensitivity from 4300 to 8200 events $/ \mathrm{mCi} / \mathrm{sec}$ degrades the spatial resolution by only about $0.5 \mathrm{~mm}$ fwhm over the $0-5 \mathrm{~cm}$ imaging range, from $4.1-5.9 \mathrm{~mm}$ fwhm to $4.3-6.5 \mathrm{~mm}$ fwhm. Simulations suggest that the choice of a 1-to-1 matched square hole collimator over a hexagonal one of comparable sensitivity improves spatial resolution by an average of about $0.4 \mathrm{~mm}$ fwhm, but at the cost of a longer, less compact collimator. A 4-to-1 matched square hole collimator further improves spatial resolution by about $0.1 \mathrm{~mm}$ and allows a reasonably short collimator. The spatial resolution of both square hole collimator configurations demonstrate less dependence on source location than does that of a comparable sensitivity hexagonal hole collimator. The hexagonal collimator should have a more uniform septal penetration pattern, but this effect was not included in the simulations.

The advantages and disadvantages inherent in the choice between hexagonal and matched square hole collimators, however, will have a much smaller impact on the ability of a camera to detect breast lesions than will the traditional trade-off between spatial resolution and sensitivity. This optimization is heavily dependent on assumptions regarding lesion sizes, relative tracer uptake ratios, imaging distances, and imaging time, and is addressed in the following chapter. 


\section{Chapter 5}

\section{Monte Carlo Simulations of Breast Tumor Imaging Characteristics}

In theory, there is no difference between theory and practice. But, in practice, there is.

—Jan L.A. van de Snepscheut

In this chapter I describe my Monte Carlo simulation results for breast tumor imaging using a compact, discrete gamma camera. This includes the CsI(Tl) scintillator/Si PIN photodiode technology described in this thesis but applies equally well to any pixellated cameras (including CdZnTe designs). Despite the fact that the performance of both types of gamma camera is strongly influenced by camera geometry (including both collimator configuration and pixel size), prior to the work described in this chapter and reference [95] there had been no comprehensive exploration of how the configuration of these cameras affects breast tumor imaging. Simulations to date have generally dealt with either traditional Anger cameras $[125,126,127,128]$ or compact cameras utilizing a PSPMT [129]. Those that have 
analyzed discrete gamma camera geometries have looked at imaging a simple point source in the absence of background activity, much like the simulations presented in Chapter $4[93,96]$. The Monte Carlo code discussed herein is designed to be more representative of the taking of a breast tumor image in a realistic scintimammography situation.

I designed the Monte Carlo simulations to analyze and optimize camera design for the final 64-pixel CsI(Tl)/Si photodiode modules, particularly as pertains to collimator configuration and detector pixel size. Simulated planar images of 5-15 mm diameter tumors in a 3-D phantom patient (including a breast, torso, and heart) were generated for imaging distances of $5-55 \mathrm{~mm}$, pixel sizes of $2 \times 2$ to $4 \times 4 \mathrm{~mm}^{2}$, hexagonal and square hole collimators, and collimator sensitivities ranging from 4000 to 16,000 counts $/ \mathrm{mCi} / \mathrm{sec}$. Other factors considered include T/B (tumor-to-background tissue uptake ratio) and detector energy resolution. Image properties were quantified by computing the observed tumor fwhm and $\mathrm{S} / \mathrm{N}$ (sum of detected tumor events divided by the statistical noise). Results suggest that hexagonal and square hole collimators perform almost comparably, that higher sensitivity collimators provide higher tumor $\mathrm{S} / \mathrm{N}$ with little increase in the observed tumor fwhm, that smaller pixels only slightly improve tumor fwhm and S/N, and that improved detector energy resolution has little impact on either the observed tumor fwhm or the observed tumor $\mathrm{S} / \mathrm{N}$.

\subsection{Simulation Methods}

Recall the basic components of a compact, discrete scintillation camera from Figure 1.4: a collimator, a discrete scintillator array, a photodiode array, and readout electronics. A CdZnTe camera is similar in concept, with the scintillator crystals and photodiode array replaced by a solid-state CdZnTe detector array. The simulation program was written primarily as a tool to study geometric effects related to the collimator and pixellated detector array (either scintillator/photodiode or CdZnTe) 
when such a camera is used in practical imaging situations. It therefore includes background events from non-specific radionuclide uptake, photoelectric absorption, and Compton scatter. Gamma rays - both from the tumor and from healthy tissueare generated in a 3-D phantom patient (Figure 5.1) and the code tracks the flight paths of the gammas through the patient and the collimator and determines which camera pixel, if any, detects each event. A breast thickness of $60 \mathrm{~mm}$ was chosen based on the assumption that the breast would be moderately compressed. The reasons for compressing the breast during a scintimammography study include:

- It immobilizes the breast and minimizes motion artifacts.

- It brings tissue closer to the camera where the spatial resolution is finer.

- It provides a more uniform background in the resulting image by presenting the entire camera field of view (FOV) with a similar depth of tissue (and hence similar quantity of radionuclide).

The simulation includes both Compton scattering and photoelectric absorption within the phantom patient. It fully implements collimator geometry, including hole diameter, hole shape (hexagonal or square), channel length, and finite septal thickness, and the exact collimator configurations that were simulated are described throughout this chapter. The program, however, assumes that no photons penetrate the collimator septa and does not model either scatter or lead X-ray fluorescence resulting from gamma interactions in the collimator itself. Ideally these phenomena would be implemented, and they were omitted solely because the majority of the code development effort was spent on what were deemed to be more important issues. Detector count rate limitations (pulse pileup, etc.) were also omitted, but this is a reasonable assumption because of the low count rates $(<0.5 \mathrm{~Hz} /$ pixel $)$ encountered in the simulated scintimammography studies. Detector energy resolution is included by convolving the true energy with a Gaussian whose width is proportional to the square root of the energy. The energy resolution is easily adjusted, but for most of 


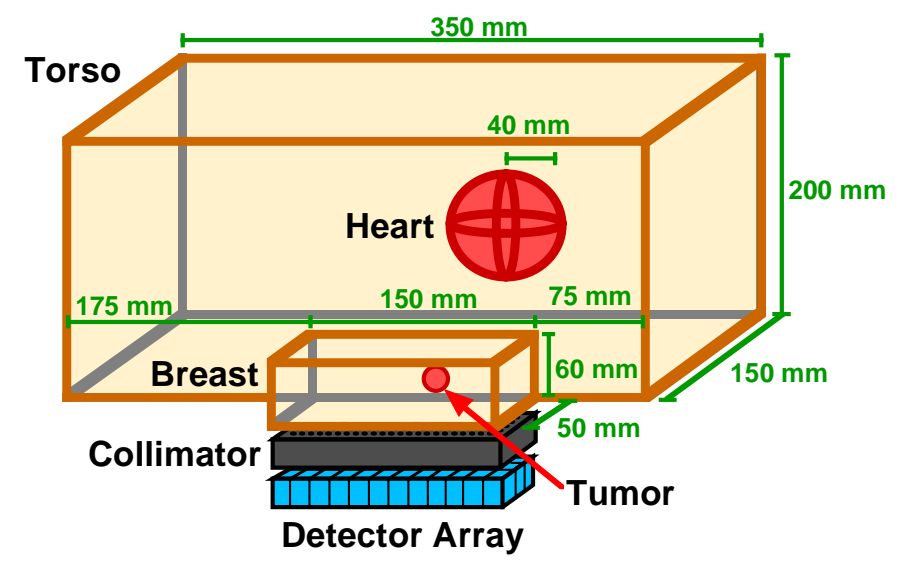

Figure 5.1: 3-D phantom and camera positioning used for Monte Carlo simulations designed to emulate scintimammography. The simple, primarily rectangular geometry greatly speeds computational time while permitting the study of a number of realistic phenomenon including non-specific radionuclide uptake, attenuation, and scatter.

the simulations it was set to $10 \%$ fwhm. Detector pixels have no dead space between them, and it is assumed gamma rays deposit all their energy in the first pixel they encounter. Finally, the energy of the simulated gammas is easily adjusted but was set to $140 \mathrm{keV}$ for all of the simulations presented in this chapter.

The random number generation and associated routines (e.g., photon ray tracing to determine scatter and absorption of individual gammas) have been developed at Lawrence Berkeley national Laboratory over the years with the coding described in [130] as a starting point. The specific routines used implemented in this Monte Carlo code are most recently described in [131].

\subsubsection{Verification of Simulation Code}

I have validated the entire Monte Carlo program by verifying the accurate implementation of its simulated geometries and by histogramming various results for comparison against the appropriate theoretical distributions. Generally the valida- 
tion consisted of looking carefully at the performance of individual sections of the program, which can be loosely categorized as:

- generation of background photons throughout the phantom patient

- Compton scatter and photoelectric absorption of photons travelling through the phantom

- collimator absorption or acceptance of individual photons

- calculation of which pixel detects a photon that successfully penetrates the collimator

- simulation of finite detector energy resolution and energy discrimination (photon acceptance or rejection)

- analysis of the simulated image, including calculation of tumor fwhm and S/N.

The methods used to verify the proper operation of these individual sections, along with the observed results, follow below.

The generation of background photons was validated by histogramming the location and direction vectors of simulated photons. As desired, (1) they are uniformly distributed throughout the phantom body sections and (2) they have isotropic direction vectors. Further, the number of photons generated in each body section (tumor, breast, torso, and heart) was counted to verify that: (1) the photons/volume is the same in the breast and torso, (2) the photons/volume in the heart is 10 times greater than in the breast and torso, and (3) the photons/volume in the tumor is correctly set and adjusted by the $\mathrm{T} / \mathrm{B}$ value.

The simulated interaction of gamma rays with matter (i.e., the phantom patient) was validated by tracking individual photons through the Compton scattering and photoelectric absorption processes. Photon path lengths (distance traversed before scattering or absorption) were histogrammed to ensure that proper exponential attenuation is realized. Because attenuation is energy-dependent, this check was 


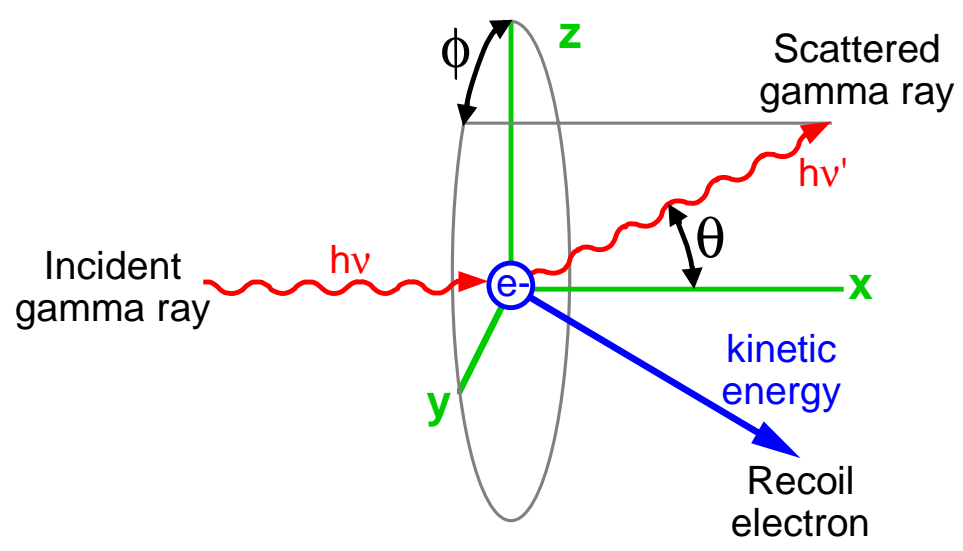

Figure 5.2: The angular deflections that result during Compton scatter between a gamma ray and a free electron.

performed at a number of different photon energies ranging from 51 to $511 \mathrm{keV}$ (the Monte Carlo program implements energy dependence by retrieving the Compton and photoelectric attenuation coefficients from a look-up table based on the energy of the photon being analyzed). As to the Compton scatter of photons (Figure 5.2), the $\phi$ angles (the $2 \pi$ range that defines the direction in which the photon was scattered relative to its initial direction vector) of scattered photons were histogrammed to confirm that they are uniform over $2 \pi$ radians, while the $\theta$ angles (the angular difference between the pre and post-scatter gamma direction vectors) were histogrammed to verify that the resulting distribution is consistent with the Klein-Nishina formula. The differential cross section per electron per solid angle, $\mathrm{d} \Omega$, given by the KleinNishina formula is:

$$
\begin{aligned}
\frac{d \sigma_{c}^{e}}{d \Omega}= & \frac{e^{4}}{2 m_{o}^{2} c^{4}}\left(1+\cos ^{2} \theta\right)\left(\frac{1}{1+\alpha(1-\cos \theta)}\right)^{2} \\
& \times\left(1+\frac{\alpha(1-\cos \theta)^{2}}{[1+\alpha(1-\cos \theta)]\left(1+\cos ^{2} \theta\right)}\right)
\end{aligned}
$$

where $\alpha=h \nu$, the incident gamma ray energy. Finally, the number of Compton scatter events relative to the number of photoelectric absorption events were counted 
to verify that this proportion is consistent with the Compton and photoelectric crosssections. These checks were also performed at a number of different photon energies. I had previously implemented and validated collimator geometry (the acceptance and rejection of incident gammas) in the preliminary Monte Carlo code from Chapter 4. Photons were individually tracked "by hand" to verify that, assuming a straight line path, the coordinates of the photons at the front face and the back face of the collimator were correct and that the program correctly determines whether each photon is absorbed by the collimator (i.e., whether its straight line path ever passes into lead). Other tests that were also performed included moving a simulated point source around the front face of the collimator to verify that: (1) when the source is located directly on top of a septal wall $(0 \mathrm{~mm}$ from the collimator), no photons penetrate the collimator and (2) when the source is located directly on top of a collimator hole ( $0 \mathrm{~mm}$ from the collimator), photons penetrate that hole and only that hole. The pattern of collimator holes showing any nonzero penetration from photons originating at a point source various distances from the collimator face was also analyzed and compared against analytic calculations. All of these verifications were performed for multiple collimator designs: (a) both square and hexagonal holes, (b) varying channel lengths (down to $0 \mathrm{~mm}$ ), (c) varying septal thicknesses (down to $0 \mathrm{~mm}$ ), and (d) varying hole diameters (down to $0 \mathrm{~mm}$ ).

Determination of which pixel detects a photon that penetrates the collimator was also previously implemented and validated in the preliminary Monte Carlo code from Chapter 4. This check was a simple matter of looking at the coordinates of individual photons and confirming that they are counted in the correct pixels.

Finite energy resolution was validated by histogramming the simulated detected energy of monoenergetic photons and verifying that the resulting distribution is a Gaussian centered at the true photon energy and demonstrating the expected fwhm value of 10\% (since 10\% fwhm energy resolution was implemented). Histogramming of the detected photon energies was also performed after energy discrimination to verify that all photons with energies within the acceptance window are accepted 
and that all other photons are rejected. This verification was performed for a number of different photon energies, detector energy resolutions, and energy acceptance windows.

Finally, the calculation of observed tumor properties, namely spatial fwhm and S/N (see Section 5.1.5), was validated by taking the raw image data (i.e., counts per pixel), calculating the values "by hand," and comparing the results with those of the Monte Carlo program. This was a very simple task requiring, in the case of spatial fwhm, looking at the row and the column with the greatest intensity of response and estimating the fwhm of the two waveforms using simple linear interpolation and averaging the results. Similarly, I counted tumor-generated detected events and background detected events (the simulation code makes this distinction) over the appropriate area in the simulated image and calculated $\mathrm{S} / \mathrm{N}$ values in the manner described in Section 5.1.5 and Equation 5.3. Intermediate values critical to the calculation of both the spatial fwhm (such as the amplitude considered to be half the $\max$ ) and the $\mathrm{S} / \mathrm{N}$ (such as the number tumor-generated detected events in a given pixel area) were also compared. As an example, for a $10 \mathrm{~mm}$ tumor with a $\mathrm{T} / \mathrm{B}$ of 10 imaged at a distance of $25 \mathrm{~mm}$ with a high sensitivity hexagonal hole collimator, the code computes a spatial fwhm of $7.10 \mathrm{~mm}(6.72 \mathrm{~mm}$ in $x$ and $7.49 \mathrm{~mm}$ in $y)$ and a $\mathrm{S} / \mathrm{N}$ of 25.4. Analyzing the image data myself, I calculated a spatial fwhm of $7.21 \mathrm{~mm}(6.81 \mathrm{~mm}$ in $x$ and $7.60 \mathrm{~mm}$ in $y)$ and a $\mathrm{S} / \mathrm{N}$ of 25.4. In general the spatial resolution differed by no more than $0.5 \mathrm{~mm}$ (because of the error inherent in the linear interpolation used in the "by hand" calculations), while the $\mathrm{S} / \mathrm{N}$ values were always exactly the same. These verifications were performed for a range of tumor sizes, T/B (tumor-to-background) uptake ratios, and tumor-to-collimator distances appropriate to the simulations described in this chapter. 


\subsubsection{Background Activity and Imaging Time}

In order to emulate a clinical scintimammography scan the number of simulated gamma rays must be commensurate with the typical imaging time of 10 minutes. Unfortunately, there is a significant lack of hard data in the available literature regarding the activity densities that exist in patient tissues during scintimammography scans. A background activity density of $80 \mathrm{nCi} / \mathrm{cm}^{3}$ is believed to be reasonable [132] and is assumed for the breast and torso phantom sections. The heart is known to have a significantly higher activity density than most other non-tumor tissues, largely due to its substantial blood supply and well-developed vasculature. Because of such strong uptake in the heart, for example, ${ }^{99 m}$ Tc-Sestamibi was originally developed as a heart perfusion agent. A heart activity density of $800 \mathrm{nCi} / \mathrm{cm}^{3}$, or 10 times that of the breast and torso, is assumed because it represents a very substantial uptake (equal to the upper bound of tumor uptake) and should make the heart a very prominent feature when in or near the field of view. The exact activity of the heart is not as important as is making it an important feature whose influence must be reckoned with in the scintimammography imaging situation. Tumor activity density in the simulations is variable, and is equal to the $\mathrm{T} / \mathrm{B}$ ratio multiplied by the torso and breast activity density of $80 \mathrm{nCi} / \mathrm{cm}^{3}$.

Given the heart volume of $268 \mathrm{~cm}^{3}$ and total phantom volume of $10,950 \mathrm{~cm}^{3}$, the total phantom activity is about $1.07 \mathrm{mCi}$. By contrast, the total activity injected into a patient during a scintimammography study is typically $20 \mathrm{mCi}$. Hence the total activity assumed in the phantom patient is nearly 19 times less than the total activity injected in a real study. Even after adjusting for omissions in the phantom geometry (e.g., it has no legs, head, or bladder), the total activity in the phantom patient is reasonably conservative compared to the $20 \mathrm{mCi}$ benchmark. This then suggests that the background activity estimate of $80 \mathrm{nCi} / \mathrm{cm}^{3}$ is also reasonably conservative. The total phantom activity of $1.07 \mathrm{mCi}$, or $3.96 \times 10^{7} \mathrm{~Bq}$, corresponds to $2.38 \times 10^{10}$ disintigrations over a period of 10 minutes, and therefore that number 
of gamma rays must be analyzed for each simulated scan. In all cases $140 \mathrm{keV}$ gamma rays from ${ }^{99 m} \mathrm{Tc}$ were simulated and, except where otherwise noted, the detector energy resolution was assumed to be $10 \%$ fwhm with an energy acceptance window of $126-154 \mathrm{keV}$.

\subsubsection{Generation of Background Events}

When imaging a breast tumor the photons originating in the lesion are obviously of greatest interest, yet they represent fewer than $0.1 \%$ of all emitted gammas. Thus the vast majority of computational time is spent generating background photons (those from the breast, torso, or heart). This dilemma is particularly severe for the torso and heart, which produce only $10 \%$ and $3 \%$, respectively, of the background counts in the final planar image (as compared to $87 \%$ for the breast).

In order to decrease the computational time spent simulating background gammas in many imaging scans with virtually identical phantom geometries, a large pool of $1.6 \times 10^{11}$ background photons was generated and analyzed. The energy, position, and direction of the $\sim 6 \times 10^{7}$ gammas that successfully reached the front face of the collimator with energy $\geq 80 \mathrm{keV}$ and with an angle to normal $\leq 11^{\circ}$ (over twice the acceptance angle of the highest sensitivity collimators used) were saved. About $4 \times 10^{7}$ of these photons originated within the breast, and for each simulated scan $\sim 6 \times 10^{6}$ breast photons are randomly selected from this pool. Meanwhile, algorithms mimicking the energy, direction, and position distributions observed for torso and heart photons are used to quickly generate torso and heart gammas that impinge upon the front face of the collimator. The simulation of the specific collimator and detector geometries is performed for every single one of these pre-generated background photons. Taken as a whole, these time-saving efforts maintain random background variations from scan to scan and result in negligible changes to (or biasing of) the background. For instance, there is no apparent difference between histograms of the energy, direction, and position of background photons generated 
using these time-saving methods compared with histograms of background photons from simulations that do not.

\subsubsection{The Rose Equation and Tumor Detectability}

The traditional model which quantifies the visibility of a simple feature obscured by a noisy background (e.g., a tumor visualized in a nuclear medicine planar image) is the Rose equation [133]:

$$
\text { Number of counts in image }=\frac{\text { Area }_{\text {total }}}{\text { Area }_{\text {feature }} C^{2}} k^{2}
$$

where $C$ is the contrast between the feature and the background and $k$ is the confidence level that the feature under observation is truly there and is not merely a false alarm. This equation therefore defines the number of events that must be collected in an image with area $A r e a_{t o t a l}$ in order to correctly identify (with the confidence level set by $k$ ) a feature with contrast $C$ and area Area feature. The contrast, $C$, ranges from 0 to 1 and is equal to $\Delta B / B$, where $B$ is the photon intensity (in events/area) in the feature area, and $B-\Delta B$ is the photon intensity (in events/area) in the background portion of the image which surrounds the feature. The confidence level, $k$, determines the probability that a feature will be incorrectly identified because of random fluctuations, a probability which is defined by the area under a Gaussian curve over the range from $x=k \sigma$ to $x=\infty$. Table 5.1 presents the probability of this type of incorrect identification occurring for various possible $k$ values.

Equation 5.2 assumes that the photon intensities across the feature area (i.e., $B$ ) and the image background (i.e., $B-\Delta B$ ) are not spatially variant and are essentially constant. While this is not an ideal representation of a tumor projection on a planar image (the tumor feature will resemble a Gaussian much more than a rectangle), Equation 5.2 nonetheless provides a very useful first order analysis of this visualization problem. 


\begin{tabular}{|c|c|}
\hline Confidence level, $k$ & Probability of a false alarm \\
\hline 0 & 0.5 \\
1 & 0.15 \\
2 & 0.023 \\
3 & $1.3 \times 10^{-3}$ \\
4 & $3 \times 10^{-5}$ \\
5 & $3 \times 10^{-7}$ \\
6 & $2 \times 10^{-9}$ \\
\hline
\end{tabular}

Table 5.1: Probability that an identified feature is a false alarm for several values of $k$, which sets the level of confidence. Generally $k=5$ is considered an acceptably high level of confidence.

\subsubsection{Characterizing Tumor Images}

In order to compare tumor images from different scans, two values are quantified: observed tumor spatial fwhm and observed tumor S/N. The tumor spatial fwhm is calculated as the fwhm of a curve fit to the number of tumor events detected in a row of pixels. The curve fitting is performed with a series of sinc functions $\left(\frac{\sin (x)}{x}\right)$, ensuring that the value of the fit curve exactly matches the discrete data points while providing a smooth transition between them. Values reported in this chapter are the average of four such fwhm values for each tumor-one along each of the $x, y$, and two $45^{\circ}$ diagonal directions. Because the curve fitting requires the interpolated curve to exactly match the discrete data points, statistical variations tend to make calculated tumor fwhm values slightly smaller than the ideal for a projection image of a sphere (i.e., $87 \%$ of the diameter). Tumor spatial fwhm is of interest because it reveals how much the spatial resolution of the camera spreads out the tumor dimensions in the planar image, an effect which, if severe enough, will tend to obscure small features and decrease image quality.

Applying the basic premises in Section 5.1.4, observed tumor $\mathrm{S} / \mathrm{N}$ is calcu- 
lated as:

$$
S / N=\frac{\sum_{\text {pixels in tumor ROI }} \text { detected tumor events }}{\sqrt{\sum_{\text {pixels in tumor ROI }} \text { all detected events }}}
$$

where the pixels chosen as the tumor ROI (region of interest) are a symmetric pattern of $1,5,9,13,21,29,37$, or 45 pixels centered beneath the tumor. For every simulated image the $\mathrm{S} / \mathrm{N}$ is computed using each of these possible ROIs and the maximum $\mathrm{S} / \mathrm{N}$ value is reported. Note that the numerator of this equation represents the summation of all photons generated by the tumor which were detected by the camera within the ROI, whether or not the gammas scattered along the way. Conversely, the denominator represents all photons detected within the ROI regardless of where they were generated. The Monte Carlo code has the advantage over real world imaging situations of being able to keep track of which body section (Figure 5.1) generated each event, and it exploits that capability in the S/N calculation. Since tumor visibility estimates like Equation 5.2 rely upon analysis of statistical counting errors which generate random deviations from mean values for the number of detected photons, it is helpful to have very accurate mean values in the first place. Making the distinction between tumor-generated events and background events when calculating the $\mathrm{S} / \mathrm{N}$ as per Equation 5.3 helps to ensure the accuracy of the numerator (the signal). Otherwise this value would have to calculated as the difference between the total number of events in the tumor ROI (background events plus tumor events) and the total number of events in an equal number of pixels outside of the ROI (background events), and it would therefore be subject to a greater level of statistical error. Such error makes trends more difficult to discern, which is contrary to the purpose of these simulations.

The $\mathrm{S} / \mathrm{N}$ value is meant to quantify how visible a tumor would be against the local background and thus allow for the comparison of images taken with different camera configurations. Equation 5.3 has been based on Equation 5.2 such that $\mathrm{S} / \mathrm{N}$ approximates $k$, the confidence level for the detection of the tumor. As a result, 
a higher $\mathrm{S} / \mathrm{N}$ indicates an increased likelihood that the tumor can be successfully identified. Since the summation of detected tumor events in the ROI (the numerator of Equation 5.3) is analogous to $\Delta B \times$ Area $_{\text {feature }}$ and the summation of all events in the ROI (the square of the denominator in Equation 5.3) is analogous to $B \times$ Area $_{\text {feature }}, \mathrm{S} / \mathrm{N}$ can be expressed as:

$$
S / N=\frac{\Delta B \text { Area } a_{\text {feature }}}{\sqrt{B \text { Area } a_{\text {feature }}}}
$$

which can be further rearranged into:

$$
S / N=\frac{\Delta B}{B} \sqrt{\text { Area }_{\text {feature }}} \sqrt{B} .
$$

Rearranging Equation 5.2 into a like form yields:

$$
k=C \sqrt{\text { Area }_{\text {feature }}} \sqrt{\frac{\text { Number of counts in image }}{\text { Area }} . \text { total }} .
$$

Thus, since $C=\frac{\Delta B}{B}$ and $B \approx \frac{\text { Number of counts in image }}{\text { Area }_{\text {total }}}, \mathrm{S} / \mathrm{N} \approx k . \mathrm{S} / \mathrm{N}$ therefore provides an estimate for tumor visibility, but obviously an ROC (receiver operator characteristic) study would be necessary to quantify true tumor detectability.

\subsubsection{Expected Tumor Event Rate}

Clinical scintimammography studies with traditional Anger cameras have generally shown decreased sensitivity for tumors less than $10 \mathrm{~mm}$ in diameter [60,61], in part due to the small volume of the tumor and hence low total activity in the lesion. Table 5.2 displays the expected number of detected tumor-generated gamma rays for a 10 minute scan with collimators of various sensitivities and tumors of various sizes (each with a $\mathrm{T} / \mathrm{B}$ of 5 ). These are best case values in that no attenuation has been assumed - real tumors at any finite depth in the breast will tend to produce an even lower number of detected gammas. Theses estimates for the number of tumorgenerated events do not by themselves indicate actual tumor detectability, but rather they provide a glimpse into the size of the planar image "signal" (the numerator in 


\begin{tabular}{|c|c|c|c|}
\hline $\begin{array}{c}\text { Collimator } \\
\text { sensitivity }\end{array}$ & $\begin{array}{c}\text { High resolution } \\
(4000 \mathrm{cps} / \mathrm{mCi})\end{array}$ & $\begin{array}{c}\text { All purpose } \\
(8000 \mathrm{cps} / \mathrm{mCi})\end{array}$ & $\begin{array}{c}\text { High sensitivity } \\
(16,000 \mathrm{cps} / \mathrm{mCi})\end{array}$ \\
\hline & \multicolumn{2}{|c|}{ Number of tumor events detected in $10 \mathrm{~min}$} \\
\hline $5 \mathrm{~mm}$ tumor & 63 & 125 & 250 \\
$7.5 \mathrm{~mm}$ tumor & 210 & 420 & 850 \\
$10 \mathrm{~mm}$ tumor & 500 & 1000 & 2000 \\
$15 \mathrm{~mm}$ tumor & 1700 & 3400 & 6700 \\
\hline
\end{tabular}

Table 5.2: Expected number of tumor events detected during a 10 minute scan for different collimator sensitivities and tumor sizes, assuming a $\mathrm{T} / \mathrm{B}$ of 5 (i.e., tumor activity density $=400 \mathrm{nCi} / \mathrm{cm}^{3}$ ). These are best case values in that no attenuation due to Compton scatter or photoelectric absorption has been factored in.

Equation 5.3) that must be recognized against the noisy background in order to successfully identify a breast tumor. The limited number of tumor events available from a $5 \mathrm{~mm}$ tumor, for instance, immediately suggests that it will be very challenging to detect (unless the background is not very noisy). The simulations in this chapter focus on tumors $7.5 \mathrm{~mm}$ or larger in diameter because they are more likely to be detectable and still represent an improvement over the current scintimammography capabilities.

\subsection{Simulation Results}

\subsubsection{Typical Tumor Images and Background Patterns}

Four examples of tumor images generated by the simulation code with an optimistic T/B of 10 are presented in Figure 5.3, illustrating the role of tumor size and depth. A $7.5 \mathrm{~mm}$ diameter tumor close to the collimator (a) has a fwhm of $5.9 \mathrm{~mm}$ and a $\mathrm{S} / \mathrm{N}$ of 17.5 , while at greater depth the same tumor (b) has a similar fwhm of $6.1 \mathrm{~mm}$ but a $\mathrm{S} / \mathrm{N}$ of only 7.5, making the tumor difficult to discern. A $15 \mathrm{~mm}$ diameter tumor close to the collimator (c) has a fwhm of $10.7 \mathrm{~mm}$ and a $\mathrm{S} / \mathrm{N}$ of 62.8 , 
while at greater depth (d) its fwhm is about the same at $11.4 \mathrm{~mm}$ but its $\mathrm{S} / \mathrm{N}$ drops significantly to 37.2. Figure 5.4 illustrates a typical background pattern for these images (i.e., the pattern of counted gammas that originated from non-tumor tissue, namely the breast, torso, and heart). It has an average of 164 counts/pixel with a standard deviation of 17.9 counts/pixel and demonstrates a slight gradient - there are an average of 194 counts/pixel next to the chest wall and only 150 counts/pixel farthest from it.

\subsubsection{Collimator Sensitivity and Hole Shape}

Compact, discrete gamma camera technology is not immune to the dependence of single photon imaging on the classic trade-off between collimator spatial resolution and sensitivity. The desire to see small tumors $\leq 10 \mathrm{~mm}$ in diameter suggests emphasizing high resolution, while the count-limited situation described in Table 5.2 suggests emphasizing high sensitivity. Additionally, a choice must be made between hexagonal or square collimator holes. Hexagonal hole collimators are more readily available from industry and have a more symmetric septal penetration pattern, but square holes can be matched 1-to-1 or 4-to-1 to the square detector pixels and may provide a superior system point spread response with minimal dependence on source position by eliminating the aliasing due to geometric mismatch between hexagonal collimator holes and square detector pixels (Figure 5.5). Previous simulations with hexagonal hole collimators (see Section 4.3.4 and Equation 4.15), however, suggest that while this type of aliasing manifests itself in the form of a point source spatial resolution that displays non-monotonic dependence on the imaging distance, the aliasing problem is small and not readily observable with a point source if the collimator hole size is less than about half the pixel size.

Figures 5.6 and 5.7 display simulation results for the observed tumor fwhm and $\mathrm{S} / \mathrm{N}$, respectively, for a $10 \mathrm{~mm}$ diameter tumor imaged with six different collimator designs. Hexagonal hole collimators have channels $1.0 \mathrm{~mm}$ in diameter and 21, 15, 


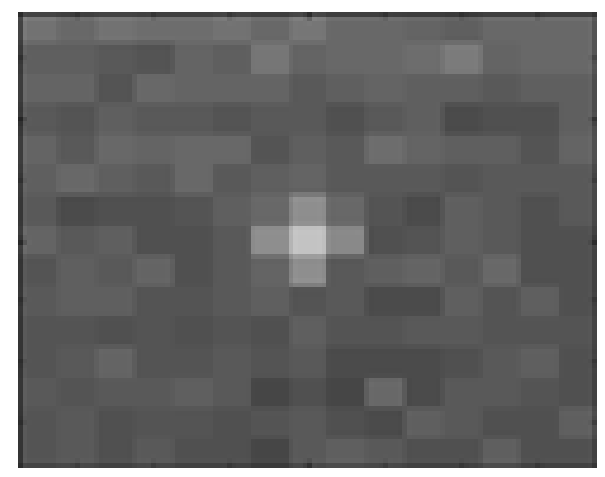

(a)

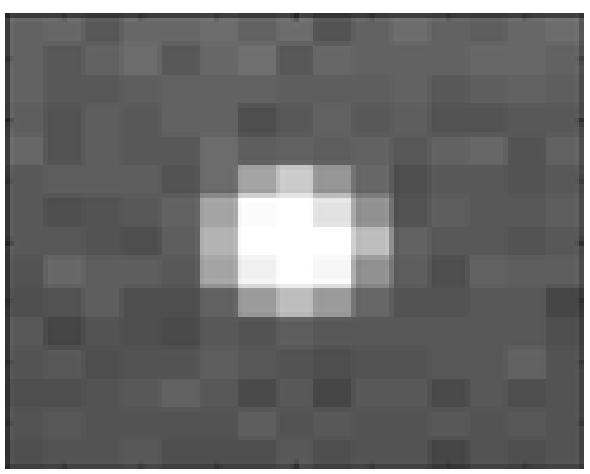

(c)

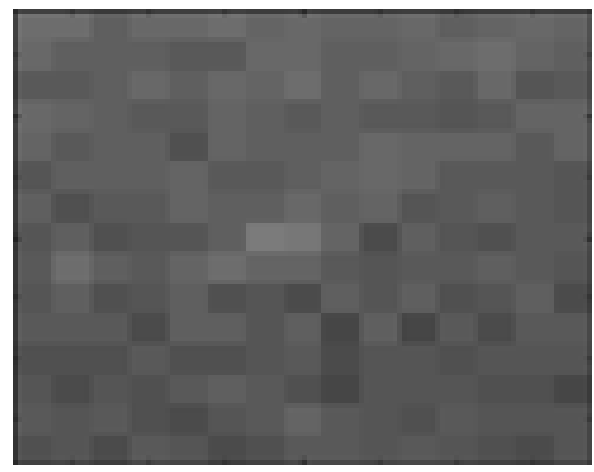

(b)

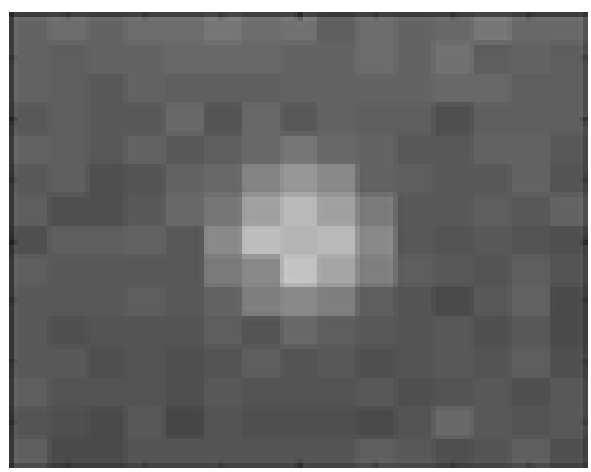

(d)

Figure 5.3: Typical images produced by the Monte Carlo simulation. A $7.5 \mathrm{~mm}$ diameter tumor (a) $5 \mathrm{~mm}$ and (b) $55 \mathrm{~mm}$ deep, and a $15 \mathrm{~mm}$ diameter tumor (c) $7.5 \mathrm{~mm}$ and (d) $52.5 \mathrm{~mm}$ deep. No gray scale enhancement or windowing has been performed. All four images use a 8000 counts $/ \mathrm{mCi} / \mathrm{sec}$ sensitivity hexagonal hole collimator, $3 \times 3 \mathrm{~mm}^{3}$ pixels, a T/B of 10 , and the same gray scale. The tops of the images are closest to the chest wall. 


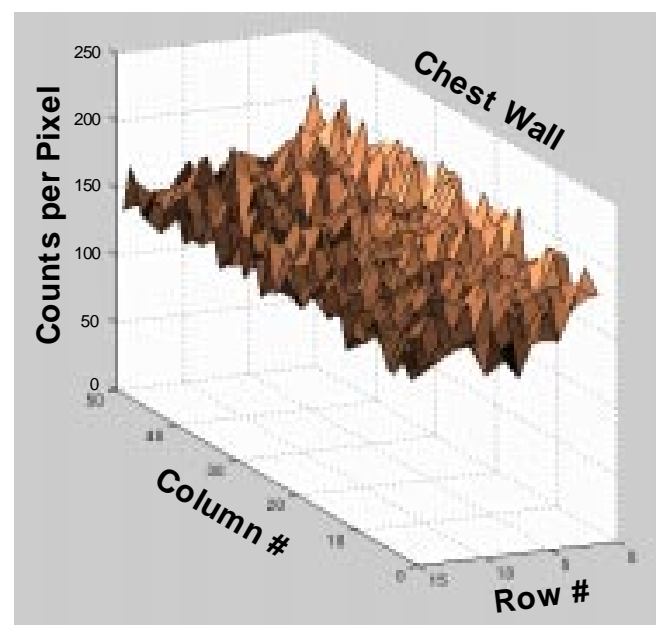

Figure 5.4: Typical Monte Carlo background pattern produced by gammas from the breast, torso, and heart of the phantom patient. The collimator has hexagonal holes and a 8000 counts $/ \mathrm{mCi} / \mathrm{sec}$ sensitivity, while the pixel size is $3 \times 3 \mathrm{~mm}^{2}$. There are an average of 164 counts/pixel with a standard deviation of 17.9 counts/pixel.

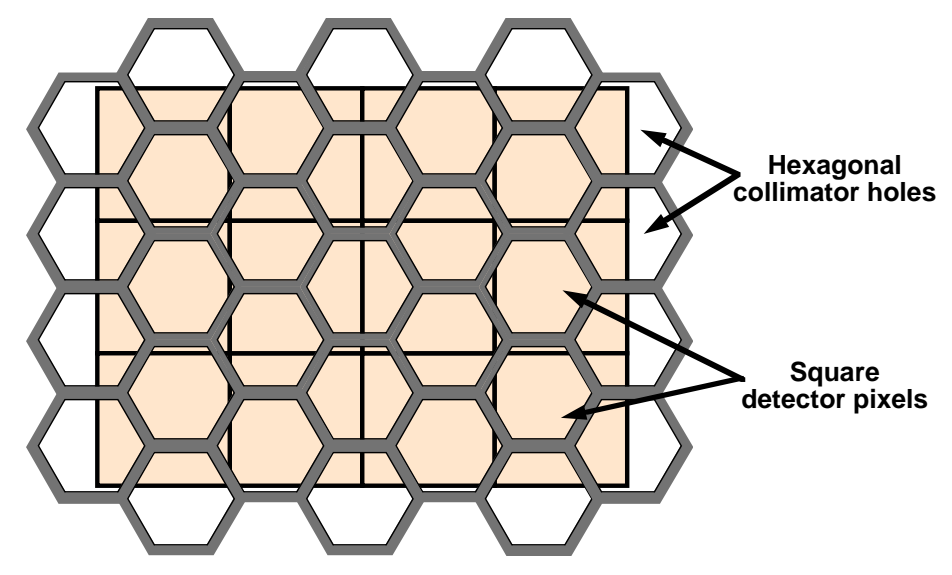

Figure 5.5: Geometric mismatch between hexagonal collimator holes and square detector pixels. Minor artifacts such as increased system spatial resolution and a location-dependent point spread response can result because some collimator holes overlap with multiple pixels. In this example the collimator hole diameter is about half the pixel size, which is the approximate threshold at which this mismatch begins to affect image quality. 
or $10.5 \mathrm{~mm}$ in length, yielding sensitivities of 4000,8000 , or 16,000 counts $/ \mathrm{mCi} / \mathrm{sec}$, respectively. For the square hole collimators the channels are matched 1-to-1 to the $2 \times 2 \mathrm{~mm}^{3}$ pixels and are 43,31 , or $22 \mathrm{~mm}$ in length to provide the same sensitivities, respectively, as the hexagonal hole collimators. For all configurations the septal thickness is $0.2 \mathrm{~mm}$, and the impact this has on collimator sensitivity is fully realized in the code (with the exception of septal penetration, which is not modeled). In the case of the high sensitivity collimators this $0.2 \mathrm{~mm}$ septal thickness would, in reality, result in a rather high septal penetration of about $6.6 \%$. This much penetration may be acceptable in a high sensitivity collimator, but since $5 \%$ penetration is generally the cutoff in collimator design there is some cause for concern. Collimator manufacturers such as Nuclear Fields, Inc., Des Plaines, IL, however, do regularly manufacture ultra high sensitivity collimators with septal penetrations in this range which they regard as appropriate for certain medical applications. In any case, increasing the septal thickness for the high sensitivity collimator designs to $0.25 \mathrm{~mm}$ decreases the penetration to about $5 \%$ while decreasing the collimator sensitivity by only about $10 \%$. This adjustment thus has a relatively minor effect on performance, but it should be considered when designing a high (or ultra high) sensitivity collimator for actual fabrication.

Little difference between hexagonal and square holes is apparent, as hexagonal holes produce tumor fwhm and $\mathrm{S} / \mathrm{N}$ values that on average are $0.1 \mathrm{~mm}$ greater and $2.3 \%$ smaller, respectively, than those observed when using square holes. Collimator sensitivity has only a small impact on observed tumor fwhm-increasing the sensitivity from 4000 to 8000 counts $/ \mathrm{mCi} / \mathrm{sec}$ increases the tumor fwhm an average of $0.4 \mathrm{~mm}$, and increasing the sensitivity again to 16,000 counts $/ \mathrm{mCi} / \mathrm{sec}$ increases the average fwhm only an additional $0.5 \mathrm{~mm}$. Observed tumor $\mathrm{S} / \mathrm{N}$, however, is highly dependent on collimator sensitivity, as the increase from 4000 to 8000 counts $/ \mathrm{mCi} / \mathrm{sec}$ increases the $\mathrm{S} / \mathrm{N}$ an average of $39 \%$, while the increase from 8000 to 16,000 counts $/ \mathrm{mCi} / \mathrm{sec}$ on average increases it an additional $36 \%$. $\mathrm{S} / \mathrm{N}$ is also strongly influenced by tumor depth: at $55 \mathrm{~mm}$ depth the $\mathrm{S} / \mathrm{N}$ averages only 


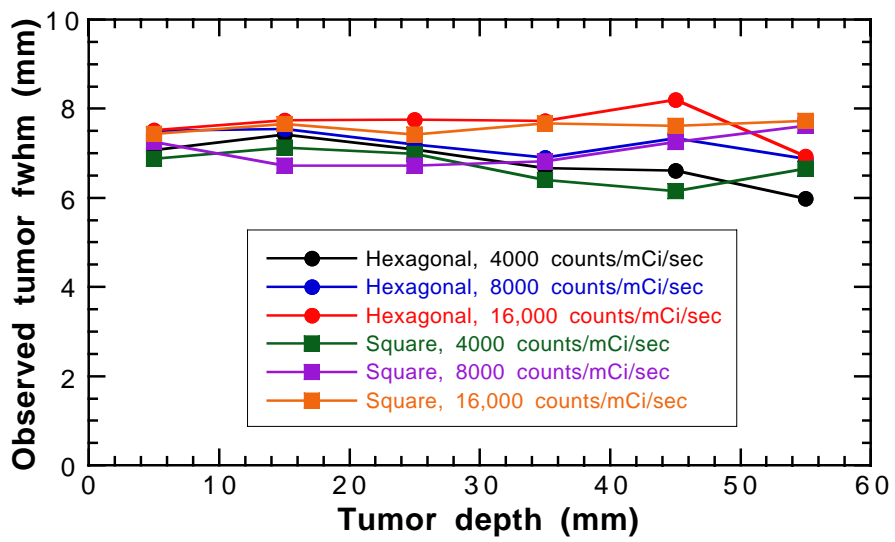

Figure 5.6: Dependence of the observed tumor fwhm on the choice of collimator. The tumor being imaged is $10 \mathrm{~mm}$ in diameter with a $\mathrm{T} / \mathrm{B}$ of 10 , while the pixel size is $2 \times 2 \mathrm{~mm}^{2}$.

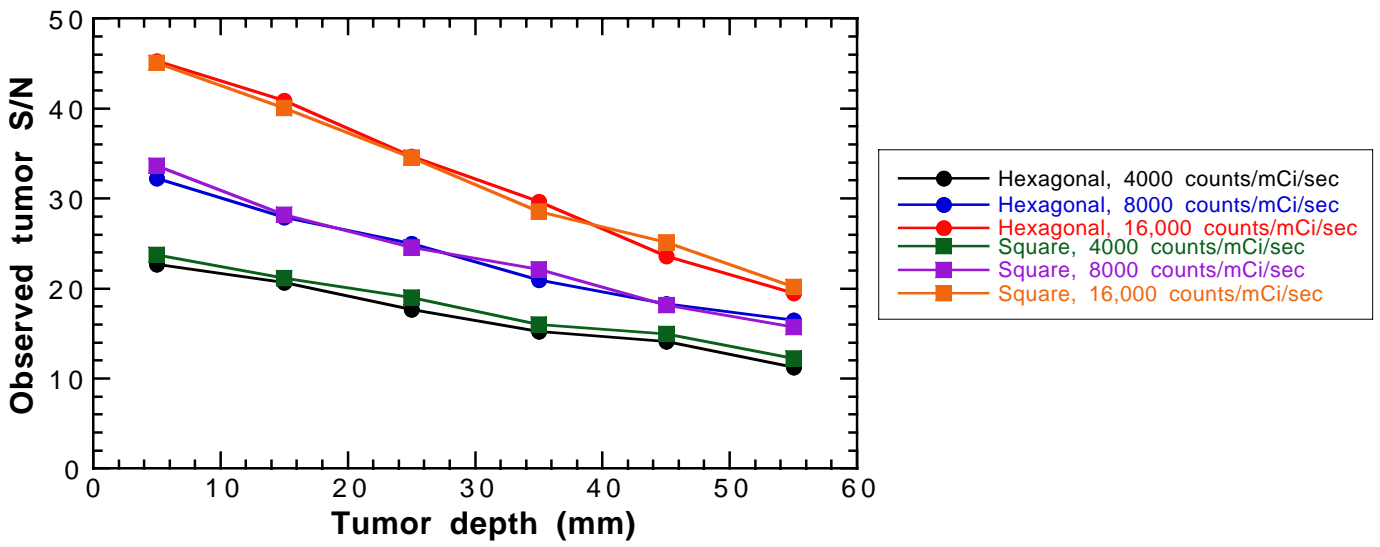

Figure 5.7: Dependence of the observed tumor $\mathrm{S} / \mathrm{N}$ on the choice of collimator. The tumor being imaged is $10 \mathrm{~mm}$ in diameter with a $\mathrm{T} / \mathrm{B}$ of 10 , while the pixel size is $2 \times 2 \mathrm{~mm}^{2}$. 
$48 \%$ of its value at $5 \mathrm{~mm}$ depth. This is primarily due to the attenuation of tumor photons, as $140 \mathrm{keV}$ gammas have an attenuation length of about $67 \mathrm{~mm}$ in water. Trends and conclusions for 7.5 and $15 \mathrm{~mm}$ diameter tumors are identical.

The tumor fwhm in Figure 5.6 does not worsen significantly with increased depth because the tumors are relatively close to the collimator and are large compared to the collimator spatial resolutions at those imaging distances. Thus it is the tumor size, not the collimator spatial resolution, that is the dominant factor in determining tumor fwhm over the range of distances from 5 to $55 \mathrm{~mm}$. At greater distances not germane to the problem of breast imaging, collimator spatial resolution (including its distance dependent behavior) becomes dominant. An example of this phenomenon is depicted in Figure 5.8 - in this case the domain in which the tumor fwhm is dominated by collimator spatial resolution begins at an imaging distance of about $70 \mathrm{~mm}$. The end result is that scintimammography can enjoy the improved statistics of higher sensitivity collimators without significantly sacrificing observed tumor fwhm.

\subsubsection{Pixel Size}

Smaller pixels improve intrinsic spatial resolution and may therefore potentially improve system spatial resolution. Additionally, smaller pixel size means that the photodiodes used to read out individual scintillator crystals are also smaller, hence they demonstrate less per pixel capacitance and dark current, decreasing electronic noise as per Equations 4.12 and 4.13 and thereby improving detector energy resolution. However, decreasing pixel size also increases the density of the electronics required to read out the detector array, which can pose some serious challenges to compact design.

Figures 5.9 and 5.10 display simulation results for the observed tumor fwhm and $\mathrm{S} / \mathrm{N}$, respectively, for different pixel and tumor sizes. Decreasing pixel size slightly decreases the tumor fwhm (more so for tumors $\leq 10 \mathrm{~mm}$ in diameter): de- 


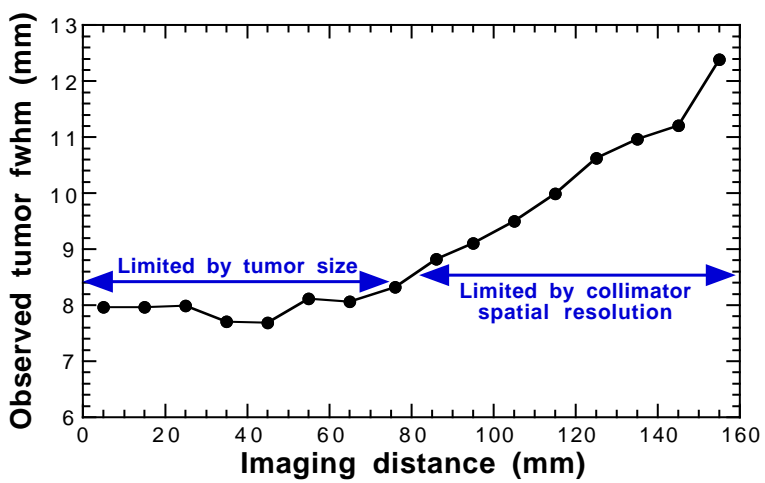

Figure 5.8: Tumor size, not collimator spatial resolution, dominates the observed tumor fwhm at short imaging distances. Shown are simulation results for a $10 \mathrm{~mm}$ tumor imaged in 10 minute simulated scans with $3 \times 3 \mathrm{~mm}^{2}$ pixels and an 8000 counts $/ \mathrm{mCi} / \mathrm{sec}$ hexagonal hole collimator. Two domains are clearly evident: 0-70 $\mathrm{mm}$ where the tumor fwhm is determined by tumor size and is independent of imaging distance, and $\geq 70 \mathrm{~mm}$ where tumor fwhm increases linearly with imaging distance and is clearly influenced by collimator spatial resolution.

creasing pixel size from $4 \times 4 \mathrm{~mm}^{2}$ to $3 \times 3 \mathrm{~mm}^{2}$ results in an average fwhm decrease of $0.7 \mathrm{~mm}$, while $2 \times 2 \mathrm{~mm}^{2}$ pixels yield a further average decrease of $0.2 \mathrm{~mm}$. Observed $\mathrm{S} / \mathrm{N}$ demonstrates little dependence on pixel size - for $2 \times 2 \mathrm{~mm}^{2}$ pixels the $\mathrm{S} / \mathrm{N}$ averages $1.2 \%$ higher than for $3 \times 3 \mathrm{~mm}^{2}$ pixels, which in turn averages $4.7 \%$ larger than the $\mathrm{S} / \mathrm{N}$ for $4 \times 4 \mathrm{~mm}^{2}$ pixels. Finally, the observed $\mathrm{S} / \mathrm{N}$, as expected, depends strongly on tumor diameter: the $\mathrm{S} / \mathrm{N}$ for $15 \mathrm{~mm}$ tumors is on average $120 \%$ larger than for $10 \mathrm{~mm}$ tumors, which in turn averages $85 \%$ larger than the $\mathrm{S} / \mathrm{N}$ for $7.5 \mathrm{~mm}$ tumors.

\subsubsection{Tumor-to-Background Tissue Uptake Ratio}

While a T/B of 10 has been assumed for most of the simulated image acquisitions (Figures 5.3, 5.6, 5.7, 5.9, 5.10, 5.12, and 5.13), estimates of the actual uptake ratio for breast tumors in vivo vary substantially but are usually less than 


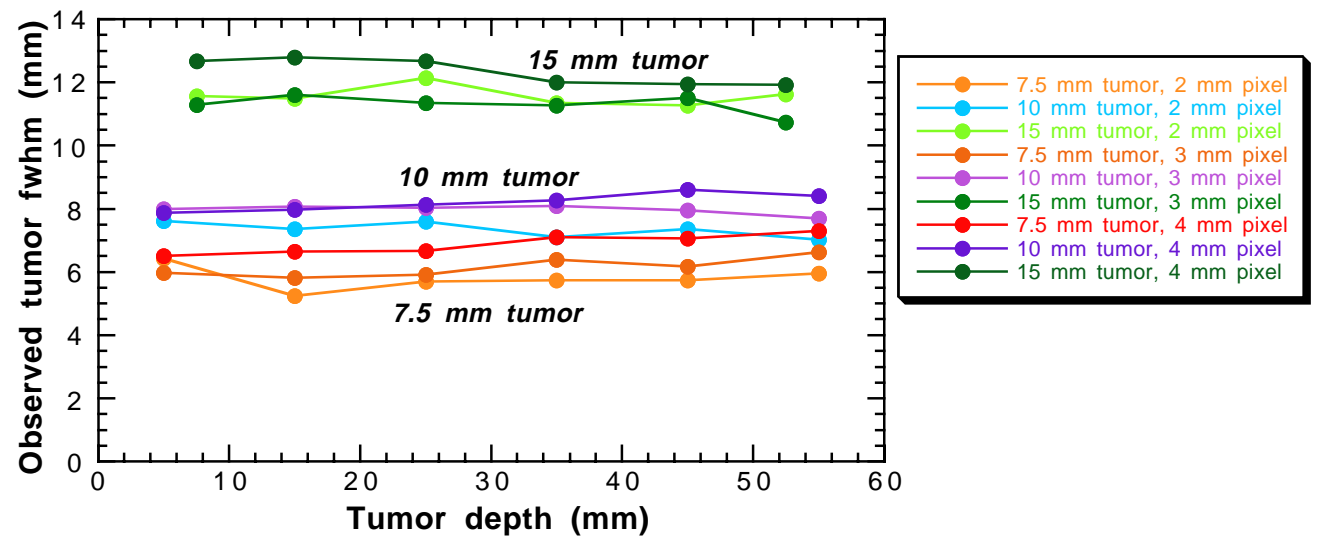

Figure 5.9: Dependence of the observed tumor fwhm on the tumor diameter and detector pixel size. The collimator employed has hexagonal holes and a sensitivity of 8000 counts $/ \mathrm{mCi} / \mathrm{sec}$, while a $\mathrm{T} / \mathrm{B}$ of 10 is assumed.

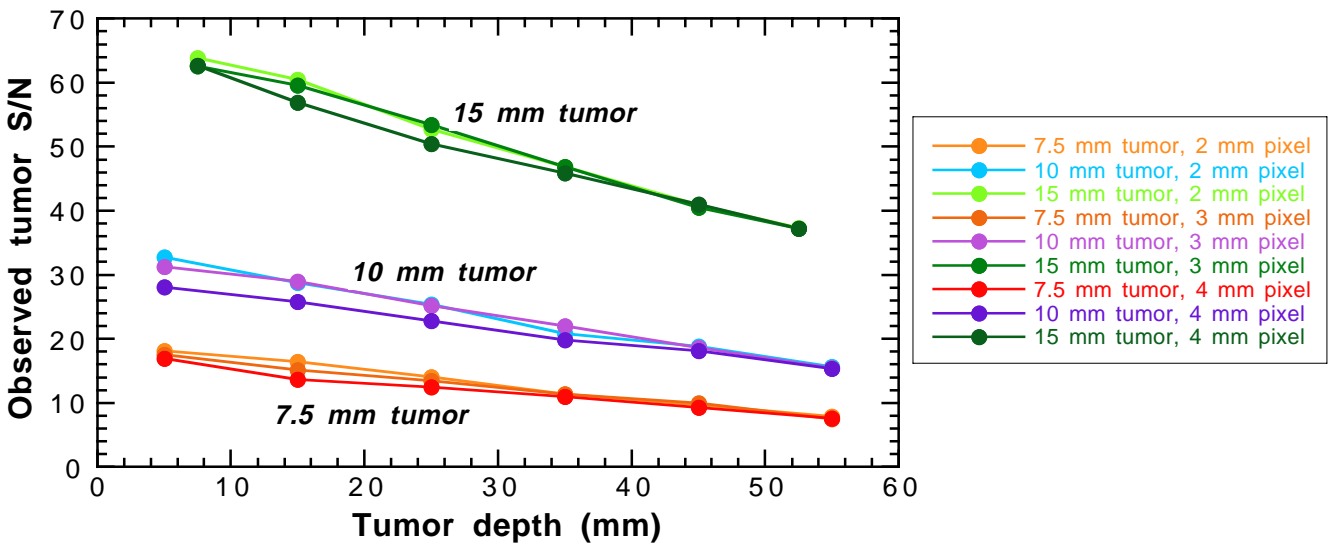

Figure 5.10: Dependence of the observed tumor $\mathrm{S} / \mathrm{N}$ on the tumor diameter and detector pixel size. The collimator employed has hexagonal holes and a sensitivity of 8000 counts $/ \mathrm{mCi} / \mathrm{sec}$, while a $\mathrm{T} / \mathrm{B}$ of 10 is assumed. 
10. There simply does not appear to be a general consensus on the exact T/B value that should be expected in a ${ }^{99 m}$ Tc-Sestamibi scintimammography study, but a range of values from a minimum near 2 to a maximum near 10 are likely to be seen $[48,49,50]$. The optimistic (but not unrealistic) default $\mathrm{T} / \mathrm{B}$ of 10 was used in order to lessen the statistical noise due to count limitations in the detection of tumor events. Simulations with lower T/B values confirm that this choice does not significantly alter the underlying trends in how tumor fwhm and $\mathrm{S} / \mathrm{N}$ are affected by camera design, which is the focus of this study. The higher $\mathrm{T} / \mathrm{B}$, however, makes such trends easier to observe and compare.

Since the number of detected tumor events scales linearly with $\mathrm{T} / \mathrm{B}$, it remains instructive to consider the impact that the range of $\mathrm{T} / \mathrm{B}$ values likely to be seen in actual scans will have on the acquired images. Simulation results showing the dependence of observed tumor $\mathrm{S} / \mathrm{N}$ on the uptake ratio, $\mathrm{T} / \mathrm{B}$, are given in Figure 5.11 for a range of tumor sizes. The observed $\mathrm{S} / \mathrm{N}$ for tumors with a $\mathrm{T} / \mathrm{B}$ of 10 is on average $75 \%$ larger than for tumors with a $\mathrm{T} / \mathrm{B}$ of 5 , which in turn demonstrate an observed S/N that averages $120 \%$ larger than for tumors with a T/B of 2. Also of note is that the $7.5 \mathrm{~mm}$ tumor with a $\mathrm{T} / \mathrm{B}$ of 5 and both the 7.5 and the $10 \mathrm{~mm}$ tumors with a $\mathrm{T} / \mathrm{B}$ of 2 exhibit an observed $\mathrm{S} / \mathrm{N}$ of less than 10 even at a depth of only $5 \mathrm{~mm}$, suggesting that smaller tumors ( $\leq 10 \mathrm{~mm}$ in diameter) with a low T/B $(\leq 5)$ will be challenging to detect. Again, an ROC study would be necessary to quantify true tumor detectability, but it is generally accepted that in observer problems similar to this one where the Rose model is appropriate, a $\mathrm{S} / \mathrm{N}$ of 5 is a reasonable estimate for the threshold at which the feature can be detected [133].

\subsubsection{Detector Energy Resolution}

Variations in detector energy resolution within the range of $5-15 \%$ fwhm have little impact on either observed tumor fwhm or $\mathrm{S} / \mathrm{N}$ for the simulated imaging situation, as shown in Figures 5.12 and 5.13. Compared to an energy resolution of 


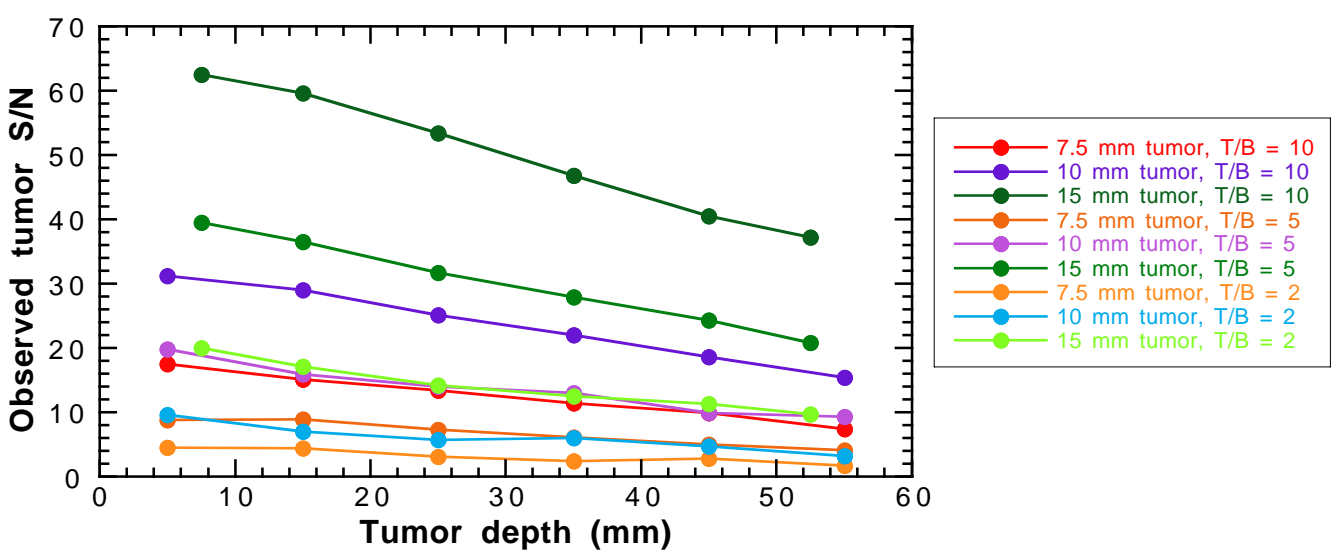

Figure 5.11: Dependence of observed tumor S/N on the tumor-to-background tissue uptake ratio, T/B. For all curves the collimator has hexagonal holes and a sensitivity of 8000 counts $/ \mathrm{mCi} / \mathrm{sec}$, while the pixel size is $3 \times 3 \mathrm{~mm}^{2}$.

$15 \%$ fwhm (with a 119-161 keV window), a 5\% fwhm energy resolution (with a 133$147 \mathrm{keV}$ window) provides an average observed tumor fwhm only $0.1 \mathrm{~mm}$ smaller and an average $\mathrm{S} / \mathrm{N}$ only $5.2 \%$ larger. This insensitivity to energy resolution results because most background photons that reach the detector crystals are unscattered (i.e., $140 \mathrm{keV}$ ) gammas from the breast, and therefore scattered photons as a whole have little impact on image contrast. Photons originating in the torso and heart average only 117 and $114 \mathrm{keV}$, respectively, and can be rejected even with modest energy resolution. As a result, for 5-15\% fwhm energy resolution scattered gammas account for only about $20 \%$ of the image-forming photons.

\subsection{Summary and Analysis}

In terms of observed tumor fwhm and $\mathrm{S} / \mathrm{N}$, hexagonal hole $(1.0 \mathrm{~mm}$ diameter) collimators perform nearly as well as square hole collimators matched 1-to-1 to the detector pixels. Further, hexagonal hole collimators have a more symmetric septal penetration pattern and are more readily available from industry. The geo- 


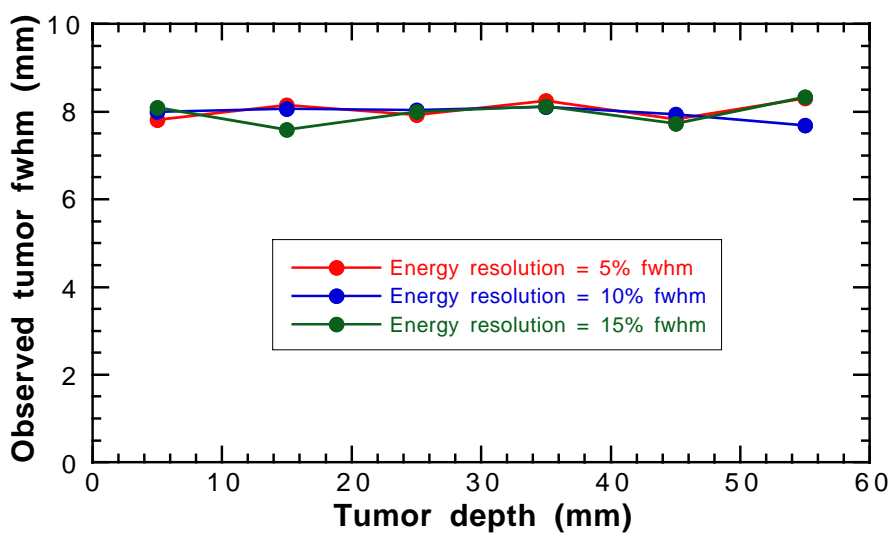

Figure 5.12: Dependence of observed tumor fwhm on detector energy resolution. The tumor has a $10 \mathrm{~mm}$ diameter and a $\mathrm{T} / \mathrm{B}$ of 10, the collimator has hexagonal holes and a 8000 counts $/ \mathrm{mCi} / \mathrm{sec}$ sensitivity, and the pixel size is $3 \times 3 \mathrm{~mm}^{2}$.

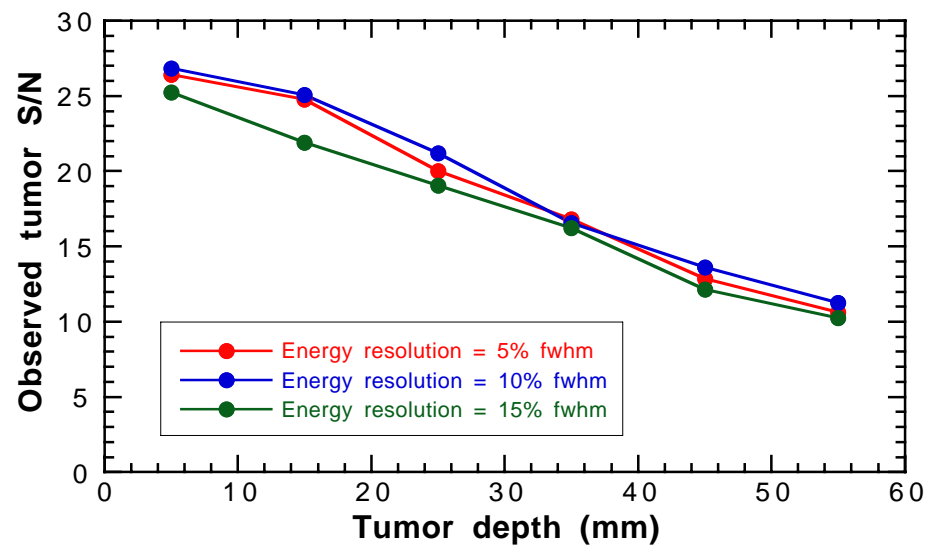

Figure 5.13: Dependence of observed tumor $\mathrm{S} / \mathrm{N}$ on detector energy resolution. The tumor has a $10 \mathrm{~mm}$ diameter and a $\mathrm{T} / \mathrm{B}$ of 10 , the collimator has hexagonal holes and a 8000 counts $/ \mathrm{mCi} / \mathrm{sec}$ sensitivity, and the pixel size is $3 \times 3 \mathrm{~mm}^{2}$. 
metric mismatch between hexagonal collimator holes and square detector pixels has the potential to slightly increase pixel-to-pixel variations in sensitivity, but pixel-topixel sensitivity variations need to be corrected through calibration regardless of the choice of collimator.

Since the tumors expected to have enough activity to be detected during a 10 minute scan are relatively large $(\geq 7.5 \mathrm{~mm})$, tumor size tends to dominate observed tumor fwhm more than either pixel size or collimator spatial resolution. As a result, the benefits of small pixels and high resolution collimators are marginal. A collimator with a high sensitivity of 16,000 counts $/ \mathrm{mCi} / \mathrm{sec}$ yields significantly better tumor $\mathrm{S} / \mathrm{N}$ values than does an all purpose collimator with a sensitivity of 8000 counts $/ \mathrm{mCi} / \mathrm{sec}$, which in turn produces higher $\mathrm{S} / \mathrm{N}$ values than a high resolution collimator with 4000 counts/mCi/sec sensitivity. Further, higher collimator sensitivity only slightly increases observed tumor fwhm. Thus a high sensitivity hexagonal hole collimator is a wise design choice for this application.

The improved intrinsic spatial resolution offered by smaller pixels does slightly decrease tumor fwhm, especially for tumors $\leq 10 \mathrm{~mm}$ in diameter. Further, slight increases in observed tumor $\mathrm{S} / \mathrm{N}$ are realized with smaller pixels since they are better able to conform to non-square tumor shapes. However, for both metrics the gains are small and show diminishing returns - the improvement realized by going from $3 \times 3$ to $2 \times 2 \mathrm{~mm}^{2}$ pixels is significantly less than realized by going from $4 \times 4$ to $3 \times 3 \mathrm{~mm}^{2}$ pixels. Thus, decreasing photodiode capacitance and dark current appear to be the only compelling reasons to decrease pixel size much below $3 \times 3 \mathrm{~mm}^{2}$, and that must be weighed against the difficulty and expense of increasing the density of the readout electronics.

Finally, variations in detector energy resolution over the range of $5-15 \%$ fwhm have very little impact on either the observed tumor fwhm or $\mathrm{S} / \mathrm{N}$. This is in part due to the favorable imaging geometry assumed (Figure 5.1) wherein the camera does not look directly at either the heart or torso. Further, in pixellated cameras gamma ray location determination is discretized and is thus independent 
of energy resolution (unlike in cameras using Anger logic). In contrast with these results, reference [134] suggests that energy resolution is indeed important for scintimammography because of the prevalence of Compton-scattered events reaching the detector. However, the phantom geometry in that work was different, the scintimammography camera was not pixellated, and the range of energy resolutions examined was considerably larger at $10-30 \%$ fwhm. Since $140 \mathrm{keV}$ energy resolution of $8-11 \%$ fwhm has already been demonstrated with discrete scintillator/photodiode camera technology [93, 94] and 4\% fwhm has been achieved with CdZnTe detector arrays [105], the range of energy resolutions examined in this work would seem to be reasonable. 


\section{Chapter 6}

\section{Custom Integrated Circuit Readout}

It looked like a useful idea and one that was worth pursuing.

— Jack Kilby, co-inventor of the integrated circuit

\subsection{Basic PETRIC Design}

Motivated by the goal of compact design and the need to process potentially 1000 s of channels of electric signals, my colleagues and I have designed an ASIC dedicated to reading out scintillator/silicon PIN photodiode detector channels [135]. The ASIC is one of the most innovative aspects of the camera development described in this thesis because while several other research groups have presented similar prototype $\mathrm{CsI}(\mathrm{Tl}) /$ photodiode imaging technology [92, 94, 96], none have described an electronic readout system that could feasibly be employed in a compact camera with a FOV large enough to image the breast. Compactness is critical, of course, to achieving the breast-imaging advantages discussed in Section 3.2.1. Undoubtedly part of the reason no other readout ASICs for compact gamma cameras have yet to be presented is the huge development time, effort, and expertise that is required. 
Our ASIC, for instance, has involved multiple design iterations and a great deal of prototype IC testing and debugging over a period of seven years.

The ASIC is known as the PETRIC-Positron Emission Tomography Readout Integrated Circuit-because the IC is as applicable to LSO/photodiode PET technology as it is to $\mathrm{CsI}(\mathrm{Tl}) /$ photodiode single photon cameras. The design implements a 64-channel front end comprised of a low-noise charge-sensitive preamplifier and an RC-CR shaper amplifier for each pixel, much like in Figure 4.1. In addition to the compactness afforded by realizing these circuits in an IC rather with discrete electronics, the IC achieves even greater compactness by following the 64 front end channels with a 64-input WTA multiplexer that selects the channel with the largest input signal and generates a 6 -bit digital address indicating the winner. This reduces the required output lines from 64 analog signals to 1 analog line plus 6 digital lines, making routing issues substantially simpler. The single analog output is simply the front end waveform with the greatest voltage buffered onto the line, and therefore it carries all the information of the shaper output (Figure 4.3). The IC is fabricated in $0.5 \mu \mathrm{m} 3.3 \mathrm{~V}$ standard CMOS technology and is approximately $4.5 \times 4.8 \mathrm{~mm}^{2}$ in size. It represents the logical next stage of development following the IC readout scheme described in Chapter 4.

In addition to the front end and the WTA, the PETRIC also includes a digital control section that has been synthesized from a state machine diagram using standard cells. The digital protocol for controlling the IC is based on the Xicor $\mathrm{I}^{2} \mathrm{C}$ (Inter-IC bus) communication system and therefore includes an SDA serial data line for sending the chip commands and an SCL serial clock line for latching in those commands. Proper setting of the flip-flops in the digital control section allows manipulation of:

- Multiplexer mode: the WTA section can be set to select the channel with the greatest signal or it can be set to continuously select one specific channel.

- Masking: any given channel in the WTA can be turned off. 
- Calibration: the compensation current required to sink the detector input current and thereby prevent preamplifier saturation can be controlled on a channelby-channel basis to a precision of 4 bits.

- Gain: the gain of the front end can be controlled to a precision of 6 bits.

- Read mode: the state machine's registers can be read out to confirm the IC's status.

In addition to the $\mathrm{I}^{2} \mathrm{C}$ control capabilities, external current supplies set the rise time and fall time of the shaping amplifiers, allowing external control of these two parameters.

Another critical feature of the PETRIC is the inclusion of a $65^{\text {th }}$ "dummy" channel which serves to prevent the 6 WTA address bits from switching in response to noise superimposed upon the DC baseline input signals. The threshold set by this dummy channel is about $50 \mathrm{mV}$ above the quiescent voltage of the other channels (though this threshold can be adjusted externally), and therefore the dummy channel is always selected except when one of the true channels experiences a significant input signal. This prevents the address bits from chattering needlessly and potentially coupling noise back into the charge-sensitive amplifiers. Finally, the PETRIC includes track-and-hold capabilities for the analog winner signal, but this is not utilized in the single photon camera architecture described in this thesis.

Figure 6.1 and Table 6.1 summarize the PETRIC design. A CMOS $0.5 \mu \mathrm{m} 3.3 \mathrm{~V}$ fabrication technology was chosen largely because of its availability - the CMOS $1.2 \mu \mathrm{m} 5.0 \mathrm{~V}$ technology that had been successfully used with the prototype ASICs in Chapter 4 had become obsolete and was being phased out. The input load capabilities had to be able to handle a range of photodiodes, and $600 \mathrm{pA}$ current and $5 \mathrm{pF}$ capacitance ensured that a wide range of such detectors could be used. A maximum gain of $600 \mathrm{mV} / \mathrm{fC}$ was implemented because, as was shown by the success of the 16-channel front end prototype ASIC, it provides a large output signal reasonably immune to noise $\left(481 \mathrm{mV}\right.$ for a $5000 \mathrm{e}^{-}$input while maintaining linearity of 
output voltage versus input charge. Larger gain becomes increasingly dangerous because of the risk of positive feedback loops in the PETRIC, leading to catastrophic oscillations. The target noise value of $150 \mathrm{e}^{-}$rms at $2 \mu$ s peaking time (optimal for minimizing electronic noise) with a photodiode load is important to maintaining good energy resolution that is not dominated by electronic noise. The very early 16-channel front end ASIC explored in Chapter 4 demonstrated this noise level and a corresponding $180 \mathrm{e}^{-}$rms at $8 \mu$ s peaking time, yielding an electronic noise of only $5.9 \%$ fwhm for the $6600 \mathrm{e}^{-}$signals generated by ${ }^{99 m} \mathrm{Tc}$ interactions (Section 4.3.2).

The remainder of this chapter describes the implementation and performance of both the PETRIC and the prototype ICs upon which it is based. While this ASIC design effort has involved numerous individuals, my specific contributions have include developing IC specifications, analyzing and debugging multiple prototype front end ICs, applying the knowledge gained from my characterization of the readout scheme described in Chapter 4 to the final PETRIC design, and testing and debugging the final PETRICs. Some of these efforts are highlighted in Section 6.4.1, which lists the design changes that were implemented based on measurements with the prototype ICs. Chapter 7 describes the performance of a complete single photon imaging system and addresses the role the PETRIC plays in this architecture, in-

cluding my design of the readout/control hierarchy and the computer interface used to manage multiple PETRICs in a multi-module imaging array.

\subsection{Analog Front End}

The use of CMOS amplifiers for nuclear medicine front end electronics was evaluated in [119] and tested in the prototype 12-pixel module described in Chapter 4. In both cases the IC under consideration was a preliminary version of these electronics (version three in both cases). My final evaluation of the front end design (version five) prior to implementation of the 64-channel PETRIC chip is described herein. 


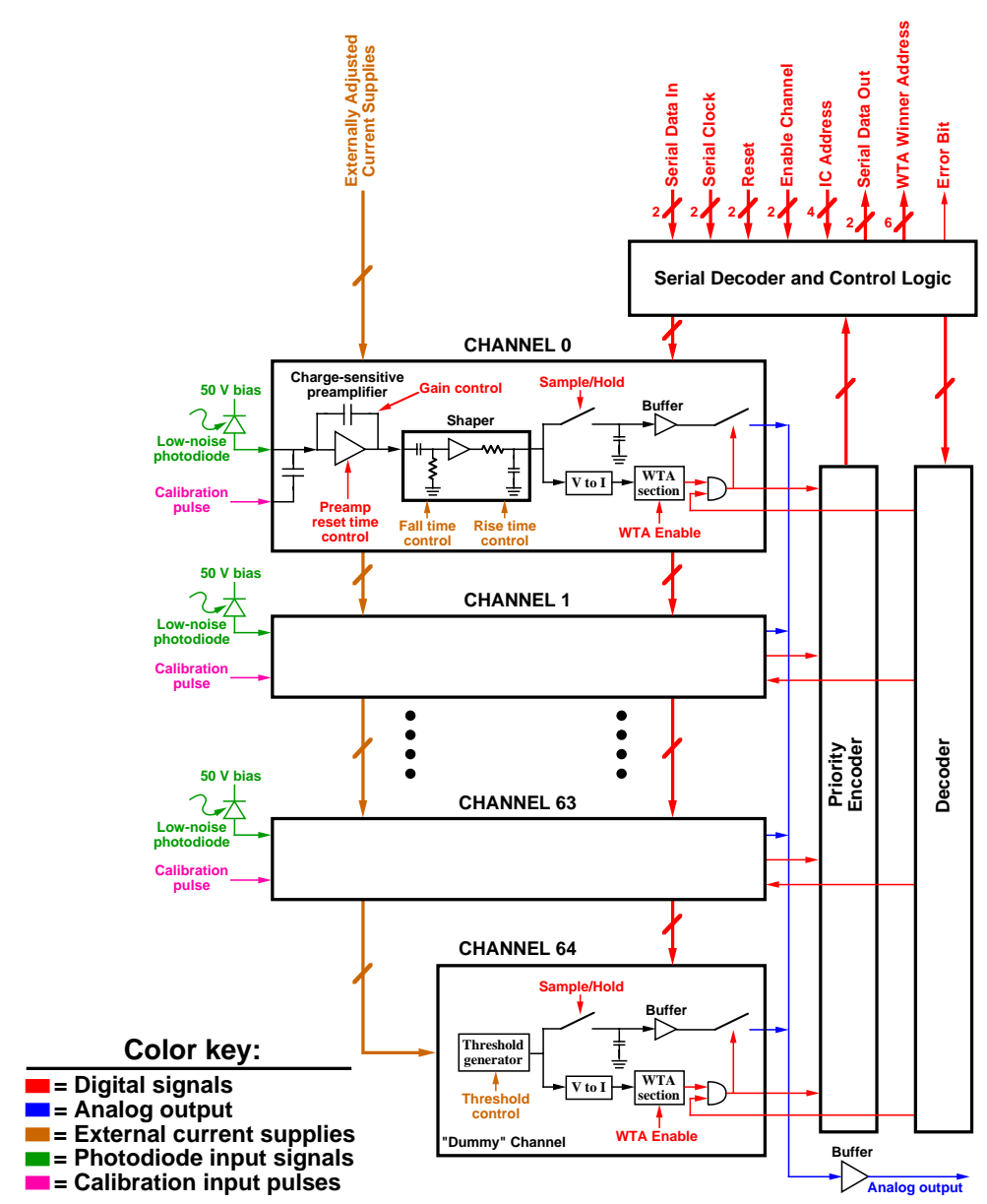

Figure 6.1: Block diagram of the PETRIC. Input signals consisting of charge generated in the photodiodes are preamplified and filtered to optimize the signal-to-noise ratio. The digital signal generated by the WTA section within each channel indicates whether or not that particular channel is the winner. Using these bits, the WTA circuitry continuously selects the channel with the largest signal and connects it to an output buffer. A $65^{\text {th }}$ "dummy" channel prevents the WTA from unnecessarily switching these digital lines in response to noise. A priority encoder and error logic produce an error bit either when no channel or more than one channel are selected by the WTA. The control logic sets the gain and the reset time constant for the preamplifiers, while the rise and fall times of the shaper amplifiers are set by external current supplies. Each channel includes a calibration capacitor as a means of injecting a known input charge for testing purposes. A sample and hold capacitor can store the analog peak voltage, but this feature is not utilized in this thesis. 


\begin{tabular}{|c|c|}
\hline Technology & CMOS $0.5 \mu \mathrm{m} 3.3 \mathrm{~V}$ \\
\hline Number of transistors & $\sim 50,000$ \\
\hline Number of detector channels & $64(+1$ dummy channel $)$ \\
\hline Input leakage current range & $0-600 \mathrm{pA}$ \\
\hline Input capacitance range & $0-5 \mathrm{pF}$ \\
\hline Gain & $75-600 \mathrm{mV} / \mathrm{fC}$ in 64 steps \\
\hline Peaking time & $0.5-15 \mu \mathrm{s}$ \\
\hline Noise & $150 \mathrm{e}^{-} \mathrm{rms} @ 2 \mu \mathrm{s}$ peaking, $50 \mathrm{pA}$, and $3 \mathrm{pF}$ \\
\hline Power & $5.4 \mathrm{~mW}$ per channel \\
\hline Die area & $4.5 \times 4.8 \mathrm{~mm}^{2}$ \\
\hline
\end{tabular}

Table 6.1: Summary of the primary PETRIC characteristics.

\subsubsection{Charge-Sensitive Preamplifier}

A simplified circuit diagram for a single preamplifier channel is displayed in Figure 6.2. The design utilizes a single stage common-source cascode amplifier with a cascode active load. A p-channel FET is employed as the input transistor, $\mathrm{M}_{1}$, because of its lesser white and $1 / \mathrm{f}$ noise in comparison to $\mathrm{n}$-channel devices. The preamplifier is optimized with regard to thermal and $1 / \mathrm{f}$ noise for a detector capacitance of $3 \mathrm{pF}$. Adjusting the value of $C_{\text {feedback }}$ sets the preamplifier gain as per Equation 4.2. MOS switches controlled by 6 digital bits determine the value of $C_{\text {feedback }}$ by connecting or disconnecting a series of capacitors (each sized twice as large as the previous one) arranged in parallel in the feedback path. The GainBandwidth (GBW) product of the amplifier is given by:

$$
G B W=\frac{g_{m 1}}{C_{c}}
$$

where $g_{m 1}$ is the transconductance of $M_{1}$.

The preamplifier reset system employs a single p-channel FET, $\mathrm{M}_{\text {feedback }}$, as per the design proposed in [136], though in the PETRIC and prototype front end 


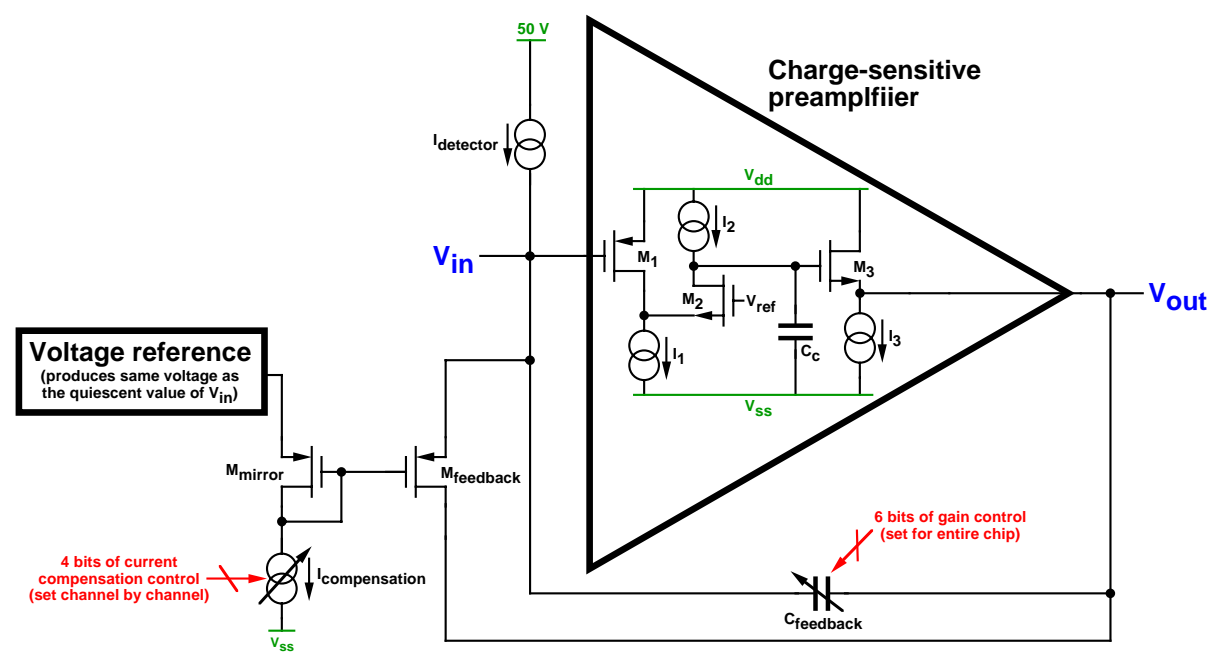

Figure 6.2: Simplified circuit diagram of a single charge-sensitive preamplifier channel. Innovative features include the adjustable gain, the adjustable compensation for the detector current, and the use of a feedback transistor to provide a low-noise means of both compensating for the input current and discharging the feedback capacitor.

a minimum length transistor $(2.4 \mu \mathrm{m} / 0.6 \mu \mathrm{m})$ operated in the saturation region is used to achieve a high source-to-drain resistance (on the order of 10 G $\Omega$ ). This current path serves not only to sink the input leakage current from the photodiode detector (up to $600 \mathrm{pA}$ ) and thereby prevent preamplifier saturation, it also provides a discharge path for the charge injected onto $C_{\text {feedback }}$.

To cope with the large variation in leakage current than can exist between different pixels, the operating point of $M_{\text {feedback }}$ is controlled via an arrangement that approximates a current mirror. Because the $V_{G S}$ values for $M_{\text {mirror }}$ and $M_{\text {feedback }}$ are nearly identical, the current running through $\mathrm{M}_{\text {feedback }}$ can be controlled by adjusting the magnitude of $I_{\text {compensation. }}$ Much like with the feedback capacitance, this is achieved using FET switches controlled by 4 digital bits which connect and disconnect a series of current sources arranged in parallel. Generally it is best for $I_{\text {compensation }}$ to be set such that it is slightly larger than $I_{\text {detector }}$ and barely prevents 
preamplifier saturation. This is because the effective resistance of a FET transistor depends on its bias current [137]:

$$
R_{\text {equivalent }}=\frac{1}{\sqrt{2 I_{D} \mu C_{o x} \frac{W}{L}}}
$$

where $I_{D}$ is the drain current through the FET device, $\mu$ is the carrier mobility, $C_{o x}$ is the oxide capacitance, $W$ is the transistor width, and $L$ is the transistor length. Thus the minimum current, $I_{D}$, which prevents saturation will result in the maximum effective resistance for $\mathrm{M}_{\text {feedback }}$ and, as described in Section 4.2.3, will provide the lowest possible electronic noise.

The value of $R_{\text {equivalent }}$ for $M_{\text {feedback }}$ also has an obvious impact on the reset time of the preamplifier. Since $C_{\text {feedback }}$ is discharged through that equivalent resistance, the $\tau$ for the preamp reset is approximately $R_{\text {equivalent }} C_{\text {feedback. This is }}$ yet further cause for the value of $R_{\text {equivalent }}$ to be made as large as possible without sending the preamp into saturation, for if the reset time becomes too short the shaper output will become distorted and experience a loss of signal.

\subsubsection{Shaper Amplifier}

The pulse shaper shown in Figure 6.3 is based on the design presented in [138] and consists of a two-stage $\mathrm{G}_{m}-\mathrm{C}$ CR-RC filter. Two external current supplies allow independent control of the rise and fall times so that the signal-to-noise of the output pulse can be maximized. In order to ease the requirements on the large signal linearity of the transconductors in the shaping blocks, the shaping circuitry shown in Figure 6.3 is followed by another amplifier stage that does no further pulse-shaping. The cost of this approach is increased power consumption compared to a one-stage filter design.

The purpose of the shaper stage is to provide a bandpass filter with cutoff frequencies that are externally adjustable. This is achieved by implementing two operational transconductance amplifiers (OTAs), each of which requires an external 


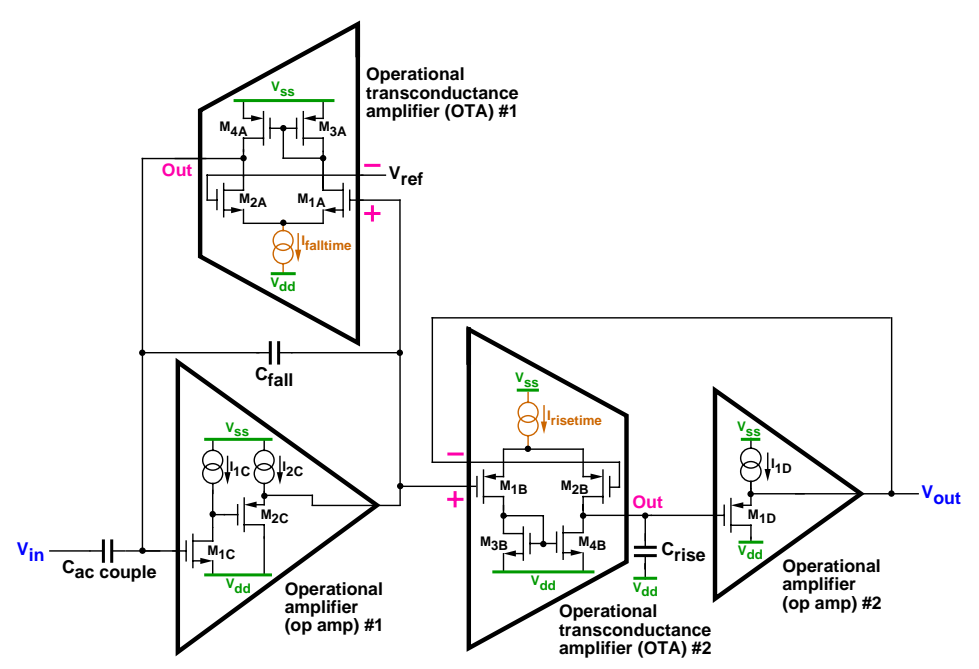

Figure 6.3: Simplified circuit diagram of a single shaper amplifier channel. The circuit performs pulse shaping by implementing a bandpass filter with cutoff frequencies that are set by the two external current supplies, $I_{\text {falltime }}$ and $I_{\text {risetime }}$.

current supply (namely $I_{\text {falltime }}$ or $I_{\text {risetime }}$ ). The OTA input transistors are configured as a current-starved differential pair with an equivalent resistance (i.e., voltage to current conversion) that is controlled by the external current supply as per the relationship in Equation 6.2. This implementation is used to realize two adjustable low-pass filter stages: (1) OTA \#1 and $C_{\text {fall }}$ form a parallel RC configuration in the feedback loop of op amp \#1, and (2) OTA \#2 and $C_{\text {rise }}$ form a series RC network whose output is buffered by op amp \#2. In addition, $C_{a c}$ couple provides high-pass filtering and completely kills DC components.

Replacing the OTAs with their equivalent resistances leads to the following transfer function:

$$
\frac{V_{\text {out }}}{V_{\text {in }}}=-\frac{j \omega R_{O T A \# 1} C_{\text {ac couple }}}{\left(1+j \omega R_{O T A \# 1} C_{\text {fall }}\right)\left(1+j \omega R_{O T A \# 2} C_{\text {rise }}\right)}
$$

Thus this circuit implements a single zero-frequency zero and two poles whose frequencies are set by $R_{O T A \# 1} C_{\text {fall }}$ and $R_{O T A \# 2} C_{\text {rise }}$, respectively. The first pole sets 
the lower cutoff frequency for the bandpass filter-at frequencies lower than this cutoff the zero provides a gain increase of $20 \mathrm{~dB} /$ decade, while at frequencies above the cutoff the zero and the pole cancel each other out and leave a flat passband. The second pole sets the upper cutoff frequency, and at frequencies greater than that cutoff the pole pulls the output amplitude down at $-20 \mathrm{~dB} /$ decade.

The overall effect is that output pulse fall time is determined by the differentiating - or high-pass filtering - component of the system:

$$
\tau_{\text {fall }} \propto R_{O T A \# 1} C_{\text {fall }}
$$

while the rise time is set by the integrating - or low-pass filtering-portion of the circuit:

$$
\tau_{\text {rise }} \propto R_{O T A \# 2} C_{\text {rise }}
$$

This then gives the user control of the rise and fall of the output pulse via $I_{\text {risetime }}$ and $I_{\text {falltime }}$, as desired.

\subsubsection{Prototype Performance}

I confirmed that the 16-channel front end prototype IC meets the desired specifications in most ways. Using both hand-adjusted potentiometers and a series of computer-controlled DACs, I supplied a range of voltages and currents to a circuit board with a mounted, wirebonded front end ASIC. In addition to this, I used a signal generator to supply a square wave to internal injection capacitors (fabricated within the front end ASIC), allowing me to repeatedly inject a known charge (calibrated using $5.9 \mathrm{keV}$ gammas from ${ }^{55} \mathrm{Fe}$ interacting directly in Si photodiodes) into the preamps. I observed various signals using a digital oscilloscope and collected histograms of the shaped output pulses using a peak-detecting ADC. The performance characteristics I verified include:

- Control of preamp reset time, preamp gain, and shaper rise and fall times operated as expected. I was able to achieve preamp reset times ranging from less 
than $1 \mu \mathrm{s}$ to more than $50 \mu \mathrm{s}$ (either with or without a photodiode load), which met our target specification of 20-50 $\mu$ with a custom Si PIN photodiode load. The preamplifiers slightly exceeded the desired maximum gain of $600 \mathrm{mV} / \mathrm{fC}$. The shaper rise time, with the fall time set as long as possible, was successfully varied from 0.4 to $15 \mu \mathrm{s}$. Likewise, the shaper fall time, with the rise time set as short as possible, was successfully varied from 1.5 to $40 \mu \mathrm{s}$.

- Proper pulse shaping was realized. I was able to simultaneously adjust the shaper rise and fall times to achieve shaped output pulses with a rise time to fall time ratio of about 1:3 over a range of peaking times from 0.5 to $14 \mu \mathrm{s}$. Since the IC is meant to operate near $8 \mu \mathrm{s}$ for this thesis project and near $1 \mu \mathrm{s}$ for PET applications, this definitely meets expectations. The one exception to proper pulse shaping was that, at maximum gain, input signals larger than $3000 \mathrm{e}^{-}$produced a slew rate-limited output pulse.

- Noise performance with and without an input load met expectations. Electronic noise was calculated from the peak fwhm of the pulse height histogram. The most important input load was a custom Si photodiode, and with that load the front end electronic noise was less than $150 \mathrm{e}^{-}$rms at $2 \mu$ s and about $190 \mathrm{e}^{-}$ rms at $8 \mu \mathrm{s}$, compared to target values of 150 and $180 \mathrm{e}^{-} \mathrm{rms}$, respectively. More comprehensive data are presented toward the end of this section.

- Minimal channel-to-channel variations exist in DC output voltages. Across 48 channels on 3 ICs, the standard deviation in the quiescent output voltages is only $6 \mathrm{mV}$. This is substantially lower than the $\sim 25 \mathrm{mV}$ or so required to have a channel selected by the WTA as the winner, so this level of variation is entirely within specs.

Those problems that were uncovered and fixed for the final PETRIC chip are addressed further in Section 6.4.1. 
In addition to the concerns listed above, the prototype front end was designed with four similar but distinctly varied channel types, all of which I tested and compared. These four channel types are permutations of: (1) channels with and without protection diodes on the inputs and (2) channels with two different mechanisms to compensate for the input load current. One of the current compensation mechanisms is that shown in Figure 6.2, while the other is a simple externally-controlled current source connected between the input node and $V_{s s}$. The motivation for implementing these different channel types was to further explore the various possibilities since my observations with previous incarnations of the front end had been limited and therefore inconclusive. Ultimately, however, there was little performance difference between the different versions, so results for all channels are presented together. Final design choices made regarding these different channel types are discussed further in Section 6.4.1.

My evaluation of the prototype front end noise performance is also encouraging. Figure 6.4 shows measured noise curves for all 16 channels in a single prototype IC, demonstrating the effects of peaking time and input load. These measurements were made by loading the front end ASIC with photodiode pixels at $50 \mathrm{~V}$ bias, adjusting the control voltages to realize the desired shaping time, and injecting a known charge into the desired ASIC input channels with a square voltage wave on an injection capacitor. The value of the charge injected was determined by calibrating with the direct interaction of the $5.9 \mathrm{keV}$ gammas from a ${ }^{55} \mathrm{Fe}$ source in the $\mathrm{Si}$ photodiodes (known to produce $1645 \mathrm{e}^{-}$) and assuming linearity. Finally, the peak voltage of the shaped output pulses generated by the front end IC in response to the injected charge were histogrammed, and the fwhm width of the histogram peak used to calculate the electronic noise.

A low-noise photodiode load $(\sim 70 \mathrm{pA}, \sim 3 \mathrm{pF})$ with a leakage current about twice the average for these devices yields good noise performance. At $2 \mu$ s the noise drops below $150 \mathrm{e}^{-}$rms, and even in the $8 \mu$ s range used with $\mathrm{CsI}(\mathrm{Tl})$ detector systems the noise is only about $190 \mathrm{e}^{-}$rms. With a $>6000 \mathrm{e}^{-}$signal this would 


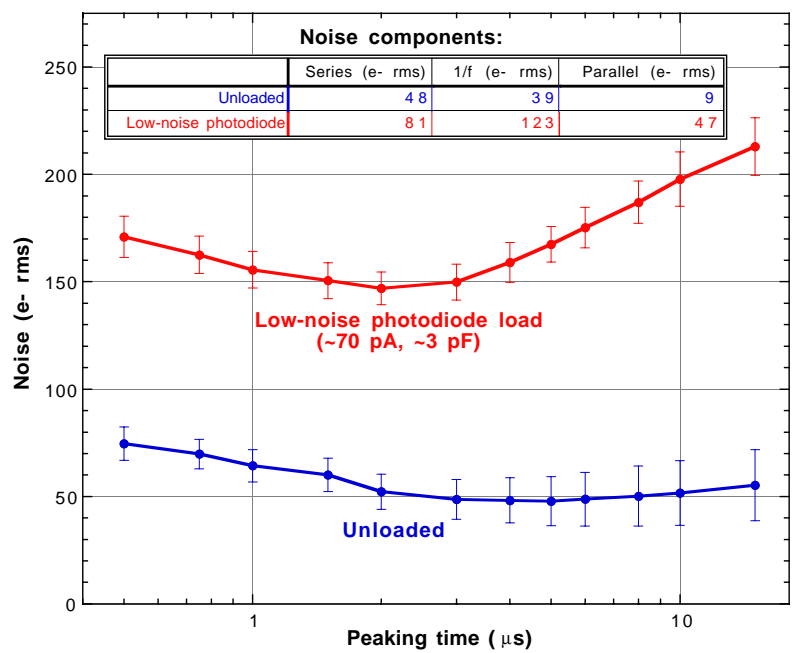

Figure 6.4: Equivalent noise charge versus shaper peaking time for a prototype front end IC with either no input load or a load consisting of a low-noise photodiode $(\sim 70 \mathrm{pA}, \sim 3 \mathrm{pF})$ exhibiting about twice the average leakage current for these devices. Results are averaged across all 16 channels in a single IC. The noise components listed for the two different input loads are taken from a simple curve fit and demonstrate the relative importance of the different types of noise.

contribute an energy resolution error of about $7 \%$ fwhm, significantly below the 9-11\% target range (though of course there are other noise sources in addition to electronic noise). With photodiodes that demonstrate a more typical leakage current of $20-50 \mathrm{pA}$ the results for an $8 \mu$ s peaking time would further improve.

The shapes of the noise curves also meet expectations. Using a simple curve fitting routine based on Equations 4.12 and 4.13:

$$
\text { Noise }=\sqrt{A_{\text {series }}^{2} \frac{1 \mu s}{\text { Peaking time }}+A_{1 / f}^{2}+A_{\text {parallel }}^{2} \frac{\text { Peaking time }}{1 \mu s}},
$$

the contributions of the different types of noise, $A_{\text {type }}$, can easily be determined. In Figure 6.4 I report these values for the two noise curves presented. The photodiode load adds both capacitance and current compared to the unloaded case, therefore both the series and parallel noise components increase. An increase in 1/f noise is 
also expected when adding a load, but the large difference observed between the photodiode and no load cases may in part be due to the simplicity of the curve fit. Data from other input loads also demonstrate consistent trends: (1) increasing the capacitance increases the series noise, (2) increasing the current increases the parallel noise, and (3) 1/f noise progressively increases as the load is increased from no load to capacitors and/or resistors to photodiodes. The IC performs normally with input loads as large as $5 \mathrm{pF}$ and $600 \mathrm{pA}$.

A final consideration for the prototype front end IC is the preamplifier reset time, as this is critical to proper pulse shaping. For any given channel with any reasonable load it is possible to set the compensation current appropriately to achieve good performance, but given channel-to-channel and photodiode-to-photodiode variations my measurements make it clear that it is impossible to set one bias level that adequately serves all channels in a given IC/module. Figure 6.5, for example, shows the preamp reset time versus bias voltage (translated to bias current via a $20 \mathrm{k} \Omega$ resistor) with input load current as a parameter for a single channel. Keeping in mind that $20-50 \mu \mathrm{s}$ is the target reset time (longer reset times may lead to preamplifier saturation, while shorter peaking times can result in ballistic deficit and signal loss), it is clear that in order to keep a $100 \mathrm{pA}$ channel out of saturation, the bias voltage must be set to at least $1.1 \mathrm{~V}$. For any $20 \mathrm{pA}$ pixels in the same module, however, that bias voltage will result in problematically low reset time of just over $10 \mu \mathrm{s}$. Thus in order to achieve proper preamp reset times for all pixels it is necessary to set the bias individually for each channel.

\section{3 "Winner Take All" Mixed Analog/Digital Back End}

The WTA multiplexer was also tested in a prototype 16-channel IC to verify its functionality and aid in final design decisions. Described below is version three of the IC, which is based upon earlier versions described in reference [120]. The ability of the WTA circuitry to reduce the number of analog output signals from a single 


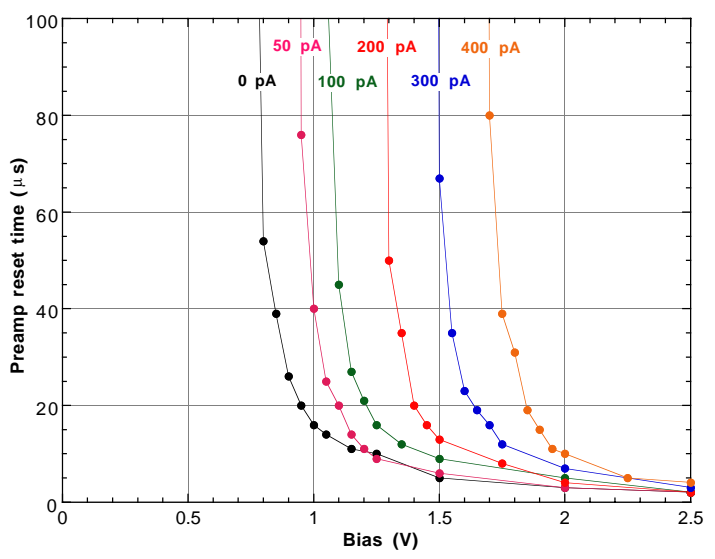

Figure 6.5: Preamplifier reset time versus the current compensation bias voltage (which provides bias current through a $20 \mathrm{k} \Omega$ resistor) with the input load current as a parameter. Load current is supplied through an adjustable resistor that contributes minimal load capacitance. Preferred reset times are in the neighborhood of 20-50 $\mu \mathrm{s}$.

module from 64 (or 16 in the prototype) to one is absolutely essential to the overall system design described in Chapter 7.

\subsubsection{Circuit Design}

A simplified circuit diagram of the heart of the WTA circuitry for 64 input signals plus one "dummy" channel input is presented in Figure 6.6. The prototype IC has only 16 channels and does not utilize an extra "dummy" channel. Each input voltage is converted to a current in an approximately linear manner via a resistor and a FET in series, though the FET is wide enough that its effective resistance is small compared to the resistor. The resulting current signals are sent into an array of identical FETs $\left(M_{1 A}\right.$ to $\left.M_{n A}\right)$ whose gates and sources are tied together, yielding an identical $V_{G S}$ for all the devices. The actual value of $V_{G S}$ is set by the transistor experiencing the greatest drain (i.e., input) current.

The $M_{n A}$ array of FET transistors all operate in the saturation region such 
that they demonstrate the following behavior [137]:

$$
I_{D}=\frac{1}{2} \mu C_{o x} \frac{W}{L}\left(V_{G S}-V_{t}\right)^{2}\left(1+\lambda V_{D S}\right)
$$

where $I_{D}$ is the drain current through the FET, $V_{G S}$ is the gate-to-source voltage, $V_{D S}$ is the drain-to-source voltage, $V_{t}$ is the threshold voltage, $\mu$ is the carrier mobility, $C_{o x}$ is the oxide capacitance, $W$ is the transistor width, $L$ is the transistor length, and $\lambda$ is the channel-length modulation. Since $V_{G S}$ is set and $I_{D}$ is determined by the input signal, the $I_{D}$ for each transistor establishes the corresponding $V_{D S}$ as per the relationship in Equation 6.7. Operation in the saturation region provides the $M_{n A}$ FETs with a large transconductance, $\frac{d V_{D S}}{d I_{D}}$, of around $1-40 \mu \mathrm{A} / \mathrm{V}$. Thus small differences in the input currents produce relatively large differences in the $V_{D S}$ of the $M_{n A}$ FETs, which in turn results in large differences in the $V_{G S}$ of the $M_{n B}$ FETs. Since the $M_{n B}$ array of transistors also operates in the saturation region, the drain currents $I_{\text {outn }}$ depend upon these $V_{G S}$ values in a square fashion as per Equation 6.7. However, all of the $M_{n B}$ FETs have their sources tied to a common current supply which is limited to about $30 \mu \mathrm{A}$. As a result, the FET with the largest $V_{G S}$ will take nearly the entire supply current and leave the remaining channels with virtually no current. In this manner the channel with the largest input voltage signal (i.e., the winner) will produce a $\sim 30 \mu \mathrm{A}$ output current and force the other output currents to zero. The series of output currents, $I_{\text {outn }}$, can therefore be used as logical bits to identify the winning channel. An encoder using pull-down logical circuits is used to produce a 4-bit address for the a 16-channel WTA or a 6-bit address for the 64-channel WTA.

Finally, the prototype WTA IC also includes a pseudo-state machine and the associated digital protocol. This is, of course, a preliminary version of the (true) state machine implemented in the final PETRIC and incorporates the WTA control and register read functions listed in Section 6.1 for the PETRIC state machine. 


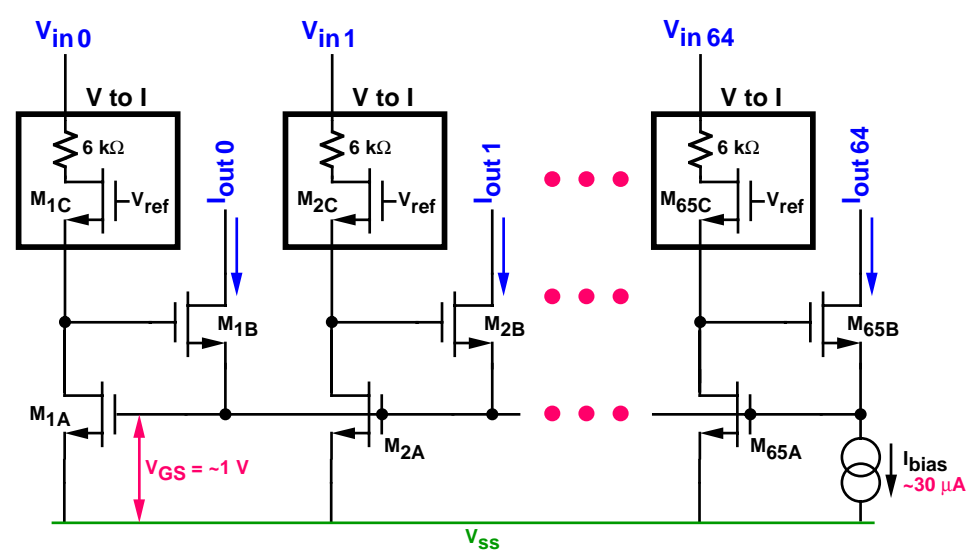

Figure 6.6: Simplified circuit diagram of the WTA for 64 input channels plus a dummy channel, though the design could be applied to most any number of channels. The channel with the largest input voltage will turn on its $M_{n B}$ FET the hardest and will take virtually all of the limited $30 \mu \mathrm{A}$ supply, leaving the other outputs with zero current.

\subsubsection{Prototype Performance}

The 16-channel prototype WTA IC successfully met its specifications with a few minor adjustments addressed further in Section 6.4.1. This evaluation was performed by connecting the WTA inputs to external signal generators and simultaneously observing the input signals, the analog output signal, and the address bits on a digital oscilloscope. To verify the correct identification of the winner channel via the address bits, a sizable dc voltage was placed on a given input channel and the address bit values noted. By gradually adjusting this dc voltage, it was also possible to observe the smallest voltage level that the WTA required to identify the correct winner signal. In order to observe the WTA with time-varying inputs, sinusoids were used and the winner typically switched back and forth between only two channels.

The IC correctly tracks the input signal with the greatest output and buffers that waveform to the analog output line. Further, the address of the winning signal is correctly generated within $50 \mathrm{~ns}$ of a change in the winning signal. Obviously this 


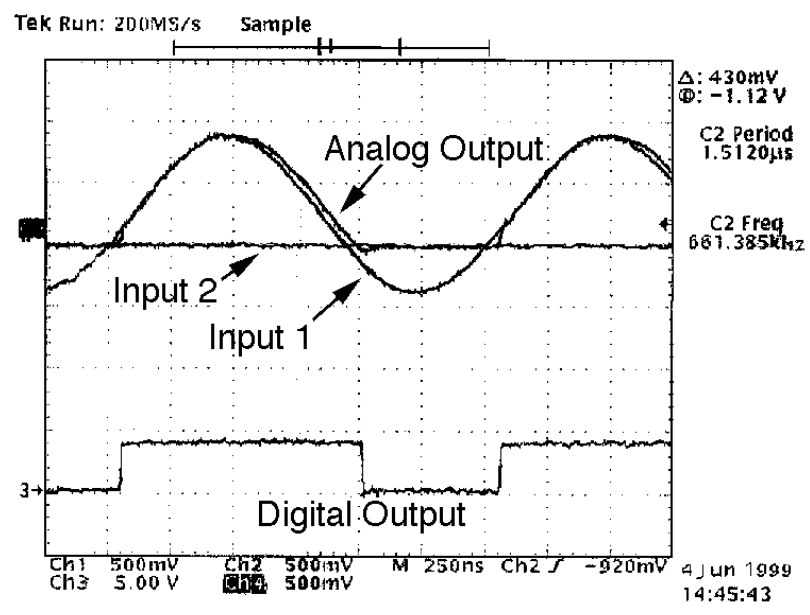

Figure 6.7: Oscilloscope traces demonstrating the prototype WTA performance. The three traces on top include Input 1 (a $661 \mathrm{kHz}$ sinusoid), Input 2 (a DC signal), and the analog output which correctly tracks the maximum signal presented by these two inputs. The other 14 inputs to the WTA are DC signals smaller than Input 2. The lower trace is a digital address bit that is high when Input 1 is the winner and low when Input 2 is the winner. The WTA is designed to have a first-order response and a Gain-Bandwidth product of $8 \mathrm{MHz}$.

switching speed is more than adequate when using peaking times on the order of $8 \mu \mathrm{s}$. An example of the output tracking and address identification is presented in Figure 6.7 for two input signals (the other 14 have lower voltages than the two shown) and a single address bit. There are some small differences between the winning input waveform and the WTA analog output, but these deviations tend to take the form of: (1) a short lag in the analog output catching up to a new winner or (2) a slightly delayed decrease in the analog output voltage as the winner decreases. Neither of these effects is severe, however, and neither interferes with the analog output correctly tracking the peak of the winner signal, which is the critical value necessary to achieve good energy resolution.

Due to the high gain of the WTA, a potential source of error is transistor 
mismatch. Measurements on several prototype ICs have shown that in the worst case an input signal must be $\sim 25 \mathrm{mV}$ above another signal in order to be selected as the winner. Since this is slightly smaller than the rms noise voltage expected at the inputs, however, it is entirely acceptable.

Although there are a number of errors in the digital protocol for the prototype WTA IC, it is possible to work around them and use the state machine as intended. The IC can be successfully placed in either WTA or multiplexer mode (the latter being wherein a specific channel is always connected to the analog output regardless of input signal levels), individual channels in the WTA can be turned on and off, and the state machine registers can be read out.

\subsection{Complete PETRIC Chip}

In addition the many challenges associated with developing and debugging the circuitry in the 16-channel prototypes of the front end and WTA, the process of designing and debugging a successful 64-channel IC that incorporates all of these functions in a single chip presents an entirely new set of difficulties and hazards. Obviously the amplifier, WTA, and state machine circuitry each need to be scaled up from 16 to 64 channels and then all of these elements must be placed together in a single IC. Not only is this a layout challenge, but it raises the issue of how to provide for testing and debugging of individual sections independently of the others. The low-noise, mixed analog/digital design of the PETRIC requires that certain traces be carefully shielded from others and that power and ground be carefully supplied throughout the IC. Finally, the corrections and design revisions suggested by the testing of the prototype ICs must be implemented, a significant task since these changes cannot be allowed introduce any new errors that may threaten performance. An overhead view of the complete 64-channel PETRIC layout is shown in Figure 6.8 


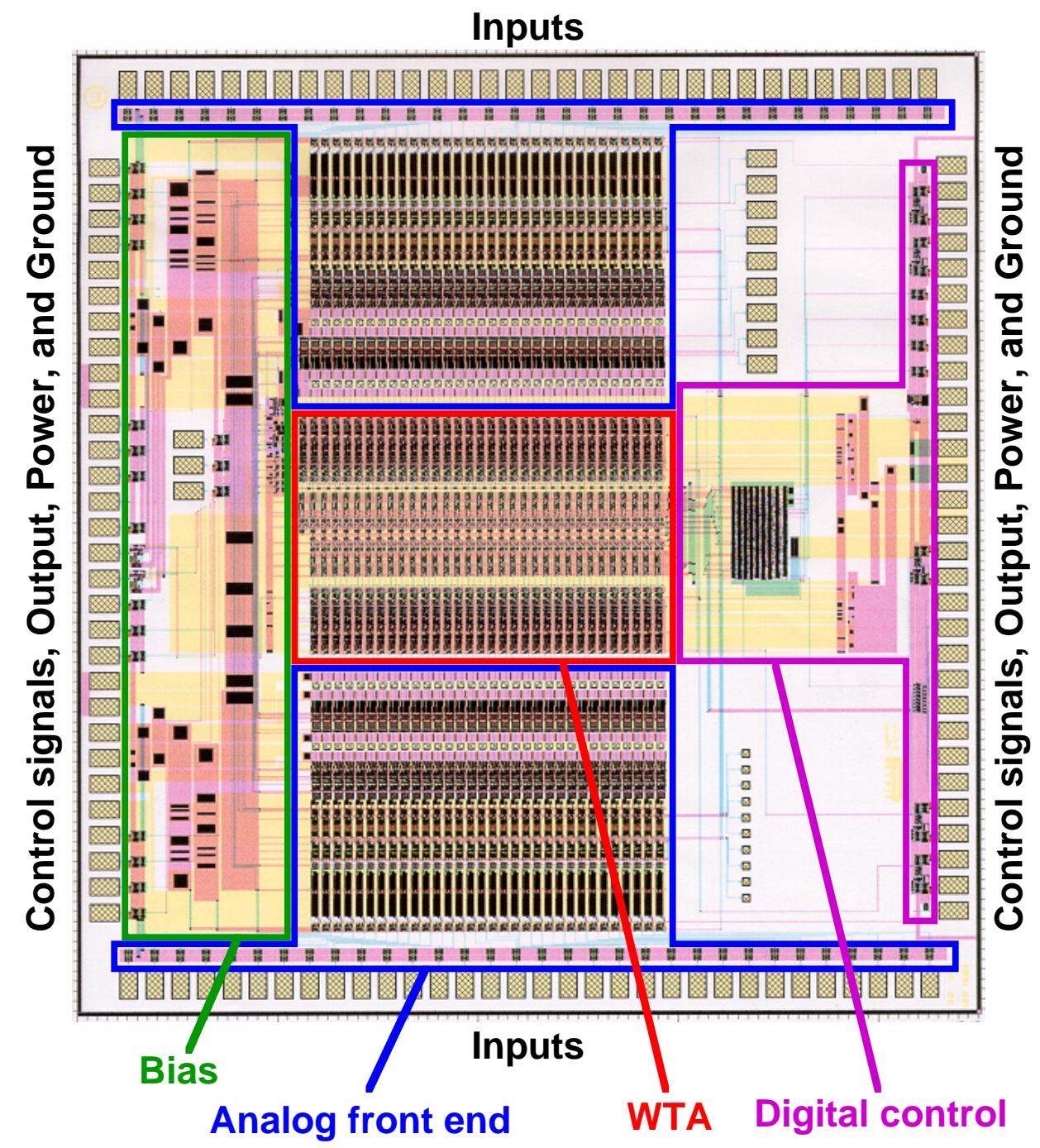

Figure 6.8: Overhead view of the PETRIC layout. Split evenly between the top and bottom are 64 analog input pads that are wirebonded to the input signal traces. Lining the left and right are a total of 60 pads that comprise the IC's input (e.g., digital protocol and externally-adjustable current sources), output (e.g., analog winner and 6-bit digital address), power, and ground signals. The sections outlined in blue indicate the analog front end channels, the section in red represents the WTA and associated encoder, the section in purple is the digital control, and the section in green indicates the bias circuitry. 


\subsubsection{Design Changes Suggested by Prototype ICs}

Based on the prototype studies with the 16-channel front end and WTA ICs, as well as an earlier 64-channel PETRIC precursor that nominally contained all circuit components but which failed to operate properly, numerous modifications and improvements were implemented in the PETRIC design. I participated in all of these design decisions, but have made the most significant contributions to those that pertain to the front end (items $3-6$ ). The fixes include:

- Perhaps most importantly, extreme care has been taken in the layout of the substrate contacts and in the power supply distribution to avoid cross-coupling between the digital logic and the sensitive analog inputs. The 64-channel PETRIC precursor oscillated catastrophically because the analog output lines and the digital signals coupled back into the preamp inputs through, in part, poorly designed power lines whose layout provided positive feedback paths to the preamp inputs. Such feedback renders the chip inoperable (the outputs oscillate uncontrollably between the power supply rails) and absolutely must be avoided. The power supply distribution was originally identified as the culprit by systematically eliminating all other possible positive feedback paths with comprehensive EMF shielding. In the final PETRIC design the power lines are much cleaner and robust (they are wider and better distributed so that they are less likely to experiencing fluctuations as the chip operates). Just to be safe, two separate power supply schemes were designed and fabricated. The only design tested thus far does not show any sign of oscillating (the outputs are never driven to the power supply rails) and hence this effort has proven successful.

- With these same motivations plus the goal of low noise in mind, the lowresistivity substrate has been fully exploited by plating the back of the IC with gold. This provides the best possible $-3.3 \mathrm{~V}$ power supply connection to the substrate and should therefore provide the best transistor behavior. The gold 
plating was not implemented to target a specific problem, but rather to offer a minor performance boost. It was noted, however, that this low-resistivity substrate connection should help to minimize the power supply concerns in the bullet point above.

- Front end channels with protection diodes and the transistor feedback mechanism for resetting the preamp have been implemented. Protection diodes do not harm noise performance in the prototype front end ICs (even though extra capacitive and current loading had originally been expected) and may provide some small measure of protection for the IC. I selected the transistor feedback mechanism not for its anticipated improvement in noise performance (it is indistinguishable from the externally-controlled current source mechanism for sinking the detector current), but rather because during experimental measurements it is empirically easier to keep the preamplifiers with that reset scheme out of saturation. The implementation of the these diodes and reset system has successfully yielded operational channels with essentially the same noise performance that I observed in all four types of prototype front end channelsabout $150 \mathrm{e}^{-}$rms at $2 \mu \mathrm{s}$ and $180 \mathrm{e}^{-}$rms at $8 \mu \mathrm{s}$. There has been no difficulty with keeping the channels out of saturation, and whether the protection diodes offer any substantial protection has not been studied.

- Individual control of each channel's preamp compensation current/reset time has been implemented (to an accuracy of 4 bits) because it became clear to me that chip-wide current compensation control would induce poor behavior in some channels. This has proven to be a major success. With the prototype front end ASICs it was necessary for the preamp compensation current to be set to the maximum current required by any of the channels, otherwise some channels would become saturated and essentially inoperable. This, however, meant that some channels experienced unnecessarily high compensation currents and therefore suffered higher electronic noise. The end result was that 
parallel noise in every channel was increased to nearly the worst value of any of the channels in the system. This is a significant concern because a given photodiode array may have a large range of leakage currents. In the final PETRIC design, however, the compensation for each channel is set individually and electronic noise is therefore minimized. Channels experiencing high load current still need a lot of compensation current and do demonstrate high parallel noise, but channels with low load currents can nonetheless simultaneously achieve low parallel noise.

- Current supplies have been increased in the shaper output amplifier to eliminate slew rate limitations. In the prototype front end ASIC the output waveform became distorted by slew rate limits for any input charge injection exceeding $3000 \mathrm{e}^{-}$(the rise of the output became increasingly triangular as signal levels increased). I identified the shaper as the problematic stage by observing that the preamp output signals sent to the shaper had no slew limits themselves, and this was further narrowed down to inadequate current supply in the shaper output amplifier by means of transistor-level HSPICE simulations. Naturally such slew rate limits are a problem, as signal levels in excess of $6000 \mathrm{e}^{-}$are anticipated. Distortion of the shaper output needlessly increases electronic noise by altering the peak pulse height of the output. In the PETRIC this slew rate limitation has been eliminated and inputs of up to $8000-9000 \mathrm{e}^{-}$suffer no distortion in the output waveform.

- A preamp bias voltage has been increased to prevent ringing in the preamp signal and thereby improve noise performance. This problem was identified by observing that the signals output by the prototype front end preamps experience unexpected ringing (immediately after the initial rise which occurs in response to an input charge). Transistor-level HSPICE simulations pointed to the need to increase the bias voltage of a particular node. I modified a prototype ASIC by adding additional wirebonds and a potentiometer to supply the 
suggested bias to the node in question. This eliminated the noise and slightly improved the electronic noise behavior. The final PETRIC design implements this fix and therefore does not incur the small noise penalty associated with the ringing.

- The $\sim 30 \mu \mathrm{A}$ limited current supply that the FETs fight for in the WTA is now derived from an internal current supply that is used as a logical threshold instead of form an external current supply. This improves the WTA switching speed when a new winner arrives and minimizes the worst case threshold for how much a signal must surpass the others before it is recognized as the winner. This improvement was implemented on the basis of transistor-level HSPICE simulations that were part of design process that scaled the WTA up from 16 to 64 channels while attempting to maintain the same performance. In the final PETRIC the identification of a new winner occurs within $150 \mathrm{~ns}$, compared to $50 \mathrm{~ns}$ in the 16-channel prototype. For both the prototype and the PETRIC, the winning signal must exceed other channels by about $25 \mathrm{mV}$ to be properly identified.

- The WTA dummy channel was added to prevent needless chatter on digital lines when no channel has a significant input. Based on the 64-channel IC which was a precursor to the PETRIC and which was essentially useless because it experienced positive feedback oscillations, it seemed prudent to minimize unnecessary switching on digital lines. Whereas in the prototype WTA IC random fluctuations on the input lines regularly cause the digital address bits to switch even in the absence of an input, in the PETRIC the dummy channel maintains silence on the digital bits when no input signal is presence. In theory this should also lower electronic noise by minimizing any signal injection into the output waveform.

- The PETRIC digital protocol is substantially different than that in the pro- 
totype WTA IC. While both protocols are supposed to implement the same logical function, the prototype system uses decoder-type logic which fail to recognize all commands properly. With creative sequencing of commands it is difficult but nonetheless largely possible to communicate with the IC. In the PETRIC, however, a synchronous state machine and a small asynchronous block to detect START/STOP events has been implemented. With the exception of a few bits which were accidentally switched (a problem that was easily corrected in software), this protocol works exactly as desired.

I have verified that these modifications provide the expected performance improvements in the PETRIC without introducing problematic new errors. The digital protocol does still retain a few minor errors, but these are easily correctable in software.

\subsubsection{Characterization of the PETRIC}

The gain of the PETRIC front end was measured using external injection capacitors. Small capacitors about $3 \mathrm{pF}$ in size were placed on a printed circuit board near a mounted PETRIC, and then wirebond connections were made between the PETRIC inputs and the capacitors. Signal generators were then used to deliver square waves to the other ends of the capacitors, allowing a known charge to be injected into the front end channels $(\mathrm{Q}=\mathrm{CV}$, where the capacitance includes both the known capacitance of the external capacitor as well as an estimate of the capacitance of the wirebond connection). Figure 6.9 presents the output waveforms for a single channel at $1 \mu$ s peaking time for a range of input charge injections from 600 to $12,000 \mathrm{e}^{-}$with the preamplifier set for maximum gain of $120 \mathrm{mV} / 1000 \mathrm{e}^{-}$. At this setting the gain is linear to within $1 \%$ for input charges from 750 to $4000 \mathrm{e}^{-}$, and linear to within about $5 \%$ in the $5000-7000 \mathrm{e}^{-}$range that is the expected signal level for $\mathrm{CsI}(\mathrm{Tl}) / \mathrm{Si}$ PIN photodiode detectors. This linearity calculation was made by collecting gain data (peak pulse height of the output waveform divided by the 


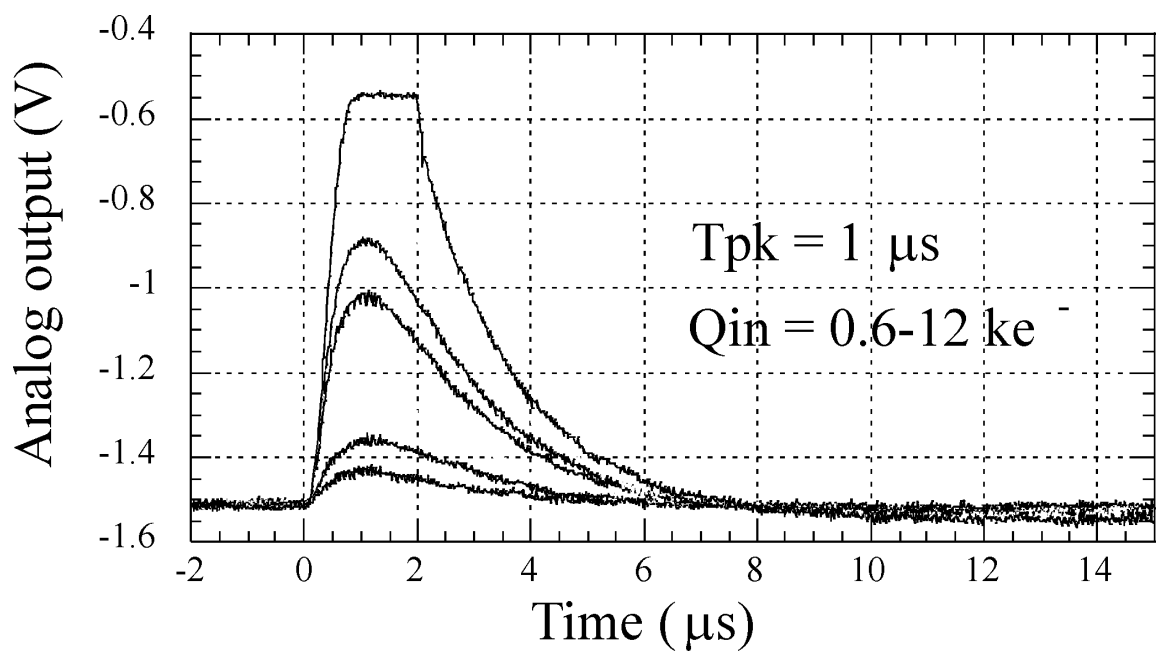

Figure 6.9: Waveforms produced by a single channel of the PETRIC front end in response to input charge injections ranging from 600 to $12,000 \mathrm{e}^{-}$. The shaper is set to a $1 \mu$ s peaking time and a gain of $120 \mathrm{mV} / 1000 \mathrm{e}^{-}$. The gain of the front end is reasonably linear until saturation occurs at input levels near $9000 \mathrm{e}^{-}$.

amount of charge injected into the front end) over the indicated input ranges fitting a straight line to the data, and noting the deviation from linearity. At around $9000 \mathrm{e}^{-}$the PETRIC output stage saturates. If greater gain linearity is desired for $\mathrm{CsI}(\mathrm{Tl}) / \mathrm{Si}$ PIN photodiode systems, it is a simple matter to lower the gain setting so that the signals of interest fall within the $1 \%$ gain linearity regime. The relative gain dispersion between different channels on the same IC is about $3 \%$ rms. Finally, the DC offset variation for the baseline voltage of different channels within the same IC demonstrates a standard deviation of $8 \mathrm{mV}$.

The peaking time of the PETRIC front end can be varied with external currents from 0.5 to $15 \mu \mathrm{s}$. Figure 6.10 displays shaper output waveforms with shaping times from 0.7 to $9 \mu \mathrm{s}$, all of which exhibit approximately the same peak height, the same rise time-to-fall time ratio of about 1:4, and a return-to-baseline with minimal undershoot. Peaking times closer to 0.5 or $15 \mu$ s produce similar 


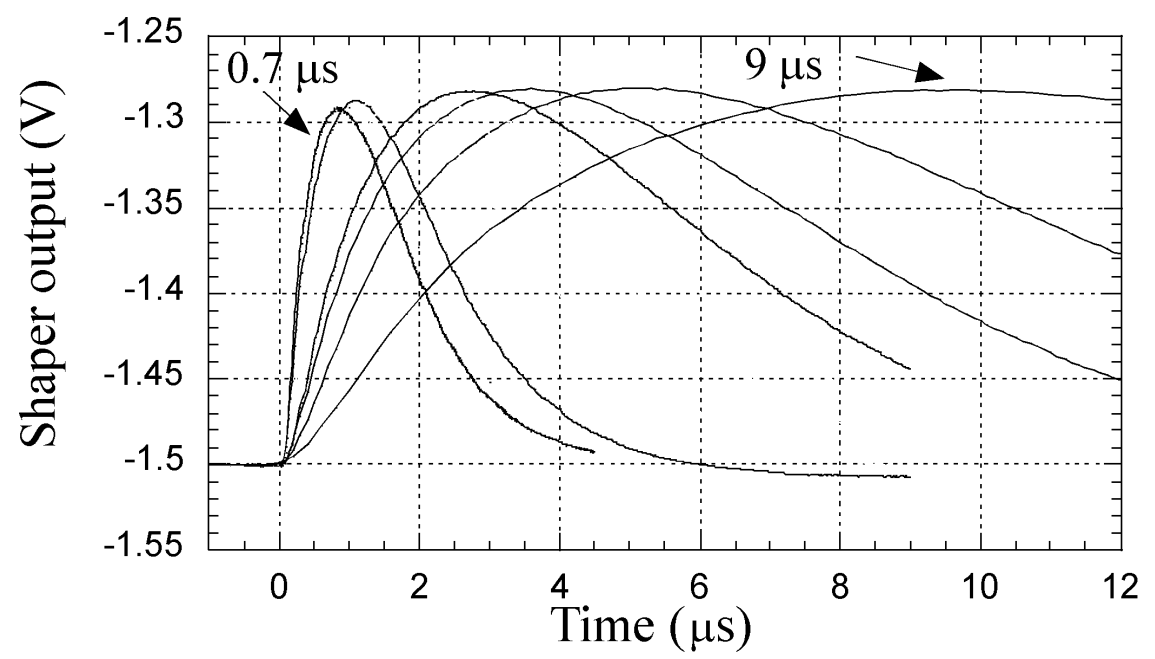

Figure 6.10: PETRIC front end waveforms with peaking times ranging from 0.7 to $9 \mu \mathrm{s}$. All waveforms exhibit approximately the same pulse height, the same rise timeto-fall time ratio of about 1:4, and the same minimal undershoot when returning to baseline.

waveforms but with slightly reduced pulse height and a small amount of sub-baseline undershoot as the waveform returns to its quiescent value.

Noise measurements performed on a test board (verses in a module) provide a lower bound for the noise performance of the PETRIC. Measurements made for channels with no input load (not even a wirebond connection to the test board trace) or with 3 or $6 \mathrm{pF}$ input loads are presented in Figure 6.11 as a function of shaping time. The results clearly demonstrate that series noise increases with increasing capacitance, that series noise is dominant below shaping times of about $4 \mu \mathrm{s}$, that $1 / \mathrm{f}$ increases with greater input loads, and that there is very little parallel noise. The last point is important because it indicates that virtually all of the parallel noisewhich is the dominant component at shaping times appropriate for $\mathrm{CsI}(\mathrm{Tl})$ - results from the leakage current in the photodiodes. Finally, note that with a $3 \mathrm{pF}$ input load (the expected capacitive load presented by the Si photodiodes) long shaping times 


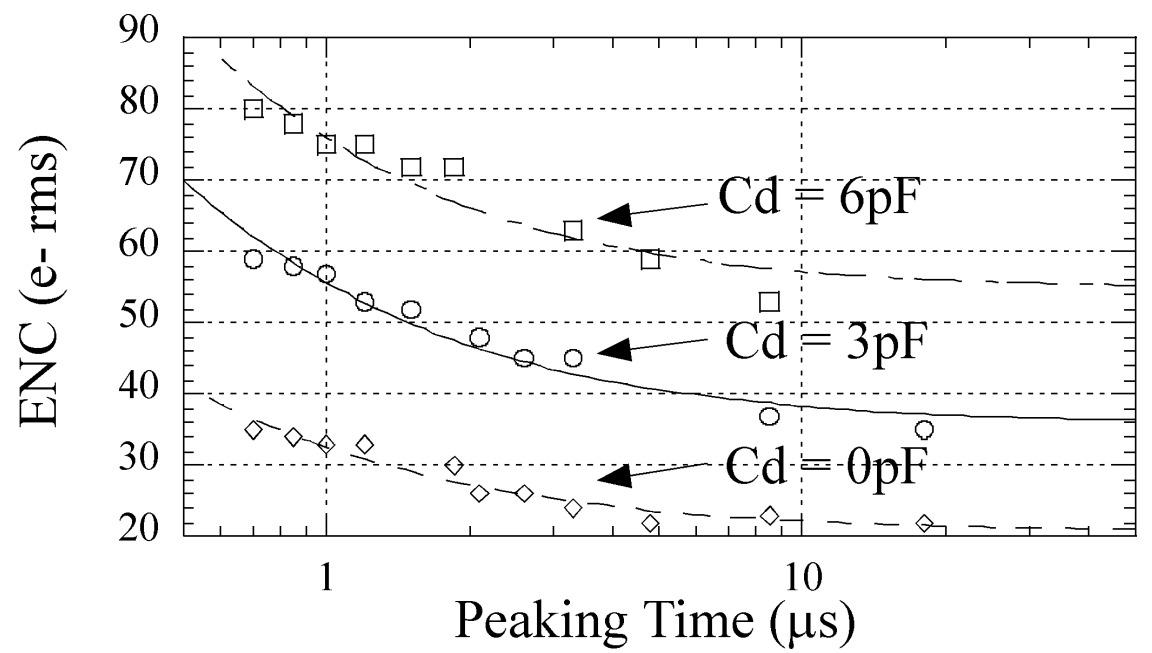

Figure 6.11: PETRIC electronic noise $(\mathrm{ENC}=$ equivalent noise charge $)$ versus peaking time for several capacitive loads. In the $0 \mathrm{pF}$ case the channel is not even wirebonded to the test board trace. Series noise dominates at short shaping times while $1 / \mathrm{f}$ noise dominates at longer shaping times. The overall noise performance is excellent and little parallel noise is apparent.

yield an electronic noise of less than $40 \mathrm{e}^{-} \mathrm{rms}$, which is far below the anticipated detector system electronic noise of $\sim 180 \mathrm{e}^{-}$rms. Thus the leakage current and $1 / \mathrm{f}$ contributions from the photodiode load are ultimately a huge concern (justifying the effort spent developing custom, low-noise photodiodes!).

The WTA section of the PETRIC circuitry successfully selects the input channel with the highest voltage and connects it to the analog winner buffer in less than 150 ns. Figure 6.12 displays the WTA analog output plus two digital address bits as the WTA responds to two input pulses offset in time by about $2.5 \mu$ s and presented on two different channels. The WTA correctly responds by continuously tracking the input signal with the greatest voltage and flipping the address bits accordingly. The DC dispersion of the WTA inputs in the same IC has been measured at $20 \mathrm{mV} \mathrm{rms}$, which dominates relative to the $8 \mathrm{mV}$ rms dispersion generated by the front end. These channel-to-channel variations in DC voltage are small enough 


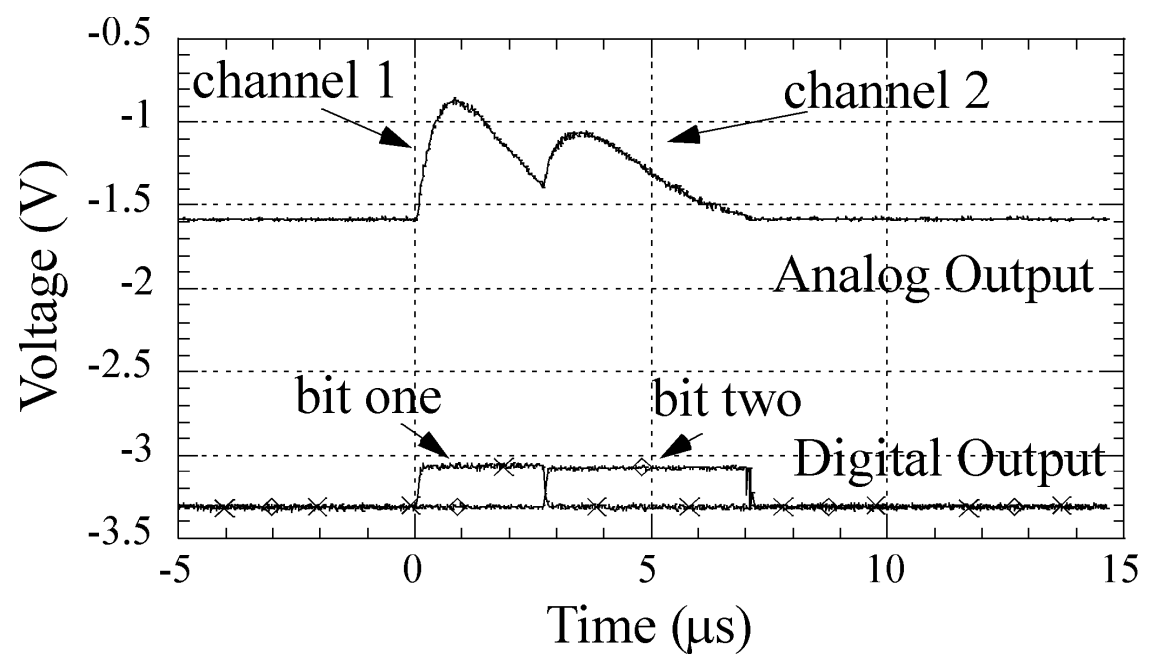

Figure 6.12: Performance of the WTA circuitry in the PETRIC. Two input charges are injected into separate front end channels, producing two pulse waveforms which excite the WTA. The first charge injection, which is slightly larger, occurs at time = $0 \mu \mathrm{s}$, while the second is injected at about time $=2.5 \mu \mathrm{s}$. The analog output of the WTA tracks the first input waveform until the second pulse becomes larger, at which point the analog output tracks that signal and the digital address bits switch. Bit 1 is high when channel 1 is the winner, bit 2 is high when channel 2 is the winner, and neither is high when both signals are at baseline values (and the threshold channel is therefore the winner).

to be of little consequence to the WTA, however, because the $65^{\text {th }}$ "dummy" channel can easily be set so that it is always selected as the winner when no input signals are present.

Taken as a whole, these results demonstrate that the PETRIC successfully meets its design specifications. This ASIC provides a highly compact, high performance, flexible readout scheme for modular compact gamma cameras employing low capacitance, low leakage current photodiode detectors at moderate event rates. Since the PETRIC front end and WTA both perform extremely well in a testing environment and the prototype system described in Chapter 4 shows the successful integration of custom photodiodes and ASIC readout, the only major performance 
issue that remains is the question of noise behavior (and hence energy resolution) in the final module environment with the 64-pixel photodiode input loads. This is addressed in Chapter 7 along with the engineering details behind the integration of the PETRIC into complete modules. 


\section{Chapter 7}

\section{Complete 64-Pixel Imaging Modules}

The whole is greater than the sum of the parts.

- Proverb

This chapter serves to pull the entire thesis together into a coherent wholeI explore the final design, construction, and performance of 64-pixel imaging modules. These modules are the critical building blocks for complete, modular, compact gamma cameras appropriate for breast cancer imaging, and the successful performance of these final modules allows for the implementation of a wide variety of camera shapes and sizes. Modules employing 64 pixels provide enough area to make modular cameras feasible and straightforward, yet they remain small enough to offer a great deal of geometric flexibility. While substantial multiplexing of information from the 64 channels is required, the multiplexing remains manageable with IC readout and the dead time is still low.

In addition to the design, assembly, and basic characterization of these final modules, I have concurrently developed the basic design for a complete, planar, 16-module compact gamma camera intended for breast cancer imaging (Section 7.4). 
I substantiate the advantages of this camera for scintimammography with the aforementioned basic performance measurements of the 64-pixel modules (Section 7.5), demonstrating that the 12-pixel prototypes described in Chapter 4 have been successfully scaled up to 64 elements. These results, coupled with both the 12-pixel behavior observed in those early prototypes and the Chapter 5 simulation studies of breast cancer imaging with compact, discrete gamma cameras, provide a preliminary and encouraging characterization of scintimammography cameras utilizing this technology.

Figure 7.1 summarizes the "big picture" system design for the 16-module camera which will ultimately utilize the 64-pixel modules described and characterized in this chapter. This conceptualization of the final camera sets the framework for the design decisions described in the sections that follow. A patient or other radionuclide distribution is imaged with the 16-module camera, which employs a parallel hole collimator to provide directional information. The camera produces the following outputs: (1) the peak voltage of the analog shaped output pulse which was generated by the detector/readout system in response to a gamma ray interaction, (2) the 10-bit digital address indicating which of of the 1024 pixels was the site of the gamma ray interaction, and (3) a timing strobe which signals the computer to acquire (via an acquisition board) the present pulse height voltage and digital address bits. The information for the event in question is then incorporated into the set of image data. Energy discrimination and any other data processing that is desired take place within the software. Only analog output pulses which exceed a certain (adjustable) threshold will trigger the timing pulse, however, so low-energy noise pulses are eliminated before ever reaching the computer. Since one of the data lines supplied to the acquisition computer is to carry the peak pulse height of the shaped analog waveform, the camera electronics obviously must include peak detect circuitry which determines and then holds this peak voltage until it is acquired.

The truly innovative aspects of this thesis project - and especially the final module and camera designs - revolve around making the imaging device as compact 


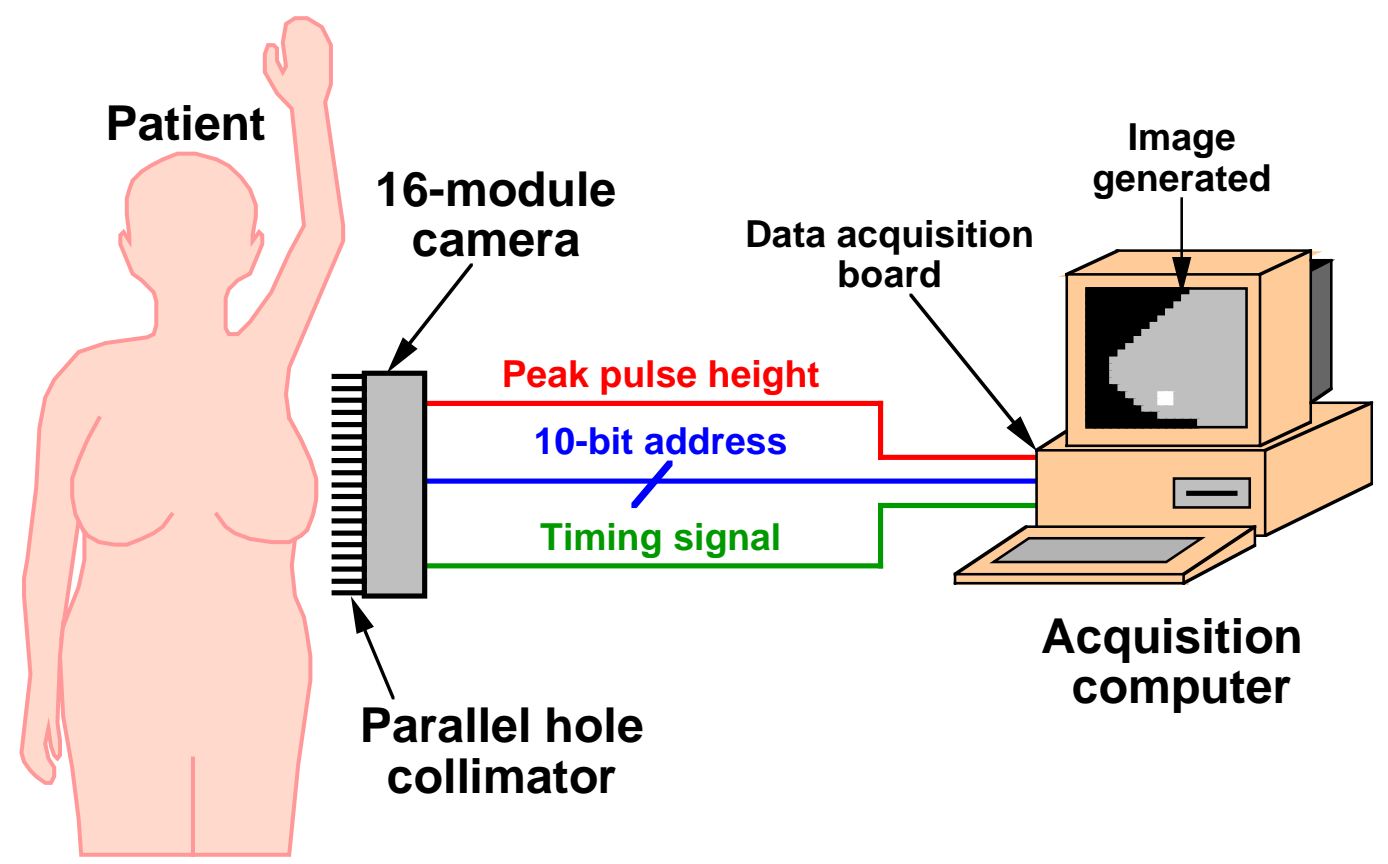

Figure 7.1: System level diagram for the 16-module camera indicating the "big picture" for how the camera is to be employed. The camera, which consists of 16 64-pixel modules and therefore has 1024 pixels, images a patient or other radionuclide distribution and produces outputs which include: (1) the peak voltage of the shaped output pulse, (2) the 10-bit address which identifies the pixel of interaction, and (3) a timing signal which triggers the acquisition of data by the computer. The acquisition computer then compiles the information into a planar image. 
as possible in order to optimize it for breast imaging. This, of course, allows imaging distance to be reduced and makes possible imaging views typically infeasible with an Anger camera. Further, compact design introduces the possibility of using multiple cameras simultaneously to collect more information in the same amount of time and to provide rudimentary information on tumor location in three dimensions.

The two critical components that make this scintimammography camera technology feasible for the first time are the custom IC readout and the low-noise Si PIN photodiodes. The former provides extremely dense processing of 64 pixel signals

per $4.5 \times 4.8 \mathrm{~mm}^{2}$ IC as described in Chapter 6 , while the latter replaces the bulky and expensive PMTs used in traditional Anger cameras. The notion of replacing the PMTs in scintillation cameras with photodiodes has long been contemplated, but the low-noise photodiodes described in this thesis are the first with sufficiently low room temperature leakage current to provide adequate energy resolution for this application. The final 64-pixel photodiode arrays used in the camera modules are described in Section 7.2.

While the ASIC and the photodiode arrays are the technologies that make this camera concept feasible, there remains the need to package all of the camera components in a manner that maintains compactness, introduces little or no dead area in the imaging FOV, and allows all components to interface together and operate properly. This is straightforward in concept, but challenging in practice. As a further constraint, the design must incorporate the unique camera design knowledge gained from the Monte Carlo simulations presented in Chapter 5. The final 64-pixel modules and gamma camera architecture that I optimized for scintimammography are covered in Sections 7.3 and 7.4, respectively.

\subsection{Gamma Camera Design Parameters}

In designing the final 64-pixel modules and the related 16-module camera, compactness is obviously not the only goal. Not only did I also carefully consider 
my Monte Carlo scintimammography simulations, but I paid specific attention to matching or improving upon important characteristics offered by traditional Anger cameras. Since Anger cameras have demonstrated some success in scintimammography clinical trials (Section 2.4.1), it is reasonable to use them as a preliminary yardstick for compact, discrete gamma cameras targeting this same application. The most important design parameters in addition to compactness include:

- Field of view. Our compact, discrete gamma camera design has a smaller field of view than a traditional full-body Anger camera, but with organ-specific applications such as breast imaging this is not a drawback. The $9.6 \times 9.6 \mathrm{~cm}^{2}$ coverage offered by the 16-module design should be sufficient for scintimammography, but if more coverage is desirable the modular design makes the construction of larger cameras straightforward.

- Dead space. In an Anger camera the dead space at the periphery of the device is typically several inches wide, while with our compact camera the dead space need only be as thick as the shielding surrounding the modules-potentially less than a quarter inch. The shielding in question needs to protect against stray gammas, electromagnetic fields (EMF), and light.

- Energy resolution. Traditional Anger cameras demonstrate an energy resolution of about $9 \%$ fwhm for the $140 \mathrm{keV}$ emissions of ${ }^{99 m} \mathrm{Tc}$. At room temperature my 12-pixel $\mathrm{CsI}(\mathrm{Tl}) / \mathrm{Si} \mathrm{PIN}$ photodiode detectors exhibit a resolution of less than $11 \%$ fwhm, with the potential to improve to $7-8 \%$ fwhm if cooled to around $5^{\circ} \mathrm{C}$. However, the Monte Carlo simulations presented in Chapter 5 suggest that $15 \%$ fwhm is adequate for scintimammography in geometries where there is minimal scatter (such as the craniocaudal view). In other geometries improved energy resolution may be more important.

- Intrinsic spatial resolution. Traditional Anger cameras have an intrinsic spatial resolution near $3.5 \mathrm{~mm}$ fwhm, while that of discrete scintillation cameras 
is almost entirely determined by the pixel size. I have chosen a pixel size of $3 \times 3 \mathrm{~mm}^{2}$ because it slightly improves upon the Anger camera spatial resolution but maintains a manageable density of pixels to read out. This pixel size is also supported by the Chapter 5 Monte Carlo simulations.

- Collimator design. The design of the collimator(s) for Anger and compact gamma cameras are very similar in that high sensitivity is necessary to image effectively in an event-starved environment. Compact, discrete cameras, however, have the advantage of offering higher collimator spatial resolution for the same collimator design because of the reduced imaging distance. Further, cameras with square detector pixels can potentially use square rather than hexagonal collimator holes. Again in accordance with the Monte Carlo simulations, I have chosen several high sensitivity hexagonal hole lead collimator designs.

- Maximum event rate. A scintimammography camera must be able to process events at $1-10 \mathrm{kHz}$ to handle the 100,000 s of events collected during a typical 10 minute study. The modular, discrete gamma camera architecture presented in this thesis can easily achieve this goal. It is straightforward to implement the modular design such that only a single module suffers dead time during an event (because the IC is busy processing the electric signals), leaving the other modules active. Further, the discrete nature of the $\mathrm{CsI}(\mathrm{Tl})$ scintillator crystals prevents the finite scintillation decay times from posing a camera-wide dead time problem. In conventional Anger cameras the entire system is affected by the dead time resulting from one event (because of the finite scintillation decay time in the single $\mathrm{NaI}(\mathrm{Tl})$ slab), but most modern Anger cameras now implement a multiple trigger zone design so that dead times are more localized and are not camera-wide $[139,140]$. 


\subsection{4-Pixel Low-noise Photodiode Arrays}

The 12-pixel prototype low-noise PIN photodiode arrays are described in detail in Section 4.2.2, and the goal in implementing the 64-pixel arrays was simply to replicate the prototype photodiode performance on a larger scale. This is challenging in part because if the yield for individual pixels is $y$, then the yield for perfect 12pixel arrays is $y^{12}$ and that for 64 -pixel arrays is much smaller at $y^{64}$. Since the pixel yield, $y$, for the 12-pixel prototype arrays is only $\sim 80 \%$, this clearly presents a significant problem $\left(y^{64}=0.000063 \%\right.$ !). In order to increase the yield such that $y^{64}$ is acceptably high (i.e., at least 30-40\%) for the final arrays, a new process for depositing and annealing the phosphorus-doped polysilicon was developed [141]. While the old process involved deposition at $650^{\circ} \mathrm{C}$ and no annealing, the new procedure requires deposition at $500^{\circ} \mathrm{C}$ and annealing at $600^{\circ} \mathrm{C}$. My leakage current measurements demonstrate that the exposure to less extreme temperatures increases the photodiode yield, $y$, to $>98 \%$ and thus the array yield, $y^{64}$, to $>27 \%$. The leakage current of the photodiodes remains unaltered, but as an added performance bonus the resistivity of the polysilicon is reduced by almost an order of magnitude, hence there is less parasitic resistance in the supply path for the $50 \mathrm{~V}$ bias voltage.

A 64-pixel Si PIN photodiode array with $3 \times 3 \mathrm{~mm}^{2}$ elements is pictured in Figure 7.2. A series of four guard rings run around the perimeter of the array (but not around individual photodiodes) to protect against stray leakage current. The array is designed to maintain a $3 \mathrm{~mm}$ pitch between pixels when butted up against other arrays, so edge and corner pixels are slightly smaller than central pixels. The corners of the photodiode array are cropped slightly in order to allow space for small wires to carry the $50 \mathrm{~V}$ bias around the edge of the array to the backside.

The final photodiode arrays were fabricated on Si wafers with 7 arrays per wafer. Dicing the arrays from the wafers without damaging them was challenging because of their delicate nature and non-square shape (namely the missing corners), requiring that I work with multiple vendors to develop non-destructive techniques. 


\section{Si PIN photodiode array}

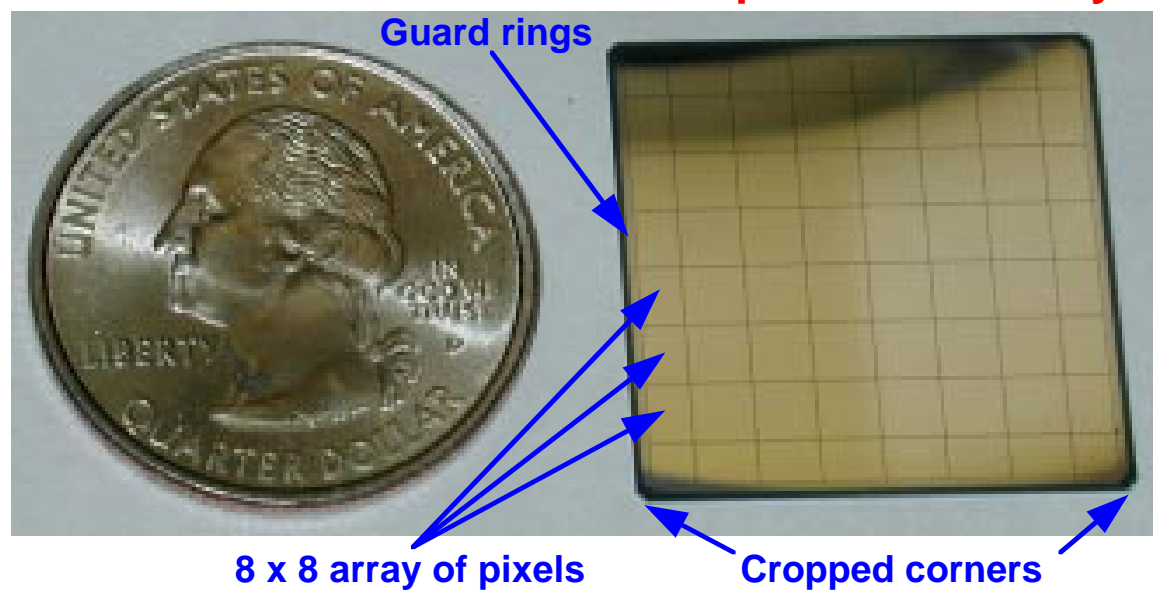

Figure 7.2: Low-noise Si PIN photodiode array consisting of $643 \times 3 \mathrm{~mm}^{2}$ pixels, with a quarter (24 $\mathrm{mm}$ diameter) for scale. The side shown is the patterned p-layer, while the backside is the unpatterned, light-sensitive n-layer.

Because laser cutting overheated the arrays, saw dicing with a total of 8 cuts per arrays was implemented. The application of a protective layer of photoresist that was only removed after the cuts were complete was successful in offering some protection from airborne debris.

I designed a probe card (Rucker and Kolls, Inc., Milpitas, CA) to measure the electrical properties of the final photodiode arrays. The probe card provides an array of 65 precisely-positioned tungsten tips which demonstrate excellent planarity, and therefore the tips can be lowered onto a naked Si photodiode array and make contact with the 64 pixels and guard ring simultaneously. The backside of the photodiode array rests upon a metal surface with a $50 \mathrm{~V}$ supply connected to it, effectively biasing the photodiodes. The 65 output signals from the probe card are then read out sequentially by multiplexing them to a high-input impedance meter which can measure currents down to the pA range. All measurements are made at room temperature in the dark under a thick, black cloth. My results are summarized 


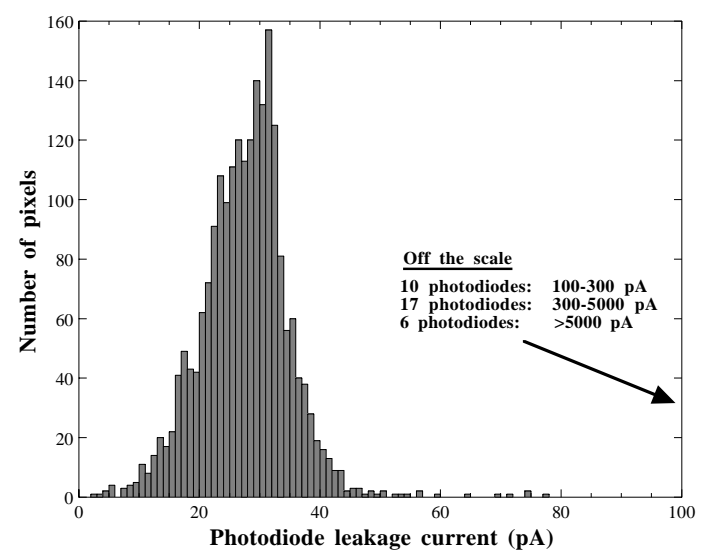

Figure 7.3: Histogrammed leakage current values for photodiodes in the 34 64-pixel arrays diced from the 5 most promising wafers. Histogram bins are $1 \mathrm{pA}$ wide. Photodiodes are $3 \times 3 \mathrm{~mm}^{2}$ in size and are in the dark at room temperature under a $50 \mathrm{~V}$ bias. The pixels with leakage current less than 100 pA (2143 of 2176) demonstrate an average current of $28 \pm 7 \mathrm{pA}$.

in Figure 7.3 in the form of a histogram of the leakage currents demonstrated by the pixels in the 34 arrays diced from the 5 most promising arrays. The "good" pixels (those with less than $100 \mathrm{pA}$ leakage current) demonstrate an average current of $28 \pm 7 \mathrm{pA}$, about an order of magnitude better than the best commercial photodiode arrays presently available. The guard rings of all 34 arrays exhibit an average current of $1.7 \pm 0.4 \mathrm{nA}$.

Using the $100 \mathrm{pA}$ metric, the yield for individual photodiode elements is 2143 of 2176 , or an impressive $98.5 \%$. This provides an adequately high $y^{64}$ of $38 \%$, which is consistent with our observed yield of $41 \%$ (14 of 34) flawless arrays. If one "bad" (i.e., high leakage current) pixel is acceptable, then the array yield increases to $74 \%$ ( 25 of 34 ). In addition to the high yield and excellent leakage currents, I verified that the photodiodes also demonstrate the expected $3 \mathrm{pF} /$ pixel capacitance (measured with an RLC meter) and $>80 \%$ quantum efficiency for $540 \mathrm{~nm}$ light (when coated with a $\operatorname{CsI}(\mathrm{Tl})$-optimized anti-reflective ITO layer $679 \AA$ thick as mentioned 
in Section 4.2.2).

The photodiodes are, however, extremely sensitive and can easily be destroyed. Their handling and incorporation into the compact gamma camera modules therefore requires great care and the use of specific epoxies. This is covered in more detail in Section 7.3. Nonetheless, this innovative photodiode technology has proven extremely successful and is a critical component not only in the compact gamma camera presented in this thesis, but also (in slightly different form) in the prototype devices in reference [94] and in the Digirad Corporation Model 2020tc Imager $^{T M}$ compact gamma camera.

\subsection{4-Pixel Module Design}

In Figure 7.4 I present my final design for 64-pixel compact imaging modules. The major components include the collimator, $\mathrm{CsI}(\mathrm{Tl})$ array, photodiode array, and readout IC. Also key are the two custom printed circuit boards (PCBs) that (1) offer electrical connections to the photodiodes, (2) support wirebond connections to the PETRIC, (3) house the surface mount bypass capacitors and EMF shielding necessary for PETRIC operation, and (4) provide the surface mount connectors used to plug the module into a motherboard when constructing an array of modules. The module design is relatively straightforward, but some innovation and experimentation were nonetheless required because of conflicting constraints. The chief concerns I strove to simultaneously address were: (1) the maximally compact design, (2) a large number of complex electrical connections, (3) a reliable and stable imaging device, and (4) an assembly procedure which does not harm any delicate components (most notably the PETRIC or the photodiode array). These issues are addressed further in the sections that follow. 


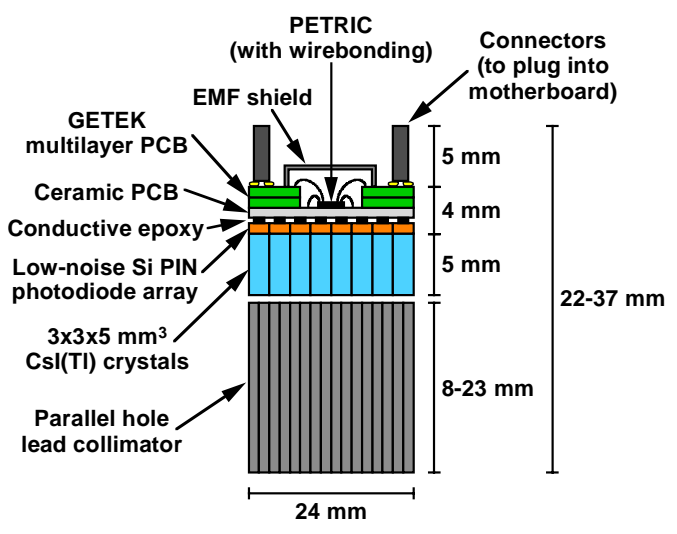

(a)

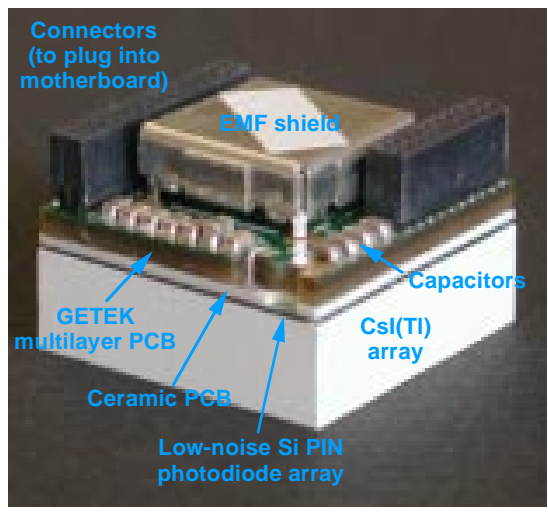

(b)

Figure 7.4: A complete 64-pixel CsI(Tl) scintillator/Si PIN photodiode imaging module with custom IC readout. (a) Diagrammatic cross section indicating the key components. (b) Photograph of an actual module (without the collimator). The total depth of the module depends on the collimator design but is less than $4 \mathrm{~cm}$ (though total camera depth is slightly greater than the module depth).

\subsubsection{Printed Circuit Boards}

I designed the custom PCBs as two distinct boards that are carefully aligned and glued together to form one assembly that provides all necessary connections to both the photodiode array and the PETRIC. Ideally the PCBs would be fabricated as a single multilayer board to cut down on the number of assembly steps, with ceramic as the substrate because of noise concerns which are described later in this section. This board would be quite complex, however, and at the time of this thesis, PCB fabrication technologies are not up to the challenge of accomplishing this with ceramic. Work in the coming years, however, may very well make use of such designs as the ability to fabricate multilayer ceramic boards advances. Figure 7.5 is a photograph of the 64-pixel module components - including the two PCBs-before any assembly has taken place.

The first board is mounted to the photodiode array and routes the detector 


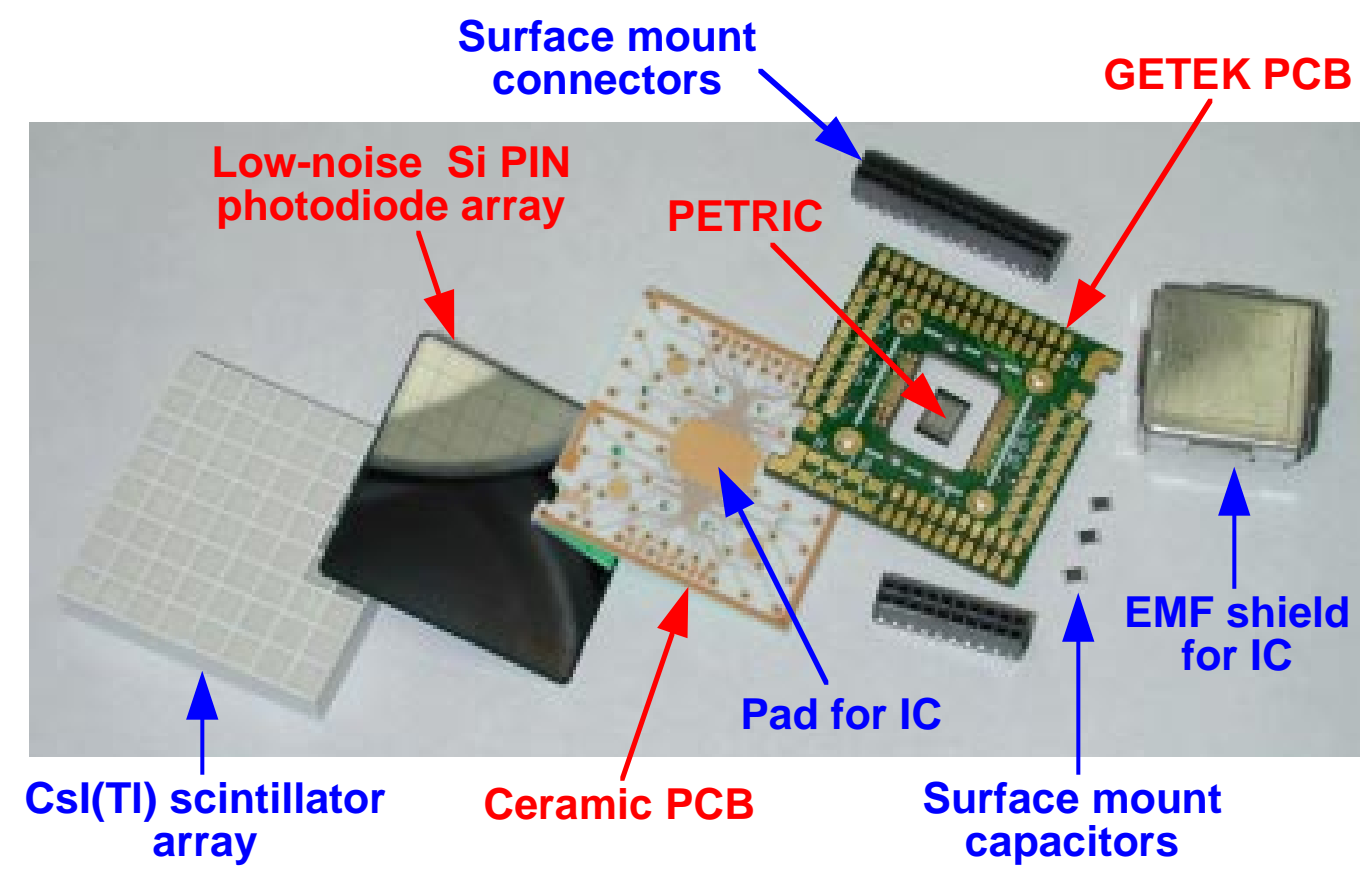

Figure 7.5: Key components (unassembled) in a 64-pixel modules. Those that are custom designed are indicated in red and include, in addition to the photodiode array and the PETRIC, two key PCBs. The ceramic circuit board connects (via conductive epoxy) to the photodiode array on one side and to the PETRIC on the other. The board's primary electrical purpose is to route the detector signals from the photodiodes to the wirebonded PETRIC inputs. The multilayer GETEK (General Electric Electromaterials, Coshocton, $\mathrm{OH}$ ) board also makes wirebond connections to the PETRIC and routes its output and control signals while providing space for (1) bypass capacitors, (2) an EMF shield for the PETRIC, and (3) surface mount connectors by which the module can be plugged into a larger motherboard. 
signals to the PETRIC, which sits on the other side. Because this PCB houses the photodiode array, the PETRIC, and the sensitive input lines, it must have excellent electronic noise properties. Ceramic is therefore employed because of its superior $1 / \mathrm{f}$ noise behavior. In addition, ceramic is a good thermal expansion match for the Si photodiode arrays and offers good thermal conductivity to help dissipate heat. Multilayer ceramic boards can be fabricated, but restrictions in the layer-to-layer spacing make them infeasible for this application. As such, a straightforward doublesided ceramic board with a coverlay (i.e., insulating layer) pattern on the photodiode side is employed.

The second board houses all the surface mount components, routes the PETRIC output and control signals, and provides a ground plane in the lower half of the board to help shield the sensitive input lines on the ceramic board from EMF radiation. Routing is something of a challenge because a large hole must be left in the center of the board to provide a bay into which the PETRIC is placed. This board must be multilayer, so GETEK is employed as the substrate because it offers this capability while providing better 1 /f noise behavior than the more standard FR-4 (but worse than ceramic). The final design of this board calls for 6 distinct layers.

\subsubsection{Assembly Procedures}

The procedures I developed for assembling complete modules begin with the custom PCBs, which are carefully glued together. The PETRIC is attached to the IC pad on the ceramic board with conductive epoxy and then wirebonded to both boards. The 64 input lines from the ceramic board are bonded to the PETRIC, as are the 51 output, control, and power/ground lines on the GETEK board. This, too, required that I work with multiple vendors to develop a workable bonding scheme, an effort which included modifying both my original custom PCB designs and my original order for assembling module components. In addition to the wirebonding, the surface mount capacitors, surface mount connectors, and EMF shield are soldered 
into place (all the while not disturbing the fragile wirebonds).

This then leads to the photodiode array, which is difficult to properly assemble because it is naked silicon and thus extremely fragile. Further, because low leakage current behavior is desired, the addition of any contaminate ions is unacceptable. I therefore selected three low ionic content epoxies - each with very specific characteristics - for use with the photodiodes:

- conductive epoxy, which electrically connects the 64 pixels to the ceramic circuit board and which must be very carefully applied with a custom stencil

- low viscosity underfill epoxy, which passivates the part of the photodiode surface between the array and the ceramic board that is not already covered with conductive epoxy, thereby protecting the photodiode surface and providing additional mechanical strength

- transparent coupling epoxy, which attaches the $\mathrm{CsI}(\mathrm{Tl})$ array to the lightsensitive side of the photodiode array.

In order provide the photodiodes as much protection as possible against this last epoxy, a polyamide passivation layer is first applied to the sensitive photodiode backside as a physical barrier to ions. This layer is transparent and does not noticeably decrease quantum efficiency.

During the various steps wherein the application of these epoxies is performed, wirebond connections are made to the guard ring and to the $50 \mathrm{~V}$ backside of the photodiode array. Obviously these bonds need to be made after the array is attached to the circuit board assembly but before the photodiodes are completely coated with epoxy and the silicon made inaccessible. Once these bonds are complete and the epoxies are all applied (using various assembly jigs), module assembly is complete. The final product (pictured in Figure 7.4b) includes everything from the $\mathrm{CsI}(\mathrm{Tl})$ crystals to the connectors, hence the only other components required are a collimator and a board with mating connectors that can control the module. 


\subsubsection{Interface and Control}

The requirements for controlling and interfacing with a module include management of the 36 output, control, and power/ground signals sent to and from the GETEK board via the two connectors offering a total of 50 pins (power and ground are duplicated on multiple pins). This includes:

- $-3.3 \mathrm{~V}$ power

- ground

- $50 \mathrm{~V}$ photodiode backside bias

- $-1.65 \mathrm{~V}$ photodiode guard ring bias

- the analog winner

- 6-bit address of the winner plus 2 error detection bits

- 2 calibration test pulse lines

- serial data and serial clock $\mathrm{I}^{2} \mathrm{C}$ lines

- PETRIC enable and reset signals

- current signal controlling preamp reset time/leakage current compensation

- 2 current signals controlling the shaper rise and fall times

- current signal controlling the WTA "dummy" channel threshold

- limited current supply that individual channels "fight for" in the WTA.

The address and error bits need to be converted from current signals to ECL (emittercoupled logic) to TTL (transistor-transistor logic), while the various current control signals require an easy means of adjustment via potentiometers in order to control PETRIC behavior. A block diagram of some of the basic routing for the signals 
listed above which is implemented for the 16-module camera is presented later in this chapter in Figure 7.9.

Further, in order to implement computer acquisition of the image dataincluding both maximum pulse height and the address of the pixel in which the pulse was generated-peak detect circuitry is employed. I designed this circuitry so that when it detects a pulse peak above a reasonable (and adjustable) noise threshold, it generates a read strobe that activates the computer data acquisition board. This board then latches in the address bits and performs analog-to-digital conversion of the peak value of the analog winner. The peak detect circuitry is presented in more detail in Section 7.4.1.

\subsection{Complete 16-Module Camera Design}

The conceptual design for the 16-module scintimammography camera-the culmination of the compact single photon technology described in this thesis - is presented in Figure 7.6. As has been described, 16 individual modules are plugged into a motherboard that controls the modules and interfaces with a computer. The result is a 1024-pixel camera covering an area of $9.6 \times 9.6 \mathrm{~cm}^{2}$. I designed the motherboard such that electronics requiring board space in excess of the imaging area are located on one - and only one - side of the imaging modules, preserving the camera's ability to gain close access to the breasts and axillary nodes.

\subsubsection{Camera Hierarchy and Motherboard Design}

Since the 64-pixel module design has already been discussed at length, the only part of the camera hierarchy that has yet to be addressed in detail is the motherboard design. As is indicated in Section 7.3.3, each module requires a substantial number of control signals and produces both an analog winner output and 8 digital outputs ( 6 address plus 2 error checking bits). I designed the motherboard schematic to accommodate these signals for all 16 modules and, in the case of the output bits, 


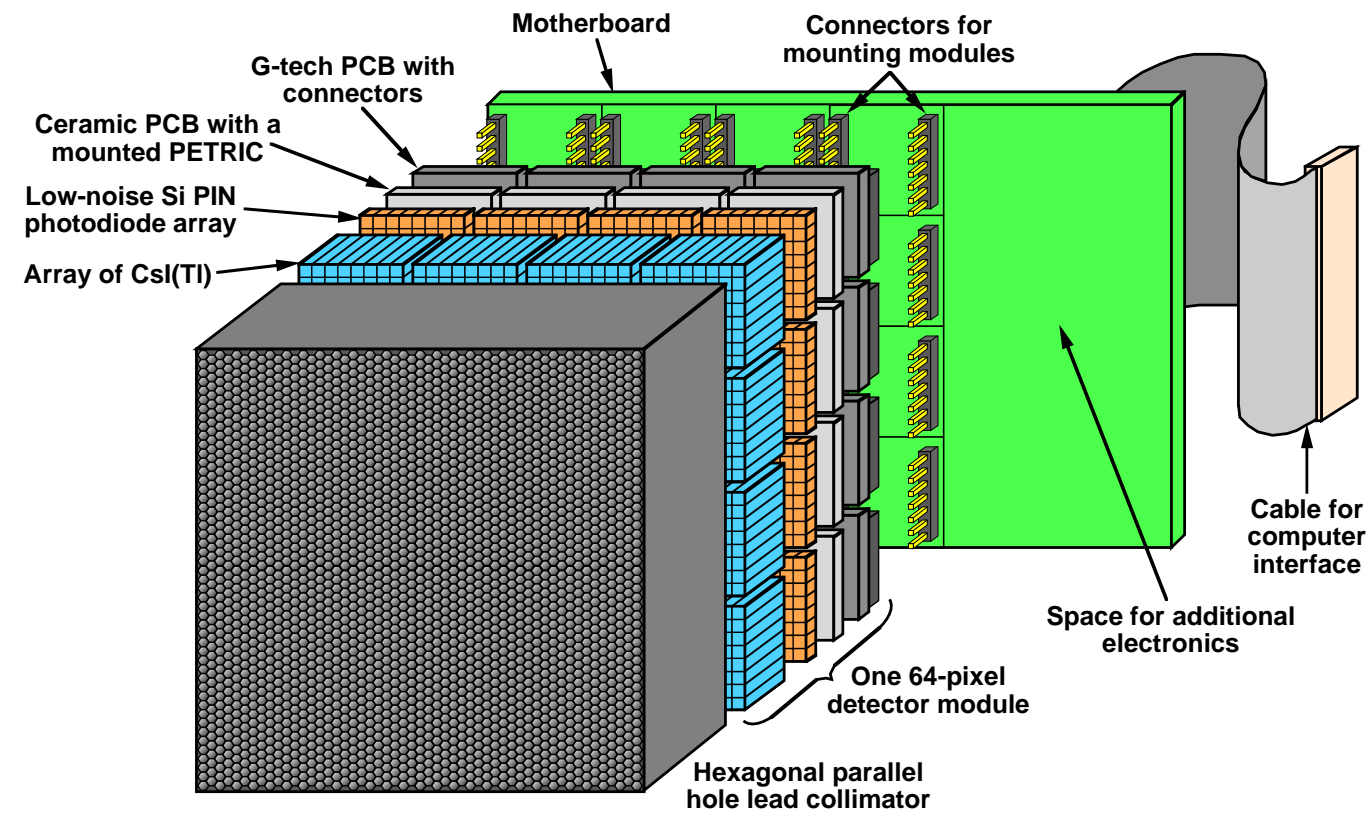

Figure 7.6: Conceptual design of a scintimammography imaging device constructed from 16 individual 64-pixel modules, forming a 1024-pixel camera. Each module plugs into a motherboard which interfaces with a computer and provides both the necessary control signals and the readout of the output signals from the individual PETRICs. While the CsI(Tl) array, photodiode array, ceramic PCB, and GETEK $\mathrm{PCB}$ are all modular, the collimator is a single piece that services the entire camera. 
to present them to the computer acquisition system in a coherent fashion. Further, it is possible to individually adjust all of the current control signals for each module individually via computer control (rather than by trimming potentiometers by hand). Communication to and from a computer is implemented by means of a general purpose analog, digital, and timing I/O board (Lab-NB, National Instruments, Austin, TX) controlled by a Macintosh system running Labview.

One of the primary concerns is the routing of the serial data and serial clock $\mathrm{I}^{2} \mathrm{C}$ communication lines to all of the modules so that the digital protocol may communicate with the PETRICs. On the motherboard itself each module is hardwired with a distinct 4-bit address so that commands on a common bus may be sent to specific modules without affecting the others. Thus single serial clock and serial data lines may service all 16 PETRICs.

Another chief concern is routing all of the output signals to the acquisition computer. At 9 outputs per PETRIC, this is non-trivial. Add on top of this the fact that each analog output needs peak detect circuitry in order to allow capture of the peak pulse height and suddenly the motherboard becomes much more complex and requires a great deal of area. One solution to ease the hardware requirements for the motherboard itself, of course, would be to simply send signals offboard to data acquisition hardware systems running in parallel: 16 separate ADCs with built-in peak detect capabilities along with 16 digital registers (for the address bits).

For my design, however, a superior solution that does not require as much expensive computer acquisition hardware is to repeat the core philosophy of the PETRIC itself- use a WTA circuit to reduce the 16 analog signals to a single winner signal plus some address bits. The 16-channel prototype WTA IC discussed in Chapter 6 is ideal for this task. The 16 individual winner signals produced by the PETRICs are routed to the WTA-only IC, which then outputs the signal with the greatest amplitude along with a 4-bit digital address denoting the module from which the winner was generated. In addition, the WTA-only IC is given control of the PETRIC enable signals (which determine whether a given PETRIC can drive its 
digital output lines or must leave them in a high-Z state, though in either case the analog output remains "on") and exploits this control by only enabling the PETRIC which is currently generating the winning signal. This then means that the 8 address plus error checking bits from each PETRIC can all be tied together, and the lines will only be driven by the PETRIC producing the winning signal. The analog output and 4-bit digital address from the WTA-only IC plus the 8 address and error checking output bits common to all PETRICs are all that need be sent to the acquisition computer. Together they comprise the analog winner signal for the entire 1024-pixel camera, the 10-bit digital address of the winning pixel, and an additional 2 bits of address error checking. These outputs can be relatively easily routed to and read out by computer acquisition hardware, which need only be a single ADC plus a 12-bit register. The WTA-only IC also sits on $\mathrm{I}^{2} \mathrm{C}$ serial clock and serial data lines and can therefore be controlled via its digital protocol.

An obvious disadvantage of employing the WTA-only IC in this manner is a decrease in the maximum event rate of the camera. Now any given event does cause camera-wide dead time because the WTA-only IC which ultimately services all pixels is temporarily occupied. However, even with this architecture the maximum event rate remains adequately high because the dead time for the 16-channel WTA IC servicing the 16 PETRICs is only about $10 \mu$ s per event (see Figure 7.8 later in this section). Assuming the worst and treating the system as fully paralyzable, Equation 3.4 indicates that a true event rate of $12 \mathrm{kHz}$, for example, would provide an observed event rate of $\sim 10.6 \mathrm{kHz}$ (a loss of less than 13\%) and would therefore generate over 6,000,000 events during a standard 10-minute study. While many of these events will be rejected on the basis of energy discrimination, the results should still be considerably more than the 100,000 s of counts that are generally utilized for a single scintimammography planar image.

One issue that has yet be addressed in detail with the WTA-only IC, however, is the peak detect circuitry necessary to properly time the analog-to-digital conversion of the overall analog winner. I have implemented on the motherboard 
a simple threshold discriminator (to eliminate low-voltage noise peaks) and a commercially available peak detect IC (PH300, Amptek, Inc., Bedford, MA). The peak detect circuitry utilizes a diode pump - a storage capacitor is charged by the analog signal through a diode, with the diode then preventing the signal from discharging the capacitor even when the analog input signal drops below its peak value. Three one-shots produce a sequence of timing pulses with carefully calibrated widths that activate the computer acquisition (both the $\mathrm{ADC}$ and the digital register trigger) and then discharge the storage capacitor through a FET once the signal pulse in question has been fully processed. This timing circuitry is implemented such that even if anything goes wrong with any of the timing pulses, the hardware will return to its default state and be prepared to process the next pulse that comes along. Thus the worst case scenario is that one particular pulse is missed, versus the possibility that the circuitry locks into a state whereby after it fails to finish processing the pulse in question, it is further unable to process any additional pulses. This circuitry is summarized in Figure 7.7.

Closely related to the design of the peak detect circuitry are timing concerns for signals in the entire readout chain-local winner signals produced by the PETRICs, the global winner signal produced by the 16-channel WTA, the address bits produced by all the readout ICs, and the signals produced by the peak detect and data acquisition systems. Figure 7.8 summarizes the timing of the different signals in the readout hierarchy and provides insight into issues such as dead time.

Among the PETRIC control signals listed in Section 7.3.3, there are 4 current signals for which it would be advantageous to have an easy means of making module-by-module adjustments: (1) preamp reset time/current compensation, (2) shaper rise time, (3) shaper fall time, and (4) WTA "dummy" channel threshold. I have implemented the ability to adjust these currents via computer control by employing E²Pot ICs (X9241, Xicor, Inc., Milpitas, CA). Each IC contains 4 nonvolatile, digital potentiometers whose resistance is adjusted electronically by means of a digital protocol. Thus a single $\mathrm{E}^{2}$ Pot IC is sufficient to provide easy adjustment of 


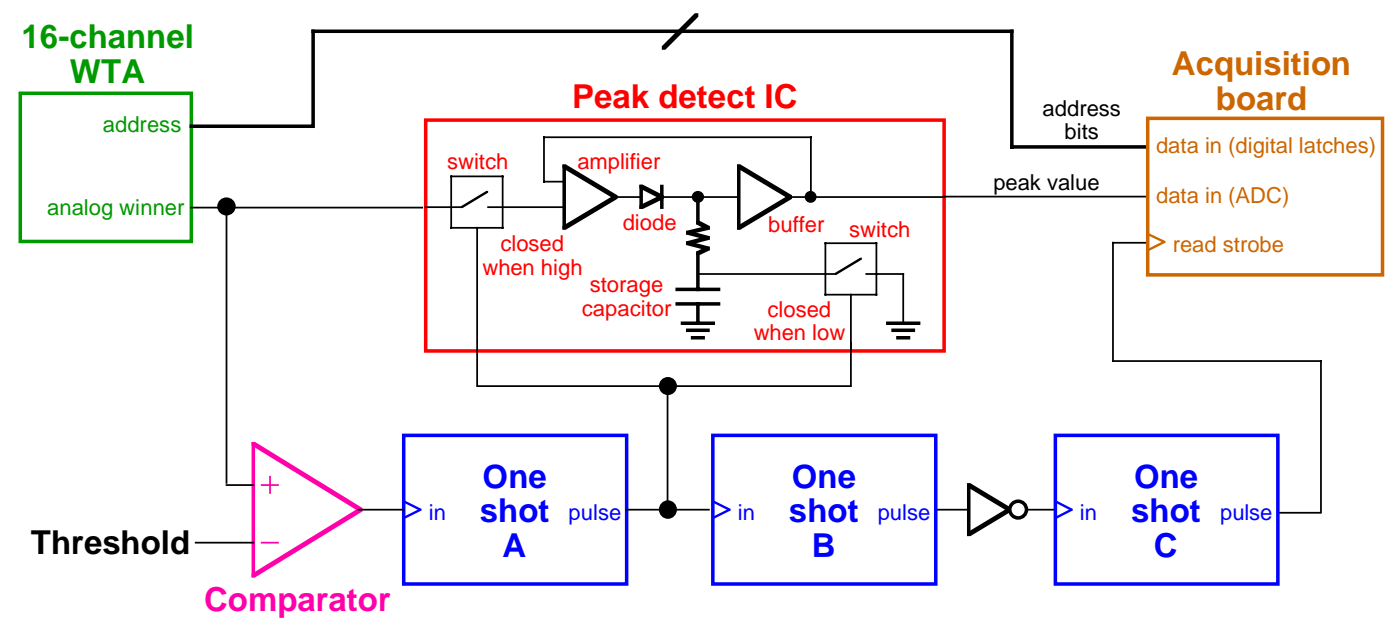

Figure 7.7: Peak detect circuitry employed on the 16-module motherboard. When the analog winner signal exceeds the voltage threshold, the comparator output will go high, activating one shot $\mathrm{A}$. The output pulse from one shot $\mathrm{A}$ in turn activates one shot B, which also generates an output pulse. The output from A also activates the peak detect IC - so long as the pulse from A is high, the IC will track the analog winner to its peak value and hold it. When the pulse from A expires and returns to ground, the IC will discharge its hold capacitor and return to a resting state. One shot $\mathrm{B}$, whose pulse duration must be set to less than that of $\mathrm{A}$, merely serves as a delay element. Once the pulse from B expires, the inverter on its output produces a positive edge which activates one shot $\mathrm{C}$. The output pulse from $\mathrm{C}$ is very short in duration and acts as a read strobe which triggers data collection by the acquisition board. The pulse from A should still be active at the time of data acquisition. Note that the one shots all ignore any input triggers while their output pulses are high, providing some protection against the readout chain being interrupted. Not shown are the address bits from the PETRICs that the 16-channel WTA services-these bits are collected at the same time as the WTA address bits. A timing diagram for the signals relevant to this system is presented in Figure 7.8. 


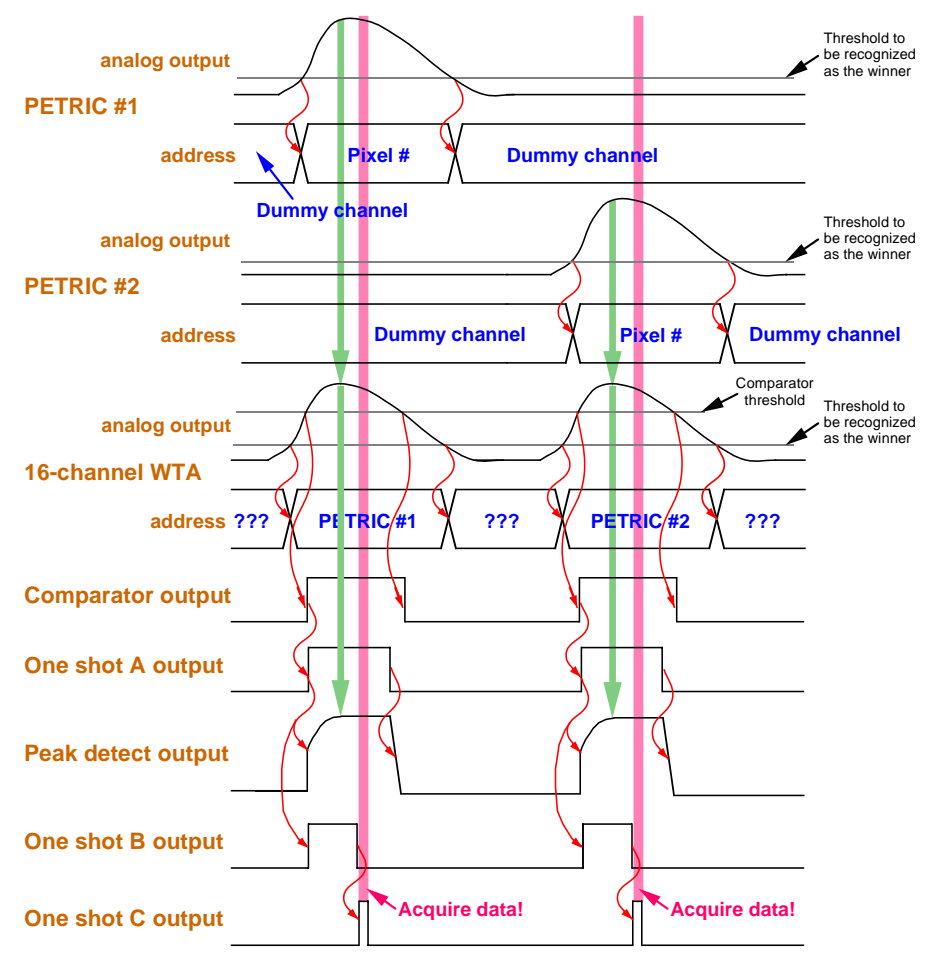

Figure 7.8: Timing diagram for important data acquisition signals in the 16-module camera design. A simple case involving two PETRICs being serviced by the 16channel WTA is presented. Light green arrows indicate analog waveforms that are replicated in whole or in part on another line. Blue text indicates the value of digital address bits. Red arrows indicate causality amongst the signals (for the actual wiring see Figure 7.7). The light pink lines indicate the window during which the computer acquires the peak detect output voltage, the 16-channel WTA address bits, and the address bits from the PETRIC that is currently generating the winning signal. Acquisition must occur after the analog pulse has reached its peak value but before either: (1) the peak detect returns to a resting state or (2) the address bits generated by the 16-channel WTA and relevant PETRIC are no longer valid. The chain of events required to successfully collect data for one gamma essentially begins when the comparator fires and ends when both the comparator and the peak detect IC have returned to their resting states. This then determines the camera dead time, which for an $8 \mu$ s peaking time can be made to be about $10 \mu \mathrm{s}$. 
all 4 currents for a given module. The $16 \mathrm{E}^{2}$ Pot ICs servicing the 16 modules receive commands via the $\mathrm{I}^{2} \mathrm{C}$ protocol as do the PETRICs, and likewise the 16 different ICs are distinguished from each other by hardwired 4-bit addresses. Separate serial clock lines are used for the PETRICs and for the $\mathrm{E}^{2}$ Pots, however, to prevent commands on the serial data line that are intended for one type of chip from being latched into the chip of the other type with the same 4-bit address.

My final motherboard design is summarized in the block diagram in Figure 7.9. Although they are not included in the diagram, a substantial number of level conversions between TTL and ECL logic levels are also implemented to ensure that the various components can all communicate properly. The final layout for the motherboard calls for an 8-layer FR-4 board with a substantial thickness of 0.093 inches in order to give it mechanical strength and rigidity. Care has been taken to minimize crosstalk, especially from the large analog output pulses and the digital lines back to the sensitive analog inputs of the PETRICs. This includes not so much crosstalk from the motherboard directly to the inputs directly (since the distances involved are great enough to minimize this problem), but rather noise injection into sensitive lines critical to the input signals, namely the $50 \mathrm{~V}$ photodiode backside bias and the $-1.65 \mathrm{~V}$ photodiode guard ring bias. Care has also been taken to make the power and ground planes as stable as possible in order to maintain a low-noise environment for the PETRICs.

The 16-module motherboard has been successfully fabricated and the necessary electrical components loaded onto it. The board is currently undergoing debugging, and while the it is not yet operational, no insurmountable problems have been uncovered.

\subsubsection{Collimator Design}

Based on the simulation results from Chapter 5, I have designed and acquired three collimators for use with the 16-module camera. All three collimators are 


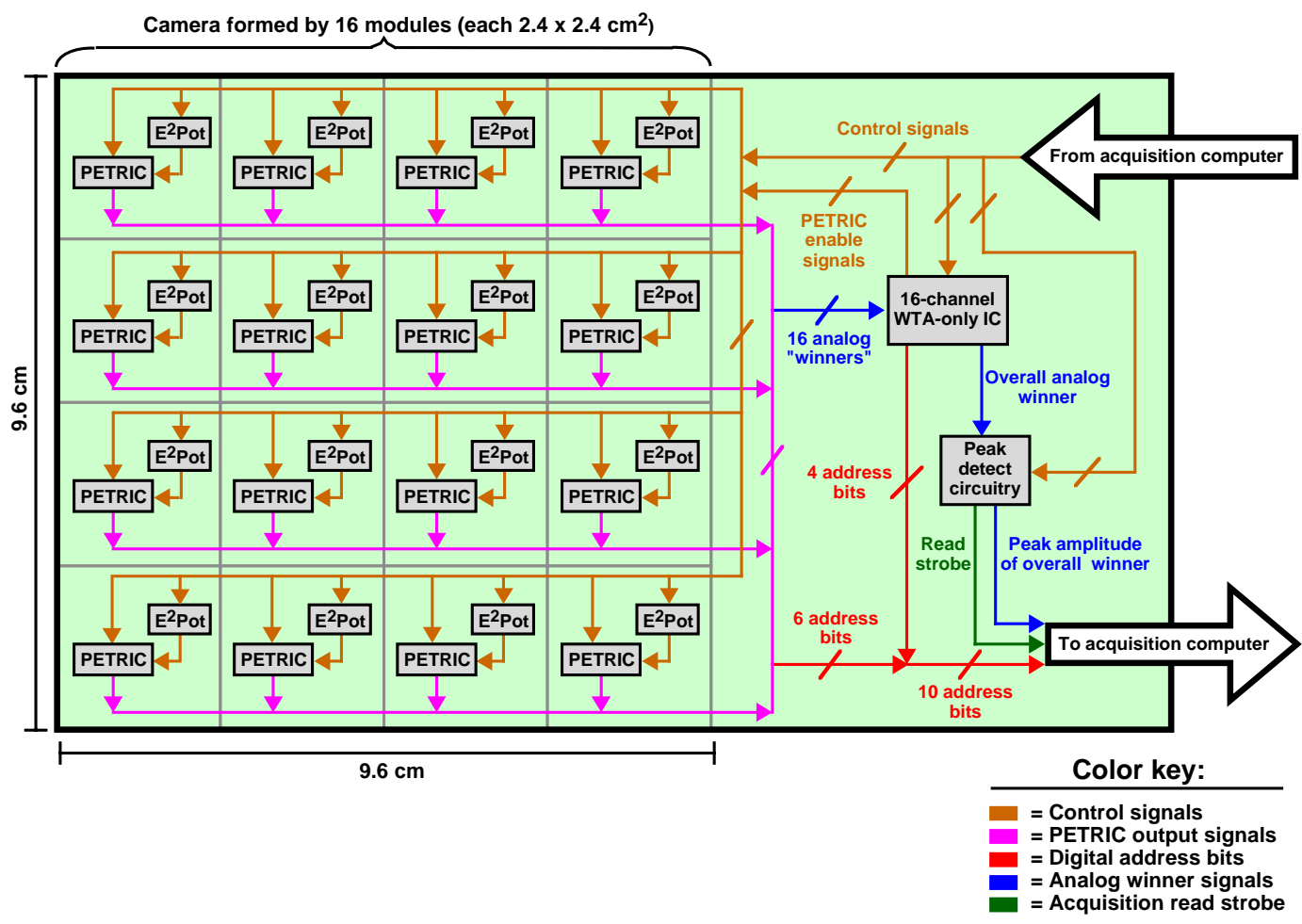

Figure 7.9: Block diagram of the 16-module motherboard used to integrate 16 64pixel modules into a 1024-pixel camera. The motherboard routes control signals from the acquisition computer to the individual ICs, the output analog "winner" signals from the PETRICs to a WTA-only IC, and output address bits from both the PETRICs and the WTA-only IC to the acquisition computer. The overall analog "winner" for the entire camera is routed from the WTA-only IC to peak detect circuitry, and then ultimately to the acquisition computer. 
microcast from lead, cover an area of $10 \times 10 \mathrm{~cm}^{2}$, and employ parallel hexagonal holes with a diameter of $1.5 \mathrm{~mm}$ and a septal thickness of $0.25 \mathrm{~mm}$. The only difference between the collimators is the channel length, which determines the sensitivity and spatial resolution. Ideally the collimators would have smaller holes $1.0 \mathrm{~mm}$ in diameter, as suggested by the Monte Carlo studies, but the manufacturer (Nuclear Fields, Inc., Des Plaines, IL) cannot achieve that without expensive custom tooling. The $1.5 \mathrm{~mm}$ holes are less ideal but nonetheless acceptable since the pixels are still twice as large at $3 \mathrm{~mm}$ pitch (Equation 4.15). As has been mentioned, future collimator designs may potentially utilize square hole tungsten laminate designs, but the gains to be had are reasonably small and the expense substantially higher.

Table 7.1 provides the design specifications for the three collimators. As per the simulation results, the collimators have been designed with high sensitivities (ranging from 8000 to 26,000 counts $/ \mathrm{mCi} / \mathrm{sec}$ ) in order to improve the $\mathrm{S} / \mathrm{N}$ of any high-uptake objects in the resulting planar images. The spatial resolutions of $6.1-$ $9.8 \mathrm{~mm}$ fwhm at $6 \mathrm{~cm}$ imaging distance still provide for the possibility of detecting tumors less than $1 \mathrm{~cm}$ in diameter, especially if the $\mathrm{S} / \mathrm{N}$ in the planar image is reasonably high. The "high sensitivity" collimator design was chosen as the intermediate configuration because it is an excellent match for the simulated collimator design in Chapter 5 with the best performance.

\subsection{Single 64-Pixel Module Performance}

In this section I describe basic performance measurements made on a complete 64-pixel module assembled according to the design and procedures set forth in Section 7.3. The module is in all ways operational and data were collected via an acquisition computer that issued control signals to the module and collected the data it generated. The 16-module motherboard which is designed to multiplex multiple modules in the final camera architecture (Section 7.4.1) was not employed because it is currently undergoing final debugging. The absence of the motherboard in this 


\begin{tabular}{|c|c|c|c|}
\cline { 2 - 4 } \multicolumn{1}{c|}{} & $\begin{array}{c}\text { All } \\
\text { purpose }\end{array}$ & $\begin{array}{c}\text { High } \\
\text { sensitivity }\end{array}$ & $\begin{array}{c}\text { Ultra high } \\
\text { sensitivity }\end{array}$ \\
\hline Channel length $(\mathrm{mm})$ & 23.5 & 16.5 & 13.0 \\
Sensitivity (counts/mCi/sec) & 8020 & 16,200 & 26,200 \\
Spatial resolution @ 6 cm (mm) & 6.1 & 8.0 & 9.8 \\
Septal penetration (\%) & 0.6 & 2.6 & 5.6 \\
\hline
\end{tabular}

Table 7.1: Three collimator designs for use with the 16-module camera. The designs all provide relatively high sensitivity in order to provide good $\mathrm{S} / \mathrm{N}$ for small tumors imaged in the photon-starved scintimammography environment. In all cases the septal thickness is $0.25 \mathrm{~mm}$.

experimental setup also removes the peak detect circuitry from the readout chain, however, and therefore it was necessary to implement an alternate peak detect system. This took the form of a CAMAC ADC with built-in peak detect capability. However, because the histogramming memory associated with this ADC can store only one histogram at a time, this setup precludes imaging with multiple pixels simultaneously. As such, the computer control of the PETRIC in the module was programmed to scan through channels sequentially, generating an effective 64-pixel image (but requiring 64 times as much imaging time). While the PETRIC pixel identification bits were therefore not needed, the accuracy of this output vector was verified by supplying a test pulse to individual channels and observing the bit pattern. Finally, the computer control of the PETRIC was also programmed to set the input current compensation for each channel to the lowest value that would reliability keep the channel out of saturation, thereby minimizing electronic noise.

The module inputs (which supply the PETRIC with control signals) were connected to a series of computer-controlled DACs and the module output connected to the peak-detecting CAMAC ADC by employing a simple circuit board. The board is a temporary replacement for the final motherboard and provides the necessary signal routing for a single module, as well as offering the same connector geometry 
as the motherboard so that a 64-pixel module may simply plug into it.

Once the 16-module motherboard (and the peak detect circuitry it houses) is operational and is employed during future measurements, scanning through pixels one at a time will be unnecessary. Instead, a simpler ADC (without the need to offer peak detect capability) will be used to digitize pulse height values from the output of the motherboard peak detect circuitry. These pulse height voltages will then be acquired by the computer synchronously with the PETRIC address bits and written to separate histogram data files based on the pixel identified. Thus, 64 histograms will be simultaneously generated.

The 64-pixel module whose performance is studied herein is completely ready to be plugged into the 16-module motherboard once that hardware is available. The module itself, even with the pixel scanning readout, therefore provides an excellent albeit preliminary means of characterizing the basic performance of this camera technology. Further, because the module is a relatively early version of the 64-pixel devices, performance improvements are to be expected in the future as the assembly techniques are refined.

It was noted from the onset that 4 of the 64 pixels is this particular module are completely non-responsive because those channels of the readout IC are dead. Whether this is due to processing errors during the fabrication of the PETRIC or due to damage during the process of mounting the chip onto the circuit boards is unclear. Since these pixels are near the periphery of the module (see Figure 7.11), however, this is a relatively minor problem that does not impede the basic measurements presented below. Further, since other circuit board assemblies with mounted PETRICs have successfully demonstrated 64 healthy and operational readout channels, this is not expected to be an ongoing problem. 


\subsubsection{Intrinsic Energy Resolution}

I measured the intrinsic energy resolution of the 64-pixel module by exciting it with $122 \mathrm{keV}$ gammas from a $100 \mu \mathrm{Ci}{ }^{57} \mathrm{Co}$ source in air. No collimator was employed during the measurement, the entire experimental assembly was covered with a large black cloth to ensure darkness, the radioactive source was kept $15 \mathrm{~cm}$ from the front face of the $\mathrm{CsI}(\mathrm{Tl})$ array, and the imaging time was 10 minutes per pixel. A thin layer of grounded aluminum foil was wrapped around the module to improve EM shielding.

Photopeak spectra for 8 typical pixels in the module are displayed in Figure 7.10. Pulse heights are presented in terms of histogram bin number because signal levels were not calibrated across the entire 64 channels. Calibration measurements were performed on a few channels prior to attaching the $\mathrm{CsI}(\mathrm{Tl})$ array, however,

by measuring the pulse height of $5.9 \mathrm{keV}$ gammas from an ${ }^{55} \mathrm{Fe}$ source interacting directly in the silicon photodiodes, as this is known to generate an average signal of $1645 \mathrm{e}^{-}$. Results from these calibration measurements suggest an average $122 \mathrm{keV}$ photopeak signal level of $3500 \mathrm{e}^{-}$. Energy resolution was measured by selecting only the histogram bins required to cover both the escape peak and the photopeak for a given channel, simultaneously fitting two Gaussians to these overlapping peaks, and then calculating the fwhm of the photopeak Gaussian. The average energy resolution across the operational channels is $20.0 \pm 2.6 \%$ fwhm. Assuming a linear response with respect to gamma energy, $140 \mathrm{keV}$ gammas from ${ }^{99 m} \mathrm{Tc}$ have an expected signal level of about $4000 \mathrm{e}^{-}$and will yield an energy resolution of about $17.4 \pm 2.3 \%$ fwhm.

\subsubsection{Uniformity of Response to a Flood Source}

Using the same data set collected for energy resolution calculations in Section 7.5.1, I assembled a flood source image for the 64-pixel module. Because the $100 \mu \mathrm{Ci}, 5 \mathrm{~mm}$ diameter ${ }^{57} \mathrm{Co}$ source was imaged at a distance of $15 \mathrm{~cm}$ without a collimator present, there was little difference in the solid angle presented by differ- 


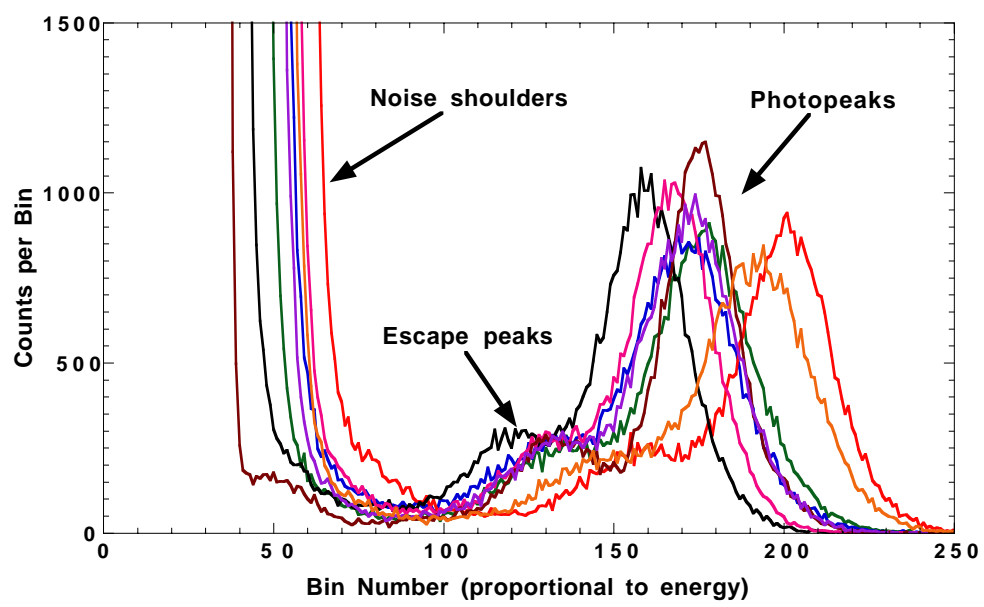

Figure 7.10: Photopeak spectra for 8 typical channels in a 64-pixel module excited by $122 \mathrm{keV}$ gamma rays from a ${ }^{57} \mathrm{Co}$ source in air. Average intrinsic energy resolution for the module is $20.0 \pm 2.6 \% \mathrm{fwhm}$, and the average photopeak signal level is about $3500 \mathrm{e}^{-}$. For $140 \mathrm{keV}$ gammas from ${ }^{99 m} \mathrm{Tc}$ the expected signal level would therefore be about $4000 \mathrm{e}^{-}$and the energy resolution about $17.4 \pm 2.3 \%$ fwhm.

ent pixels. The activity therefore was a reasonable representation of a flood source. The number of counts in each pixel was determined by summing the total number of events in both the escape peak and the photopeak for each spectrum. Because of the substantial overlap and blurring between the escape peak and photopeak in some channels with poor energy resolution, this method of counting produced more reliable and repeatable results than did restricting the acceptance to a more standard window defined by: photopeak signal level \pm fwhm energy resolution. Further, because no scattering medium was present, a narrow acceptance window was not required to reject scattered photons. A normalized presentation of the flood source response is displayed in Figure 7.11. The average number of counts detected per pixel (excluding the 4 dead pixels) is $41,400 \pm 1100$. While visually the response looks fairly uniform, the standard deviation of 1100 is almost 5.5 times the statistical noise of sqrt41, $400 \approx 203$. It is therefore relatively poor compared to modern scintillation 


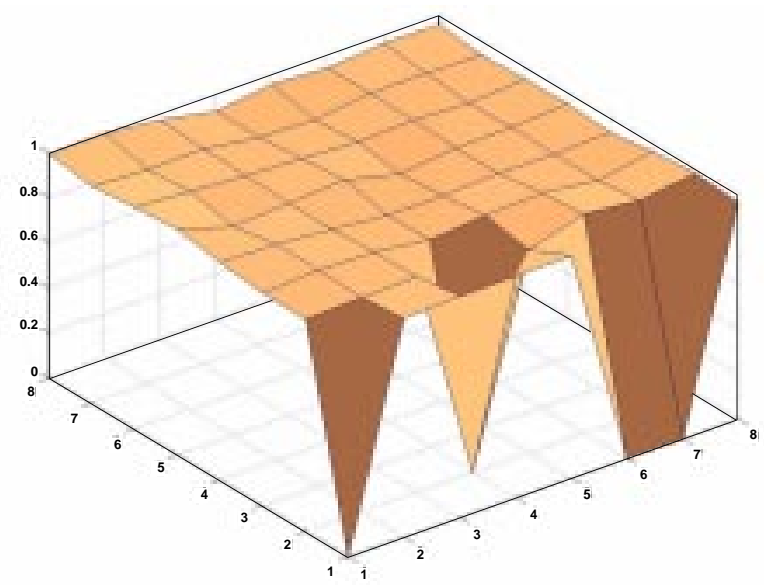

Figure 7.11: Normalized response of the pixels in a 64 -channel module to a ${ }^{57} \mathrm{Co}$ flood source in air over a period of 10 minutes. Four dead pixels are readily identifiable. The average counts per pixel is $41,400 \pm 1100$.

cameras, which generally achieve a uniformity standard deviation of less than 3 times the statistical noise. Future 64-pixel modules will naturally seek to improve upon this - better energy resolution, for example, should make it easier to count the total number of events detected in each channel in a more accurate manner, contributing to improved flood source images.

\subsubsection{Point Source Images}

I implemented a "point" source by placing the $5 \mathrm{~mm}$ diameter, $100 \mu \mathrm{Ci}$

${ }^{57} \mathrm{Co}$ source behind a lead sheet with a $2.7 \mathrm{~mm}$ diameter hole in it. Images were then generated using the 64-pixel module equipped with a high sensitivity collimator (see Table 7.1 for specifications). The point source was imaged for 10 minutes at a distance of $5 \mathrm{~cm}$ both in air and behind $5 \mathrm{~cm}$ of water serving as a scattering medium. The number of counts for each pixel was calculated using a standard energy acceptance window: average photopeak pulse height \pm fwhm energy resolution, where the pulse height and energy resolution for each channel were previously calculated in 
Section 7.5.1. The resulting point source images are displayed in grayscale format in Figure 7.12. The 4 dead pixels have been smoothed out by averaging the values of adjacent pixels, but in any case the deficient pixels are not in critical locations and contribute extremely little information to the images.

Spatial fwhm values were calculated for the point images by fitting a Gaussian in both the $\mathrm{x}$ and $\mathrm{y}$ directions along the row with the most counts, then determining the fwhm value of that Gaussian. The point source imaged in air is $7.1 \mathrm{~mm}$ fwhm in the $\mathrm{x}$ direction and $8.0 \mathrm{~mm}$ fwhm in the y direction, or an average of $7.6 \mathrm{~mm}$ fwhm in size. For the point source imaged with the water scattering medium, the results are $7.4 \mathrm{~mm}$ fwhm in $\mathrm{x}$ and $8.1 \mathrm{~mm}$ fwhm in $\mathrm{y}$ for an average of $7.8 \mathrm{~mm}$ fwhm. Inspecting the images in Figure 7.12 visually does suggest that the point image with water is slightly more diffuse. Certainly it is reasonable to expect that scatter produced in the water would adversely affect the detector spatial resolution, which is seems to do to a small degree. Since the spatial resolution of the high sensitivity collimator is about $6.8 \mathrm{~mm}$ fwhm at $5 \mathrm{~cm}$ imaging distance, the point source is really $2.7 \mathrm{~mm}$ in diameter, and the pixel pitch is $3 \mathrm{~mm}$, these three values add in quadrature to give a first order theoretical spatial resolution of about $7.9 \mathrm{~mm}$ fwhm. Obviously this is consistent with the observed results.

Finally, the peak number of counts in a pixel for the point source in air is about 43,400, compared to about 19,900 for the point source behind $5 \mathrm{~cm}$ of water. The addition of water therefore represents a decrease in signal to about $46 \%$ of the without water scenario, consistent with the attenuation of $122 \mathrm{keV}$ gammas through $5 \mathrm{~cm}$ of water (attenuation length $\approx 65 \mathrm{~mm}$ ). The minimum number of counts observed in any operational pixel in both setups was approximately 40, providing a max-to-min ratio of about 1100 in air and about 500 with the water. Certainly when imaging a point source in the absence of any background radiation an extremely high contrast is to be expected. 


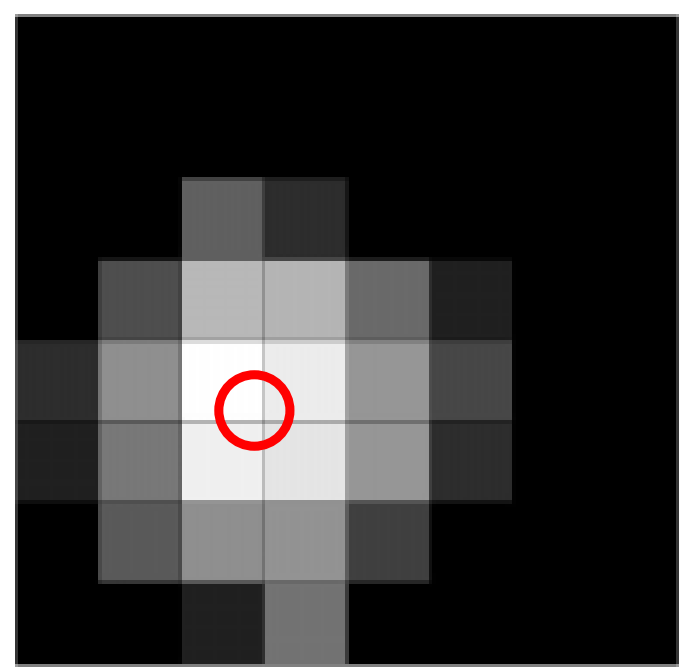

(a)

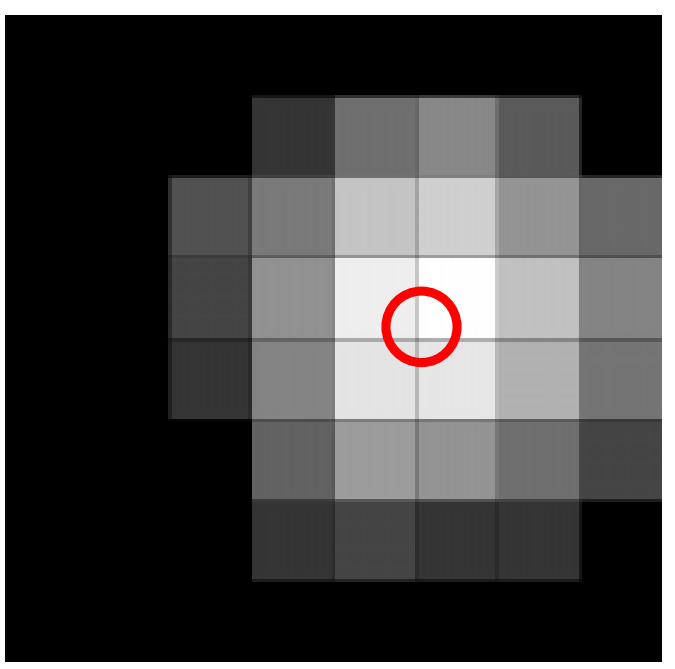

(b)

Figure 7.12: Grayscale images of a ${ }^{57}$ Co point source (formed by placing the $5 \mathrm{~mm}$ diameter, $100 \mu \mathrm{Ci}$ source behind a lead sheet with a $2.7 \mathrm{~mm}$ diameter hole in it) imaged by a 64-pixel module at an imaging distance of $5 \mathrm{~mm}$ for 10 minutes. A high sensitivity collimator (see Table 7.1 for specifications) was employed in front of the detector module. The red circles represent the approximate size and location of the source. The image in (a) represents the point source in air, while that in (b) represents the point source behind $5 \mathrm{~cm}$ of water. The average spatial fwhm value for the image in (a) is $7.6 \mathrm{~mm}$, while that for the image in (b) is $7.8 \mathrm{~mm}$. The grayscale for both images is set such that the peak pixels are white and pixels with $2.5 \%$ of the peak pixel counts are barely recognizable as non-black. The peak number of counts in (b), however, is only $46 \%$ of that in (a) because of attenuation in the water. The 4 dead pixels are near the tops of the images and have been smoothed out. 


\subsubsection{Module Performance Summary}

As is indicated in Section 7.5.1, the 64-pixel module demonstrates average energy resolution of $20.0 \pm 2.6 \%$ fwhm for $122 \mathrm{keV}$ gammas, which suggests a resolution of $17.4 \pm 2.3 \%$ fwhm for $140 \mathrm{keV}$ gammas. While this result is adequate to make the photopeaks and escape peaks distinct, it is nonetheless disappointing. The 12-pixel prototype module characterized in Section 4.3.2, for instance, demonstrates a much smaller energy resolution of only $10.7 \pm 0.6 \%$ fwhm. Given that in both cases the readout ICs exhibit similar noise behavior and the photodiodes have similar load properties, this is somewhat surprising. However, with the 12-pixel module the $140 \mathrm{keV}$ photopeak signal levels are about $6600 \mathrm{e}^{-}$, compared to a mere $4000 \mathrm{e}^{-}$ or so for the 64-pixel module. An increase in the 64-pixel module signal levels to $6600 \mathrm{e}^{-}$for $140 \mathrm{keV}$ gammas would drop the associated energy resolution to about $10.5 \%$ fwhm, obviously consistent with the 12-pixel module results. The cause of this loss of signal is not immediately clear, though given that the quantum efficiency of the Si PIN photodiodes has been measured at over $80 \%$ for $540 \mathrm{~nm}$ scintillation photons, the most likely culprit would seem to be the $\mathrm{CsI}(\mathrm{Tl})$ itself. Low light output crystals (either due to low quality $\mathrm{CsI}(\mathrm{Tl})$ or defective reflecting material) or errors in aligning and epoxying the $\mathrm{CsI}(\mathrm{Tl})$ array to the photodiode array could account for the signal loss. In any case, future development and construction of 64-pixel modules will focus on exploring this issue.

The Monte Carlo simulations in Chapter 5, however, suggest that improving energy resolution much below $15 \%$ fwhm for $140 \mathrm{keV}$ gammas is of marginal value for scintimammography geometries where scatter is minimized (such as the craniocaudal view). Thus the average result of $17.4 \%$ fwhm may not be particularly high or problematic. Coupling this with the likelihood of improving signal levels and energy resolution in the future, these results can be considered encouraging even if imperfect. In order to use this technology with a wider range of imaging geometries which may include significant scatter, however, achieving better energy resolution remains very 
desirable.

Preliminary characterizations of the 64-pixel module imaging capabilities in Sections 7.5.2 and 7.5.3 are also encouraging, if imperfect. The flood source image without a collimator indicates a reasonably uniform response (with the exception of the dead pixels), but one that is still lacking compared to modern scintillation cameras. The two images of a point source in air and with water as a scattering medium demonstrate the expected spatial fwhm values and clearly indicate the location of the source. Thus the ability of the 64-pixel modules to image simple activity distributions has been demonstrated. Another imaging characterization it would be useful to perform would be to image a true, distributed flood source with a detector module employing a collimator. This would show how uniform the response of the imaging technology is with the collimator hexagonal hole-square detector pixel aliasing present. Further, it would allow for the calculation of individual pixel sensitivity correction factors that could be used to improve the pixel data in other acquisitions and improve image quality (e.g., Figure 7.12).

Thus the performance of the 64-pixel module suggests that the advantages $\mathrm{CsI}(\mathrm{Tl}) / \mathrm{Si}$ PIN photodiode scintillation cameras theoretically enjoy over traditional Anger cameras (Chapter 3) can be realized. With further development such cameras may well be worthy of clinical trials designed to quantify their ability to detect and diagnose breast cancer in the roles I outlined in Chapter 2. Future directions and camera characterizations that need be pursued to make this a reality are discussed in Chapter 8. 


\section{Chapter 8}

\section{Conclusion}

Each problem that I solved became a rule which served afterwards to solve other problems.

During the course of thesis project numerous contributions have been made to the development of compact gamma cameras for breast cancer imaging. These advances include detector development, readout electronics design, simulation studies of scintimammography imaging, the design and construction of compact 64-pixel modules, and the design and preliminary development of a 16-module camera. At all times this technology has been developed specifically for real world breast cancer applications, and the success of this effort is demonstrated by the fact that two separate companies are currently developing commercial scintimammography cameras based in part on the hardware, readout design, and Monte Carlo studies described in this thesis. This project, of course, remains a work in progress, for the accomplishments to date raise new research questions and open significant new possibilities for future compact gamma camera development. This chapter summarizes the key innovations that have been made and discusses future directions for the continuation of this work. 


\subsection{Summary of Results}

The first step in developing this new technology was the identification of the specific scintimammography tasks compact gamma cameras can potentially perform, thereby defining many of the instrumentation design goals. After exploring the available literature covering both breast cancer screening and clinical trials employing a wide range of imaging and diagnostic techniques, I codified this information into a decision-making tree summarizing the procedures used to detect, diagnose, and treat breast cancer. Key possibilities for compact, discrete single photon cameras include evaluating women with radiographically dense breasts, evaluating axillary lymph node involvement, checking for multicentricity, measuring tumor response to chemotherapy as treatment progresses, and discriminating post-surgery tumor recurrence from scar tissue. Such cameras may also aid in the evaluation of the efficacy of new radiopharmaceutical agents.

The key and innovative achievement in the area of detector design is the development of 64-pixel low-noise Si PIN photodiode arrays. These are the first photodiodes with both sufficient reliability and low enough leakage current (i.e., low enough electronic noise) to make $\mathrm{CsI}(\mathrm{Tl}) /$ photodiode scintimammography cameras with acceptable energy resolution possible. Development efforts have included not only the initial design and the gettering procedures which greatly limit ion contamination of the silicon, but also the scaling up from 12 to 64 pixels and the improvement in the photodiode yield to an acceptable level. Further, the proper means of making electrical connections to the photodiode arrays and of packaging them during the assembly procedures were established. It was shown that these arrays: (1) offer excellent noise properties because the photodiode leakage current at $50 \mathrm{~V}$ bias averages only $26 \mathrm{pA}$ for $3 \times 3 \mathrm{~mm}^{2}$ pixels, (2) have high quantum efficiency greater than $80 \%$ for the $540 \mathrm{~nm}$ scintillation light from $\mathrm{CsI}(\mathrm{Tl})$, (3) can be manufactured with a reasonably high pixel yield of $98.5 \%$, and (4) can be efficiently incorporated into

compact imaging modules designed specifically for scintimammography. The end 
result is that the PMTs used in Anger cameras may be replaced in favor of smaller and cheaper photodetectors. My specific contributions to this photodiode research include developing specifications for the photodiodes, working with commercial companies to develop non-destructive dicing techniques, developing the assembly and packaging procedures that integrate these delicate arrays into the imaging modules, and finally, measuring and analyzing: (1) leakage current, (2) quantum efficiency, (3) signal levels, and (4) noise performance.

The major achievement in the area of electronics readout is the development, debugging, and characterization of the 64-channel PETRIC, leading to its successful integration into imaging modules. At the core of this CMOS ASIC are low-noise amplifiers and the unique WTA circuit which greatly reduces the number of output lines. Both have proven advantageous in the design and implementation of scintimammography instrumentation. Further, the PETRIC offers a great deal of flexibility via external control signals and a comprehensive digital protocol, making modification of the chip's behavior with a computer straightforward. It was established that: (1) computer control of the PETRIC is fully functional and the chip is therefore very flexible, (2) the chip provides sufficiently low-noise performance (at $8 \mu$ s peaking time the electronic noise is as low as $180 \mathrm{e}^{-}$rms with a custom $\mathrm{Si}$ PIN photodiode load), (3) the PETRIC correctly identifies the "winner" with its 6 address bits in less than $150 \mathrm{~ns}$, and (4) integrating multiple ICs into a multi-module readout architecture for an entire camera is effective and compact. Taken as a whole, these results demonstrate that an ASIC readout architecture can service a complete $\mathrm{CsI}(\mathrm{Tl}) /$ photodiode scintimammography camera, whereas discrete component readout systems are infeasible for 100s or 1000s of pixels.

The PETRIC development project has obviously involved a great deal of effort by numerous people. My specific contributions have included developing specifications for the IC, characterizing and debugging multiple prototype front end ICs, characterizing and debugging a preliminary readout system formed by using both a front end and a WTA prototype IC, both physically and electrically integrating the 
PETRIC into the severely space-constrained imaging modules, and characterizing PETRIC performance when integrated into a complete imaging module.

I have explored the specific challenges of imaging tracer-avid tumors in the breast with a compact, discrete gamma camera by developing and executing a series of Monte Carlo simulations. I wrote the necessary code and conducted these simulations with the goal of contributing to design decisions for cameras optimized for scintimammography. The code was therefore designed to provide a realistic representation of actual clinical situations, including a 3-D phantom patient, background events from healthy tissue, photoelectric absorption, and Compton scatter. The program is also flexible and offers the ability to easily change tumor size, tumorto-background uptake ratio, tumor position, collimator configuration, detector pixel size, detector energy resolution, and even phantom patient geometry. Simulated planar images suggest that in some cases it may be possible to observe tumors smaller than $1 \mathrm{~cm}$ in diameter, but also highlight the difficulties in this endeavor, especially when tumors are smaller than $0.75 \mathrm{~cm}$. Results also demonstrate: (1) the importance of using a high sensitivity collimator $(>16,000$ counts $/ \mathrm{mCi} / \mathrm{sec})$ in the event-starved scintimammography application, (2) the diminishing value of making detector pixels much smaller than $3 \mathrm{~mm}$ pitch, (3) the rather minor advantages of replacing standard hexagonal collimator holes with square holes matched to the detector pixels, (4) the relative unimportance of improving energy resolution much beyond $15 \%$ fwhm (e.g. by cooling the detectors) for craniocaudal scintimammography imaging, (5) the important influence of tumor tracer uptake relative to uptake by healthy tissue. The results of the simulation study suggest that for scintimammography it is entirely reasonable to employ a high sensitivity hexagonal hole collimator with $3 \mathrm{~mm}$-pitch detectors operated at room temperature. While it is possible to improve certain performance metrics with a more aggressive (and hence more costly and more difficult to implement) design, the simulation study suggests that the benefits may be marginal for scintimammography applications.

A final accomplishment to pull the aforementioned innovations together is 
the design and preliminary development of a complete camera potentially suitable for scintimammography studies. I have contributed to this effort by developing and characterizing prototype proof-of-concept 12-pixel CsI(Tl) scintillator/Si PIN photodiode/ASIC detector modules, designing custom printed circuit boards for use with the 64-pixel photodiode arrays and the PETRIC, developing procedures to reliably assemble 64-pixel modules, specifying and designing the motherboard which interfaces with 16 modules and an acquisition computer, and finally, assembling, testing, and characterizing the final 64-pixel imaging modules. The implementation emphasizes maximally compact design and incorporates the knowledge gained from the Monte Carlo simulation studies. A prototype 64-pixel module demonstrates average energy resolution of $20.0 \pm 2.6 \%$ fwhm for $122 \mathrm{keV}$ gammas from ${ }^{57} \mathrm{Co}$, or an estimated $17.4 \pm 2.3 \%$ fwhm for $140 \mathrm{keV}$ gammas from ${ }^{99 m} \mathrm{Tc}$. A flood source irradiating the detector array without a collimator produces a reasonbly uniform image with a standard deviation of $2.7 \%$ of the average count rate. Finally, a point source (in air or with scattering media) exciting a module equipped with a collimator produces images with the anticipated collimator-limited spatial resolution.

Based on the compact size of the camera technology as well as these results, clearly the first steps have been made toward producing a clinical scintimammography camera. With further refinement and development, the design presented in this thesis may well be appropriate for this task.

\subsection{Future Work}

Additional development and testing is clearly necessary before this new scintimammography technology is complete and ready for clinical trials. The primary module components - the photodiode arrays, PETRIC, and module PCBs - are all operational with few flaws, and the 16-module motherboard is fabricated and looks promising as it undergoes debugging. A prototype complete 64-pixel module has thus far proven successful, though further effort is necessary to improve energy resolution 
in future modules. Nonetheless, significantly more testing, characterization, and development of the modules as well as the camera is clearly necessary. Following that, the first step is to perform comprehensive measurements to characterize the technology in full accordance with NEMA standards. It would then be instructive to perform ROC studies with phantom breasts/tumors and expert observers. This would offer insight into true tumor detectability under various conditions and may justify breast cancer clinical trials. Finally, clinical trials themselves would seek to determine the sensitivity and specificity of the new camera for breast cancer compared to both Anger cameras and traditional X-ray mammography, leading to improved understanding of what clinical roles this technology is suitable for.

The instrumentation itself can certainly be further improved as well. The PETRIC, for instance, has proven fundamentally sound but can be refined. The peak detect circuitry should be incorporated into the IC itself to lessen the signal processing requirements of the motherboard and coupling between certain signals within the IC should probably be lessened. While the PETRIC currently demonstrates adequate noise performance, future versions will strive to improve this, if possible. An excellent starting point for this would be careful noise studies and comparisons of the PETRICs that have already been fabricated. While this thesis has focused entirely on the most promising PETRIC design, a total of 4 versions with slightly different biasing and power supply layouts have been fabricated and are available to offer insights on how to further optimize the IC. Another valuable approach to improving the PETRIC would be to decrease coupling from the digital output bits to the analog output signal when these digital lines are enabled. Finally, although input protection diodes have been implemented and studied, the overall robustness and reliability of the PETRIC has not been thoroughly explored in imaging systems operating daily under a variety of conditions. Future PETRIC designs will certainly need to provide as much durability as possible to keep repairs and hardware replacement to a minimum.

The low-noise photodiode arrays have proven able to fulfill their role in this 
compact gamma camera technology and hence further development work is not immediately necessary. Improved packaging to make the photodiode arrays more robust and therefore easier to test and incorporate into module assemblies would be a major benefit, however. Further reductions in leakage current would also be a boon, but the imaging improvement would be small relative to the research effort required. A final reason for further developing the low-noise photodiode arrays would simply be to implement them "as is" in smaller pixel sizes, since future cameras may potentially use slightly smaller pixels to modestly improve intrinsic spatial resolution and offer a lesser input load to the readout IC channels. A completely different approach for future compact gamma cameras may even involve replacing the photodiodes altogether with extremely compact PMTs that are currently under development (Hamamatsu Photonics K.K., Hamamatsu City, Japan), but these have yet to be proven on their own, let alone in a camera.

The Monte Carlo scintimammography simulation program offers a number of possible avenues for improvement. Currently absent in the code are the implementation of both collimator septal penetration and $\mathrm{CsI}(\mathrm{Tl})$ (or CdZnTe) crystal penetration. With the latter a gamma ray may be absorbed in a crystal adjacent to the "correct" one or, in the case of Compton scatter, it may deposit its energy in two separate crystals. Also of value would be a more complex and realistic phantom patient and a wider variety of camera viewing angles. A more advanced Monte Carlo code incorporating these improvements would be useful in evaluating the expected performance of the various compact, discrete gamma camera designs that may be considered in the future. Of particular interest once again will be collimator design, pixel size, viewing angle, the roles of background uptake and Compton scattering, and the impact of energy resolution under various conditions. For example, while in the future square hole tungsten laminate collimators will likely be cheaper and may therefore see greater use in discrete imaging devices, it would be interesting to study how the performance gains they offer are affected when septal penetration is included in the analysis. 
Another avenue for future development of the $\mathrm{CsI}(\mathrm{Tl}) /$ photodiode technology would be its application to imaging problems other than scintimammography. Because the camera design is both compact and modular, it offers a great deal of flexibility both for the camera area/size/configuration and for the views it can achieve. This potentially makes it an excellent choice for single photon imaging of small organs (e.g., the prostate), for nuclear cardiology, or for small surgical probes. It could also prove valuable in the role of small animal imaging, which is an area of active research for PET instrumentation [142, 143, 144]. Finally, despite the fact that planar scintimammography has generally proven equally or more useful than breast SPECT, there has been contemporary interest in further exploring tomographic approaches $[145,146]$. The advantages offered by compact gamma cameras may be of benefit here and are worth exploring.

In summary, $\mathrm{CsI}(\mathrm{Tl})$ scintillator/Si PIN photodiode detectors with custom IC readout offer promising prospects for compact gamma cameras. With further development and study such imaging devices may prove useful in clinical scintimammography. 


\section{Appendix A}

\section{Publications}

Portions of this thesis project are presented in the following publications:

[A-1] G.J. Gruber, W.S. Choong, W.W. Moses, S.E. Derenzo, S.E. Holland, M. Pedrali-Noy, B. Krieger, E. Mandelli, G. Meddeler, and N.W. Wang, "A compact 64-pixel $\mathrm{CsI}(\mathrm{Tl}) / \mathrm{Si}$ PIN photodiode imaging module with IC readout," IEEE Transactions on Nuclear Science, submitted for publication, 2000.

[A-2] M. Pedrali-Noy, G.J. Gruber, B. Krieger, E. Mandelli, G. Meddeler, W.W. Moses, and V. Rosso, "PETRIC-A Positron Emission Tomography Readout Integrated Circuit," IEEE Transactions on Nuclear Science, submitted for publication, 2000.

[A-3] G.J. Gruber, W.W. Moses, and S.E. Derenzo, "Monte Carlo simulation of breast tumor imaging properties with compact, discrete gamma cameras," IEEE Transactions on Nuclear Science, vol. 46, pp. 2119-2123, 1999.

[A-4] G.J. Gruber, W.W. Moses, S.E. Derenzo, N.W. Wang, E. Beuville, and M.H. Ho, "A discrete scintillation camera module using silicon photodi- 
ode readout of $\mathrm{CsI}(\mathrm{Tl})$ crystals for breast cancer imaging," IEEE Transactions on Nuclear Science, vol. 45, pp. 1063-1068, 1998.

[A-5] N.W. Wang, G. Conte, S.E. Holland, N.P. Palaio, G.J. Gruber, and W.W. Moses, "Improved photosensivite contact for back-illuminated silicon photodiode arrays," IEEE Nuclear Science Symposium and Medical Imaging Conference Abstract Book, p. 12, 1998.

[A-6] W.W. Moses, S.E. Derenzo, G.J. Gruber, R.H. Huesman, and T.F. Budinger, "Potential for SPECT cameras utilizing photodiode readout of scintillator crystals," Journal of Nuclear Medicine, vol. 38, 31P, 1997.

[A-7] S.E. Holland, N.W. Wang, and W.W. Moses, "Development of low noise, back-side illuminated silicon photodiode arrays," IEEE Transactions on Nuclear Science, vol. 44, pp. 443-447, 1997.

[A-8] W.W. Moses, E. Beuville, and M.H. Ho, "A 'Winner-Take-All' IC for determining the crystal of interaction in PET detectors," IEEE Transactions on Nuclear Science, vol. 43, pp. 1615-1618, 1996.

[A-9] W.W. Moses, I. Kipnis, and M.H. Ho, "A 16-channel charge sensitive amplifier IC for a PIN photodiode array based PET detector module," IEEE Transactions on Nuclear Science, vol. 41, pp. 1469-1472, 1994. 


\section{Bibliography}

[1] J.R. Harris, M.E. Lippman, U. Veronesi, et al., "Breast cancer," New England Journal of Medicine, vol. 327, pp. 319-328, 1992.

[2] S.L. Parker, T. Tong, S. Bolden, et al., "Cancer statistics, 1997," A Cancer Journal for Clinicians, vol. 47, pp. 5-27, 1997.

[3] E.J. Sondik, L.G. Kessler, and L.A.G. Ries, eds., Cancer Statistics Review: 1973-1986, including a report on the status of cancer control, National Cancer Institute, Bethesda, MD, NIH Publ. No. 89-2789, 1989.

[4] B.A. Miller, L.A.G. Ries, B.F. Hankey, et al., eds., Cancer Statistics Review: 1973-1989, National Cancer Institute, Bethesda, MD, NIH Publ. No. 92-2789, 1992.

[5] E.J. Feuer, L.M. Wun, C.C. Boring, et al., "The lifetime risk of developing breast cancer," Journal of the National Cancer Institute, vol. 85, pp. 892-897, 1993.

[6] J.L. Kelsey and M.D. Gammon, "The epidemiology of breast cancer," Cancer, vol. 41, pp. 146-165, 1991.

[7] M. Moskowitz and P.S. Gartside, "Evidence of breast cancer mortality reduction: aggressive screening in women under age 30," American Journal of Roentgenology, vol. 138, pp. 911-916, 1982. 
[8] J.D.F. Habbema, G.V.v. Oortmarssen, D.J.v. Putten, et al., "Age-specific reduction in breast cancer mortality by screen: an analysis of the results of Health Insurance Plan of greater New York study," Journal of the National Cancer Institute, vol. 77, pp. 317-320, 1986.

[9] S.H. Heywang-Köbrunner, I. Schreer, and D.D. Dershaw, Diagnostic Breast Imaging, Thieme, New York, 1997.

[10] R. Taillefer, I. Khalkhali, A.D. Waxman, et al., eds., Radionuclide Imaging of the Breast, Marcel Dekker, New York, 1998.

[11] Nuclear Fields, Inc., Des Plaines, IL, product notes, 1999.

[12] H.O. Anger, "Survey of radioisotope cameras," Instrument Society of America, vol. 5, pp. 311-344, 1966.

[13] J.A. Sorenson and M.E. Phelps, Physics in Nuclear Medicine, Second Edition, Grune \& Stratton, Inc., Orlando, 1987.

[14] T.F. Budinger, "Physical attributes of single-photon tomography," Journal of Nuclear Medicine, vol. 21, pp. 579-592, 1980.

[15] "Strategies for managing the breast cancer research program," National Academy of Sciences Institute of Medicine Report to the U.S. Army Medical Research and Development Command, 1993.

[16] "American Cancer Society Breast Cancer Resource Center," http://www2.cancer.org/bcn/information.html, 2000.

[17] D. Reintgen, S.E. Singletary, S. Klimberg, et al., eds., "BreastDoctor.com," http://www.breastdoctor.com/index.htm, 1998.

[18] L. Tabar, G. Fagerberg, N.E. Day, et al., "Breast cancer treatment and natural history: new insights from results of screening," The Lancet, vol. 339, pp. 412, 1992. 
[19] W.D. Dupont, D.L. Page, F.F. Parl, et al., "Long-term risk of breast cancer in women with fibroadenoma," New England Journal of Medicine, vol. 331, pp. 10-15, 1994.

[20] M. Friedrich, "Technik und ergebnisse der mammographie," Radiologe, vol. 33, pp. 243, 1993.

[21] S. Shapiro, W. Venet, and P. Strax, "Ten- to fourteen-year effect of screening on breast cancer mortality," Journal of the National Cancer Institute, vol. 69, pp. 349-355, 1982.

[22] I. Khalkhali, I. Mena, E. Jouanne, et al., "Prone scintimammography in patients with suspicion of carcinoma of the breast," Journal of the American College of Surgeons, vol. 178, pp. 491-497, 1994.

[23] E.L. Thurfjell, L.H. Holmberg, and I.R. Persson, "Screening mammography: sensitivity and specificity in realtion to hormone replacement therapy," Radiology, vol. 203, pp. 339-341, 1997.

[24] S.A. Feig, G.A. Shaber, and A. Patchefskly, "Analysis of clinically occult and mammographically occult breast tumors," American Journal of Roentgenology, vol. 128, pp. 403-408, 1977.

[25] D.B. Kopans, "Positive predictive value of mammography," American Journal of Roentgenology, vol. 158, pp. 521-526, 1992.

[26] R.E. Bird, T.W. Wallace, and B.C. Yankaskas, "Analysis of cancers missed at the screening mammography," Radiology, vol. 184, pp. 613-617, 1992.

[27] B.D. Mann, A.E. Giuliano, L.W. Bassett, et al., "Delayed diagnosis of breast cancer as a result of normal mammograms," Archives of Surgery, vol. 118 pp. $23-25,1983$. 
[28] L. Kalisher, "Factors influencing false negative rates in mammography," Radiology, vol. 133, pp. 297-301, 1979.

[29] D.B. Kopans, "Conventional wisdom: observation, experience, anecdote and science in breast imaging," American Journal of Roentgenology, vol. 162, pp. 299-303, 1994.

[30] E.C. Coveney, J.G. Geraghty, R. O'Laoide, et al., "Reasons underlying negative mammography in patients with palpable breast cancer," Clinical Radiology, vol. 49, pp. 123-125, 1994.

[31] B. Fisher, M. Bauer, L. Widkerham, et al., "Relations of number of positive axillary nodes to the prognosis of patients with primary breast cancer," Cancer, vol. 52, pp. 1551-1557, 1983.

[32] P.J. Morris and R.A. Malt, In Oxford textbook of surgery, pp. 815, Oxford Medical Publications, Oxford, 1994.

[33] W.W.M. Lam, W.T. Yang, Y.L. Chan, et al., "Detection of axillary lymph node metastases in breast carcinoma by Tc-99m-sestamibi breast scintigraphy, ultrasound, and conventional mammography," European Journal of Nuclear Medicine, vol. 23, pp. 498-503, 1996.

[34] S. Larson, M.D., Sloan Kettering, personal communication.

[35] V.W. Lee, E.J. Sax, D.B. McAneny, et al., "A complementary role for thallium201 scintigraphy with mammography in the diagnosis of breast cancer," Journal of Nuclear Medicine, vol. 34,pp. 2095-2100, 1993.

[36] A.M. Sehweil, J.H. McKillop, R. Milroy, et al., "201 Tl scintigraphy in the staging of lung cancer, breast cancer and lymphoma," Nuclear Medicine Communications, vol. 11, pp. 263-269, 1990. 
[37] A. Waxman, N. Nagaraj, and G. Ashok, "Sensitivity and specificity of Tc-99m methoxy isobutal isonitrile (MIBI) in the evaluation of primary carcinoma of the breast: comparison of palpable and nonpalpable lesions with mammography," Journal of Nuclear Medicine, vol. 35, pp. 22P, 1994.

[38] I. Khalkhali, I. Mena, and L. Diggles, "Review of imaging techniques for the diagnosis of breast cancer: a new role of prone scintimammography using technetium-99m sestamibi," European Journal of Nuclear Medicine, vol. 21, pp. 357-362, 1994.

[39] I. Khalkhali, J. Cutrone, I. Mena, et al., "Clinical and pathologic follow-up of 100 patients with breast lesions studied with scintimammography," Journal of Nuclear Medicine, vol. 35, pp. 22P, 1994.

[40] S. Lastoria, P. Varrella, and C. Mainolfi, "Technetium-99m-sestamibi scintigraphy in the dagnosis of primary breast cancer," Journal of Nuclear Medicine, vol. 35, pp. 22P, 1994.

[41] J. Villanueva-Meyer, M.H. L. Leonard Jr., S. Ali, et al., "Technetium-99msestamibi in the evaluation of mammographic abnormalities," Journal of Nuclear Medicine, vol. 35 pp. 229P, 1994.

[42] J. Villanueva-Meyer, M.H.L. Leonard Jr., E. Briscoe, et al., "Mammoscintigraphy with technetium-99m-sestamibi in suspected breast cancer," Journal of Nuclear Medicine vol. 36, pp. 926-930, 1996.

[43] L.I. Elmon-Noingeon, D. Piwnica-Worms, A.D.V.d. Abbeele, et al., "Uptake of the cation hexakis(2-methoxyisobutyl-isonitrile)-technetium-99m by human carcinoma cell lines in vitro," Cancer Research, vol. 50, pp. 2198-2202, 1990.

[44] D. Piwnica-Worms, M.L. Chiu, M. Budding, et al., "Functional imaging of multidrug-resistant P-glycoprotein with an organotechnetium complex," Cancer Research, vol. 53, pp. 977-984, 1993. 
[45] D. Piwnica-Worms, J.F. Kronauge, and M.L. Chiu, "Uptake and retention of hexakis (2-methoxyisobutyl isonitrile) technetium(I) in cultured chick myocardial cells," Circulation, vol. 82, pp. 1826-1838, 1990.

[46] D. Piwnica-Worms, J.F. Kronauge, and M.L. Chiu, "Enhancement by tetraphenylborate of technetium-99m-MIBI uptake kinetics and accumulation in cultured chick myocardial cells," Journal of Nuclear Medicine, vol. 32, pp. 1992-1999, 1991.

[47] P.A. Carvalho, M.L. Chiu, J.F. Kronaug, et al., "Subcellular distribution and analysis of Tc-99m MIBI in isolated perfused rat hearts," Journal of Nuclear Medicine, vol. 33, pp. 1516-1521, 1992.

[48] I. Khalkhali, J. Cutrone, I. Mena, et al., "Technetium-99m-sestamibi scintimammography of breast lesions: clinical and pathological follow-up," Journal of Nuclear Medicine, vol. 36, pp. 1784-1789, 1996.

[49] J. Maublant, M.D. Latour, D. Mestas, et al., "Technetium-99m-sestamibi uptake in breast tumor and associated lymph nodes," Journal of Nuclear Medicine, vol. 37, pp. 922-925, 1996.

[50] F. Scopinaro and O. Schillaci, "The use of technetium-99m-sestamibi to indicate breast cancer invasiveness," European Journal of Nuclear Medicine, vol. 22, pp. 582-585, 1995.

[51] C.H.J. van Eijk, E.P. Krenning, A. Bootsma, et al., "Somatostatin receptor scintigraphy of primary breast cancer and its predictive value in the follow-up in comparison with CEA and CA 15-3," Journal of Nuclear Medicine, vol. 35, pp. 21P, 1994.

[52] S. Piccolo, S. Lastoria, C. Mainolfi, et al., "Role of Tc-99m MDP scintigraphy in the diagnosis of primary breast cancer," Journal of Nuclear Medicine, vol. 35, pp. 22P, 1994. 
[53] M.R. Vieira and J.H.B. Weinholtz, "Technetium-99m tetrofosmin scintigraphy in the diagnosis of breast cancer," European Journal of Surgical Oncology, vol. 22, pp. 331-334, 1996.

[54] R. Taillefer, A. Robidoux, S. Turpin, et al., "Detection of axillary lymph node involvement with Tc-99m-sestamibi imaging in breast cancer detection," Journal of Nuclear Medicine, vol. 37, pp. 75P, 1996.

[55] I. Khalkhali, J. Villanueva-Meyer, S.L. Edell, et al., "Diagnostic accuracy of Tc$99 \mathrm{~m}$ sestamibi breast imaging in breast cancer detection," Journal of Nuclear Medicine, vol. 36, pp. 718-724, 1995.

[56] S. Piccolo, S. Lastoria, C. Mainolfi, et al., "Technetium-99m-methylene diphosphonate scintimammography to image primary breast cancer," Journal of $\mathrm{Nu}$ clear Medicine, vol. 36, pp. 718-724, 1995.

[57] H. Palmedo, H.H. Biersack, S. Lastoria, et al., "Scintimammography with Tc$99 \mathrm{~m}$ MIBI for breast cancer detection: results of the prospective European multicenter trial," Journal of Nuclear Medicine, vol. 38, pp. 20-21P, 1997.

[58] I. Khalkhali, J. Villanueva-Meyer, S.L. Edell, et al., "Impact of breast density on the diagnostic accuracy of Tc-99m sestamibi breast imaging in the detection of breast cancer," Journal of Nuclear Medicine, vol. 37, pp.74-74P, 1996.

[59] T.H. Helbich, A. Becherer, S. Trattnig, et al., "Differentiation of benign and malignant breast lesions: MR imaging versus Tc-99m sestamibi scintimammography," Radiology, vol. 202, pp.421-429, 1997.

[60] H. Palmedo, A. Schomburg, F. Grunwald, et al., "Technetium-99mscintimammography for suspicious breast lesions," Journal of Nuclear Medicine, vol. 37, pp. 626-630, 1996. 
[61] F. Scopinaro, O. Schillaci, W. Ussof, et al., "A three center study on the diagnostic accuracy of ${ }^{99 m}$ Tc-MIBI scintimammography," Anticancer Research, vol. 17, pp. 1631-1634, 1997.

[62] C.H. Kao, S.J. Wang, and S.H. Yeh, "Tc-99m MIBI uptake in breast carcinoma and axillary lymph node metastases," Clinical Nuclear Medicine, vol. 19, pp. 898-900, 1994.

[63] B. Fisher, M. Bauer, D.L. Wickerham, et al., "Relation of number of positive axillary nodes to the prognosis of patients with primary breast cancer," Cancer, vol. 52, pp. 1551-1557, 1983.

[64] L.P. Adler, P.F. Faulhaber, K.C. Schnur, et al., "Axillary lymph node metastases: screening with [F-18]2-deoxy-2- fluoro-D-glucose (FDG) PET," Radiology, vol. 203, pp. 323-327, 1997.

[65] M. Jacobs, J. Mantil, C. Peterson, et al., "FDG PET in breast cancer," Journal of Nuclear Medicine, vol. 35, pp. 142P, 1994.

[66] C.J. Thompson, K. Murthy, I.N. Weinberg, et al., "Feasibility study for positron emission mammography," Medical Physics, vol. 21, pp. 529-538, 1994.

[67] R.L. Wahl, R.L. Cody, G.D. Hutchins, et al., "Primary and metatatic breast carcinoma: initial clinical evaluation with PET with the radiolabeled glucose analog 2-[F-18]Fluoro-2-deoxy-D-glucose," Radiology, vol. 179, pp. 765-770, 1991.

[68] W.W. Moses, T.F. Budinger, R.H. Huesman, et al., "PET camera designs for imaging breast cancer and axillary node involvement," Journal of Nuclear Medicine, vol. 36, pp. 69P, 1995.

[69] C.J. Thompson, K. Murthy, R.L. Clancy, et al., "Imaging performance of a PEM-I: A high resolution system for positron emission mammography," IEEE Nuclear Science Symposium Conference Record, vol. 2, pp. 1074-1078, 1995. 
[70] H. Madjar, U. Makowiec, A. Mundinger, et al., "Value of high resolution sonography in breast cancer screening," Ultraschall in der Medizin, vol. 15, pp. 20-23, 1994.

[71] D.B. Kopans, "What is a useful adjunct to mammography?" Radiology, vol. 161, pp. 560-561, 1986.

[72] D.O. Cosgrove, R.P. Kedar, J.C. Bamber, et al., "Breast diseases: colour Doppler US in differential diagnosis," Radiology, vol. 189, pp. 99-104, 1993.

[73] C.I. Perre, V.C.M. Koot, P.d. Hooge, et al., "Colour Doppler ultrasonography in the diagnosis of axillary lymph node metastases in breast cancer," The Breast, vol. 5, pp. 10-12, 1996.

[74] R.P. Kedar, D. Cosgrove, V.R. McCready, et al., "Microbubble constrast agent for color Doppler US - effect on breast masses," Radiology, vol. 198, pp. 679-686, 1996.

[75] W.T. Yang, J. Chang, and C. Metreweli, "Patients with breast cancer: Differences in color Doppler flow and gray-scale US features of benign and malignant axillary lymph nodes," Radiology, vol. 215, pp. 568-573, 2000.

[76] K. Nquyen, A. Waxman, P. Gupta, et al., "Comparison of Tc-99m methoxyisobutylisonitrile (MIBI) and MRI in breast malignancy: the significance of concordant and discordant findings," Journal of Nuclear Medicine, vol. 37, pp. 75P, 1996.

[77] R. Tiling, H. sommer, M. Pechmann, et al., "Semiquantitative scintimammography with sestamibi or contrast enhanced MRI for further evaluation of indeterminate mammograms," Journal of Nuclear Medicine, vol. 37, pp. 156P, 1996.

[78] S.H. Heywang, A. Wolf, E. Pruss, et al., "MR imaging of the breast with GdDTPA: Use and limitations," Radiology, vol. 171, pp. 95-103, 1989. 
[79] S.G. Orel, M.D. Schnall, V.A. Livolsi, et al., "MR imaging with radiologicpathologic correlation," Radiology, vol. 190, pp. 484-493, 1994.

[80] S.E. Harms, D.P. Flamig, K.L. Hesley, et al., "MR imaging of the breast with rotating delivery of excitation off resonance: Clinical experience with pathologic correlation," Radiology, vol. 187, pp. 493-501, 1993.

[81] S.H. Heywang-Köbrunner, Contrast-enhanced MRI of the breast, 2nd ed., Springer, Berlin, 1996.

[82] Thomas Budinger, M.D., Ph.D., Lawrence Berkeley National Laboratory, personal communication.

[83] S. Mussurakis, D.L. Buckley, and A. Horsman, "Prediction of axillary lymph node status in invasive breast cancer with dynamic contrast-enhanced MR imaging," Radiology, vol. 203, pp. 317-321, 1997.

[84] H.O. Anger, "Radioisotope cameras," in G.J. Hine, ed., Instrumentation in Nuclear Medicine, vol. 1, ch. 19, New York, Academic Press, 1967.

[85] NEMA Standards Publication NU 1-1994: Performance Measurements of Scintillation Cameras, National Electrical Manufacturers Association, Washington, DC, 1994.

[86] S.E. Holland, N.W. Wang, and W.W. Moses, "Development of low noise, backside illuminated silicon photodiode arrays," IEEE Transactions on Nuclear Science, vol. 44, pp. 443-447, 1997.

[87] B.S. Avset, J. Ellison, L. Evensen, et al., "Silicon Drift Photodiodes," Nuclear Instruments 85 Methods in Physics Research, vol. A-288, pp. 131-136, 1990.

[88] E. Gramsch, E. Gullikson, W.W. Moses, et al., "Operating characteristics of avalanche photodiodes for PET systems," 1996 IEEE Nuclear Science Symposium Conference Record, vol. 2, pp. 768-772, 1996. 
[89] J. Iwanczyk, N. Dorri, M. Wang, et al., "20-Element $\mathrm{HgI}_{2}$ energy dispersive x-ray array detector system," IEEE Transactions on Nuclear Science, vol. 39, pp. 1275-1280, 1992.

[90] F. Olschner, K. Shah, J. Lund, et al., "Thallium bromide semiconductor xray and gamma-ray detectors," Nuclear Instruments $\&$ Methods in Physics Research, vol. A-322, pp. 504-508, 1992.

[91] M. Squillante, C. Zhou, J. Zhange, et al., "InI nuclear radiation detectors," IEEE Transactions on Nuclear Science, vol. 40, pp. 364-366, 1993.

[92] J. Strobel, N.H. Clinthorne, and W.L. Rogers, "Design studies for a cesium iodide silicon photodiode gamma camera," Journal of Nuclear Medicine, vol. 38, pp. 31P, 1997.

[93] G.J. Gruber, W.W. Moses, S.E. Derenzo, et al., "A discrete scintillation camera module using silicon photodiode readout of $\mathrm{CsI}(\mathrm{Tl})$ crystals for breast cancer imaging," IEEE Transactions on Nuclear Science, vol. 45, pp. 1063-1068, 1998.

[94] B.E. Patt, J.S. Iwanczyk, C. Rossington Tull, et al., "High resolution CsI(Tl)/SiPIN detector development for breast imaging," IEEE Transactions on Nuclear Science, vol. 45, pp. 2126-2131, 1998.

[95] G.J. Gruber, W.W. Moses, and S.E. Derenzo, "Monte Carlo Simulation of Breast Tumor Imaging Properties with Compact, Discrete Gamma Cameras," IEEE Transactions on Nuclear Science, vol. 46, pp. 2119-2123, 1999.

[96] M.P. Tornai, B.E. Patt, J.S. Iwanczyk, et al., "Discrete scintillator coupled mercuric iodide photodetector arrays for breast imaging," IEEE Transactions on Nuclear Science, vol. 44, pp. 1127-1133, 1997.

[97] D. Gunter, K. Mathews II, and C. Ordonez, "The Interaction of Collimator Lattice Periodicity and Detector Pixelation," IEEE Nuclear Science Symposium Conference Record, vol. 3, pp. 1525-1531, 1998. 
[98] H.B. Barber, H.H Barrett, S.J. Balzer, et al., "Gamma-Ray Imaging Using a CdZnTe Pixel Array and an Ultra-High-resolution, Parallel-Hole Collimator," IEEE Nuclear Science Symposium and Medical Imaging Conference Abstract Book, pp. 30P, 1999.

[99] G. Armantrout, S. Swierkowski, J. Sherohman, et al, "What can be expected from high-Z semiconductor detectors?" IEEE Transactions on Nuclear Science, vol. 24, pp. 121-125, 1977.

[100] L. Kaufman, J. Ewins, W. Rowan, et al., "Semiconductor gamma cameras in nuclear medicine," IEEE Transactions on Nuclear Science, vol. 27, pp. 10731079, 1980.

[101] H.B. Barber, F.L. Augustine, H.H. Barrett, et al., "Semiconductor arrays with multiplexer readout for gamma-ray imaging - results for a 48x48 Ge array," Nuclear Instruments $\&$ Methods in Physics Research, vol. A-353, pp. 361-365, 1994.

[102] M. Singh and D. Doria, "Single photon imaging with electronic collimation," IEEE Transactions on Nuclear Science, vol. 32, pp. 843-847, 1985.

[103] J.F. Butler, S.J. Friesenhahn, C.L. Lingren, et al., "CZT detector arrays for nuclear medicine imaging," IEEE Nuclear Science Symposium Conference Record, vol. 1, pp. 565-568, 1993.

[104] D.G. Marks, H.B. Barber, H.H. Barrett, et al., "A 48x48 CdZnTe array with multiplexer readout," IEEE Nuclear Science Symposium and Medical Imaging Conference Record," vol. 2, pp. 752-756, 1995.

[105] J.F. Butler, C.L. Lingren, S.J. Friesenhahn, et al., "CdZnTe solid-state gamma camera," IEEE Transactions on Nuclear Science, vol. 45, pp. 359-363, 1998.

[106] M. Singh and E. Mumcuoglu, "Design of a CZT based BreastSPECT system," IEEE Transactions on Nuclear Science, vol. 45, pp. 1158-1165, 1998. 
[107] W.L. Ashburn, "Radionuclide imaging of the breast using a solid-state gamma camera," in R. Taillefer, I. Khalkhali, A.D. Waxman, et al., eds., Radionuclide Imaging of the Breast, Marcel Dekker, New York, 1998.

[108] H. Kume, S. Suzuki, J. Takeuchi, et al., "Newly developed photomultiplier tubes with position sensitivity capability," IEEE Transactions on Nuclear Science, vol. 32, pp. 448-452, 1985.

[109] T. Hayashi, "New photomultiplier tubes for medical imaging," IEEE Transactions on Nuclear Science, vol. 36, pp. 1078-1083, 1989.

[110] J.G. Rogers, "Testing an improved scintillation camera for PET and SPECT," IEEE Transactions on Nuclear Science, vol. 33, pp. 519-522, 1986.

[111] K. Blazek, F. de Notaristefani, F. Maly, et al., "YAP multi-crystal gamma camera prototype," IEEE Transactions on Nuclear Science, vol. 42, pp. 1474$1482,1995$.

[112] F. de Notaristefani, R. Pani, F. Scopinaro, et al., "First results from a YAP:Ce gamma camera for small animal studies," IEEE Transactions on Nuclear Science, vol. 43, pp. 3264-3271, 1996.

[113] N.J. Yasillo, R.A. Mintzer, J.N. Aarsvold, et al., "A single-tube miniature gamma camera," IEEE Nuclear Science Symposium Conference Record, vol. 1, pp. 1073-1076, 1993.

[114] F. Vittori, T. Malatesta, and F. de Notaristefani, "The YAP camera: and accurate gamma camera particularly suitable for new radiopharmaceuticals research," IEEE Transactions on Nuclear Science, vol. 44, pp. 47-53, 1997.

[115] C.S. Levin, E.J. Hoffman, M.P. Tornai, et al., "PSPMT and PIN diode designs of a small scintillation camera for imaging malignant breast tumors," IEEE Nuclear Science Symposium Conference Record, vol. 2, pp. 1196-1200, 1996. 
[116] R. Pani, G. De Vincentis, F. Scopinaro, et al., "Dedicated gamma camera for single photon emission mammography (SPEM)," IEEE Transactions on Nuclear Science, vol. 45, pp. 3127-33., 1998.

[117] D. Steinbach, S. Cherry, N. Doshi, et al., "A small scintimammography detector based on a 5" PSPMT and a crystal scintillator array," IEEE Nuclear Science Symposium Conference Record, vol. 2, pp. 1255-1259, 1997.

[118] F.S. Goulding, "Pulse-shaping in low-noise nuclear amplifiers: a physical approach to noise analysis," Nuclear Instruments and Methods, vol. 100, pp. 493$504,1972$.

[119] W.W. Moses, I. Kipnis, and M.H. Ho, "A 16-channel charge sensitive amplifier IC for a PIN photodiode arrays based PET detector module," IEEE Transactions on Nuclear Science, vol. 41, pp. 1469-1472, 1994.

[120] W.W. Moses, E. Beuville, and M.H. Ho, "A 'Winner- Take-All' IC for determining the crystal of interaction in PET detectors," IEEE Transactions on Nuclear Science, vol. 43, pp. 1615-1618, 1996.

[121] S. Holland, "Fabrication of detectors and transistors on high-resistivity silicon," Nuclear Instruments 8 Methods in Physics Research, vol. A-275, pp. 537-541, 1989.

[122] M. Moszynski, M. Kapusta, M. Mayhugh, et al., "Absolute light output of scintillators," IEEE Transactions on Nuclear Science, vol. 44, pp. 1051-1061, 1997.

[123] William Moses, Ph.D., Lawrence Berkeley National Laboratory, personal communication.

[124] Bicron, Washougal, WA, product note SC-118, 1997. 
[125] M. Ljungberg, S.E. Strang, J.E. Englund, et al., "Monte Carlo simulation of high count rate scintillation camera imaging," IEEE Nuclear Science Symposium Conference Record, vol. 4, pp. 1682-1686, 1994.

[126] J.S. Fleming and D.E. Simpson, "A technique for simulation of the point spread function of a gamma camera," Physics in Medicine and Biology, vol. 39, pp. 1457-1473, 1994.

[127] J.S. Fleming, "Evaluation of a technique for simulation of gamma camera images," Physics in Medicine and Biology, vol. 41, pp. 1855-1861, 1996.

[128] M. Spisar, J.N. Aarsvold, R.A. Mintzer, "DETECT97 simulation studies of light output in a full field-of-view small gamma camera," IEEE Nuclear Science Symposium Conference Record, vol. 2, pp. 1188-1192, 1997.

[129] D. Steinbach, A.R. Goode, F. Farzanpay, et al., "Development of a small-fieldof-view scintimammography camera: measurements and simulations," Proceedings of the SPIE - The International Society for Optical Engineering, vol. 3115, pp. 10-20, 1997.

[130] W.H. Press, S.A. Teukolsky, W.T. Vetterling, et al., Numerical Recipes in C, Second Edition, Cambridge University Press, Cambridge, 1993.

[131] P.R.G. Virador, "Image Reconstruction for a Positron Emission Tomograph Optimized for Breast Cancer Imaging," Ph.D. thesis, Univserity of California at Berkeley, 2000.

[132] Iraj Khalkhali, M.D., UCLA Medical Center, personal communication.

[133] A.E. Rose, Vision: Human and Electronic, Plenum Press, New York, 1973.

[134] R. Pani, F. Scopinaro, R. Pellegrini, et al., "The role of Compton background and breast compression on cancer detection in scintimammography," Anticancer Research, vol. 17, pp. 1645-1649, 1997. 
[135] M. Pedrali-Noy, W.W. Moses, E. Mandelli, et al., "PETRIC-A Positron Emission Tomography Readout Integrated Circuit," IEEE Nuclear Science Symposium and Medical Imaging Conference Abstract Book, in press, 2000.

[136] G. Gramegna, P.P. P'Connor, P. Rehak, et al., "CMOS preamplifier for lowcapacitance detectors," Nuclear Instruments 8 Methods in Physics Research, vol. A-390, pp. 241-250, 1997.

[137] P.R. Gray and R.G. Meyer, Analysis and Design of Analog Integrated Circuits, Third Edition, John Wiley \& Sons, Inc., New York, 1993.

[138] B. Krieger, I. Kipnis, and B.A. Ludewigt, "XPS: A Multi-Channel PreamplifierShaper IC for X-Ray Spectroscopy," IEEE Transactions on Nuclear Science,, vol. 45, pp. 732-734, 1998.

[139] R. Freifelder, J.S. Karp, M. Geagan, et al., "Design and performance of the HEAD PENN-PET scanner," IEEE Transactions on Nuclear Science, vol. 41, pp. 1436-1440, 1994.

[140] D.A. Mankoff, G. Muehllehner, G.E. Miles, "A local coincidence triggering system for PET tomographs composed of large-area position-sensitive detectors," IEEE Transactions on Nuclear Science, vol. 37, pp. 730-736, 1990.

[141] N.W. Wang, G. Conte, S.E. Holland, et al., "Improved photosensivite contact for back-illuminated silicon photodiode arrays," IEEE Nuclear Science Symposium and Medical Imaging Conference Abstract Book, p. 12, 1998.

[142] A. Chatziioannou, R.W. Silverman, K. Meadors, et al., "Techniques to improve the spatial sampling of microPET - a high resolution animal PET tomograph," IEEE Transactions on Nuclear Science, vol. 47, pp. 422-427, 2000.

[143] S. Siegel, J.J. Vaquero, L. Aloj, et al., "Initial results from a PET planar small animal imaging system," IEEE Transactions on Nuclear Science, vol. 46, pp. 571-575, 1999. 
[144] J.A. Correia, C.A. Burnham, D. Kaufman, et al., "Development of a small animal PET imaging device with resolution approaching $1 \mathrm{~mm}$," IEEE Transactions on Nuclear Science, vol. 46, pp. 631-635, 1999.

[145] K. Yutani, E. Shiba, H. Kusuoka, et al., "Comparison of FDG-PET with MIBISPECT in the detection of breast cancer and axillary lymph node metastasis," Journal of Computer Assisted Tomography, vol. 24, pp. 274-280, 2000.

[146] R. Obwegeser, P. Berghammer, M. Rodrigues, et al., "Use of Tc-99m-furifosmin scintigraphy - planar and SPECT - to evaluate suggestive palpable and nonpalpable breast lesions," Journal of Nuclear Medicine, vol. 41, pp. 426-428, 2000. 